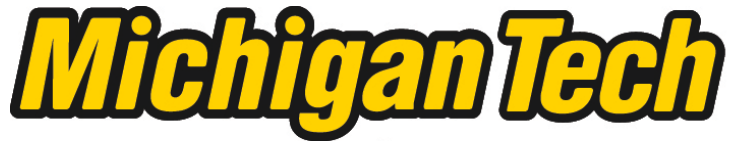 \\ Michigan Technological University Create the Future Digital Commons @ Michigan Tech
}

Dissertations, Master's Theses and Master's Reports - Open

Dissertations, Master's Theses and Master's

Reports

2010

\section{Structural and surface property characterization of titanium dioxide nanotubes for orthopedic implants}

Tolou Shokuhfar

Michigan Technological University

Follow this and additional works at: https://digitalcommons.mtu.edu/etds

Part of the Mechanical Engineering Commons

Copyright 2010 Tolou Shokuhfar

\section{Recommended Citation}

Shokuhfar, Tolou, "Structural and surface property characterization of titanium dioxide nanotubes for orthopedic implants", Dissertation, Michigan Technological University, 2010.

https://doi.org/10.37099/mtu.dc.etds/407

Follow this and additional works at: https://digitalcommons.mtu.edu/etds

Part of the Mechanical Engineering Commons 


\title{
STRUCTURAL AND SURFACE PROPERTY \\ CHARACTERIZATION OF TITANIUM DIOXIDE \\ NANOTUBES FOR ORTHOPEDIC IMPLANTS
}

By

Tolou Shokuhfar

\begin{abstract}
A DISSERTATION
Submitted in partial fulfillment of the requirements for the degree of DOCTOR OF PHILOSOPHY
\end{abstract}

(Mechanical Engineering-Engineering Mechanics)

\section{MICHIGAN TECHNOLOGICAL UNIVERSITY}

2010

(C) 2010 Tolou Shokuhfar 
This dissertation, "Structural and Surface Property Characterization of Titanium Dioxide Nanotubes for Orthopedic Implants" is hereby approved in partial fulfillment of the requirements for the Degree of DOCTOR OF PHILOSOPHY IN MECHANICAL ENGINEERING-ENGINEERING MECHANICS.

Department of Mechanical Engineering-Engineering Mechanics

\section{Signatures:}

Dissertation Advisor:

Prof. Craig Friedrich

Committee Members:

Prof. Gregory M. Odegard

Prof. Chang K. Choi

Prof. Patricia A. Heiden

Department Chair:

Prof. William W. Predebon

Date: $12 / 13 / 2010$ 


\begin{abstract}
This research focused on the to modification of the surface structure of titanium implants with nanostructured morphology of $\mathrm{TiO}_{2}$ nanotubes and studied the interaction of nanotubes with osteoblast cells to understand the parameters that affect the cell growth. The electrical, mechanical, and structural properties of $\mathrm{TiO}_{2}$ nanotubes were characterized to establish a better understanding on the properties of such nanoscale morphological structures.

To achieve the objectives of this research work I transformed the titanium and its alloys, either in bulk sheet form, bulk machined form, or thin film deposited on another substrate into a surface of titania nanotubes using a low cost and environmentally friendly process. The process requires only a simple electrolyte, low cost electrode, and a DC power supply. With this simple approach of scalable nanofabrication, a typical result is nanotubes that are each approximately $100 \mathrm{~nm}$ in diameter and have a wall thickness of about 20nm. By changing the fabrication parameters, independent nanotubes can be fabricated with open volume between them. Titanium in this form is termed onedimensional since electron transport is narrowly confined along the length of the nanotube. My Ph.D. accomplishments have successfully shown that osteoblast cells, the cells that are the precursors to bone, have a strong tendency to attach to the inside and outside of the titanium nanotubes onto which they are grown using their filopodia - cell's foot used for locomotion - anchored to titanium nanotubes. In fact it was shown that the cell prefers to find many anchoring sites. These sites are critical for cell locomotion during the first several weeks of maturity and upon calcification as a strongly anchored bone cell. In addition I have shown that such a surface has a greater cell density than a smooth titanium surface. My work also developed a process that uses a focused and controllably rastered ion beam as a nano-scalpel to cut away sections of the osteoblast cells to probe the attachment beneath the main cell body. Ultimately the more rapid growth of osteoblasts, coupled with a stronger cell-surface interface, could provide cost reduction, shorter rehabilitation, and fewer follow-on surgeries due to implant loosening.
\end{abstract}




\section{ACKNOWLEDGEMENT}

This research project would not have been possible without the support of many people. I wish to express my gratitude to my supervisor, Prof. Dr. Craig Friedrich who was abundantly helpful and offered invaluable assistance, support and guidance. I am heartily thankful for his encouragement, guidance and support from the initial to the final level which, enabled me to develop an understanding of the science and nature of my $\mathrm{PhD}$ work. His perpetual energy and enthusiasm in research had motivated all his advisees, including me. In addition, he was always accessible and willing to help his students with their research. As a result, research life became smooth and rewarding for me. Besides, he sets an example of a world-class researcher for his rigor and passion on research.

Deepest gratitude also to the members of the supervisory committee, Prof. Dr. Chang K.Choi, Prof. Dr. Gregory Odegard, and Prof. Dr. Patritia Heiden, without whose knowledge and assistance this study would not have been successful. It was absolutely a wonderful experience to work with them. Special thanks also to, Prof. Tammy Donahue and all my friends, especially Jen-Yung Chang, Dr. Anjana Ashtana, Dr. Karl Walzak, Qi Gao and Chee Huei Lee for invaluable assistance. I am grateful to come across Martha at work as a life-long friend.

I was delighted to work with Mr. Owen Mills and I am thankful for all his assistance. I would also like to convey thanks to the Ministry and Faculty Of Mechanical Engineering, Materials Science and Engineering, MuSTI for providing the financial means and laboratory facilities.

My deepest gratitude goes to my family for their unflagging love and support throughout my life; this dissertation is simply impossible without them. I wish to express my love and gratitude to them as the dearest and most beloved people in my life whom I am indebted to for their care and love. My husband Prof. Reza Shahbazian Yassar for his constant support and endless love. Whenever I encountered difficulties his warm words would lighten up my dark days and no sleep nights of hard work, like the sparkling sea 
under the sunrise awaiting a warm happy day with golden sands, palm trees and tropical fruits. He always inspires me in both our academic and love lives. My father Prof. Dr. Ali Shokuhfar, who spare no effort to provide the best possible environment for me to grow up and have the highest level of education. He had been a role model for me to follow unconsciously and has always been one of my best counselors. Apparently, he is an angel from God shedding light on secular issues from a spiritual angle. I cannot ask for more from my mother, Dr. Mina Azimi, as she is simply perfect. I have no suitable word that can fully describe her everlasting love to me. I feel proud of my brother Dr. Taha-Amin Shokuhfar for his talents and his extreme kindness and constant source of encouragement during my life. My beloved grandmother, Razyeh Navvab Lahijani who is like my spiritual mother who drawing me close to the Lord and for many sleepless nights with her accompanying me when I was not feeling well. I remember, most of all, her delicious dishes. My dearest in-laws, Shahbazian Yassar's family, who were always available to chat with me and cheer me up and for their understanding \& endless love, through the duration of my studies. I would like to express my feeling toward the endless support of my family with few words: Many thanks for making the moment of $\mathrm{PhD}$ graduation, feel so happy and bright...So refreshing as summer breeze blowing in hair or simply the taste of peppermint chocolate..., I would like to closed my eyes, and imagined myself in a lavender garden with blue purplish sky, singing birds and cherry blossoms surrounded by all of you my dearesrts. Many thanks my dear thoughtful family, for all your warm encouraging support who made my dreams come true.

Lastly, I offer my regards and blessings to all of those who supported me in any respect during the completion of my $\mathrm{PhD}$.

Tolou Shokuhfar 


\section{Table of Contents}

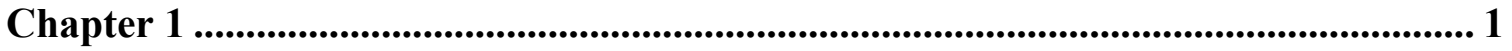

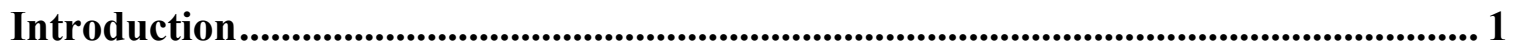

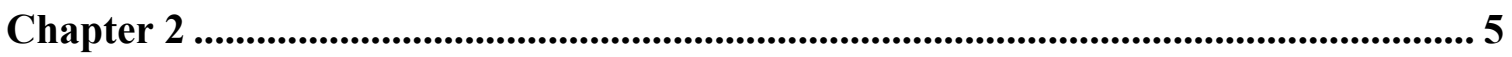

SLE Technique for Surface Engineering of Titanium Rods with Modified Porous Structures............................................................................................................................... 5

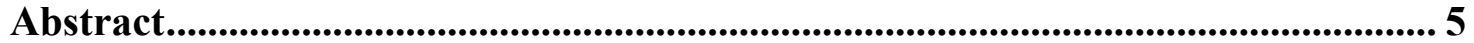

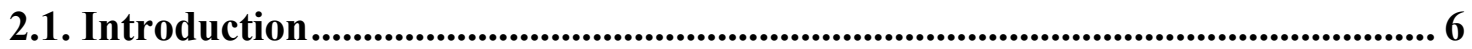

2.2. Experimental Procedure ................................................................................................. 8

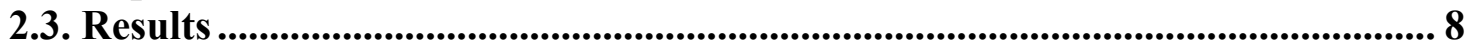

2.3.1 Selective Electrochemical Etching (SEE) Process ………….......................... 8

2.3.2. Sandblasting Followed by Acid etching (SLA) ............................................. 13

2.3.3. Sandblasting Followed by Electro Etching (SLE) Processes ......................... 14

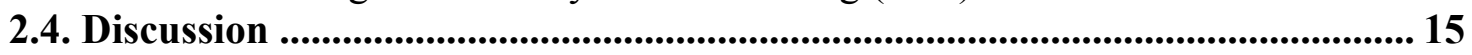

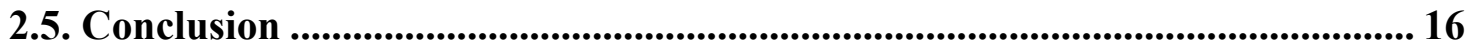

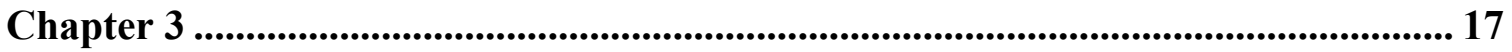

Synthesis of $\mathrm{TiO}_{2}$ Nanotubes....................................................................................... 17

Abstract............................................................................................................................... 17

3.1. Introduction..................................................................................................................... 18

3.2. Mechanism of $\mathrm{TiO}_{2}$ Nanotube Formation .......................................................... 20

3.3. Experimental Procedure ......................................................................................... 21

3.4. Results and Discussion.............................................................................................. 21

3.4.1 Effect of Driving Voltage on Anodization Kinetics ......................................... 21

3.4.2 Effect of Anodization Time on the Diameter of Nanotubes ............................. 23

3.4.3 Effect of Voltage on the Diameter of Nanotubes............................................. 24

3.4.4 Effect of Time on the Length of Nanotubes ................................................... 25

3.4.5 The Effect of Voltage on the Length of Nanotubes........................................ 26

3.5. Chemistry of $\mathrm{TiO}_{2}$ Nanotubes ................................................................................... 28

3.6. Conclusion ...................................................................................................................... 28

Chapter 4 ............................................................................................................................... 29

Direct Compressive Measurements of Individual Titanium Dioxide Nanotubes ..... 29

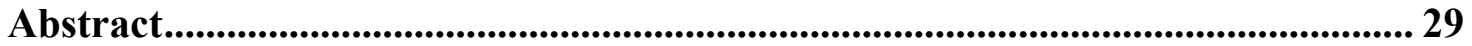

4.1 Introduction .................................................................................................................... 29

4.2. Experimental Procedure ................................................................................ 31

4.3. Results and Discussions .......................................................................................... 32

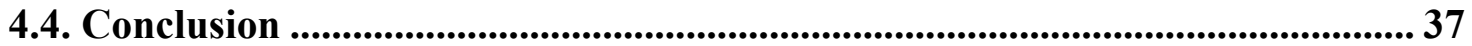

Acknowledgment ............................................................................................................... 37

Chapter 5 ............................................................................................................................... 38 
5.1. A Study on the Modulation of the Electrical Transport by Mechanical Straining

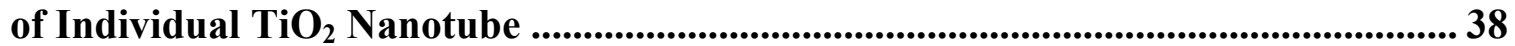

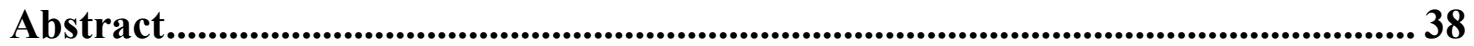

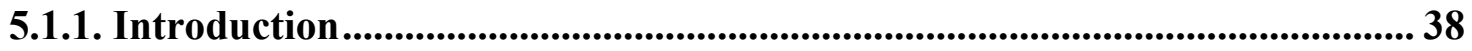

5.1.2. Experimental Procedure ............................................................................ 40

5.1.3. Results and Discussion .................................................................................. 40

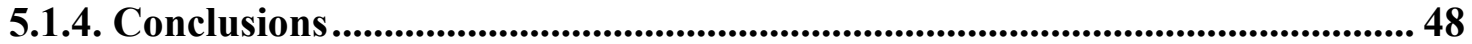

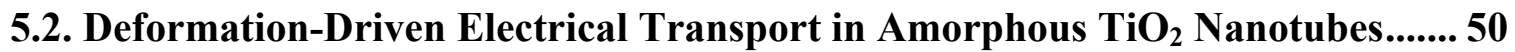

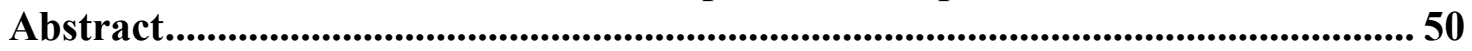

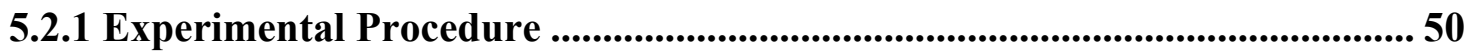

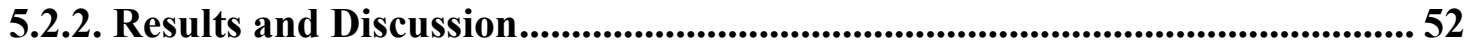

5.2.3. Conclusions ........................................................................................................ 57

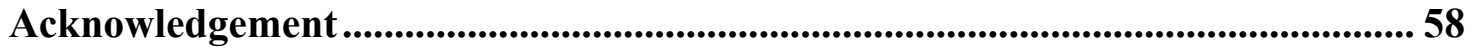

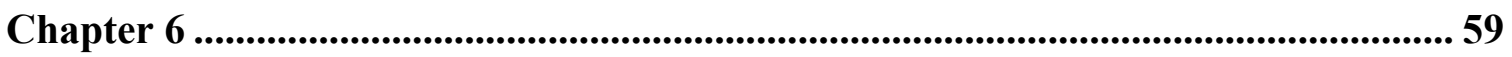

6.1. Structural Instabilities in $\mathrm{TiO}_{2}$ Nanotubes......................................................... 59

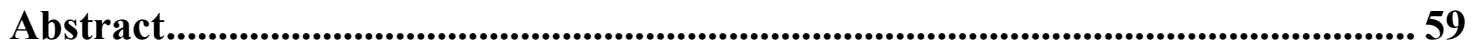

6.1.1. Introduction............................................................................................................. 59

6.1.2. Experimental Procedure ....................................................................................... 60

6.1.3. Results ................................................................................................... 61

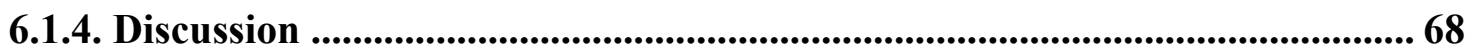

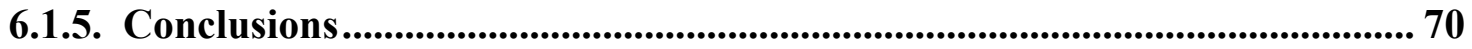

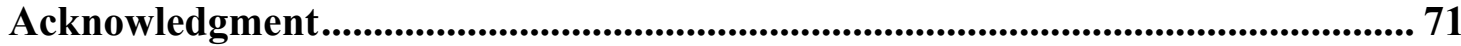

6.2. A Real Time Observation of Phase Transition of Anatase $\mathrm{TiO}_{2}$ Nanotubes Into Rutile Particles by In-Situ Joule Heating Inside Transmission Electron Microscope

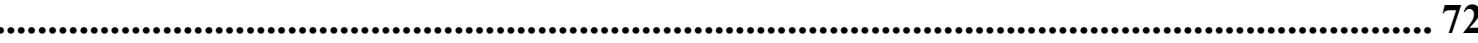

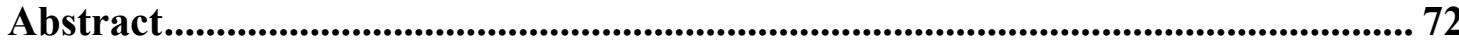

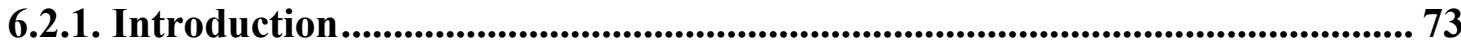

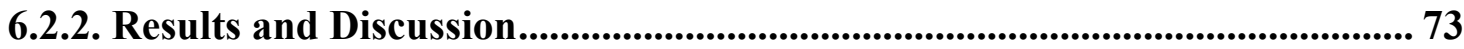

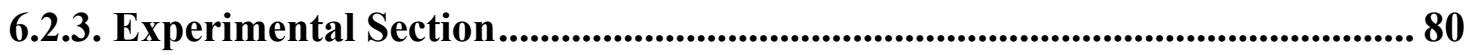

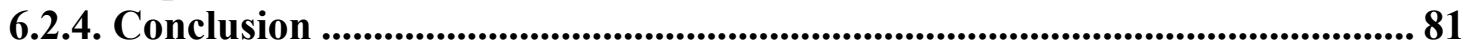

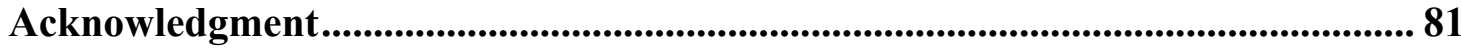

Chapter 7 ................................................................................................................................. 82

Droplet Wettability Changes of Titania Nanotube Surfaces Due to Annealing and

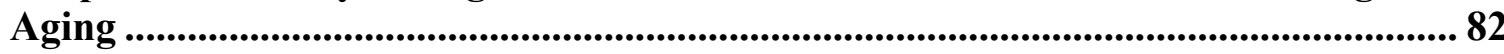

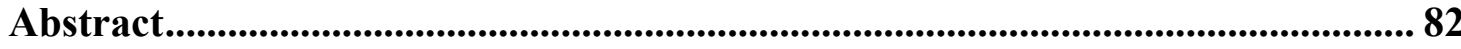

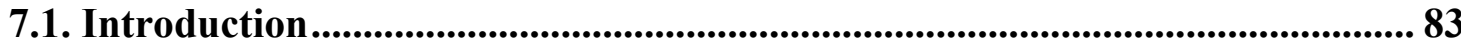

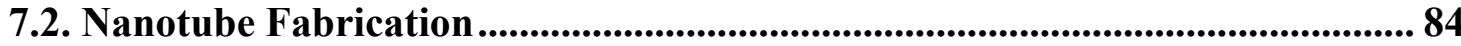

7.3. Contact Angle Measurements................................................................................. 87

7.4. Results and Discussion.............................................................................................. 89

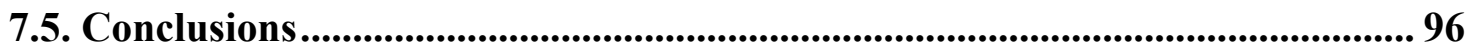

Acknowledgement .................................................................................................. 97

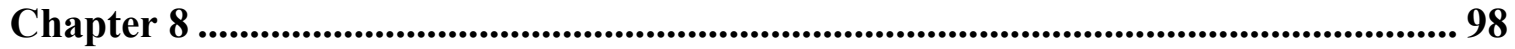


$\mathrm{TiO}_{2}$ Nanotubes Interaction with Osteoblasts ................................................................... 98

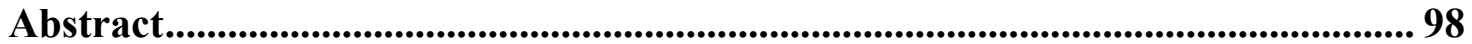

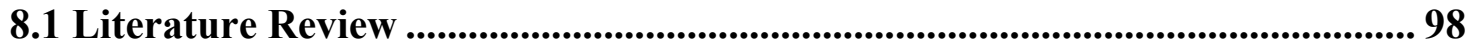

8.2. Experimental Procedure ..................................................................................... 100

8.2.1 Osteoblast Cell Culture Experiments........................................................... 100

8.2.2 Osteoblast Cell Viability Determination................................................. 101

8.2.3 Cell Number, Attachment and Spreading on Different Types of $\mathrm{TiO}_{2} \mathrm{Nanotube}$

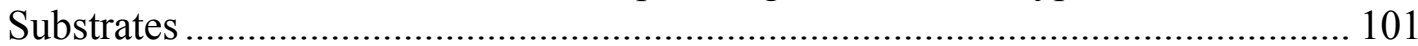

8.2.4 FIB Milling Procedure ............................................................................ 103

8.3. Effect of Nanotubes on Cell Attachment and Spreading ............................ 103

8.4. Effect of Nanotubes Topography, Wettability, Crystallinity, and Chemical

Composition on Osteoblast Cell Number .......................................................... 105

8.5. Preferred Directionality of Osteoblast Cell Growth....................................... 116

8.6. FIB Milling Studies at Cell-Nanotube Interfaces............................................ 117

8.7. Effect of Nanotube Stiffness on Cell Number.............................................. 123

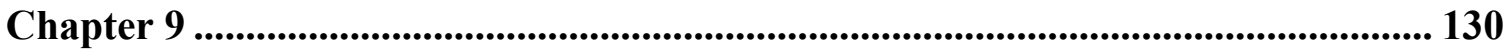

Discussion and Conclusions .......................................................................... 130

Chapter 10 ..................................................................................................................... 139

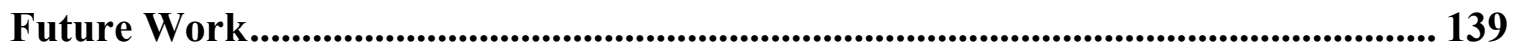

10.1 Evaluation of Osteoblast Cell Adhesion to $\mathrm{TiO}_{2}$ Nanotube Surfaces........... 139

10.2. Antibacterial Properties of Titania-Nanotube Surfaces............................... 140

10.3. Local Drug Therapy by Titania-Nanotube Array Surfaces.......................... 140

10.4. $\mathrm{TiO}_{2}$ Nanotubes for Cancer Therapy ............................................................ 141

10.5. Biocompatibility of $\mathrm{TiO}_{2}$ Nanotubes .......................................................... 142

Appendix ........................................................................................................... 143

Appendix 1: Copyright for the Manuscript Published in Applied Physics Letters

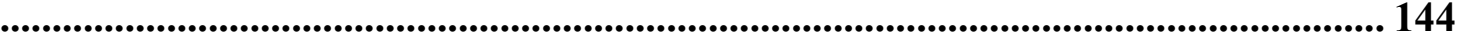

Appendix 2: Copyright for the Manuscript Published in Applied Science Letters

Appendix 3: Copyright for the Manuscript Published in Journal of Applied

Physics......................................................................................................... 146

Appendix 4: Copyright for the Manuscript Published in ACS Nano .................. 147

References........................................................................................................................... 148 


\section{List of Figures}

Figure 2.1. FE-SEM micrographs of Ti alloy surface treated with $\mathrm{H}_{3} \mathrm{PO}_{4}$ electrolyte : (a) $100 \mathrm{~V}$; (b) $150 \mathrm{~V}$; (c) $200 \mathrm{~V}$. Scale bar (a) is $5 \mu \mathrm{m}$, (b) is $500 \mathrm{~nm}$, and (c) is $2 \mu \mathrm{m} . . .9$

Figure 2.2. SEM micrographs of Ti-alloy anodized in $\mathrm{H}_{3} \mathrm{PO}_{4}$ and HF solution. (a and $\mathrm{b}$ ) no porous structure in $50 \mathrm{~V}$ and $100 \mathrm{~V}$ respectively; (c and d) a porous structure of 3 micron and 1 micron size was formed at 150 and $200 \mathrm{~V}$ respectively;(e and $\mathrm{f}$ ) a porous structure of $500 \mathrm{~nm}$. Scale bare $(\mathrm{a}, \mathrm{b}, \mathrm{c}, \mathrm{e})$ is $5 \mu \mathrm{m}$, and $(\mathrm{d}, \mathrm{f})$ is $2 \mu \mathrm{m}$.

Figure 2.3. SEM images of Ti surfaces treated with HF (a) $75 \mathrm{~V}$, (b) $100 \mathrm{~V}$, (c) $125 \mathrm{~V}$. Scale bar (a) is $5 \mu \mathrm{m}$, (b) is $3 \mu \mathrm{m}$, and (c) is $2 \mu \mathrm{m}$.

Figure 2.4. The effect of $\mathrm{H}_{3} \mathrm{PO}_{4}$ concentration at (a) $60 \mathrm{~V}$ and pore size range of 100-200 $\mathrm{nm}$; (b) $120 \mathrm{~V}$ and pore size range of $200-400 \mathrm{~nm}$; (c) $160 \mathrm{~V}$ and pore size range of $350-500 \mathrm{~nm}$; (d) $200 \mathrm{~V}$ and pore size range of 400-1100 nm.

Figure 2.5. Surface structure of Ti oxide obtained after sandblasting and (a.1, a.2, a.3) acid etching (SLA) of the rods. (b.1, b.2, b.3) electro etching (SLE) of the rods. The structure shows micro-nano porosity and significant roughness. Scale bar (a.1) is $500 \mu \mathrm{m},(\mathrm{a} .2, \mathrm{a} .3, \mathrm{~b} .3)$ is $200 \mu \mathrm{m},(\mathrm{b} .1)$ is $2 \mu \mathrm{m}$, and (b.2) is $10 \mu \mathrm{m}$.

Figure 2.6. SEM images of sandblasted and electro etched (SLE) rods. The structure shows micro-nano porosity and significant roughness. Scale bar (a) is $2 \mu \mathrm{m}$ and (b, c) is $5 \mu \mathrm{m}$.

Figure 3.1. I-T curves for 20,40 , and $60 \mathrm{~V}$ during anodizing process in $0.2 \mathrm{wt} \% \mathrm{NH}_{4} \mathrm{~F}$ solution.

Figure 3.2. FE-SEM images of the surface structure obtained and the calculation of diameter distribution at $40 \mathrm{~V}$ in $0.2 \mathrm{wt} \% \mathrm{NH}_{4} \mathrm{~F}$ at different times (a) 4, (b) 8, and (c) $16 \mathrm{hrs}$ and (d) diameter distribution with the average of 66,67 and $74 \mathrm{~nm}$ for 4, 8 and 16 hours under $40 \mathrm{~V}$.

Figure 3.3. FE-SEM images of the nanotubes at 16 hours in $0.2 \mathrm{wt} \% \mathrm{NH}_{4} \mathrm{~F}$ at (a) $20 \mathrm{~V}$, and (b) $60 \mathrm{~V}$. (c) Diameter distribution of nanotubes at 20, 40 and $60 \mathrm{~V}$ for 16 hours. The average diameter is 54, 74 and $144 \mathrm{~nm}$ for 20,40 , and $60 \mathrm{~V}$, respectively....... 24

Figure 3.4. At the voltages of 20, 40 and $60 \mathrm{~V}$, the inner diameter values are shown at anodization times of 4,8 and $16 \mathrm{hrs}$.

Figure 3.5. The microstructure nanotube obtained in $0.2 \mathrm{wt} \% \mathrm{NH}_{4} \mathrm{~F}$ at a voltage of $60 \mathrm{~V}$ and at (a) 4, (b) 8, and (c) $16 \mathrm{hrs}$. 
Figure 3.6. FE-SEM images of the microstructure obtained after $16 \mathrm{hrs}$ in $0.2 \mathrm{wt} \% \mathrm{NH}_{4} \mathrm{~F}$

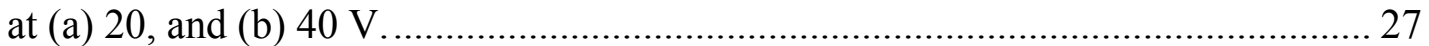

Figure 3.7. The nanotube lengths from 20,40 and $60 \mathrm{~V}$ at 4,8 and $16 \mathrm{hrs}$..................... 27

Figure 3.8. EDS analysis of a single alloyed (Ti6Al4V) nanotube indicating the Ti, Al,V, and $\mathrm{O}$ peaks, The EDS analysis confirmd that there was not any detectable extra element introduced to the rod surface during the formation of the nanotubes. ........ 28

Figure 4.1. FESEM images of a $\mathrm{TiO}_{2}$ nanotube array sample etched with a $2.0 \% \mathrm{HF}$ -

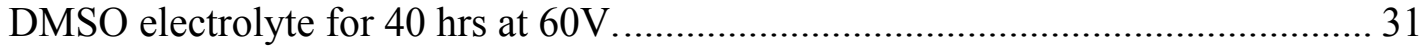

Figure 4.2. Schematic of the experimental setup within an AFM-TEM holder. The inset on the right shows a TEM view of a titanium dioxide nanotube being compressed between a sample wire and a silicon cantilever. The initial position of the tube against the cantilever may be precisely adjusted with piezo-driven displacement of the sample wire.

Figure 4.3. Force vs. displacement curves recorded for the thin-wall (top) and thick-wall (bottom) $\mathrm{TiO}_{2}$ nanotubes. The data from multiple experiments for each tube is overlaid in each plot with different color.

Figure 4.4. $\mathrm{A} \mathrm{TiO}_{2}$ nanotube fractured after application of maximum compressive load. The inset shows the collapse of the nanotube due to compressive load.................. 36

Figure 5.1. The image showing the STM-TEM holder used for present electrical measurements.

Figure 5.2. (a) An overall view of the anatase titania nanotube and the corresponding diffraction pattern (inset), and (b) the high resolution lattice image from a single anatase nanotube (inset).

Figure 5.3. Images from the FIB system show (a) the as grown $\mathrm{TiO}_{2}$ nanotube sample; (b) FIB probe attached with a single nanotube, dispersed on the $\mathrm{Cu}$ mesh; (c) the FIB probe with a nanotube approaching the tip of the tungsten wire and; (d) schematic of the experimental setup for current-voltage measurement. 42

Figure 5.4. The bight field image of the $\mathrm{TiO}_{2}$ nanotube (a) approaching the STM tip; (b) in contact with the STM tip; (c) (d) \& (e) undergoing a gradual increase in its bending curvature; (f) a series of the representable I-V curves measured during the deformation of the $\mathrm{TiO}_{2}$ nanotubes.

Figure 5.5. The $\ln I-V$ curves corresponding to (a) curve $b$ and (b) curve $c$ in Fig. 5.4f, respectively. (c) The linear fits of the curves in (a) and (b). 45 
Figure 5.6. The bight field image of the $\mathrm{TiO}_{2}$ nanotube (a) in a relaxed state after the unloading (b) The high resolution TEM image corresponding to the relaxed state (c) undergoing the bending deformation process beyond the elastic limit.

Figure 5.7. (a) TEM bright field (BF) image of the amorphous $\mathrm{TiO}_{2}$ nanotubes and its corresponding electron diffraction pattern (inset) and (b) the high-resolution lattice image.

Figure 5.8. Images from the FIB system showing (a) the as grown $\mathrm{TiO}_{2}$ nanotube sample; (b) FIB probe attached with a single nanotube, dispersed on the $\mathrm{Cu}$ mesh (c) the FIB probe with a nanotube approaching the tip of the tungsten wire and (d) Schematic of the experimental setup for current - voltage measurement. 52

Figure 5.9. The bight field image of the $\mathrm{TiO}_{2}$ nanotube $(a)$ approaching the STM tip; $(b)$ in contact with the STM tip; (c) (d) \& (e) undergoing a gradual increase in its bending curvature; (f) a series of the representable I-V curves measured during the deformation of the $\mathrm{TiO}_{2}$ nanotubes.

Figure 5.10. The $\ln I-V$ curves corresponding to (a) Curve $c$ and Curve $d$ in Fig. 5.9f, respectively. (c) The linear fits of the curves in (a) and (b)............................... 55

Figure 6.1. The SEM images of (a) as-synthesized $\mathrm{TiO}_{2}$ nanotubes arrays, and (b) close up of nanotubes shown in (a).

Figure 6.2. The bright field (a), diffraction pattern (b), and high-resolution TEM (c) images of as-synthesized $\mathrm{TiO}_{2}$ nanotubes.

Figure 6.3. $(\mathrm{a}, \mathrm{b})$ The SEM images of $\mathrm{TiO}_{2}$ nanotubes treated by $\mathrm{NH}_{4} \mathrm{OH}$ for $30 \mathrm{~min} . . . .63$

Figure 6.4. The SEM images show (a) as-synthesized $\mathrm{TiO}_{2}$ nanotubes after annealing in $500^{\circ} \mathrm{C}$. $(\mathrm{b}, \mathrm{c})$ The bright field TEM image and diffraction pattern of nanotubes shown in (a) indicate the transformation of amorphous morphology to crystalline order in the nanotubes. (d) The high resolution TEM image confirms the crystalline order of anatase phase and the presence of structural defects (appear as dark contrasts)..... 64

Figure 6.5. The SEM images show the morphology of the $\mathrm{NH}_{3}$-treated $\mathrm{TiO}_{2}$ nanotubes after calcination at $500^{\circ} \mathrm{C}$ for two hours. The treated nanotubes collapsed into nanoparticles upon heat treatment at anatase phase transformation temperature. .... 64

Figure 6.6. The SEM images of $\mathrm{NH}_{4} \mathrm{OH}$-treated $\mathrm{TiO}_{2}$ nanotubes after 0 (a), 10 (b), and 30 (c) minutes of calcination at $500^{\circ} \mathrm{C}$. The treated nanotubes start to disintegrate into small particles.

Figure 6.7. The XRD patterns of untreated and ammonium hydroxide (designated by $(\mathrm{N})$ ) $\mathrm{TiO}_{2}$ nanotubes 
Figure 6.8. The Raman spectroscopy of the Ti foil, untreated and ammonium hydroxidetreated $\mathrm{TiO}_{2}$ nanotubes. The $\mathrm{A}$ in parenthesis stands for anatase.

Figure 6.9. Bright field TEM images of nanoparticles after the collapse of $\mathrm{TiO}_{2}$ nanotubes by $\mathrm{NH}_{3}$-treatment and annealing at $500^{\circ} \mathrm{C}$. The inset shows the diffraction pattern of particles and indicates that the crystal structure is anatase..... 68

Figure 6.10. (a) The as-synthesized $\mathrm{TiO}_{2}$ nanotubes show imperfections on the surface (marked by the arrows). (b) The elastic modulus map of $\mathrm{TiO}_{2}$ nanotubes before immersion in the ammonia hydroxyl media is shown. The dark brownish color indicates areas of high stiffness and areas of bright yellowish correspond to low stiffness values. The scanned area is $500 \mathrm{~nm}$ and the color key represents qualitative comparison of the modulus.

Figure 6.11. A schematic of the observed structural instability in $\mathrm{TiO}_{2}$ nanotubes treated with $\mathrm{NH}_{4} \mathrm{OH}$ and calcinated at $500^{\circ} \mathrm{C}$. The surface roughness is shown in the larger scale than the nanotube dimension. The nanotube disintegrated into anatase particles after 10 and 30 min of calcination but maintained the overall configuration of nanotube. After $2 \mathrm{hr}$ the nanotubes collapsed into nanoparticles.

Figure 6.12. (a) TEM bright field (BF) image of the as-synthesized $\mathrm{TiO}_{2}$ nanotubes and its corresponding electron diffraction pattern (inset) and (b) the high resolution lattice image.

Figure 6.13. (a) An over all view of the anatase titania nanotube and the corresponding diffraction pattern (inset) and (b) the high resolution lattice image from a single anatase nanotube (inset).

Figure 6.14. Images from the FIB system showing (a) the as grown $\mathrm{TiO}_{2}$ nanotube sample; (b) FIB probe attached with a single nanotube, dispersed on the $\mathrm{Cu}$ mesh (c) the FIB probe with a nanotube approaching the tip of the tungsten wire and (d) Schematic of the experimental setup for current - voltage measurement. 76

Figure 6.15. The BF image of the $\mathrm{TiO}_{2}$ nanotube (a) in contact with the STM tip (b) undergone the heat treatment at constant bias voltage of $10 \mathrm{~V}$ for $180 \mathrm{sec}$ (c) the bias heated at $20 \mathrm{~V}$ for $130 \mathrm{sec}(\mathrm{d})$ undergone the bias heating at $30 \mathrm{~V}$.

Figure 6.16. The current (I) versus time (t) plot for the $\mathrm{TiO}_{2}$ nanotube under the different bias heating voltages, as marked in the plot.

Figure 6.17. (a) The high resolution lattice image and the corresponding diffraction pattern (inset) taken from $\mathrm{TiO}_{2}$ nanotube undergone a bias heating at $10 \mathrm{~V}$ (b) the high resolution lattice image of the nanotube bias heated at $20 \mathrm{~V}$ and the corresponding diffraction pattern (inset) (c) The diffraction pattern from the agglomeration formed at the bias heating of $30 \mathrm{~V}$. 
Figure 7.1. Electrochemical etching (anodization) setup for the $\mathrm{TiO}_{2}$ nanotube fabrication. The anodization setup consisted of a Ti foil, a Pt counter electrode, a voltage source, and a themometer. The nanotubes were etched on the Ti foil substrate.

Figure 7.2. Titanium dioxide nanotubes prepared by Pt electrode $(\mathrm{a} \sim \mathrm{f})$. In general, titania nanotubes had lengths bewteen 7 10 microns and inner diameters ranging from 60 130 $\mathrm{nm}$. (a) and (b) are amorphous, (c) and (d) are crystalline, (e) is assythesized, and (f) is annealed TNT.

Figure 7.3. TEM images of TNT structured surfaces (a) as-sythesized nanotubes are amorphous, (b) annealed nanotubes are crystalline.

Figure 7.4. Schematic of the experimental setup. Lens array consisted of (a) diffuser, (b) bi-convex lens, (c) large aperture, (d) small aperture, (e) plano-convex (KPX079), and (f) plano-convex (KPX088).

Figure 7.5. (above) Representative image comparison of the initial contact angles. Six samples in total were investigated. (below) Results of the contact angles on the surfaces. The TNT structures made the surface more hydrophilic and the annealing effect made the TNT surface super-hydrophilic. Contact angle differences were attributed to the surface properties, in particular the surface roughness, which could affect the initial contact angle of a water droplet on each titanium surface.

Fig. 7.6. Comparison of the time-dependent change of TNT surface wettability. As time passed, the TNT surface hydrophobicity increased.

Figure 7.7. Comparison of the time-dependent contact angles, (a) shows the water droplet wettability on the annealed and as-anodized TNT surfaces. The increasing contact angles varied in each case but all of the TNT samples had the tendency of increasing the surface wettability. (b) shows the cases of bare titanium. In these cases, the wettability had little, if any, change much with time.

Figure 7.8. FTIR spectroscopy results for annealed and aged, as-anodized and aged, and as-anodized fresh TNT surfaces. It was confirmed that the fresh TNT surfaces had more hydroxyl groups on the surface than the aged TNT. The vertical line corresponds to approximately $3300 \mathrm{~cm}^{-1}$

Figure 8.1. Cell viability determination by microscopic imaging and observation of cell activity such as number, attachment as spreading after (A): no cell spreading; $1 / 2 \mathrm{hr}$, (B): partially spread cells; $2 \mathrm{hrs,} \mathrm{(C)} \mathrm{fully} \mathrm{spread} \mathrm{cells;} 48 \mathrm{hrs}$. The field of view is $400 \times 300 \mu \mathrm{m}^{2}$. 
Figure 8.2. (a-c) Contact angle measurements for the cp- $\mathrm{TiO}_{2}$ nanotube surface, (a) annealed cp-Ti surface, (b) not annealed cp-Ti surface, (c) bare cp-titanium surface. (d-f) (d) Contact angle measurements for the Ti6AL4V nanotube surface, annealed Ti6Al4V, (f) not annealed Ti6Al4V nanotube surface (e), bare Ti6Al4V surface. 106

Figure 8.3. SEM micrograph of MC3T3 cells after $24 \mathrm{hrs,} 48 \mathrm{hrs}$ and $72 \mathrm{hrs}$ of culture on control cp-Ti (a-c) and as anodized amorphous cp- $\mathrm{TiO}_{2}$ nanotubes (d-f). Cell number increases as a function of culture time in both control and anodized samples. The number of attached cells increases by anodization (d-f). Scale bar (a, b, d, e) is $200 \mu \mathrm{m}$, and ( $\mathrm{c}, \mathrm{f})$ is $500 \mu \mathrm{m}$. 108

Figure 8.4. SEM micrograph of MC3T3 cells after $24 \mathrm{hrs}, 48 \mathrm{hrs}$ and $72 \mathrm{hrs}$ of culture on annealed $\mathrm{cp}-\mathrm{TiO}_{2}$ nanotubes (a-c). Cell number increases as a function of culture time. The number of attached cells increases by annealing (d). Scale bar (a) is 200 $\mu \mathrm{m},(\mathrm{b}, \mathrm{c})$ is $500 \mu \mathrm{m}$.

Figure 8.5. SEM micrograph of MC3T3 cells after $24 \mathrm{hrs}, 48 \mathrm{hrs}$ and $72 \mathrm{hrs}$ of culture on control Ti6Al4V (a-c) and as-anodized amorphous alloyed- $\mathrm{TiO}_{2}$ nanotubes (d-f). Cell number increased as a function of culture time in both control and anodized samples. The number of attached cells increased by anodization (d-g). Scale bar $(\mathrm{a}, \mathrm{d}, \mathrm{e})$ is $200 \mu \mathrm{m},(\mathrm{b}, \mathrm{c})$ is $100 \mu \mathrm{m}$, and (f) is $500 \mu \mathrm{m}$.

Figure 8.6. SEM micrograph of MC3T3 cells after $24 \mathrm{hrs}, 48 \mathrm{hrs}$ and $72 \mathrm{hrs}$ of culture on annealed alloyed $\mathrm{TiO}_{2}$ nanotubes (a-c). Scale bare (a) is $200 \mu \mathrm{m}$ and (b,d) is 500 $\mu \mathrm{m}$.Cell number increases as a function of culture time. The number of attached cells increases by annealing (d). Scale bar (a) is $200 \mu \mathrm{m}$, and (b,c) is $500 \mu \mathrm{m} . \ldots . .112$

Figure 8.7. Number of adhered cells as a function of incubation time on the surface of bare $\mathrm{Ti}$ (left) and $\mathrm{TiO}_{2}$ nanotubes (right). The data of this chart has been obtained from comparison and combining the graphs of Figures 8.4d and 8.6d. The data confirms the effect of anodization and annealing in enhancing number and growth in comparison to control bare surfaces for both $\mathrm{cp}$ and alloyed samples. However, in the cell count of annealed samples there is a slight increase in the case of $\mathrm{cp}$ nanotubes compared to alloyed nanotubes which could be due to higher biocompatibility of $\mathrm{cp}$ Ti.

Figure 8.8. The effect of nanotube length, crystallinity and composition on cell number and attachment. Scale bar (a,b) is $500 \mu \mathrm{m}$, and (c,d,e,f) is $200 \mu \mathrm{m}$.

Figure 8.9. SEM micrographs of osteoblast cells when cultured on $\mathrm{TiO}_{2}$ nanotubes: random cell orientation on control Ti sample without nanotubes (a) parallel cell orientation to the nanotube direction (b-c-d). Scale bare for (a-b-d) is $50 \mu \mathrm{m}$ and for (c) is $100 \mu \mathrm{m}$. 
Figure 8.10. (a-b) FIB milled fibroblast cells obtained from a primary ion beam. Internal structures, membranes and morphological stability and integrity can be assessed; moreover the interface between the cell and the substrate can be observed. Scale bar (a) is $5 \mu \mathrm{m}$, and (b) is $10 \mu \mathrm{m}$. 119

Figure 8.11. SEM images of the $(a, b)$ cross section view of cell-nanotube interface, milled by FIB technique and (c, d) magnified view of cell-nanotube interaction and filopodia growth inside nanotube. Scale bar (a) is $2 \mu \mathrm{m}$, (b) is $1 \mu \mathrm{m}$, and (c,d) is 500 $\mathrm{nm}$.

Figure 8.12. Osteoblast cell interaction with $\mathrm{TiO}_{2}$ nanotubes is shown. The extension of osteoblast cells for adhesion on the nanotubes as well as cell clusters (a) on the nanotube surface, indicate the ability of the nanotube structure to promote cell adhesion, and growth can be observed. Scale bar (a) is $100 \mu \mathrm{m}$, (b) is $50 \mu \mathrm{m}$, and (c) is $10 \mu \mathrm{m}$.

Figure 8.13. Cell filopodia attachment and spreading on and in between nanotubes. The nanotubes can act as anchors for cell filopodia to grab (f) and increase the spreading (d) and improve its attachment (e). Scale bar (d) is $3 \mu \mathrm{m}$, (e) is $500 \mathrm{~nm}$, and (f) is 1 $\mu \mathrm{m}$.

Figure 8.14. FIB cross sectional milling (A-D) revealing the clogged titania nanotube by osteoblast and calcium deposition on nanotubes (F-F) as a result of cell attachment and direct contact with surface. Scale bar (A) is $200 \mu \mathrm{m},(\mathrm{B})$ is $50 \mu \mathrm{m},(\mathrm{C})$ is $20 \mu \mathrm{m}$, $(\mathrm{D}, \mathrm{F})$ is $5 \mu \mathrm{m}$, and $(\mathrm{E})$ is $10 \mu \mathrm{m}$. 122

Figure 8.15. EDS analysis of the high-resolution scanning electron micrograph of clogged nanotubes. The clogged nanotubes are visible and composed of $\mathrm{Ca}$ and $\mathrm{P}$ (EDS), the primary components of bone matrix. Scale bar is $1 \mu \mathrm{m}$.

Figure 8.16. ECM production on and in between nanotubes during cell migration and bone matrix deposition. Scale bar left is $5 \mu \mathrm{m}$ and right is $1 \mu \mathrm{m}$.

Figure 8.17. The vertically-aligned $\mathrm{TiO}_{2}$ nanotubes that are anodized on the Ti implant surface can be modeled as a series of cantilever springs with spring constants of $\mathrm{K}_{1}$, $\mathrm{K}_{2}, \mathrm{~K}_{3}, \ldots \mathrm{K}_{\mathrm{n}}$

Figure 8.18. The normalized $K a$ parameter as a function of nanotube length, $L$, is plotted. The nanotubes were synthesized at three different applied voltages, 20, 40, and 60 V.

Figure 8.19. The normalized $K a$ parameter as a function of anodization duration, $T$, is plotted. The nanotubes were synthesized at three different applied voltages, 20, 40, and $60 \mathrm{~V}$.

Figure 8.20. Effect of nanotube stiffness on cell number is plotted for $\mathrm{TiO}_{2}$ nanotubes anodized at $60 \mathrm{~V}$. The higher the stiffness, the better the cell growth. 128 


\section{List of Tables}

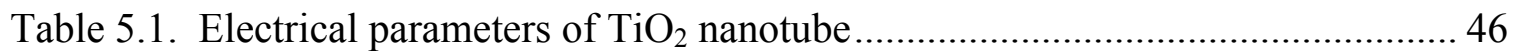

Table 5.2. Electrical parameters of $\mathrm{TiO}_{2}$ nanotube .................................................. 56

Table 7.1 Variation of contact angle with respect to time ......................................... 93 


\section{Chapter 1}

\section{Introduction}

After the implantation of a biomaterial in the body, the interaction of the biomaterial with cells initially occurs in the surface of the implant. ${ }^{1}$ Thus, it is possible to modulate the biological responses of the body by modifying the implant's surface characteristics. During the past few decades, many implanted biomaterial failures have been reported due to weak surface properties and chronic infections. Several surface modifications have been suggested to overcome the implant failures associated with insufficient tissue-implant interaction due to the lack of suitable surface properties. In the case of titanium orthopedic implants, it has been widely accepted that the biocompatibility of a titanium implant is attributed to the passive oxide layer $\left(\mathrm{TiO}_{2}\right)$ formed on its surface. Therefore, understanding the surface properties of titanium and titanium alloy implants such as roughness, chemistry, wettability, and mechanical properties are crucial for designing a successful implant for clinical application. However the mechanism in which these surface properties regulate cell function is not clearly understood. This thesis will mainly focus on $\mathrm{TiO}_{2}$ nanotube synthesis, characterization and osteoblast interaction as the next generation surface modification of titanium and titanium alloy implants.

In Chapter 2, a review of my work on surface modification of titanium alloys through porosification via hydrofluoric acid is given. This technique has several limitations including working with hazardous acids, long times required for etching, and lack of control over the size distribution of pores. I will compare several etching techniques including a method based on sandblasting as the pretreatment. I then focused on the application of different methods for surface modification. In Chapter 3, I introduce the electrochemical synthesis of $\mathrm{TiO}_{2}$ nanotubes at the surface of bulk Ti. The nanotubes resemble a tubular nanostructure. This method does not require working with hazardous acids such as HF. In turn, the sizes of the nanotubes can be controlled to a great extent by adjusting the synthesis parameters such as applied bias voltage, time, and chemistry of 
the electrolyte. Discussion on the effect of anodization parameters on nanotube architecture is given in Chapter 3.

These engineered-nanotubular structures offer exciting progress toward the design of multifunctional medical implants. To bring this to reality, one should know the mechanical, electrical, surface, and biocompatibility properties of the nanotubes. The mechanics of the nanotubes is important from stress-shielding and mechano-transduction points of view. I speculated that surface modification of Ti implants by a $\mathrm{TiO}_{2}$ nanotube layer with elastic behavior close to the actual bone can be promising to overcome stressshielding, a common reason for implant failures. In addition, I hypothesize that the fabrication of $\mathrm{TiO}_{2}$ nanotubes with elastic modulus close to actual bone can promote osteoblast growth. Interestingly, no previous reports in the body of literature could be found on the mechanical properties of nanotubes. This motivated me to conduct series of novel experiments on measuring the mechanical properties of individual $\mathrm{TiO}_{2}$ nanotubes. The nanotubes were tested in the chamber of transmission electron microscope using an in-situ atomic force microscopy stage with force resolutions better than $1 \mathrm{nN}$. Thin and thick nanotubes were tested to check if there are variations of mechanical properties as a function of size. All the data and relevant discussion related to the mechanical testing of nanotubes are given in Chapter 4.

The motivation to study the electrical properties of $\mathrm{TiO}_{2}$ nanotubes is based on the work of Ercan et al. ${ }^{2}$ who showed that application of an electrical signal on anodized nanotubular titanium can significantly improve osteoblast proliferation and differentiation. However, I could not find any reports in the literature, on the electrical properties of individual nanotubes. There are a number of reports on the electrical performance of arrays of nanotubes but no single nanotube was tested. Biological cells are normally nano-micro sized and have interactions with nanotubes at the nanoscale. Therefore it is important to measure a nanotube electrical conductivity at the nanoscales. In addition, it wasn't known how differently the electrical properties of an as-anodized (amorphous) versus annealed (crystalline) nanotube can be. This motivated me to characterize the electrical properties of amorphous and crystalline nanotubes as discussed in Chapter 5. 
II addition, biological cells attached to the surface of nanotubes can mechanically bend the nanotubes. However, we do not know how this mechanical straining can impact the electrical signal transmission in the nanotubes. This motivated me to study the effect of mechanical straining on the electrical properties of nanotubes. It was observed that mechanical straining can enhance the electrical properties of nanotubes by a factor that should be considered during electrical signaling of the cells. The results are also explained in Chapter 5.

If the nanotubes are used on the surfaces of implants they will be subjected to body temperature in the presence of the physiological environment. Therefore it is important to investigate the conditions in which the $\mathrm{TiO}_{2}$ nanotubes may become structurally unstable. In addition in the process of implant fabrication may be certain industrial treatments that may require annealing the implant in a nitrogen concentrated environment (nitriding). Investigating the treatment conditions that may result in nanotube breakdown is of high importance for optimizing the annealing parameters. This has been addressed in Chapter 6 for understanding factors behind the annealing process that are to be avoided or to be aware of. In this chapter, another investigation is devoted to the Joule heating of nanotubes that are subjected to electrical signaling. I observed that the nanotubular structure can collapse into small particles if subjected to high electrical biasing over a period of time. This consideration opens new discussions and future efforts on what regime the electrical methods for regulating the cell functions can be effective before damaging the nanotube architectures.

After the implantation of a biomaterial in the body, the biological interaction of the biomaterial with cells initially occurs on the surface of the implant. The immediate contact of the tissue with $\mathrm{TiO}_{2}$ nanotubes starts with water molecules, making the wettability properties of the nanotubes a high impact factor in protein adsorption. ${ }^{3}$ Since cells are connected to matrix proteins by integrins, and hydrophilicity of the surface encourages protein adsorption, hence surfaces with higher hydrophilic properties can enhance cell adhesion. These findings motivated me to investigate the wettability properties of $\mathrm{TiO}_{2}$ nanotubes and to investigate $\mathrm{TiO}_{2}$ nanotube hydrophilicity based on the nanotube composition and crystallinity (Chapter 7). Interestingly, I observe that the 
wettability can be inversely affected by an annealing treatment and then by aging of the nanotubes.

In Chapter 8, I further extended the existing knowledge in the literature by investigating the effect of chemical composition, crystallography, and stiffness of $\mathrm{TiO}_{2}$ nanotubes on osteoblast cell function. I have shown that the nanotubular characteristics of the surface improve cell proliferation, attachment and spreading of osteoblast cells. I also investigated the effect of the chemical composition on osteoblast growth and adhesion by comparisons between alloyed and pure. Moreover, osteoblast interaction and attachment with $\mathrm{TiO}_{2}$ nanotubes was investigated through focused ion beam cross sectional milling to reveal osteoblast growth into nanotubes. Finally I have proposed a method for evaluating the effect of nanotube stiffness on cell attachment and number and therefore to perhaps increase the morphological properties of the nanotubes for better cell adhesion and proliferation.

The work outlined above has resulted in a new understanding of the behavior of these nanomaterials and their interactions with osteoblast cells. However, several problems or issues should be addressed before they can be used as medical implants. This work can be extended in several directions to make its application feasible. One concern is the delamination of $\mathrm{TiO}_{2}$ nanotubes from the surface during implant loading in the body and if they have adverse effects on kidneys or other human organs. Another extension of this work will be toward growth factor and drug loading and release. Although I reported the electrical properties of the nanotubes, the in-vivo investigation of electrical signaling coupling with $\mathrm{TiO}_{2}$ nanotubes to regulate cell function remains unexplored. Moreover, the photothermal properties of $\mathrm{TiO}_{2}$ nanotubes as a potential therapeutic agent for cancer thermotherapy in combination with near-infrared (NIR) light has been reported to be an affective way to destroy cancer cells. ${ }^{4}$ However, the potential that $\mathrm{TiO}_{2}$ nanotubes can inhibit tumor growth remains unexplored. Therefore, in-vivo animal tests should be conducted to investigate if the $\mathrm{TiO}_{2}$ nanotubes can inhibit tumor growth with any possible IR-induced photocatalytic side effects. All these ideas and possible extensions of this $\mathrm{PhD}$ research work are discussed in Chapter 9. 


\title{
Chapter 2
}

\section{SLE Technique for Surface Engineering of Titanium Rods with Modified Porous Structures}

\begin{abstract}
It is generally known that surface porosity and roughness on titanium substrates can enhance osteoblast adhesion, proliferation, production of alkaline phosphates, and deposition of calcium-containing mineral. ${ }^{5}$ This research aims to perform an initial study on the potential surface modifications of titanium alloys used as implants and to develop a better understanding on the effect of anodization parameters on the structure of surface porosities. Surface roughness with micro- and nano-sized pores was produced through a novel technique that included sandblasting and electrochemical etching processes (SLE). A systematic study was conducted to compare the SLE results with conventional techniques including sandblast-acid-etched (SLA), and selective electrochemically etched (SEE) processes. The SEE experiments were conducted under four different process conditions using hydrofluoric and phosphoric acids. The morphology comparison between these three surfaces indicated that the SLE surface had a range of micro-nano scale surface porosity. These structural features can potentially be applied for titanium implants to improve the bonding between the orthopedic implants and surrounding bone matrix.
\end{abstract}

However, during this work I noticed a number of serious drawbacks of SLA, SLE and SEE techniques. First, the fabrication involves the use of extremely hazardous chemicals such as HF acid. Handling HF requires extreme caution and expensive equipment for protection. Second, the process of making micro porosity is very time consuming (e.g. up to $96 \mathrm{hrs}$ ). Interestingly, the fabrication of nanotubular morphology does not have any of the above-mentioned disadvantages. In addition, the literature shows that protein adsorption and consequent cell adhesion and proliferation is higher on nanotubular modified titanium surfaces compared to porous surfaces due to 
enhanced roughness, more surface area, and therefore higher surface energy for reaction with protein molecules. These reasons motivated me to look for an improved surface treatment by nanotubular surface modification of titanium implants using the initial principals of anodization but safer electrolyte and a time/cost effective protocol. The fabrication of nanotubular structures is discussed in Chapter 3.

\subsection{Introduction}

In recent years, the use of titanium (Ti) and Ti alloys as materials for implants has been increasing in comparison to many other metals. Researchers believe that increased knowledge of titanium and other materials, and the body's reaction to them, could make permanent surgical implants the norm. This could herald a new area of dental and orthopedic work, with immediate applications in dentistry, reconstructive surgery, and hip, knee, finger and ankle replacements. The interest in Ti implants has been attributed to the superior biocompatibility provided by the thin oxide surface layer $(1.5-10 \mathrm{~nm}$ thick) that forms naturally on the surface of titanium metal on exposure to air. ${ }^{6}$

This bond between the implant and bone (osseointegration) is being actively studied. In fact, improved bonding between the bone and the titanium implant was observed on microporous structures compared with non-microporous titanium which did not induce bone formation at all. ${ }^{7}$ In addition to topography, implant surface chemistry plays an important role in protein adsorption and subsequent cell adhesion. The surface oxide layer of titanium has many qualities regarded as important for promoting bone growth. This oxide layer of titanium can be manipulated chemically and there has been speculation whether the biological properties of the oxide surface may then be changed and even improved. In addition the ability to control drug delivery at the surface of a titanium rod implant can promote bone growth and infection resistance.

Researchers are experimenting with Ti with different surfaces that may encourage early bone build-up. The results show that rough surfaces allow bone to grow into them more actively. ${ }^{8}$ Surface roughness is an immensely studied subject by numerous investigators with the goal of improving the performance of bone implants. Specifically, compared with smooth surfaces, micron surface roughness on titanium substrates 
created by sandblasting, etching, machining and the use of micron-sized metal bead coatings has enhanced osteoblast adhesion, proliferation, production of alkaline phosphates, and deposition of calcium-containing mineral. ${ }^{9}$ Thus, in order to understand the bone growth process between the implant and bone tissue, it is very important to know the surface properties of the film. For this reason, many investigations of tissue reactions to implant surfaces have been directed at modifying the roughness of the thin film surface and most efforts have been concentrated on the surface technology of implants, and roughened surfaces were demonstrated to favor bone bonding of implants, ${ }^{10,11,12}$ although the optimal surface modification has not yet been found. Consequently, a variety of techniques and implant surfaces were developed and introduced to the implant market. Among the techniques, those that produce an increase in the surface roughness and form a $\mathrm{TiO}_{2}$ layer on the surface have been found to be beneficial to the biological performance of the implants. ${ }^{13}$

Another new developed and well-documented surface treatment of Ti implants has been known as sandblasted and acid-etched surface (SLA), which consistently showed better results both in histometric and biomechanical testing in comparison with alternative surfaces, ${ }^{14,15,16}$ such as the well documented TPS (titanium plasma-sprayed) surface, and furthermore proved advantageous in clinical application. ${ }^{17}$ Most importantly, it can reduce the routine 12-week unloaded bone healing time to 6 weeks, allowing an early loading. ${ }^{18,19} \mathrm{Li}$ et al. ${ }^{20}$ provided a study with the purpose to biomechanically compare these two subtly different surfaces, the machined and acid-etched surface (MA) and SLA, using an identical implant design and to further demonstrate the interlocking effect of the macro-rough texture produced by the sandblasting procedure before acid etching. Through their direct biomechanical comparison study, the SLA was demonstrated to be more powerful in enhancing the interfacial shear strength of implants than the MA surface. In comparison with the MA-surfaced implants, the SLA-surfaced implants showed approximately $30 \%$ higher removal torque values (RTVs) at the healing periods of 4,8 , and 12 weeks and also a more than $5 \%$ higher interfacial stiffness. The preprocessing by sandblasting before acid etching accounts for these differences and therefore it was clarified that the sandblasting as a preprocessing treatment is 
necessitated as well from the viewpoint of biomechanics.

All the investigations cited above were concerned with the development of the titanium oxide layers, the reduction of the bone healing period and, consequently, with enhancing a patient's life. In this research for the first time, we report a new technique that includes sandblasting and electrochemical etching (SLE). We then conducted a systematic microstructural study on the formation of various nano/microstructures of titanium oxide produced by SEE, SLE, and SLA processes.

\subsection{Experimental Procedure}

The samples were titanium alloy Ti6Al4V rods, $2.5 \mathrm{~mm}$ in diameter and $5 \mathrm{~cm}$ in length. The rods were first machined and then prepared by cleaning in an acetone ultrasonic bath followed by an isopropyl alcohol wash. The rods were then rinsed in DI water and dried in a nitrogen stream. The surface structure of the samples was analyzed by a Hitachi S-4700 field emission scanning electron microscope (FE-SEM).

The first sets of experiments, selective electrochemical etchings (SEE), were conducted under four different process conditions using hydrofluoric and/or phosphoric acids in distilled water as the electrolyte media. Voltage, time, and electrolyte concentration were varied to improve the desirable porous structure. The next sets of experiments were conducted by acid etching and electro etching of the sand blasted samples. I called these two techniques SLA and SLE respectively. Micro abrasive of 50$100 \mu \mathrm{m}$ were used for the sand blasting process. During the SLA process the titanium rod was acid etched for $72 \mathrm{~h}$ in $\mathrm{H}_{2} \mathrm{SO}_{4}$ and then $94 \mathrm{~h}$ in $\mathrm{HCl}$. The electrochemical etching of the SLE process was conducted under the etch conditions obtained from the selective electrochemical etching experiments.

\subsection{Results}

\subsubsection{Selective Electrochemical Etching (SEE) Process}

It has been found that the morphology of titanium oxide is dramatically changed upon electrochemical conditions. ${ }^{21}$ The mechanism of nanostructured titanium 
oxides in terms of different anodizing conditions have been described by a competitive reaction between $\mathrm{F}^{-}$ions and $\mathrm{PO}_{4}{ }^{3-}$, which provides different levels of incorporation of anions in the formed oxide. Titanium oxides containing enriched $\mathrm{F}^{-}$ions stimulate cell attachment, whereas high proliferation levels of cells were observed in phosphate incorporated titanium oxides.

Voltage, time and electrolyte concentration were varied in four different sets of experiments to obtain the processing conditions for the desirable porous structure.

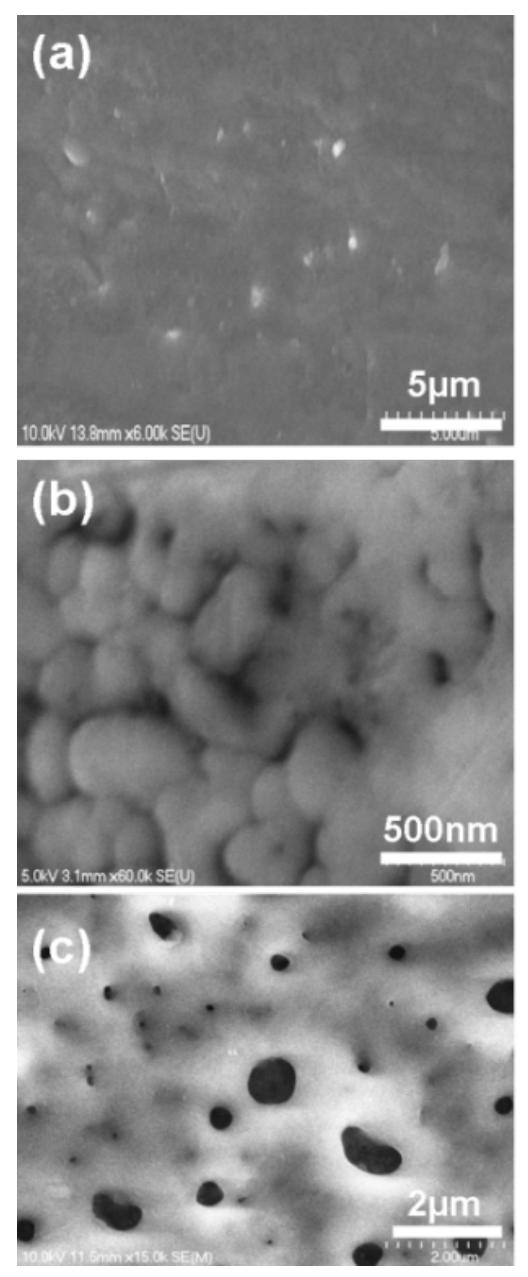

Figure 2.1. FE-SEM micrographs of Ti alloy surface treated with $\mathrm{H}_{3} \mathrm{PO}_{4}$ electrolyte : (a) $100 \mathrm{~V}$; (b) $150 \mathrm{~V}$; (c) $200 \mathrm{~V}$. Scale bar (a) is $5 \mu \mathrm{m}$, (b) is $500 \mathrm{~nm}$, and (c) is $2 \mu \mathrm{m}$. 


\subsubsection{Effect of Voltage with $\mathrm{H}_{3} \mathrm{PO}_{4}$ Electrolyte}

This electrochemical etching was carried out at room temperature using $1 \mathrm{M}$ $\mathrm{H}_{3} \mathrm{PO}_{4}$ electrolyte. The process was for $15 \mathrm{~min}$ at 50, 100, 150, and $200 \mathrm{~V}$. In general, the porosity and the pore size increased with increasing voltage (Figure 2.1). The nanoporous titanium layers were detectable at voltage values of $150 \mathrm{~V}$ and $200 \mathrm{~V}$ but no porous structure was observed for 50 and $100 \mathrm{~V}$. The surface morphology in the sample formed with $200 \mathrm{~V}$ had a distinct porous structure in comparison to other samples processed at lower voltages.

\subsubsection{Effect of Voltage with $\mathrm{HF}+\mathrm{H}_{3} \mathrm{PO}_{4}$ Electrolyte}

Subsequently, hydrofluoric acid (HF) was added into the $\mathrm{H}_{3} \mathrm{PO}_{4}$ electrolyte for the study of the electrolyte composition effect and applied voltage on the morphologies of titanium oxides. Anodic oxidation was carried out at room temperature using $1 \mathrm{M}$ $\mathrm{H}_{3} \mathrm{PO}_{4}+1 \mathrm{wt} \% \mathrm{HF}$ electrolyte. It was found that the morphology of titanium oxides were significantly influenced by composition of the electrolyte and applied potential. The process was carried out for $15 \mathrm{~min}$ at 50,100,135, 150 and $200 \mathrm{~V}$. It was observed that a nanoporous structure with pore size range of $200-500 \mathrm{~nm}$ was formed at $135 \mathrm{~V}$ (Figures $2.2 \mathrm{e}$ and 2.2f) and a combination of micro and nano porous structures were formed at 150 $\mathrm{V}$ and $200 \mathrm{~V}$ with average pore sizes of 3 and 1 micron respectively (Figures 2.2.c and $2.2 d)$. 

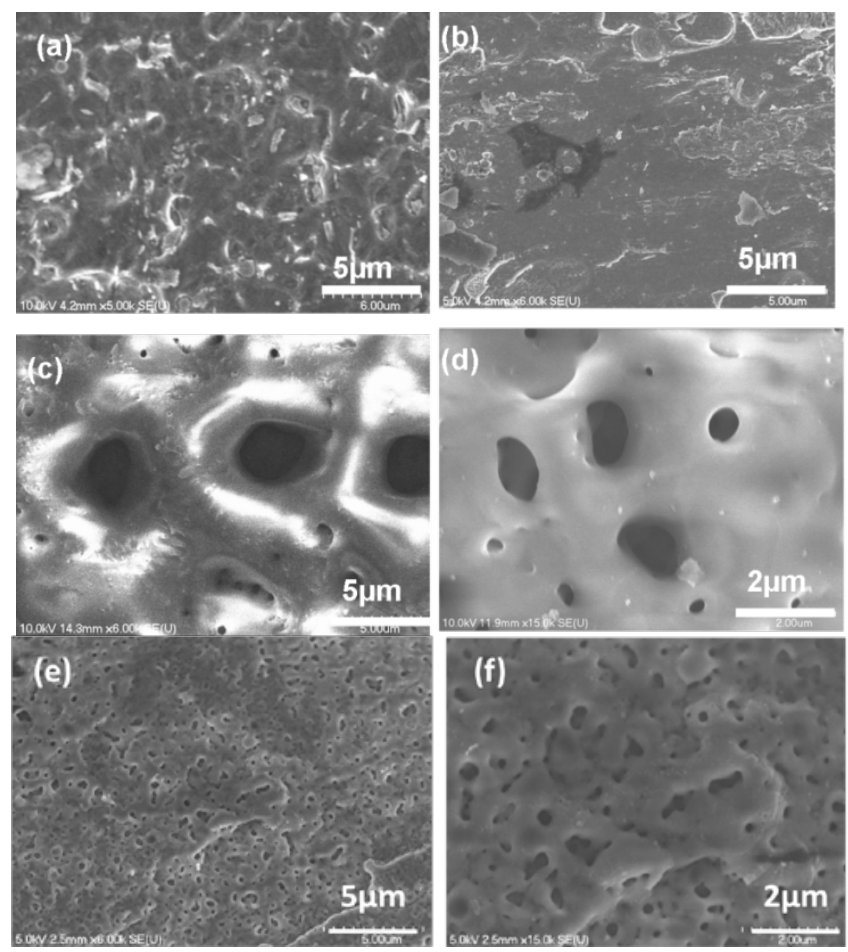

Figure 2.2. SEM micrographs of Ti-alloy anodized in $\mathrm{H}_{3} \mathrm{PO}_{4}$ and $\mathrm{HF}$ solution. (a and b) no porous structure in $50 \mathrm{~V}$ and $100 \mathrm{~V}$ respectively; (c and d) a porous structure of 3 micron and 1 micron size was formed at 150 and $200 \mathrm{~V}$ respectively;(e and f) a porous structure of $500 \mathrm{~nm}$. Scale bare $(\mathrm{a}, \mathrm{b}, \mathrm{c}, \mathrm{e})$ is $5 \mu \mathrm{m}$, and $(\mathrm{d}, \mathrm{f})$ is $2 \mu \mathrm{m}$.

\subsubsection{The Effect of HF Concentration}

This set of experiments was conducted to study the effect of electrolyte concentration of HF on the porous structure of Ti rod samples. The weight percent of HF was reduced from 1 to $0.5 \mathrm{wt} \%$. The concentration of phosphoric acid $\left(\mathrm{H}_{3} \mathrm{PO}_{4}\right)$ in the electrolyte solution was kept at the constant value of $1 \mathrm{M}$. The process was carried out for $10 \mathrm{~min}$ at 75,100 and $125 \mathrm{~V}$. Overall, the average pore size of $250 \mathrm{~nm}$ was observed (Figure 2.3). For the next set of experiments the HF concentration of $1 \mathrm{wt} \%$ was kept constant and the concentration of $\mathrm{H}_{3} \mathrm{PO}_{4}$ was increased up to $2 \mathrm{M}$. 


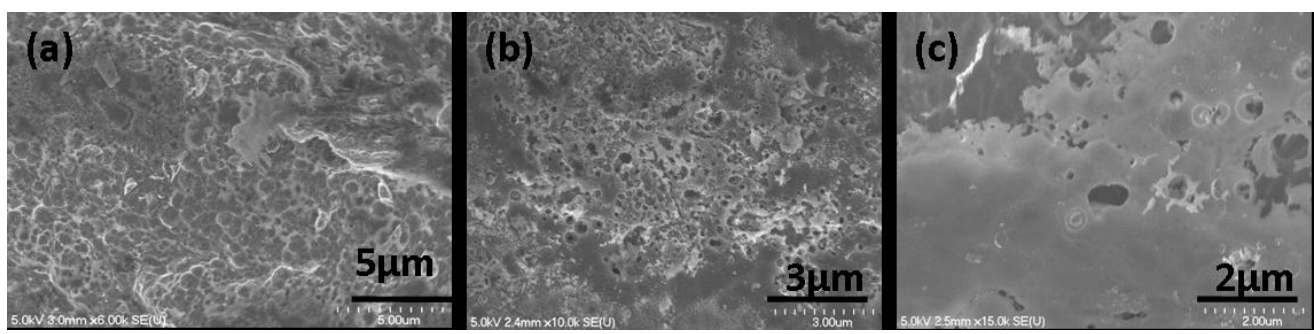

Figure 2.3. SEM images of Ti surfaces treated with HF (a) $75 \mathrm{~V}$, (b) $100 \mathrm{~V}$, (c) $125 \mathrm{~V}$. Scale bar (a) is $5 \mu \mathrm{m}$, (b) is $3 \mu \mathrm{m}$, and (c) is $2 \mu \mathrm{m}$.

\subsubsection{The Effect of $\mathrm{H}_{3} \mathrm{PO}_{4}$ Concentration}

These experiments were conducted to study the effect of $\mathrm{H}_{3} \mathrm{PO}_{4}$ electrolyte concentration on the porous structure of Ti rod samples. For this purpose, $1 \mathrm{wt} \%$ of $\mathrm{HF}$ was considered as the best concentration and $\mathrm{H}_{3} \mathrm{PO}_{4}$ concentration in the electrolyte solution was increased from $1 \mathrm{M}$ up to $2 \mathrm{M}$. The process was carried out for $5 \mathrm{~min}$ at 60 , 120,160 and $200 \mathrm{~V}$. The porous titanium structures were detectable at all voltage values. It was noticed that the pore size increased and the pore density decreased with the increase of voltage values. For $60,120,160$, and $200 \mathrm{~V}$ the average pore size of $0.15,0.3$, 0.42 , and 0.75 microns were observed respectively (Figure 2.4). 


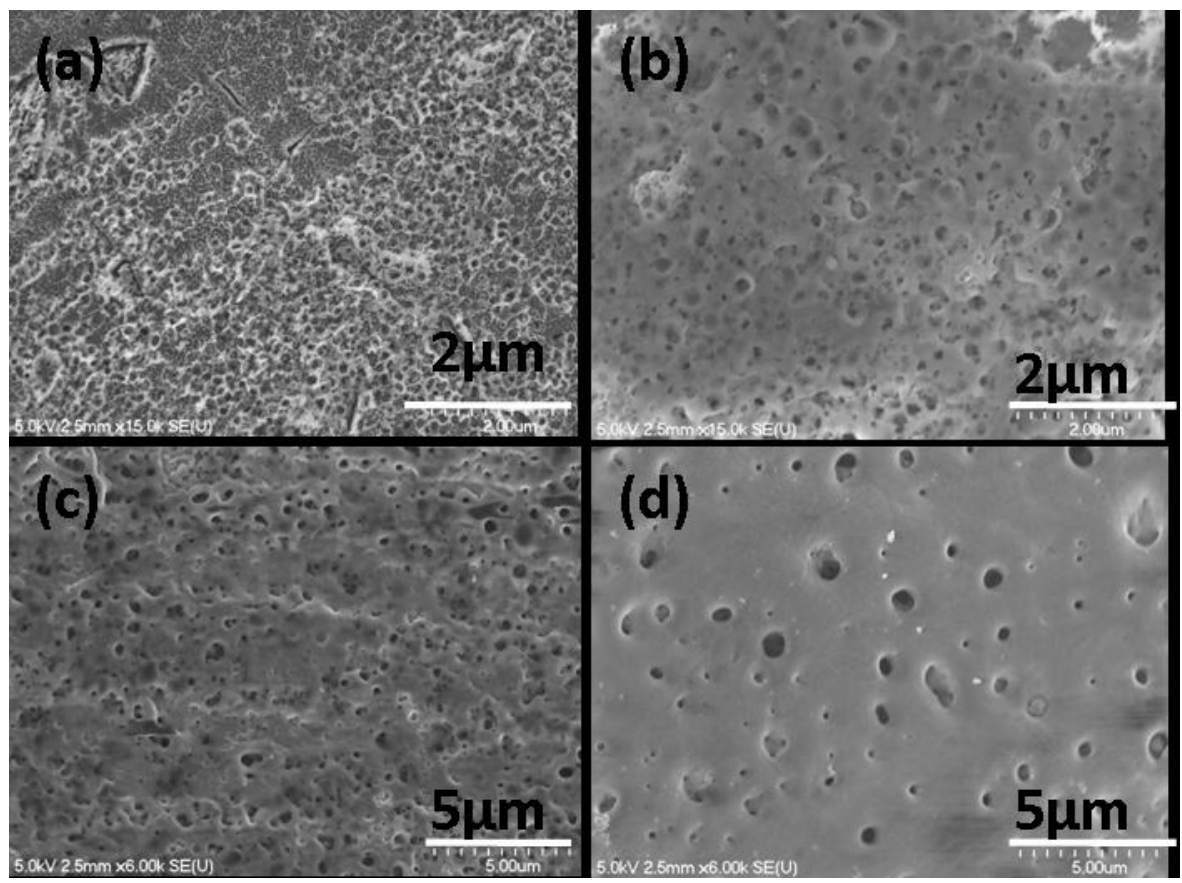

Figure 2.4. The effect of $\mathrm{H}_{3} \mathrm{PO}_{4}$ concentration at (a) $60 \mathrm{~V}$ and pore size range of 100-200 $\mathrm{nm}$; (b) $120 \mathrm{~V}$ and pore size range of 200-400 nm; (c) $160 \mathrm{~V}$ and pore size range of 350$500 \mathrm{~nm}$; (d) $200 \mathrm{~V}$ and pore size range of 400-1100 nm.

\subsubsection{Sandblasting Followed by Acid etching (SLA)}

Micro abrasive of 50-100 microns was used for the sandblasting process. This range was also used by Wenneberg et al. ${ }^{22}$ They demonstrated that the optimal surface roughness of particles of $75 \mu \mathrm{m}$ made the surface more resistant to torque failure and greater bone-to-metal contact than small $(25 \mu \mathrm{m})$ or coarse $(250 \mu \mathrm{m})$ particles. Acid etching of the SLA samples was carried out by a combination of sulphuric $\left(\mathrm{H}_{2} \mathrm{SO}_{4}\right)$ and hydrochloric $(\mathrm{HCl})$ acids. ${ }^{23}$ The titanium rod sample was first immersed in $\mathrm{H}_{2} \mathrm{SO}_{4}$ for a period of 72 hours. Next the sample was taken out from the acid, washed with distilled (DI) water, dried with nitrogen, and immersed into HCL for a period of 94 hours. Figure 2.5a shows the microstructure of surface oxide after the completion of the SLA process. 


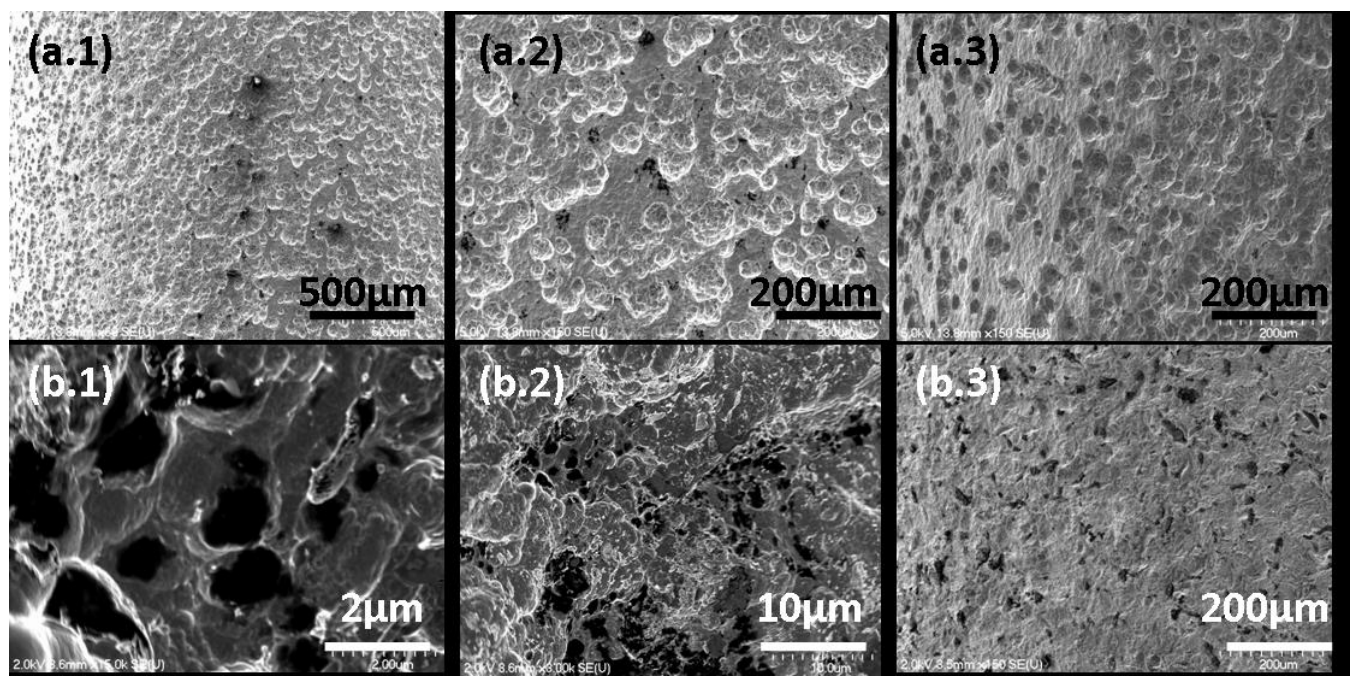

Figure 2.5. Surface structure of Ti oxide obtained after sandblasting and (a.1, a.2, a.3) acid etching (SLA) of the rods. (b.1, b.2, b.3) electro etching (SLE) of the rods. The structure shows micro-nano porosity and significant roughness. Scale bar (a.1) is $500 \mu \mathrm{m}$, (a.2, a.3, b.3) is $200 \mu \mathrm{m},($ b. 1 ) is $2 \mu \mathrm{m}$, and (b.2) is $10 \mu \mathrm{m}$.

\subsubsection{Sandblasting Followed by Electro Etching (SLE) Processes}

The electrochemical etching of the process was carried out under the conditions used

during the electrochemical etching series of experiments discussed in Sections 2.3.1.2 and 2.3.1.4.

\subsubsection{SLE Experiments with $1 \mathrm{wt} \% \mathrm{HF}+1 \mathrm{M} \mathrm{H}_{3} \mathrm{PO}_{4}$}

The process conditions were an aqueous electrolyte solution of $1 \mathrm{wt} \% \mathrm{HF}+1 \mathrm{M} \mathrm{H}_{3} \mathrm{PO}_{4}$, voltage value of $120 \mathrm{~V}$, and exposure time of 2 minutes. Figure $2.4 \mathrm{~b}$ shows the resulting etched surfaces. It can be seen that the size of the pores is on the average of $\sim 30$ microns. The nano pores were observed locally near and in between the micro pores. The average pore size of the nano pores was $\sim 600 \mathrm{~nm}$. 


\subsubsection{SLE Experiments with $1 \mathrm{wt} \% \mathrm{HF}+2 \mathrm{M} \mathrm{H}_{3} \mathrm{PO}_{4}$}

The electroetching experiments were conducted using an aqueous electrolyte solution of $1 \mathrm{wt} \% \mathrm{HF}+2 \mathrm{M} \mathrm{H}_{3} \mathrm{PO}_{4}$, and at $120 \mathrm{~V}, 160 \mathrm{~V}$, or $200 \mathrm{~V}$ and with exposure time of 5,3 , or 1 minute respectively. Figure 2.6 shows the resulting etched surfaces. It can be seen that the average size of pores is in the range of $\sim 30$ microns. The rod surface of these SLE samples contained two sets of structures. A roughened surface consisted of micro pores with average pore size of 30 microns. Nanopores in between the micropores with an average size of 300-750 $\mathrm{nm}$ were also detected.

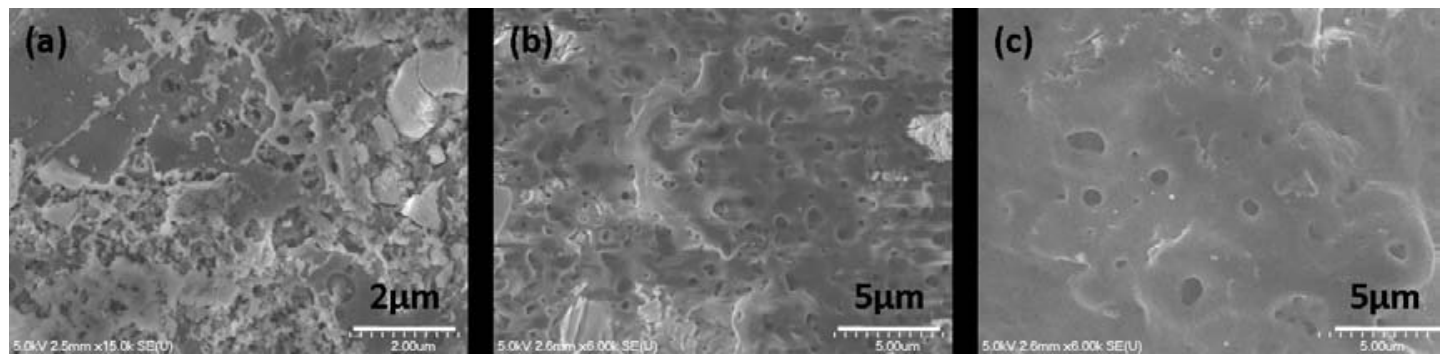

Figure 2.6. SEM images of sandblasted and electro etched (SLE) rods. The structure shows micro-nano porosity and significant roughness. Scale bar (a) is $2 \mu \mathrm{m}$ and $(b, c)$ is $5 \mu \mathrm{m}$.

\subsection{Discussion}

The results of the electrochemical etching process of machined and sandblasted samples indicated that the applied voltage is the main parameter that controlled the morphology of the porous structure in comparison to the exposure duration. The selective electrochemical etching (SEE) process resulted in a homogeneous distribution of nano porous structures. The sandblasted and acid-etched surfaces (SLA) were characterized as a combination of micro porosity and roughness. While the SLE, sand blasted and electroetched surfaces were observed as a combination of micro-nano porosity and micro roughness sandblasting, followed by the electrochemical etching process (SLE), indicated a new paradigm to develop novel bone and dental implants. In comparison between SLA and SLE processes, it was observed that the population of nanopores decreased while the average pore size increased with the increase of the voltage. 


\subsection{Conclusion}

The research summarizes a series of chemical-mechanical treatments to produce porous structures at the surface of Ti4Al6V. I conducted three methods of surface treatment including machined and selective electro-etched (SEE), sandblast and acid etched (SLA), and sandblasted and electroetched (SLE). The SEE process was conducted on machined Ti surfaces and resulted in small porous structures where the maximum pores were about $\sim 1-3$ microns. I found that the increase of applied voltage and concentration of $\mathrm{H}_{3} \mathrm{PO}_{4}$, increased the pore sizes at the surface of titanium. The SLA process was conducted on the sandblasted surfaces (roughened) and resulted in large pore sizes in the range of 10-30 microns. For the SLE process, in addition to the large porous structure of 10-30 microns, nanopores with an average size of $500 \mathrm{~nm}$ were observed. It is expected that the SLE process may be more desirable for implant application of titanium alloys due to the formation of such nanostructures. Others have shown that a nanophase structure in titanium improves osseointegration on the implants. Further research is necessary to study the biological response to the implant surface created by the SLE techniques. 


\title{
Chapter 3
}

\section{Synthesis of $\mathrm{TiO}_{2}$ Nanotubes}

\begin{abstract}
Orthopedic implants are often made of commercially pure titanium (cp-Ti, also referred to as Ti-grade 2) or titanium alloys for added mechanical properties. A common alloy is the aerospace alloy Ti6Al4V containing $6 \%$ aluminum and $4 \%$ vanadium (also referred to as Ti-grade 5). Implants are also made of less common alloys but are primarily titanium. Titanium is considered one of the most biocompatible metals because of its inertness and its stable outer oxide layer, $\mathrm{TiO}_{2}$.

To extend the functionality of the tubular materials by a new composition of oxides, I targeted the fabrication of binary oxide nanotubes and multicomponent oxide tubes using the Ti6A14V alloy. Tubular nanostructures can be electrochemically etched onto flat and non-flat surfaces and into foils of cp-Ti and Ti6Al4V alloy. This work used a cylindrical platinum mesh as the counter electrode approximately the same length as the foil and with a constant radial offset distance from the foil surface of approximately $2 \mathrm{~cm}$. To reduce the cost of the process, electrodes of materials other than platinum, and those that were stable in the ammonium fluoride and ethylene glycol electrolyte, were tested. In particular, copper was used. Tubular nanostructures were electrochemically etched with copper with no obvious morphological difference from those produced using a platinum electrode. Upon chemical analysis, it was determined that there were no detectable traces of copper within the sensitivity of the EDS analysis used.

This chapter focuses on the synthesis of nanotubes etched on the surface of Ti4Al6V. The nanotube arrays were synthesized by anodization in ethylene glycol electrolyte containing $0.2 \mathrm{wt} \% \mathrm{NH}_{4} \mathrm{~F}$ solution $(0.661 \mathrm{gr} \mathrm{NH} 4 \mathrm{~F}, 98 \mathrm{ml}$ Ethylene glycol, $2 \mathrm{ml}$ $\mathrm{H}_{2} \mathrm{O}$ ), and using copper as the cathode. The first part of this chapter relates to the mechanism of nanotube growth. The length of the nanotubes ranged from $200 \mathrm{~nm}$ to 6 $\mu \mathrm{m}$ by increasing the anodization voltage and time. The diameters of the nanotubes stay
\end{abstract}


almost the same with increased anodizing time but increase with increased voltage. Images captured from FE-SEM form the basis of the results.

\subsection{Introduction}

$\mathrm{TiO}_{2}$ is an n-type semiconductor and a photocatalyst, attracting attention from both fundamental and practical viewpoints. ${ }^{24}$ In recent years, many low dimensional $\mathrm{TiO}_{2}$ materials, such as nanowires, nanotubes, and nanorods have been successively synthesized. Among them, $\mathrm{TiO}_{2}$ nanotube structures are particularly interesting, partly because of their large specific surface areas and their tube-like structure. Gong et al. first produced titanium dioxide nanotube arrays with length up to $500 \mathrm{~nm}$ by electrochemical anodization in a HF aqueous electrolyte. ${ }^{25}$ Over recent years, the formation of titanium nanotube arrays has been produced by many other methods including template synthesis $^{26}$, layer-by-layer assembly ${ }^{27}$, and hydrothermal processes. ${ }^{28}$ Increased attention on titanium dioxide is due to the tubes' unique properties in optics, electronics ${ }^{29}$, photochemistry and biology ${ }^{30}$ and can be widely used as chemical substances, such as in areas of catalysis, gas-sensing, corrosion-resistance and solar energy materials.

In these applications, the control of morphology, particle size, particle size distribution, phase composition, and porosity of $\mathrm{TiO}_{2}$ is a vital factor in determining the properties of the final material. Several research groups ${ }^{31}$ have focused on the synthesis of nanotube with different diameter or length, ranging from $5 \mathrm{~nm}$ to several micrometers and a variety of shapes for photocatalysis and photoelectrode materials ${ }^{32}$, which are shown in table 3.1. 
Table 3.1 Nanotubes From Various Fabrication Method

\begin{tabular}{|c|c|c|c|c|}
\hline $\begin{array}{l}\text { Synthesis } \\
\text { Methods }\end{array}$ & Diameter & Length & Preparation Materials & Reference \\
\hline \multirow{3}{*}{$\begin{array}{l}\text { Anodization } \\
\text { Method }\end{array}$} & $65 \mathrm{~nm}$ & $250 \mathrm{~nm}$ & \multirow{3}{*}{$\begin{array}{l}\text { Either HF NaF or KF with } \\
\text { DMSO, EG, FA or NMF }\end{array}$} & 33 \\
\hline & $110 \mathrm{~nm}$ & $4.4 \mu \mathrm{m}$ & & $34,35,36$ \\
\hline & $150 \mathrm{~nm}$ & $134 \mu \mathrm{m}$ & & 37 \\
\hline \multirow{2}{*}{$\begin{array}{l}\text { Hydrothermal } \\
\text { Method }\end{array}$} & $9-10 \mathrm{~nm}$ & $122 \mathrm{~nm}$ & \multirow{2}{*}{$\begin{array}{l}\text { Precursor material with } \mathrm{TiO}_{2} \text { in } \\
\mathrm{NaOH} \text { solution }\end{array}$} & 38,39 \\
\hline & $20 \mathrm{~nm}$ & $1 \mu \mathrm{m}$ & & 40 \\
\hline \multirow{2}{*}{ So-gel } & $100 \mathrm{~nm}$ & $7 \mu \mathrm{m}$ & \multirow{2}{*}{$\begin{array}{l}\text { Template membrane dip into } \\
\qquad \mathrm{TiO}_{2} \text { gel }\end{array}$} & 41 \\
\hline & $120 \mathrm{~nm}$ & $1.5 \mu \mathrm{m}$ & & 42 \\
\hline $\begin{array}{l}\text { Layer by Layer } \\
\text { Assembly }\end{array}$ & $215 \mathrm{~nm}$ & $60 \mu \mathrm{m}$ & $\begin{array}{c}\text { Prepare } \mathrm{TiO}_{2} \text { and } \mathrm{TiO}_{2} \text { based } \\
\text { compound using Layer by } \\
\text { Layer strategy }\end{array}$ & 43 \\
\hline
\end{tabular}

From Table 3.1, it can be seen that different methods generated different sizes of nanotubes, including diameter and length. Even for the same method, different conditions also have diameter and length differences. However, from these four methods, the anodizaition method can produce the longest nanotubes up to $134 \mu \mathrm{m}$ and the hydrothermal method can make the shortest nanotubes up to $122 \mathrm{~nm}$ to $1 \mu \mathrm{m}$. For the diameter, the layer by layer assembly method can give the biggest porosity in the surface of nanotubes up to $215 \mathrm{~nm}$ and the hydrothermal method produces the smallest down to $20 \mathrm{~nm}$. Therefore, the anodization method is the most widely used because of its easy setup and short experiment time and the nanotubes from the hydrothermal method are short and small diameter.

Different methods will affect the physical properties of nanotubes. Various electrolytes containing fluoride ions also affect the length and diameter of the nanotubes, such as formamide ${ }^{44}$, ethylene glycol, and dimethyl sulfoxide. In this chapter, I use the 
electrochemical anodization method with the electrolyte component of $\mathrm{NH}_{4} \mathrm{~F}$ to quantify the length and diameter of nanotubes through changing the processing parameters of voltage and time.

\subsection{Mechanism of $\mathrm{TiO}_{2}$ Nanotube Formation}

Initially, titanium will react with the electrolyte to form a $\mathrm{TiO}_{2}$ layer on the surface. Then the $\mathrm{TiO}_{2}$ layer reacts with $\mathrm{NH}_{4} \mathrm{~F}$ when $\mathrm{F}^{-}$is the dominant ion in the system $^{45}$. After the anodization of titanium, the compact oxide begins to dissolve and dissolution takes place only in a selective area, which is etching. The etching rate is different at various locations of the oxide due to the differences in local stresses on the surface. This is called selective etching. The selective etching causes the surface to form porous structures. During the whole process, porous structures are formed by oxidation of titanium and subsequent oxide dissolution. These two processes can be summarized as two reactions ${ }^{46}$,

Oxidation: $\quad \mathrm{Ti}+4 \mathrm{H}_{2} \mathrm{O} \rightarrow \mathrm{TiO}_{2}+4 \mathrm{H}^{+}+4 \mathrm{e}$

Dissolution: $\mathrm{TiO}_{2}+6 \mathrm{~F}^{-}+4 \mathrm{H}^{+} \rightarrow\left[\mathrm{TiF}_{6}\right]^{2-}+2 \mathrm{H}_{2} \mathrm{O}$

Inside the pore channel there are two interfaces: solution-oxide and oxide-metal. The oxidation of titanium occurs at the oxide-metal interface near the pore bottom when the oxygen containing ions $\left(\mathrm{O}^{2-} / \mathrm{OH}^{-}\right)$transport from solution to the oxide layer, along the direction of the pore growth, which is described by reaction (1). At the same time, metal ions $\left(\mathrm{Ti}^{4+}\right)$ migrate from metal to the solution/oxide interface and dissolve into the solution, reaction (2). A electrical field enhances the migration of the metal ions and the latter process is called field-enhanced dissolution. As the electric field intensity at the pore bottom is much higher than that at the wall, titanium will be consumed at a higher rate near the bottom of the pore, allowing continuous increase of the pore depth. In contrast, for anodized titanium the final thickness of the porous oxide film does not increase with the anodizing time. 


\subsection{Experimental Procedure}

Titanium alloy foil (Ti6A14V, Grade 5) was used as the substrate for fabrication of the nanotube arrays. The foils were $1 \mathrm{~cm} \times 1 \mathrm{~cm}$ and were polished. The final polishing step was with a suspension of $60 \mu \mathrm{m}$ colloidal $\mathrm{SiO} 2$. Prior to etching, the samples were sequentially sonicated in acetone, isopropyl alcohol, and methanol, then rinsed with deionized water, and dried in an N2 stream. The experimental setup consisted of a twoelectrode arrangement, with $\mathrm{Cu}$ mesh as the counter-electrode during different sets of experiments. The spacing between the samples and the counter-electrode was approximately $25 \mathrm{~mm}$. Anodization was carried out at room temperature. The electrolyte was $0.2 \mathrm{wt} \% \mathrm{NH} 4 \mathrm{~F}$ solution in a plastic beaker. The voltage was supplied by a DC power supply and the current was measured by a digital multimeter. The anodization was at 20 , 40 and $60 \mathrm{~V}$ for three processing times of 4,8 and $16 \mathrm{~h}$ at room temperature. After completing the anodization experiments, the titanium foils were rinsed with deionized water and dried in atmosphere for further characterization. The surface structures were observed by a FE-SEM operating at $10 \mathrm{KeV}$. The distribution of nanotube diameter was calculated by image analysis. Energy dispersive spectroscopy (EDS) was used to collect chemical information of individual nanotubes and to confirm alloy properties.

\subsection{Results and Discussion}

\subsubsection{Effect of Driving Voltage on Anodization Kinetics}

Fig. 3.1 shows how the current density changes with time at 20, 40 and $60 \mathrm{~V}$ in the solution of $0.2 \mathrm{wt} \% \mathrm{NH}_{4} \mathrm{~F}$. When the voltage was applied to the system, there was a sudden current passing through the surface of the titanium foils. That initial current drop is probably due to the formation of a compact oxide film at the beginning stage which elevated the resistance and reduced the current densities. After that, the forming layer reacted with titanium to form the oxygen compound which was titanium dioxide on the surface. Because of the solubility of the titanium oxide in HF-containing solutions, there 
will be competition between the growing pores, and the current density decreases until a stable, ordered pore growth has been established.

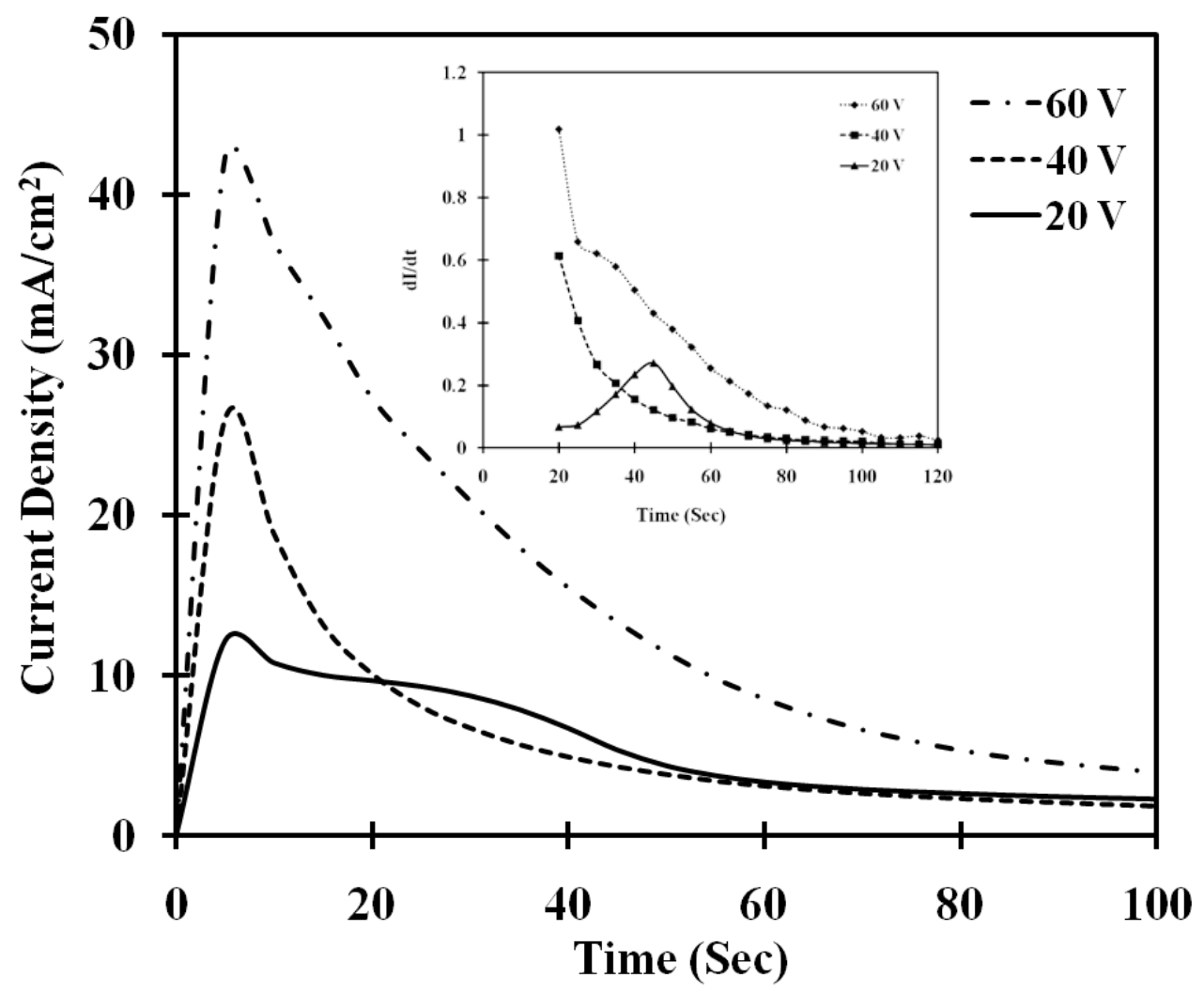

Figure 3.1. I-T curves for 20,40 , and $60 \mathrm{~V}$ during anodizing process in $0.2 \mathrm{wt} \% \mathrm{NH}_{4} \mathrm{~F}$ solution.

The maximum current increased by increasing the applied voltage. Considering the fact that the total resistance of the system remains the same in all three cases (no change in electrolyte and electrodes), the increasing maximum current means that the flow of electrons increased. The rate of oxidation reaction (reaction 1) increased by increasing the applied voltage. The high currents at $60 \mathrm{~V}$ and $40 \mathrm{~V}$ are associated with a higher rate of current decrease in comparison to the $20 \mathrm{~V}$ case. This is because the increase in reaction 1 means a faster growth of the oxide layer associated with increasing resistance and therefore the conductivity of the system decreases (inset Fig. 3.1). In the 40 and $60 \mathrm{~V}$ cases, the value of $\mathrm{dI} / \mathrm{dt}$ keeps decreasing because the oxide layers that formed from 
oxidation process increase the resistance of the system. This results in decreasing current. That is why the peak starts to increase then decrease during 20 to $60 \mathrm{sec}$ in $20 \mathrm{~V}$ case.

\subsubsection{Effect of Anodization Time on the Diameter of Nanotubes}

Different anodization time will lead to different nanotube diameters at the surface of titanium. Fig 3.2(a)-(d) show the images and the corresponding diameter analysis for samples after 4, 8 and $16 \mathrm{hrs}$ in the $0.2 \mathrm{wt} \% \mathrm{NH}_{4} \mathrm{~F}$ at $40 \mathrm{~V}$.

(a)

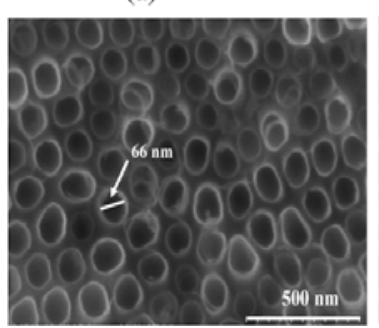

(b)

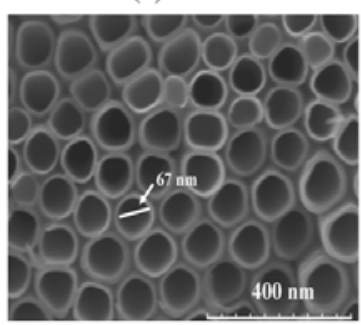

(c)

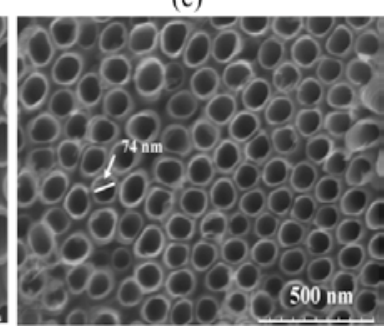

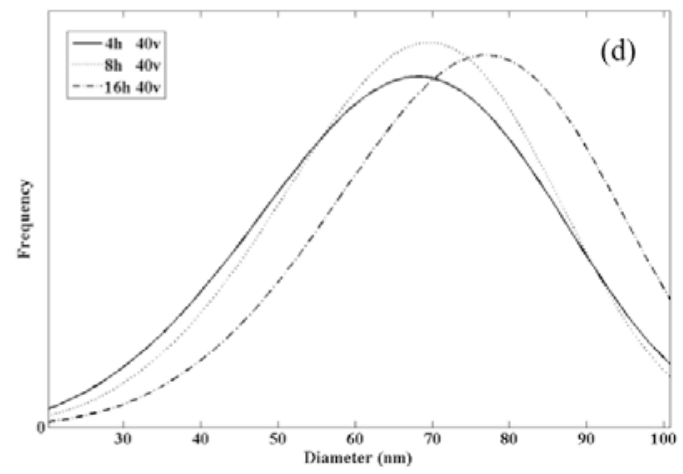

Figure 3.2. FE-SEM images of the surface structure obtained and the calculation of diameter distribution at $40 \mathrm{~V}$ in $0.2 \mathrm{wt} \% \mathrm{NH}_{4} \mathrm{~F}$ at different times (a) 4, (b) 8, and (c) 16 hrs and (d) diameter distribution with the average of 66, 67 and $74 \mathrm{~nm}$ for 4, 8 and 16 hours under $40 \mathrm{~V}$.

Fig. 3.2(a-c) shows the porous structure that formed in the surface of the substrate. The titanium foils were etched to individual tube structures. By measuring each inner diameter, the results are plotted in Fig. 3.2(d). In Fig. 3.2(d), the average diameter of nanotubes for $4 \mathrm{hrs}$ was found to be $66 \mathrm{~nm}$. As the time increased to 8 and $16 \mathrm{hrs}$, the average diameters increased to 67 and $74 \mathrm{~nm}$, respectively. There is not much difference in the diameter among the three samples anodized at the same voltage. Even though 
the time increased, the diameter stayed almost the same. This effect of time on diameter can be explained by the mechanism of tube formation. Under an applied voltage, the electric field assisted anodization of titanium will happen in the metal/oxide interface. After that, the dissolution process occurs which is an oxide/dissolution process. The diameter forms at the beginning of dissolution process and the reaction rate is very fast. It is only affected by the power of the electric field, which is voltage here. Combining the mechanism and results, I believe that time will not affect the oxidation part. Therefore, I don't expect any change in diameter, the effect of time on length will be discussed in the next section.

\subsubsection{Effect of Voltage on the Diameter of Nanotubes}

Fig. 3.3 shows the nanotube diameters at anodization voltages of 20,40 , and $60 \mathrm{~V}$ for $16 \mathrm{hrs}$. With the image from Fig. 3.2(c), a comparison with three different voltages under constant time can be made.
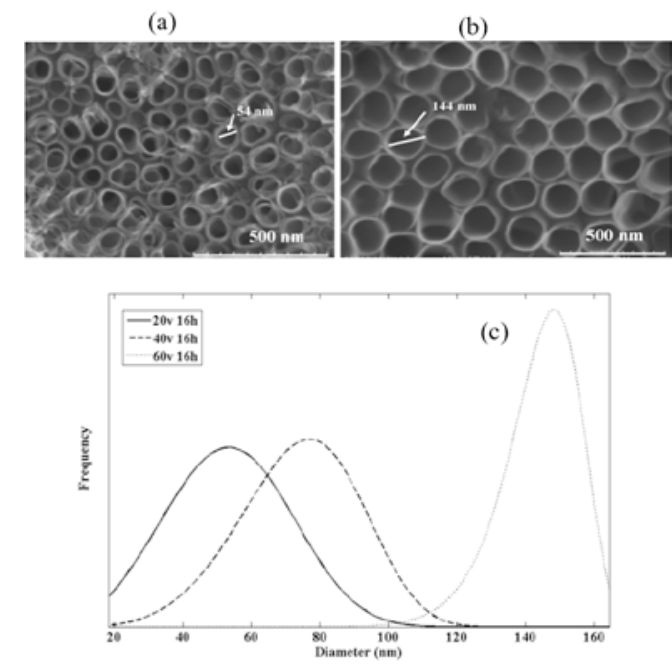

Figure 3.3. FE-SEM images of the nanotubes at 16 hours in $0.2 \mathrm{wt} \% \mathrm{NH}_{4} \mathrm{~F}$ at (a) $20 \mathrm{~V}$, and (b) $60 \mathrm{~V}$. (c) Diameter distribution of nanotubes at 20, 40 and $60 \mathrm{~V}$ for 16 hours. The average diameter is 54,74 and $144 \mathrm{~nm}$ for 20,40 , and $60 \mathrm{~V}$, respectively. 
It is shown in Fig. 3.3(a) that at $20 \mathrm{~V}$ the average diameter of the nanotubes was $54 \mathrm{~nm}$ as shown in Fig. 3.3(c). When the voltage was increased to $40 \mathrm{~V}$, the diameter also increased to $74 \mathrm{~nm}$ in Fig. 3.2(c). With further increase to $60 \mathrm{~V}$ in Fig. 3.3(b), the diameter was $144 \mathrm{~nm}$, which is almost double that of $40 \mathrm{~V}$. As discussed above, diameter formation is an etching process which happens in the oxide/solution interface. The voltage is the main factor affecting reaction speed. Higher voltage will supply more current that will make the electrical field stronger that will directly speed up the migration of metal ions. More and more titanium is involved in the dissolution process, which results in a large area formed in the surface.

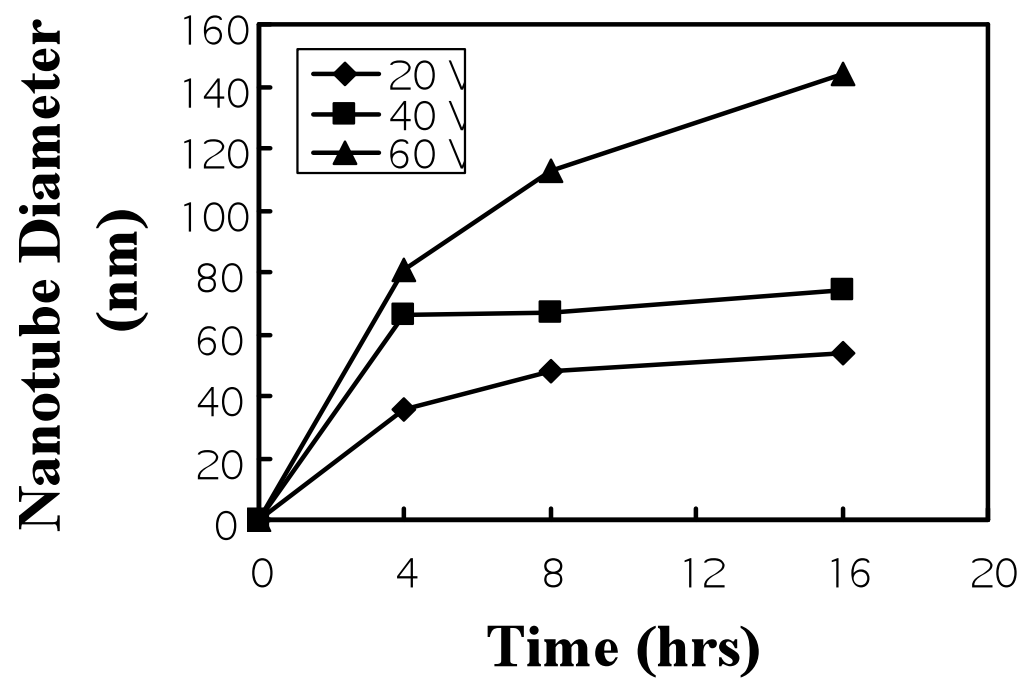

Figure 3.4. At the voltages of 20,40 and $60 \mathrm{~V}$, the inner diameter values are shown at anodization times of 4,8 and $16 \mathrm{hrs}$.

\subsubsection{Effect of Time on the Length of Nanotubes}

Figure 3.5 shows images of the nanotubes anodized at constant voltage of $60 \mathrm{~V}$ for 4, 8 and 16 hrs. From the cross section view in Fig. 3.5(a), the nanotube is already formed and all the nanotubes bundle together. It can be seen that some of the nanotubes were broken and twisted during the growth process. A nanotube with length of $5493 \mathrm{~nm}$ is shown in Fig. 3.5(a). When the time increased from 4 to $8 \mathrm{hrs}$, the same structure also happened and the length increased from $5493 \mathrm{~nm}$ to $6754 \mathrm{~nm}$ shown in Fig. 3.5(b). 
With $16 \mathrm{hrs}$ in (c), the length was up to $7000 \mathrm{~nm}$. As discussed above, the titanium metal will be consumed at a high rate near the bottom of the pore, allowing continuous growth of the pore depth, which is the nanotube length. The nanotube grows by etching deeper into the surface. From the I-T curves (Figure 3.1), one can see that the reaction rate stays in equilibrium for very long times apparently meaning the growth process will continue at a nearly constant speed. Thus, increasing anodization time will increase the length of the nanotubes.

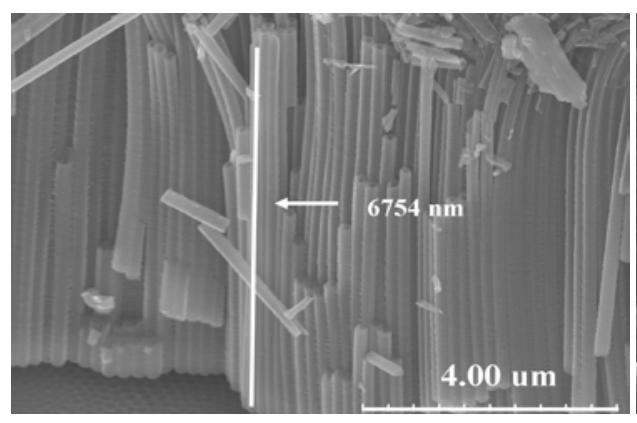

(a)

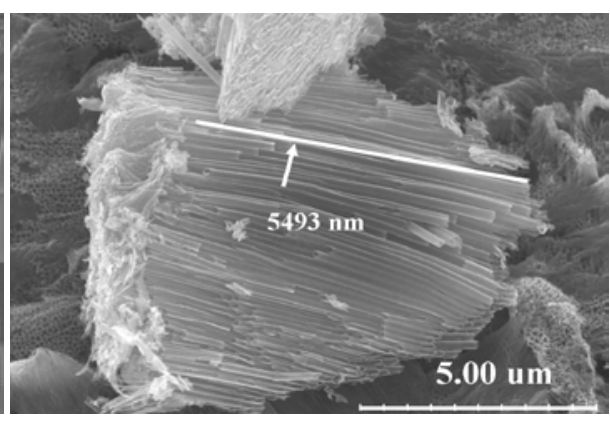

(b)

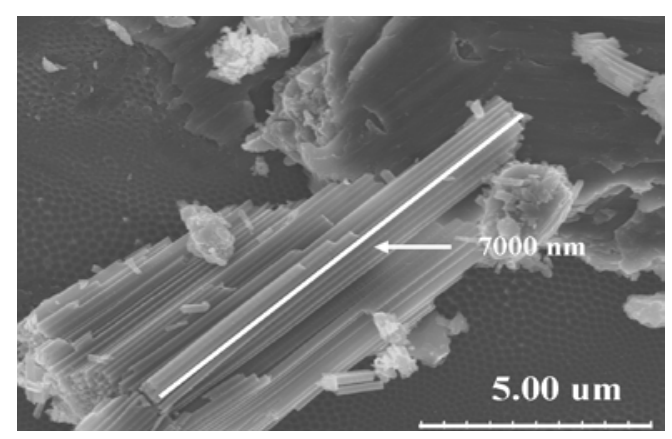

(c)

Figure 3.5. The microstructure nanotube obtained in $0.2 \mathrm{wt} \% \mathrm{NH}_{4} \mathrm{~F}$ at a voltage of $60 \mathrm{~V}$ and at (a) 4, (b) 8, and (c) 16 hrs.

\subsubsection{The Effect of Voltage on the Length of Nanotubes}

The SEM images of Fig. 3.6 captured the etching condition of 16 hours at 20 and 40 V. Combined with the image of Fig. 3.5(c), the results show the variation of produced nanotube morphologies at different voltages but at the same anodization time. From Fig. 3.6(a), the shape of the nanotube was formed but not completely. It looks like the strength 
of the nanotubes could be weak. Fig. 3.6(b) and Fig. 3.5(c) show the completely formed nanotubes. The length shown in the figure is $1388 \mathrm{~nm}$ at $16 \mathrm{hrs}$ at $20 \mathrm{~V}$. Compared to the images of Fig. 3.6(b) and Fig. 3.5(c), the length increased up to $6114 \mathrm{~nm}$ for $40 \mathrm{~V}$ and $7000 \mathrm{~nm}$ for $60 \mathrm{~V}$. From Fig. 3.7, it is seen that the voltage and time both have significant affect on growing nanotubes. The increase of time or voltage can increase the length of the nanotubes.

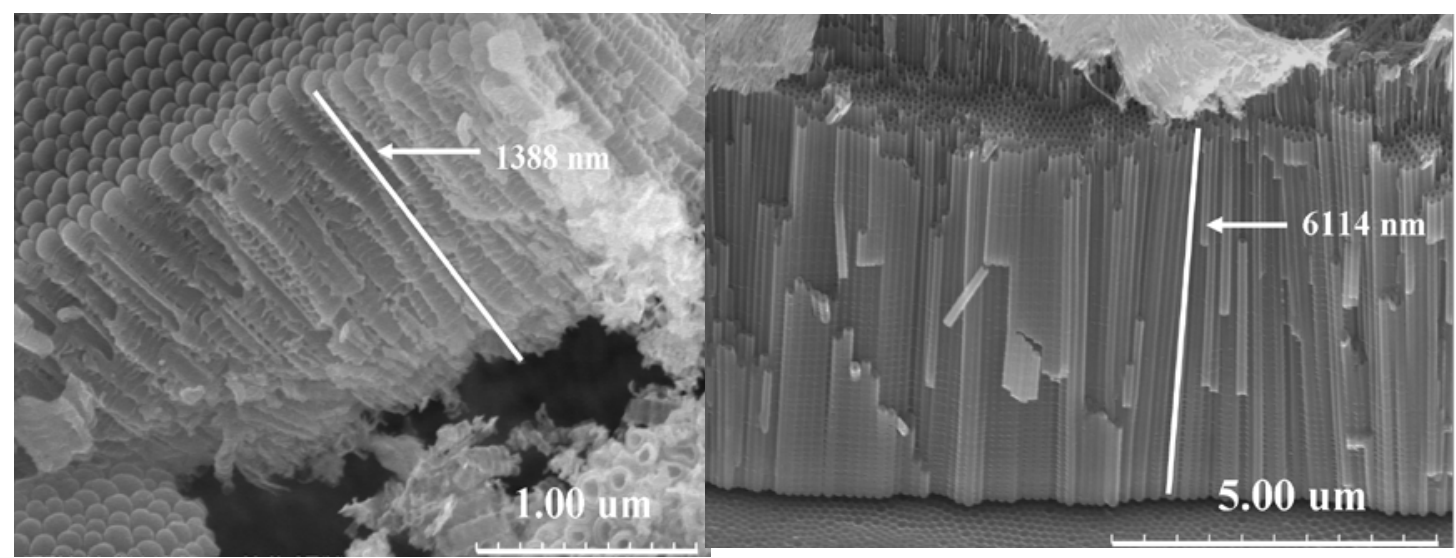

(a)

(b)

Figure 3.6. FE-SEM images of the microstructure obtained after $16 \mathrm{hrs}$ in $0.2 \mathrm{wt} \% \mathrm{NH}_{4} \mathrm{~F}$ at (a) 20 , and (b) $40 \mathrm{~V}$.

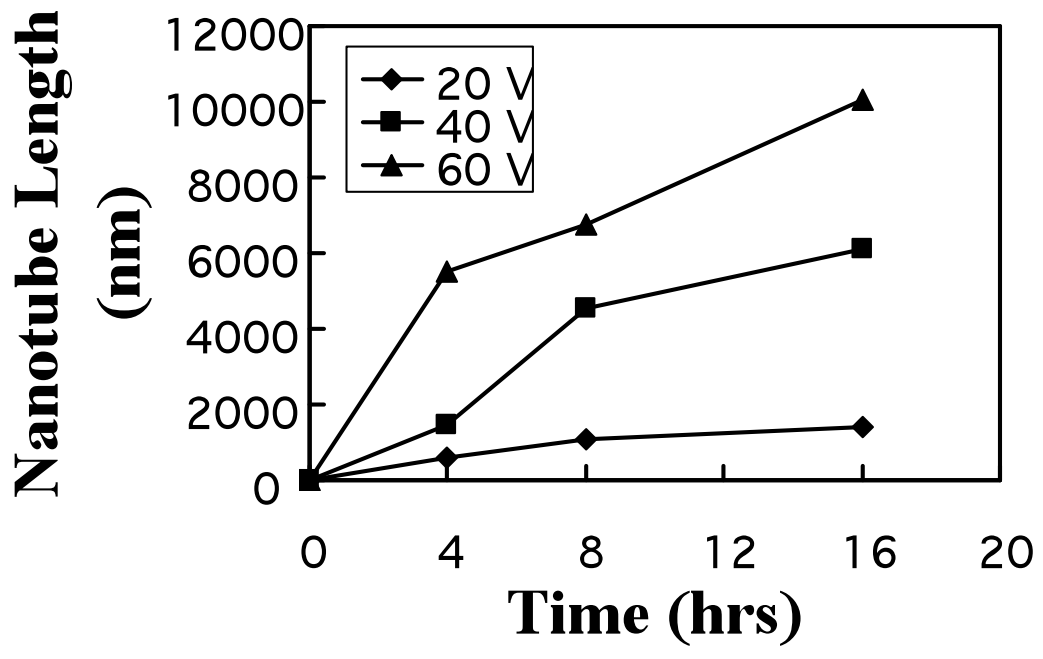

Figure 3.7. The nanotube lengths from 20,40 and $60 \mathrm{~V}$ at 4,8 and $16 \mathrm{hrs}$. 


\subsection{Chemistry of $\mathrm{TiO}_{2}$ Nanotubes}

The energy-dispersive X-ray spectroscopy (EDS or EDX) results shown in Figure 3.8 indicated the chemical structure of the alloyed $\mathrm{TiO}_{2}$ nanotubes, with a chemical ratio of Ti:O:Al: $\mathrm{V}=69.64: 21.02: 6.15: 3.19$. Upon chemical analysis, it was determined that there were no detectable traces of copper from the cathode, within the sensitivity of the EDS measurements.

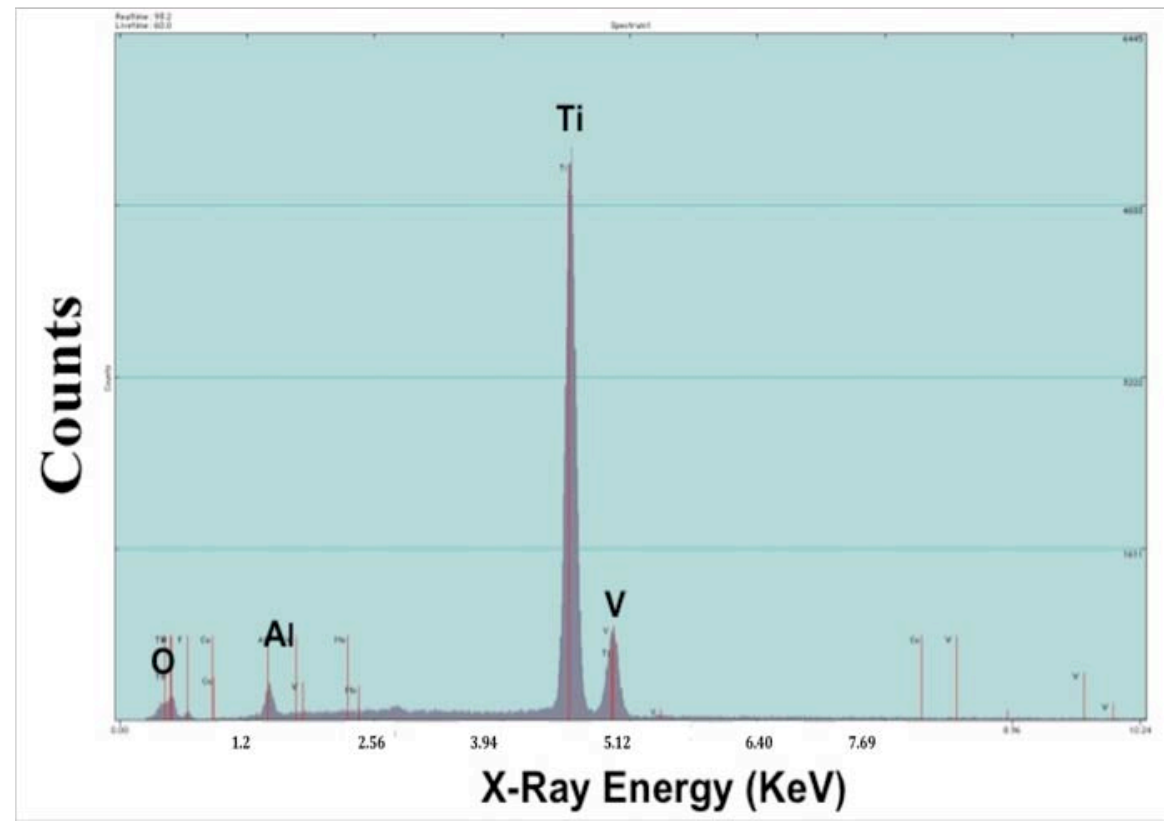

Figure 3.8. EDS analysis of a single alloyed (Ti6Al4V) nanotube indicating the Ti, Al,V, and $\mathrm{O}$ peaks, The EDS analysis confirmd that there was not any detectable extra element introduced to the rod surface during the formation of the nanotubes.

\subsection{Conclusion}

The present work inve stigated the diameter and length changes by controlling the time and voltage of anodizing Ti6Al4V foil to get nanotubes using $\mathrm{NH}_{4} \mathrm{~F}$ solution. I observed that the increase of anodization and time will increase the length and diameter of nanotubes. Based on the potential application of $\mathrm{TiO}_{2}$ nanotube in medical applications, it is necessary to do more work on controlling the size and length, which will get us better efficiency or quality of the materials. 


\title{
Chapter 4
}

\section{Direct Compressive Measurements of Individual Titanium Dioxide Nanotubes}

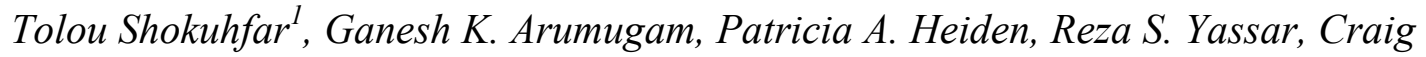 \\ Friedrich
}

ACS Nano, 3 (2009), 3098-3102

\begin{abstract}
The mechanical compressive properties of individual thin-wall and thick-wall $\mathrm{TiO}_{2}$ nanotubes were directly measured for the first time. Nanotubes with outside diameters of 75 and $110 \mathrm{~nm}$ and wall thicknesses of $5 \mathrm{~nm}$ and $15 \mathrm{~nm}$, respectively, were axially compressed inside a $400 \mathrm{keV}$ high-resolution transmission electron microscope (TEM) using a new fully integrated TEM-atomic force microscope (AFM) piezo-driven fixture for continuous recording of the force-displacement curves. Individual nanotubes were directly subjected to compressive loading. We found that the Young's modulus of titanium dioxide nanotubes depended on the diameter and wall thickness of the nanotube and is in the range of $23-44 \mathrm{GPa}$. The thin-wall nanotubes collapsed at $\sim 1.0-1.2 \mu \mathrm{N}$ during axial compression.
\end{abstract}

KEYWORDS: Titanium oxide nanotubes, Mechanical Properties, In-situ TEM

\subsection{Introduction}

$\mathrm{TiO}_{2}$ is a highly functional material with a wide range of applications that are based on its semiconductive and biocompatible nature. The semiconductive nature makes $\mathrm{TiO}_{2}$

1 As the first author of this publication I have performed all the experimental studies as well as results, discussion and conclusion. 
suitable for water-splitting, photocatalysis and for self-cleaning applications. ${ }^{47,48,49}$ Biocompatibility is highly important in biology and medicine, where the $\mathrm{TiO}_{2}$ layers on Ti and Ti alloys are in direct contact with biological tissue in hip or dental implants ${ }^{50}$ For several applications, a nanostructured layer with a high surface area, possibly a uniform distribution, and excellent charge-transfer properties is beneficial for good performance.

Gong and co-workers reported the synthesis of $\mathrm{TiO}_{2}$ nanotube arrays using an aqueous HF based electrolyte. ${ }^{51}$ The $\mathrm{pH}$ of $\mathrm{F}^{-}$ion containing electrolytes was controlled to form nanotubes up to a few micrometers in length. They reported that the tube properties varied dramatically with the electrolyte chemistry in which they were formed. For instance, close-packed hexagonal tubes were formed when using an ethylene glycol electrolyte. ${ }^{52}$ Removal of the tube "plug" by an acid rinse enabled fabrication of freestanding, self-supportive membranes that can be used for biofiltration..$^{33,54}$ In contrast, tubes formed from dimethyl sulfoxide (DMSO) electrolytes had weak tube-to-tube binding. Further studies focused on precise control and extension of the nanotube morphology ${ }^{55}$, length and pore size ${ }^{56}$, and wall thickness. ${ }^{57}$ Various doping strategies have been pursued including the use of an organic anodization bath, and incorporation of anionic species during the anodization process. ${ }^{58}$

To our knowledge, direct measurements of the compressive mechanical properties of individual $\mathrm{TiO}_{2}$ nanotubes with in-situ TEM have not been reported. Only very recently, Crawford et al. used nanoindentation to measure the Young's modulus of $\mathrm{TiO}_{2}$ nanotube films deposited on $\mathrm{Ti}$ substrates. ${ }^{59}$ The authors reported a significant contribution from the substrate on the force measurements, and used the role of mixture for composite materials (weighted sum of modulus-volume fractions of the constituents) to estimate the Young's modulus of the nanotube films. In the present work we performed direct compressive force measurements of titanium dioxide nanotubes inside a $400 \mathrm{keV}$ high-resolution field-emission transmission electron microscope eliminating the prior approximation. A novel integrated AFM-TEM holder equipped with a MEMS force sensor enabled the in-situ and direct quantitative measurements of the nanotube compressive characteristics. The mechanical tests in the TEM allowed us to not only evaluate the compressive stresses and estimate the elastic modulus of individual 
$\mathrm{TiO}_{2}$ nanotubes, but also to monitor and dynamically record the tube structural changes during loading.

\subsection{Experimental Procedure}

Titanium foils ( $250 \mu \mathrm{m}$ thick, purity $\sim 99.7 \%$, Sigma-Aldrich) were ultrasonically cleaned in acetone and ethanol. The foils were anodized in a two-electrode electrochemical cell in an electrolyte containing dimethyl Sulfoxide (DMSO) (99.6\%, Aldrich) and hydrofluoric acid (HF, 48\% aqueous solution, JT Baker). The anodization voltage, HF concentration in DMSO, and duration of anodization were $60 \mathrm{~V}, 2 \mathrm{wt} \%$, and 40 hrs, respectively. Platinum foil was used as the counter electrode. The distance between the two electrodes was maintained at $2 \mathrm{~cm}$. All experiments were conducted at about $25{ }^{\circ} \mathrm{C}$. After anodization, samples were washed with deionized (DI) water and ultrasonicated to remove surface debris consisting of precipitate that fell out of solution onto the sample. The nanotube array morphology was imaged using a field emission scanning electron microscope (FESEM, Hitachi S-4700). The nanotubes where then annealed in ambient oxygen at $450{ }^{\circ} \mathrm{C}$ for $3 \mathrm{hr}$.
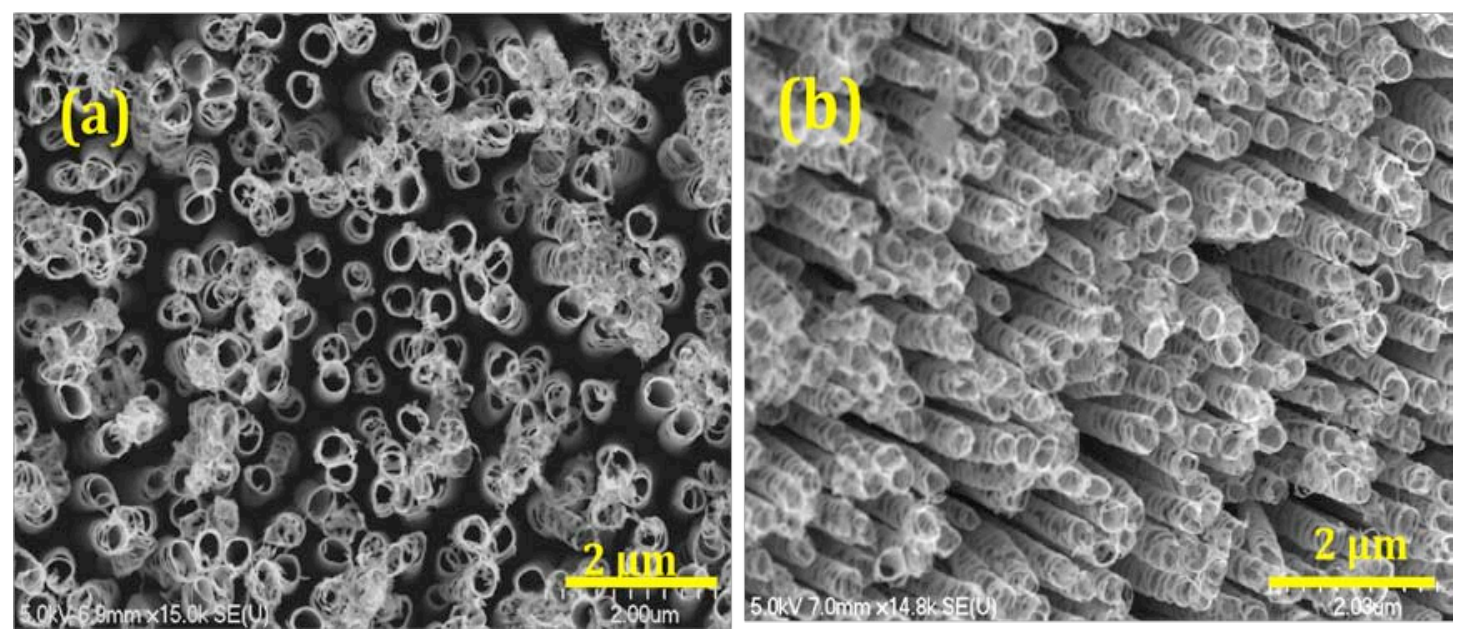

Figure 4.1. FESEM images of a $\mathrm{TiO}_{2}$ nanotube array sample etched with a $2.0 \% \mathrm{HF}$ -

DMSO electrolyte for $40 \mathrm{hrs}$ at $60 \mathrm{~V}$. 


\subsection{Results and Discussions}

The anodization resulted in vertically oriented $\mathrm{TiO}_{2}$ nanotube arrays as shown in Figure 4.1. Nanotubes had lengths between 7-10 microns and inner diameters ranging from $60-130 \mathrm{~nm}$. The tubes were easily dispersed enabling their use. This allowed us to directly measure the properties of an individual tube and to characterize the compressive behavior.

With respect to the experimental verification of mechanical properties of $\mathrm{TiO}_{2}$ nanotubes, no direct measurements appear to have been reported. Crawford et al. used nanoindentation to probe the mechanical response of $\mathrm{TiO}_{2}$ nanotube films on a $\mathrm{Ti}$ substrate. They proposed that the regions immediately under the indenter tip were subjected to densification, while those at the sides of the indenter were under shear stress that induced both densification and wear of the nanotubes. No direct measurement of force-displacement on a single nanotube was reported, however the authors did report progressively higher values of the Young's modulus for thinner films of $\mathrm{TiO}_{2}$ nanotubes consistent with the increased influence of the underlying Ti substrate.

In the present work we performed direct force-displacement measurements during compressive loading of high purity, well-structured individual $\mathrm{TiO}_{2}$ nanotubes inside a $400 \mathrm{keV}$ high-resolution JEOL TEM. The accuracy and capability of this technique for

direct measurements have been discussed in a number of recent publications ${ }^{60,61}$. For instance Golberg et al. measured the bending force and modulus of boron nitride nanotubes using an in-situ AFM-TEM technique. In our work, an AFM cantilever holder equipped with a MEMS force sensor enabled in-situ and direct measurements of the compressive properties of the nanotubes. The experimental setup is shown schematically in Figure 4.2. A silicon cantilever was attached to a fixed MEMS force sensor and a gold wire with a mounted $\mathrm{TiO}_{2}$ nanotube was placed on the piezo-movable side of the holder. Initially, the relative positions of the wire and cantilever were manually adjusted with tweezers under an optical microscope to reduce the gap between them. Finally, the $X, Y$, and $Z$ positions of the cantilever and individual $\mathrm{TiO}_{2}$ nanotube were adjusted with the precision piezo-driven manipulator of the gold wire. The nanotube motion and force acquisition parameters were controlled by software and electronics from Nanofactory 
Instruments $\mathrm{AB}$. This automatically coordinated the stages and controlled the $\mathrm{TiO}_{2}$ nanotube displacement and rate. Prior to nanotube compression, the sensor system was calibrated by indenting an Au wire, and the calibration coefficients were introduced into the sensor system software. During the experiments, we monitored and recorded the compressive force-displacement of the individual $\mathrm{TiO}_{2}$ nanotubes.

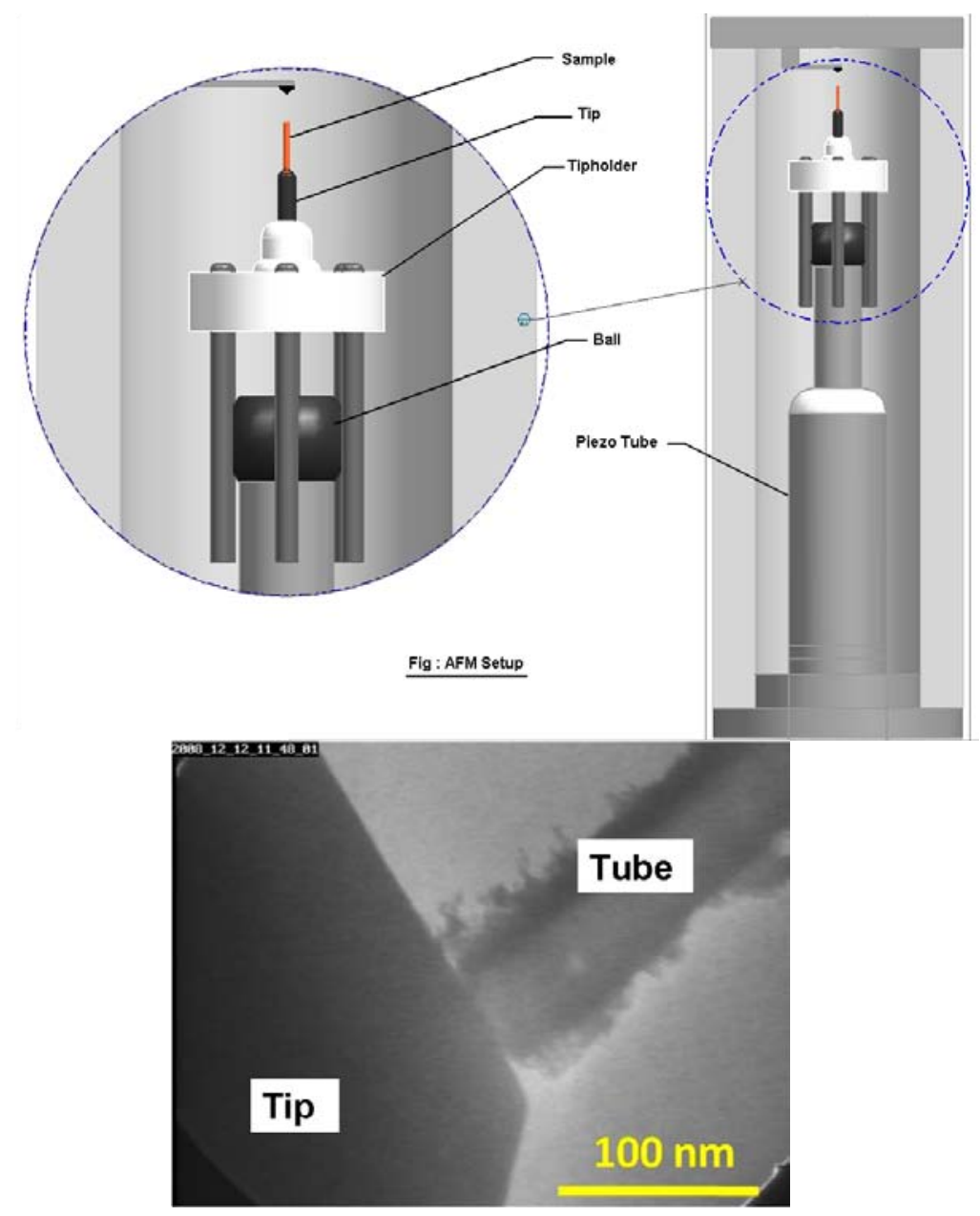

Figure 4.2. Schematic of the experimental setup within an AFM-TEM holder. The inset on the right shows a TEM view of a titanium dioxide nanotube being compressed between a sample wire and a silicon cantilever. The initial position of the tube against the cantilever may be precisely adjusted with piezo-driven displacement of the sample wire. 
Two representative $\mathrm{TiO}_{2}$ nanotubes were used for in-situ TEM measurements, which we refer to as "thin-wall" and "thick-wall". The thin-wall tube had external and internal nominal diameters of $\sim 75$ and $\sim 65 \mathrm{~nm}$. The thick-wall tube had external and internal nominal diameters of $\sim 110 \mathrm{~nm}$ and $\sim 80 \mathrm{~nm}$. The typical lengths of tubes prior to compression were $7-10 \mu \mathrm{m}$.
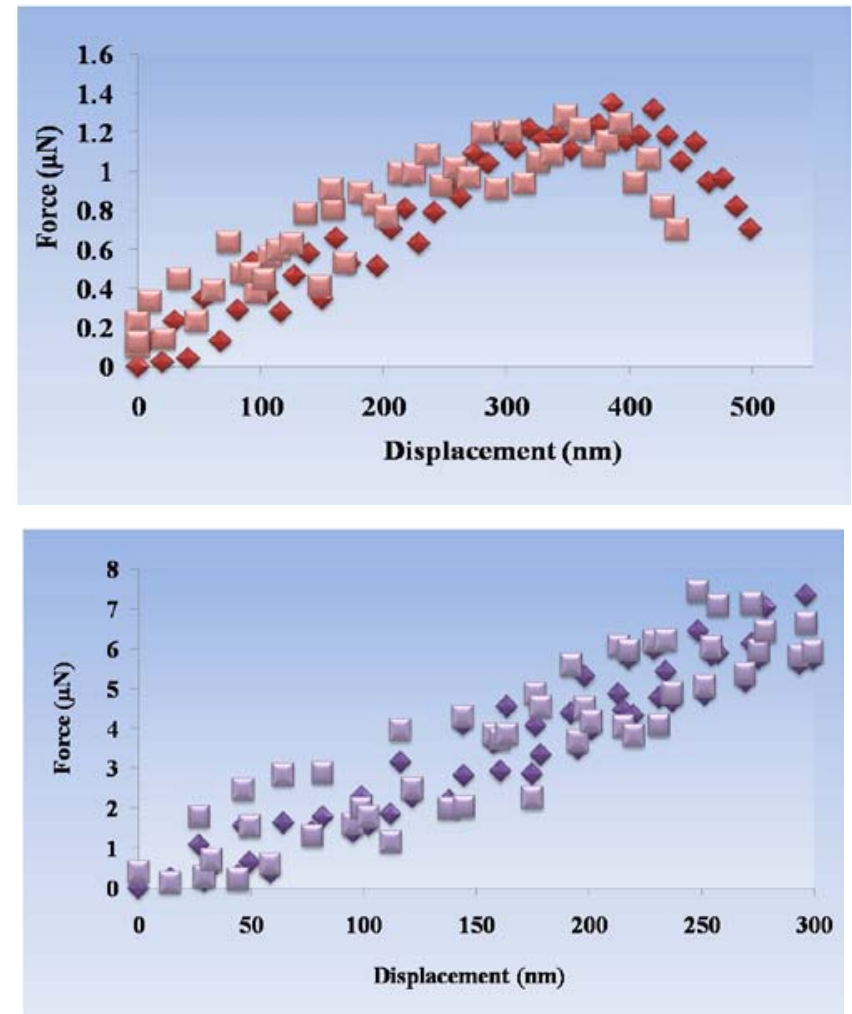

Figure 4.3. Force vs. displacement curves recorded for the thin-wall (top) and thick-wall (bottom) $\mathrm{TiO}_{2}$ nanotubes. The data from multiple experiments for each tube is overlaid in each plot with different color.

The typical force-displacement curves of the thin-wall and thick-wall $\mathrm{TiO}_{2}$ nanotubes during compression are shown in Figure 4.3. The compression tests were repeated for several nanotubes to ensure the reproducibility of the data as they are shown with different color in each plot. In general, for Figure $4.3 \mathrm{a}$ and $\mathrm{b}$, the maximum observed standard deviation were $0.25 \mu \mathrm{N}$ and $0.85 \mu \mathrm{N}$ respectively. The maximum applied forces were directly measured to be $\sim 1.2 \mu \mathrm{N}$ for the thin-wall tube and $\sim 7 \mu \mathrm{N}$ for the thick-wall tube. By measuring the $\mathrm{TiO}_{2}$ nanotube internal and external nominal 
diameters and length in the TEM, we were able to estimate the compressive stresses to be $1091 \mathrm{MPa}$ for the thin-wall tube and $1475 \mathrm{MPa}$ for the thick-wall tube. We refer to the nominal diameter because the cross-section dimensions of a tube were estimated by its two dimensional projection viewed in the TEM. To calculate the elastic modulus, we used the engineering stress and the engineering strain based on an average tube length of $8.5 \mu \mathrm{m}$. We obtained the elastic modulus of the nanotubes to be $23 \mathrm{GPa}$ and $44 \mathrm{GPa}$ for thin-wall and thick-wall tubes, respectively. The calculated elastic modulus data are in close agreement with the Young's modulus values reported by Crawford et al. who used Nanoindentation. ${ }^{59}$ Their reported values were in the range of 36-43 GPa. Our approach is different and provides direct force measurements on a single nanotube. Although the highest elastic modulus that we obtained is similar to that of Crawford et al, we found nanotubes with a lower Young's modulus $(23 \mathrm{GPa})$ than the values reported by these authors (36GPa).

Moreover, the in-situ TEM studies allowed us to correlate the load-displacement measurements with changes in the tubes' structure. During compressive loading, a phenomenon was observed that corresponds to the drop of force on the thin-wall tube shown in Figure 4.3. The correlation of load measurements with TEM images indicated that the thin-wall $\mathrm{TiO}_{2}$ nanotube exhibited a force-displacement response similar to yielding in a conventional ductile material. A TEM image of a thin-wall nanotube is shown in Figure 4.4 and the inset shows the collapsed zone which apparently caused the drop in compressive force. The collapse of thin-wall nanotubes could be due to localized deformation and failure originated by nanoscale defects. As seen in the TEM images, the nanotube surface contains irregularities and this may have caused stress concentration and failure.

The collapse of carbon nanotubes due to compressive loading has been predicted by atomistic simulation. Yokobson et al. observed that under axial compression, a carbon nanotube undergoes various atomistic mechanisms. ${ }^{62}$ Within the Hookean range of strain, and even at larger strains where the stress-strain relation deviates from linear, they did not see any bond switching or breaking in the nanotube. There were no indications of plastic behavior, i.e., the formation of dislocation-like defects and their motion 
within the strained nanotube body. At a certain critical level, which is natural to identify with the breaking strain, one or a few $\mathrm{C}-\mathrm{C}$ bonds break almost simultaneously, and the resulting 'hole' in the tube wall became a precursor of fracture. The atomic disorder propagated very quickly along the circumference of the tube. The strain, which was quite uniform along the tube before this threshold, now redistributed itself to form a largely distorted and unstable neck between the two relaxing nanotube segments.

Using the Euler formula, $\sigma_{c r}=\pi^{2} E I /\left(A L^{2}\right)$, where $\sigma_{c r}$ is the critical stress, $E$ is the elastic modulus, $I$ is the moment of inertia, $A$ is the column cross section, and $L$ is the length of the column, one might expect to observe buckling phenomenon rather than collapse by compression. However, certain conditions need to be met before applying the Euler formula to an axially stressed column. The Euler buckling equation is only valid for long and slender columns loaded axially in compression. What determines this long and slender criterion is the ratio of the effective length $(K L)$ of a column to the least radius of gyration, $r=(I / A)^{0.5}$, of its cross section. Considering a fixed end $(K=0.5)$ scenario, in the case of our $\mathrm{TiO}_{2}$ nanotubes, this ratio was calculated to be $\sim 120$. This value can be categorized in the intermediate range of slender ratios, and buckling failure can happen at higher slender ratios.

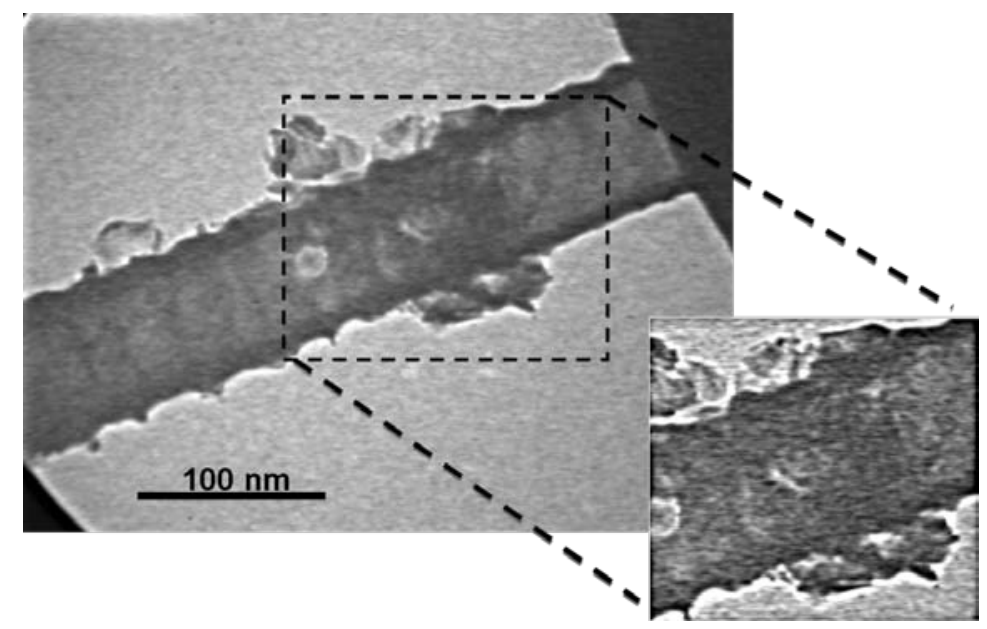

Figure 4.4. $\mathrm{A} \mathrm{TiO}_{2}$ nanotube fractured after application of maximum compressive load. The inset shows the collapse of the nanotube due to compressive load. 
The compression experiments inside the TEM allowed us to track the structural changes within an individual deformed nanotube. This is an appealing advantage of the present in-situ TEM technique over the AFM and SEM-based setups, which suffer from insufficient spatial resolution leaving unanswered deformation-related questions about elastic and/or plastic deformation. It is interesting to note that the Young's modulus monotonically decreases with the bulk $\mathrm{TiO}_{2}$, the thick-wall, and thin-wall nanotube dimensions.

\subsection{Conclusion}

Direct force measurements were performed during compressive deformation of individual titanium dioxide nanotubes using an AFM cantilever, piezo-driven holder inside a $400 \mathrm{keV}$ JEOL high-resolution field-emission transmission electron microscope. The measured forces ranged between $1.2 \mu \mathrm{N}$ and $7 \mu \mathrm{N}$. These forces, coupled with the observed $\mathrm{TiO}_{2}$ nanotube dimensions, correspond to compressive stresses up to $1564 \mathrm{MPa}$ and lead to a Young's modulus of $\mathrm{TiO}_{2}$ nanotubes in the range of $23 \mathrm{GPa}$ to $44 \mathrm{GPa}$. $\mathrm{By}$ taking advantage of the presumed changes in deformation mechanisms within nanocrystalline materials such as the titanium dioxide nanotubes reported here, it is possible that much higher specific strengths can be realized, and structural deformation at the nanoscale can be exploited to make advanced engineering materials.

\section{Acknowledgment}

The authors thank the Multi-Scale Technologies Institute and the Applied Chemical and Morphological Analysis Laboratory, Michigan Technological University for providing TEM support to conduct this research. 


\title{
Chapter 5
}

\subsection{A Study on the Modulation of the Electrical Transport by Mechanical Straining of Individual $\mathrm{TiO}_{2}$ Nanotube}

\author{
Anjana Asthana, Tolou Shokuhfar ${ }^{2}$, Qi. Gao, Patricia A Heiden, Craig Friedrich, Reza
}

S. Yassar

Applied Physics Letters, 97 (2010), 072107

\begin{abstract}
We report here, the deformation driven modulation of the electrical transport properties of an individual $\mathrm{TiO}_{2}$ nanotube via in-situ transmission electron microscopy (TEM). The current-voltage characteristics of each individual $\mathrm{TiO}_{2}$ nanotube revealed that under bending deformation within the elastic limit, the electrical conductivity of a $\mathrm{TiO}_{2}$ nanotube can be enhanced. Analysis based on a metal-semiconductor-metal model suggests that in-shell, surface defect-driven conduction modes and electron-phonon coupling effect are responsible for the modulated semiconducting behaviors.
\end{abstract}

Keywords: $\mathrm{TiO}_{2}$ Nanotube, Electrical properties, deformation, Anatase $\mathrm{TiO}_{2}$

\subsubsection{Introduction}

In recent years quasi one dimensional (1D) solid nanostructures have stimulated considerable interest for scientific research due to their importance for fundamental studies of the roles of dimensionality and size in their physical properties as well as their potential technological applications.

${ }^{2}$ I have contributed to this publication by brainstorming the idea and synthesizing the main material of characterization ( $\mathrm{TiO}_{2}$ nanotubes). 
Among the $1 \mathrm{D}$ oxide materials, titania $\left(\mathrm{TiO}_{2}\right)$ in various morphologies (tubes, wires, fibers, and rods) has attracted much attention from both fundamental and practical viewpoints. Their semiconducting behavior and large surface area have drawn considerable attention for potential applications in solar cells ${ }^{63}$, gas sensors, lithium ion batteries and biomedical systems. However, the widespread technological use of titania is impaired by its wide band gap $(3.2 \mathrm{eV})$, which requires ultraviolet (UV) irradiation for photocatalytic activation. Because UV light accounts for only a small fraction (8\%) of the sun's energy compared to visible light (45\%), any shift in the optical response of titania from the UV towards full spectrum light will have a positive impact on the photocatalytic and photoelectrochemical utility of the material. Traditionally, doping of the titania has been the approach taken for its band-gap engineering ${ }^{64}$. When employing dopants to change the optical response of a material, it is desirable to maintain the integrity of the host material crystal structure, while changing its electronic structure. The crystal structure of the material is directly related to the ratio of cation and anion size in the crystal lattice.

Here, we propose an alternate way to enhance the electrical conductivity of $\mathrm{TiO}_{2}$ using mechanical straining. A recent theoretical work on boron nitride nanotubes (BNNTs) under flattening deformation has predicted the unique possibility of band gap tuning in a 2-5 eV range. This theoretical prediction on BNNT's has been experimentally verified by Bai et al. ${ }^{65}$ by a serious in-situ scanning tunneling microscopy (STM) experiments in a transmission electron microscope (TEM).

Titania crystallizes into three natural phases: brookite (orthorhombic), anatase (tetragonal) and rutile (tetragonal). Contrary to $\mathrm{TiO}_{2}$ films, where three distinct polymorphs of rutile, anatase and brookite are reported, the $\mathrm{TiO}_{2}$ nanotubes are reported to be either amorphous or anatase. ${ }^{66}$ The crystal structure of the $\mathrm{TiO}_{2}$ phase affects the photoelectrical current in solar cells and anatase based solar cells are expected to have the highest conversion efficiency. ${ }^{67}$ This effect has been attributed to the higher Fermi level in anatase in comparison to that of rutile by about $0.1 \mathrm{eV}$.

In view of this, we report here, the effect of mechanical deformation on an electrical response of an individual anatase $\mathrm{TiO}_{2}$ nanotubes. To the authors' 
knowledge, such experiments have never been conducted on these nanotubes before.

\subsubsection{Experimental Procedure}

The in-situ electrical measurements were conducted in a high resolution TEM using a STM-TEM holder from "Nanofactory Instruments". The holder consists of a STM equipped TEM sample holder, a controller and a PC with Nanofactory's data acquisition software. All the measurements were carried out on a single tilt STM-TEM holder in a JEM 4000FX TEM, operated at $200 \mathrm{keV}$. The setup of the STM-TEM holder is shown in Fig. 5.1.

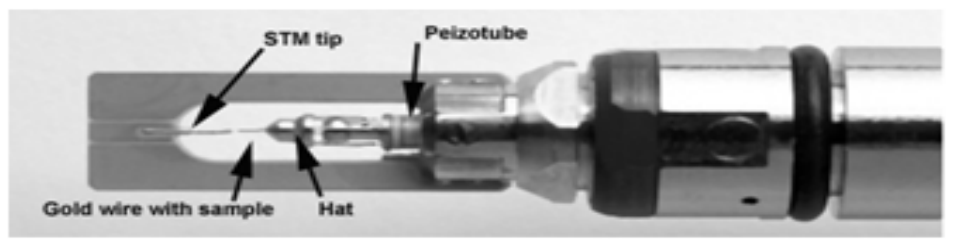

Figure 5.1. The image showing the STM-TEM holder used for present electrical measurements.

The electrochemically etched gold wire with $\mathrm{TiO}_{2}$ nanotubes was attached to the piezo-driven movable part of the holder facing the fixed and sharp tungsten STM tip as its counter electrode, and oriented perpendicular to the electron beam in the TEM. In such an arrangement, atomic scale imaging and I-V measurements can be carried out concurrently. The contact was made between the STM tip and the $\mathrm{TiO}_{2}$ nanotube by the precision movement of the gold wire (with the sample) attached to the piezo-driven manipulator. The electrical measurement was done by applying the bias voltages to the gold electrode with the nanotube sample at its tip, while the tungsten STM tip was grounded. In the present investigations, $\mathrm{TiO}_{2}$ nanotube samples were synthesized by anodization of $\mathrm{TiO}_{2}$ foils followed by calcinations of nanotubes at $450{ }^{\circ} \mathrm{C}$. Details of the synthesis process is reported elsewhere. ${ }^{68}$

\subsubsection{Results and Discussion}

The structural investigations of $\mathrm{TiO}_{2}$ nanotube samples were carried out 
during the in-situ TEM experiment. Fig. 5.2a shows an overall view of the anatase titania nanotube, depicting a number of tubular nanostructures with uniform size distribution. The tubes are hollow and open ended with an average inner and outer diameter of approximately $25 \mathrm{~nm}$ and $70 \mathrm{~nm}$ and the lengths range from several-tens to severalhundreds of nanometers. The corresponding diffraction pattern is shown in the inset (Fig. 5.2a), depicting the anatase titania ( $a=0.378 \mathrm{~nm}, c=0.9513 \mathrm{~nm}, \mathrm{I}_{1} / \mathrm{amd}$ ). The HRTEM image (Fig. 5.2b) taken from a single nanotube (inset of Fig. 5.2b) shows that the nanotubes are well crystalline. The lattice fringe spacing of the walls of the nanotubes is estimated to be $\sim 0.353 \mathrm{~nm}$, corresponding to the interplanar distance of the (101) plane in the anatase phase.

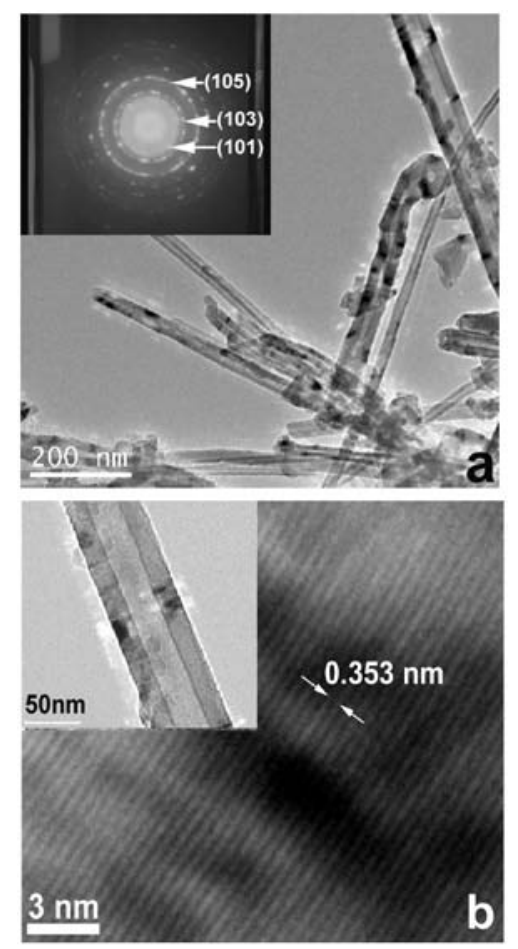

Figure 5.2. (a) An overall view of the anatase titania nanotube and the corresponding diffraction pattern (inset), and (b) the high resolution lattice image from a single anatase nanotube (inset).

To ensure good electrical contact between the tip and the nanotube for in-situ electrical measurement, an individual $\mathrm{TiO}_{2}$ nanotube was attached to the 
electromechanically etched gold tip by tungsten deposition using the focused ion beam (FIB) technique. The different steps of the sample preparation are shown in Fig. 5.3(a-c). In short, a nanotube was picked up using the FIB probe (Fig. 5.3a, Fig. 5.3b) and attached on the tungsten tip (Fig. 5.3c) by the tungsten deposition. The tungsten tip with $\mathrm{TiO}_{2}$ nanotube was then transferred to the STM-TEM specimen holder and approached to its opposite conducting STM tip by the peizo-manipulator. A schematic diagram of the experimental setup is shown in Fig. 5.3d. The gold wire with nanotube is attached to the moveable peizodriven stage while the fixed tungsten STM tip is its counter electrode.

The gold wire (with sample) positions can be easily moved in $\mathrm{X}, \mathrm{Y}$ and $\mathrm{Z}$ direction within the limited range to make a good contact of the nanotube with the STM tip.
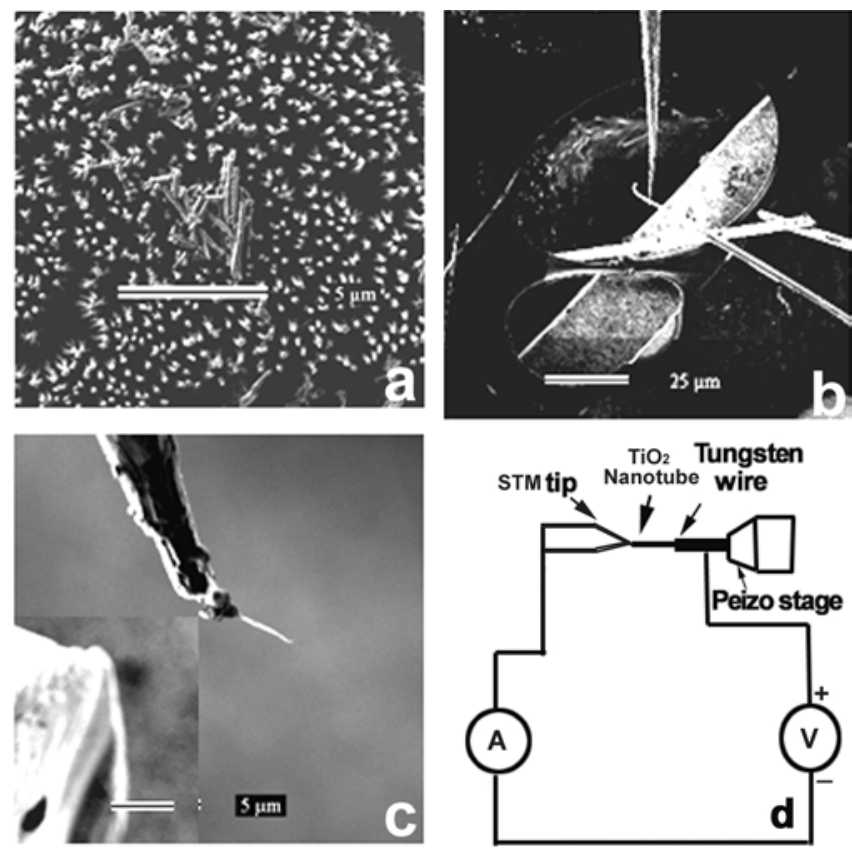

Figure 5.3. Images from the FIB system show (a) the as grown $\mathrm{TiO}_{2}$ nanotube sample; (b) FIB probe attached with a single nanotube, dispersed on the $\mathrm{Cu}$ mesh; (c) the FIB probe with a nanotube approaching the tip of the tungsten wire and; (d) schematic of the experimental setup for current-voltage measurement.

For electrical measurement, it is foremost to make a good physical contact of the nanotube with the two opposite electrodes. Under the present investigations, when the 
nanotube was brought in contact with the STM tip by the nanomanipulator, a current of several nA was allowed to pass through the electrode-nanotube circuit. Due to the passage of this current for a very short time (less than 2 seconds), the temperature of the contact point between the nanotube and the STM tip will increase and the nanotube is welded to the STM tip, which aids in making a good physical contact of the nanotube with the STM tip. Fig. 5.4a shows a $\mathrm{TiO}_{2}$ nanotube attached with the gold electrode and approaching the opposite electrode i.e STM tip by the nanomanipulator. Fig. 5.4b displays an image, when the $\mathrm{TiO}_{2}$ nanotube comes in contact with both the electrode and free standing in high vacuum $\left(10^{-6}\right.$ Torr) inside the TEM. Fig. 5.4(c-e) display the sequential images of a typical $\mathrm{TiO}_{2}$ nanotube undergoing a gradual increase in its bending curvature by incremental movement of the peizodriven gold tip toward the STM tip.

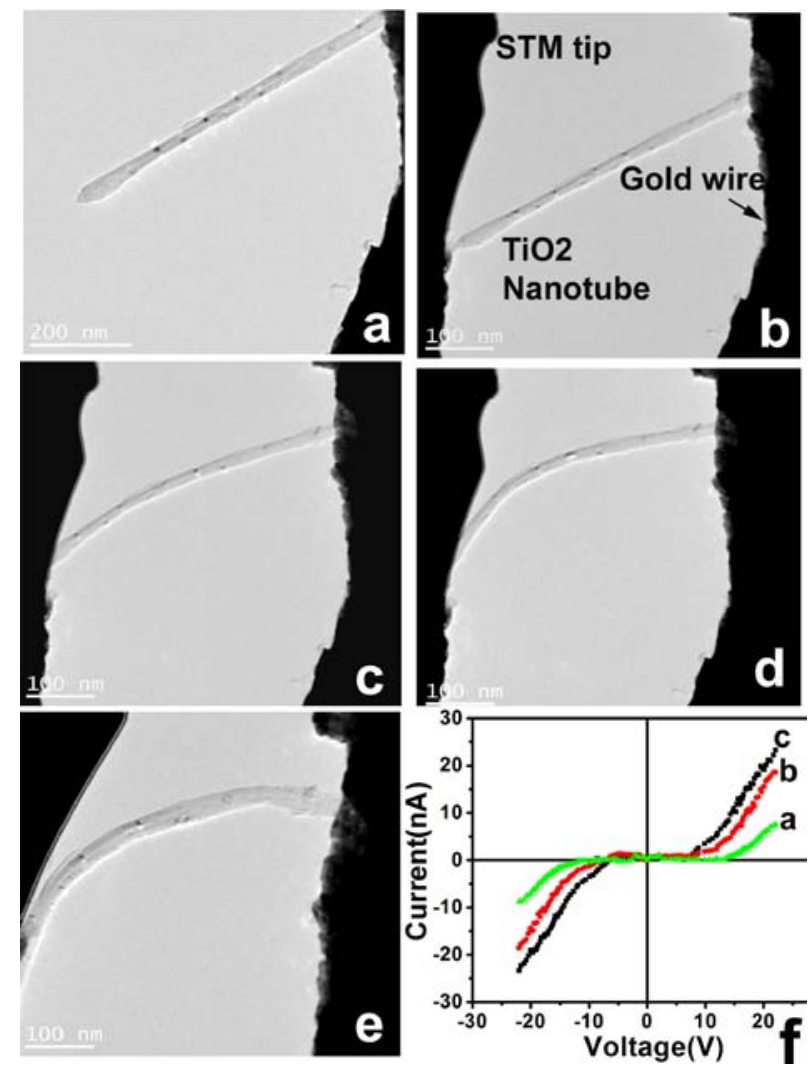

Figure 5.4. The bight field image of the $\mathrm{TiO}_{2}$ nanotube (a) approaching the STM tip; (b) in contact with the STM tip; (c) (d) \& (e) undergoing a gradual increase in its bending curvature; (f) a series of the representable I-V curves measured during the deformation of the $\mathrm{TiO}_{2}$ nanotubes. 
A series of measured $I-V$ curves at various stages of bending deformation are respectively shown in Fig. 5.4f. The $\mathrm{TiO}_{2}$ nanotube in contact with the STM tip (curve "a" in Fig. 5.4f) shows a semiconducting behavior where electrical currents up to $10 \mathrm{nA}$ can be detected under bias voltages up to $25 \mathrm{~V}$. This is due to the intrinsic semiconducting behavior of the $\mathrm{TiO}_{2}$ nanotube under the applied voltage. As we deform the nanotube by delicate driving of the tungsten tip with the nanotube against the STM tip, current up to $18 \mathrm{nA}$ can be observed (curve "b") for the deformed state corresponding to Fig. 5.4d. With the increase in deformation, the current is dramatically increased to 25 nA with start off voltage of $7.5 \mathrm{~V}$ bias as is evident from curve "d" (Fig. 5.4f). Fig. 5.4e displays the $\mathrm{TiO}_{2}$ nanotube in the highest deformed state under the present study, making a large bending curvature and corresponds to the state of the I-V curve " $\mathrm{d}$ ". In a large bias regime, the $I-V$ curve can be differentiated to obtain a resistance $R$ of the nanotube $(R \sim$ $\mathrm{d} V / \mathrm{d} I$,). We found that for this deformed state, the resistance of the nanotube was decreased to $0.34 \mathrm{G} \Omega$ from $\sim 0.86 \mathrm{G} \Omega$ in the state corresponding to Fig. $5.4 \mathrm{a}$.

The nonlinear and symmetrical I-V characteristics of these deformed states suggests a semiconducting behavior. Thus our measurement system can be regarded as a metal-semiconductor-metal (M-S-M) circuit. The related semiconducting parameters can be retrieved from the experimental $I-V$ data in the bias regime $>8 \mathrm{~V}$, by the following relation $^{69}$,

$$
\ln I=\ln S+V\left(\frac{q}{k_{B} T}-\frac{1}{E_{0}}\right)+\ln J_{s} .
$$

Here $\mathrm{S}$ is the contact area associated with a bias, $J_{\mathrm{S}}$ is slowly varying function of the applied bias. The $\ln I$ versus $V$ plot gives an approximately straight line with a slope of, $\left(q / K_{B} T\right)-1 / E_{0}$ and an intercept of $\ln S$. The representative $(\ln I)-V$ curves are depicted in Fig. 5.5a and 5.5b corresponding to curve $b$ and curve $c$ in Fig 5.4f, respectively. Fig. 5.5c shows the linear fits of curve $\mathrm{b}$ and $\mathrm{c}$ extrapolated to the $\ln \mathrm{I}$ axis, showing nearly identical values of intercept. 

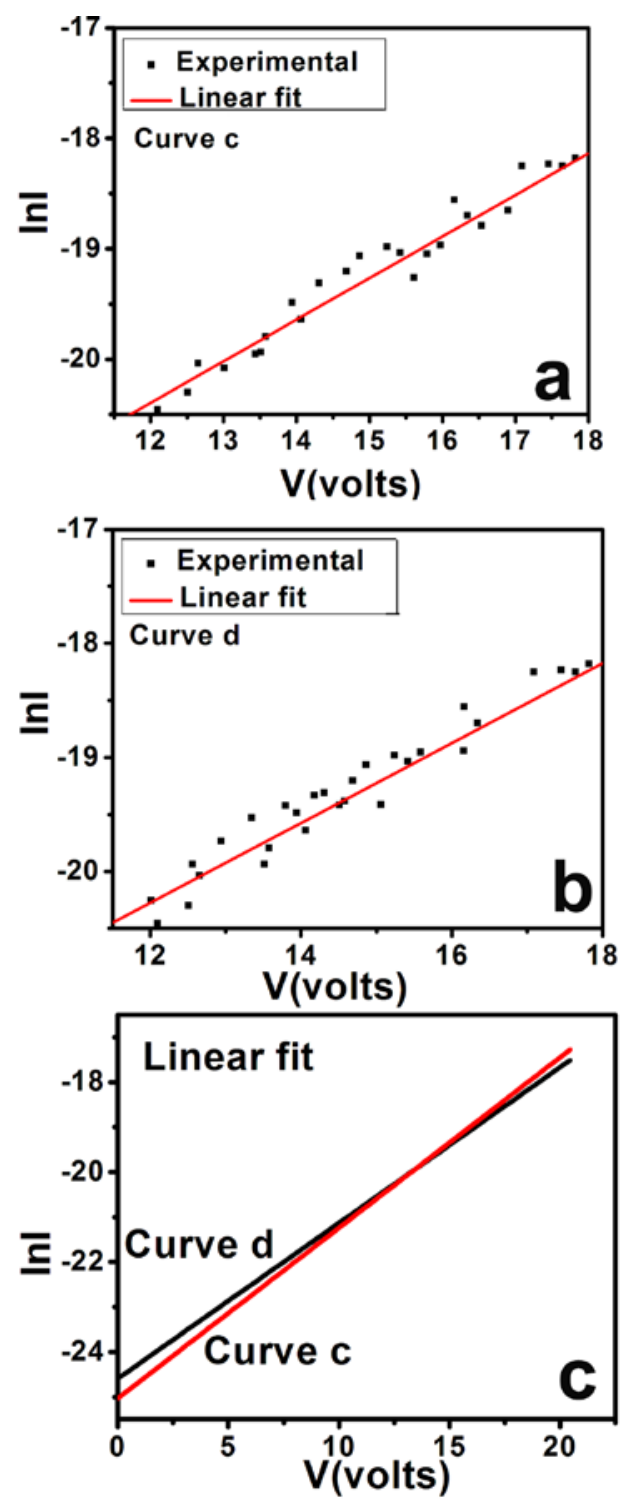

Figure 5.5. The $\ln I-V$ curves corresponding to (a) curve $b$ and (b) curve $c$ in Fig. 5.4f, respectively. (c) The linear fits of the curves in (a) and (b).

This means, $\ln S$ and the contact area $(S)$ is nearly identical for both of these deformed states. In the expression of $\ln I, E=E_{00} \operatorname{coth}\left(E_{00} / k B T\right)$

Where,

$$
E_{00}=(h q / 2)\left[n /\left(m^{*} \varepsilon\right)\right]^{1 / 2}
$$


Here, $q$ is the elemental charge, $k_{B}$ is the Boltzmann constant, $m^{*}$ is an effective electron mass of $\mathrm{TiO}_{2}$ nanotube and $\varepsilon$ is the dielectric constant. We estimated the specific sizes of the nanotube from the bright field TEM image, and thus the resistivity, $\rho$ was

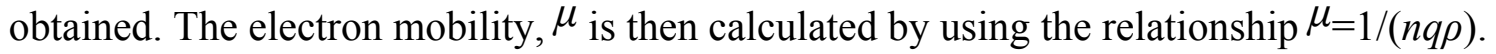

For $\mathrm{TiO}_{2}$ materials $\varepsilon=8.4 \varepsilon_{0}, J_{0}$ is the dielectric constant of a vacuum and $m *=0.8 m_{0}{ }^{28}$. Based on this procedure, the resistance, resistivity, carrier concentration and carrier mobility were extracted as summarized in Table 5.1. As shown, nanotube under the higher deformation has approximately 2.2 times higher charge carrier density but $\sim 1.7$ times lower charge mobility. The I-V measurements with bending deformation were repeated for several times to ensure the reproducibility of the data.

Table 5.1. Electrical parameters of $\mathrm{TiO}_{2}$ nanotube

\begin{tabular}{lccc}
\hline Parameters & Curve b & Curve c & Curve d \\
\hline Resistance $(\mathrm{G} \Omega):$ & 0.86 & 0.66 & 0.34 \\
Resistivity $(\Omega \mathrm{cm}):$ & 43.5 & 36.4 & 16.36 \\
$\mathrm{E}_{0}(\mathrm{meV}):$ & 23.98 & 24.9 & 27.0 \\
Carrier concentration $/(\mathrm{cm} 3):$ & $0.78 \times 10^{17}$ & $1.58 \times 10^{17}$ & $3.5 \times 10^{17}$ \\
Mobility $\left(\mathrm{cm}^{2} / \mathrm{Vs}\right)$ & 4.68 & 3.98 & 2.05 \\
\hline
\end{tabular}

Fig. 5.6a shows the $\mathrm{TiO}_{2}$ nanotube in the relaxed state after the unloading. The high resolution TEM image corresponding to the relaxed state is shown in Fig. 5.6b. It is evident from the high resolution image that the outer walls of the nanotube are partly broken, but most part of the nanotube is intact. In the present investigation, $\mathrm{TiO}_{2}$ nanotubes that were bent over the elastic limit were broken. Fig. 5.6c displays a part of the $\mathrm{TiO}_{2}$ nanotube bent over the elastic limit and shows the breaking and flattening of the walls. 

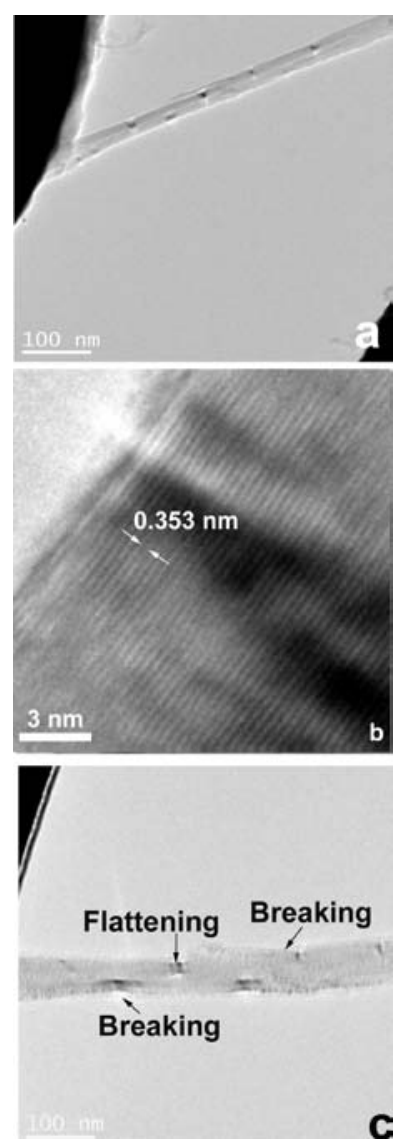

Figure 5.6. The bight field image of the $\mathrm{TiO}_{2}$ nanotube (a) in a relaxed state after the unloading (b) The high resolution TEM image corresponding to the relaxed state (c) undergoing the bending deformation process beyond the elastic limit.

Under the present study, it was interesting to note that the electrical conductivity of $\mathrm{TiO}_{2}$ nanotubes can be modulated by the mechanical deformation. Such phenomena can be related to the strain engineering of the electronic band gap structure in nanotubes ${ }^{70}$. It can be said that in the present study, when the anatase $\mathrm{TiO}_{2}$ nanotubes are brought into deformed state, in-shell defects are produced at the walls of the nanotube. The possible defects can be voids, vacancies, and antisite atoms, which modify the band structure $^{71}$. The carriers of current, i.e electrons in this case (as $\mathrm{TiO}_{2}$ is considered as $\mathrm{n}$ type semiconductor) are scattered strongly by the disordered structure, so that the mean free path of electrons may sometime be the order of the scale of the disorder and the current flows through the $\mathrm{TiO}_{2}$ nanotube. The pinning of the Fermi level is known to 
arise from the presence of defects ${ }^{72}$, such as dangling bonds and other misfits in the structure, which produce localized state in the gap. Hence, the $\mathrm{TiO}_{2}$ nanotube in a higher deformed state will produce more defects, so a large number of electrons are scattered by the formation of a large number of defects. The production of defects will also help in the pinning of the Fermi level, which will produce a localized state in the gap and hence help in the transport of the electrical current through the $\mathrm{TiO}_{2}$ nanotube.

Abther possibility for the increase of current by deformation can be due to the presence of dangling bonds at the surface of the $\mathrm{TiO}_{2}$ nanotube. These defects can have a dominant role in modulating its electrical conductivity. ${ }^{73}$ Surface defects can produce surface states within the band gap making the $\mathrm{TiO}_{2}$ nanotube behaves like a weakly conductive metal. This allows the flow of conduction electrons near the surface region of the $\mathrm{TiO}_{2}$ nanotube as also reported by Lin et al. ${ }^{74}$

It has been reported that, $\mathrm{TiO}_{2}$, being an ionic transition-metal oxide, exhibits strong electron-phonon coupling, resulting in low room temperature electron mobilities in the material. ${ }^{75}$ So, we believe that one of the other possible reason for the increase of electrical conductivity is due to the electron-phonon coupling. This electron-phonon coupling effects the formation of a polaron, a quasi-particle consisting of an electron and the accompanying lattice deformation. These polarons display self-trapping of the electron and localized wavefunctions, with charge transport typically occurring through tunnelling or thermally activated hopping.

\subsubsection{Conclusions}

In conclusions, we have successfully carried out in-situ electrical transport measurements on an individual anatase $\mathrm{TiO}_{2}$ nanotube inside a high resolution transmission electron microscope equipped with the STM probe. It was shown for the first time that the electrical transport properties of the $\mathrm{TiO}_{2}$ nanotube could be enhanced by inducing deformation into the nanotube using an in-situ STM-TEM stage. The semiconducting parameters were retrieved from the experimental I-V curves using the MS-M model. Here, it can be emphasized that, considering the deformation driven electrical property modulation of the $\mathrm{TiO}_{2}$ nanotube, as observed in the present 
investigations, the $\mathrm{TiO}_{2}$ nanotube holds a promising future and perspective candidate for constructing nanoscale electronic and optoelectronic devices and more importantly for its usage in solar cell applications. 


\title{
5.2. Deformation-Driven Electrical Transport in Amorphous $\mathrm{TiO}_{2}$ Nanotubes
}

\author{
A. Asthana, T. Shokuhfar ${ }^{3}$, Q. Gao, P. A. Heiden, C. Friedrich, R. S. Yassar
}

\begin{abstract}
A series of in-situ transmission electron microscopy combined with scanning tunneling microscopy measurements were carried out to investigate the effect of mechanical deformation on the electrical transport properties of an amorphous $\mathrm{TiO}_{2}$ nanotubes. Under no mechanical straining, it was found that $\mathrm{TiO}_{2}$ nanotubes behave as an electrical insulator. However, the nanotubes show semiconducting behavior under highly deformed state. On the basis of a metal-semiconductor-metal model, it was suggested that in-shell defects, surface defect-driven conduction modes and electron-phonon coupling effect are responsible for the appearance of semiconducting behaviors.
\end{abstract}

Key Words: $\mathrm{TiO}_{2}$ nanotubes, Amorphous, Electrical transport, In-situ TEM

\subsubsection{Experimental Procedure}

All the electrical measurements were carried out on a single tilt scanning tunneling microscopy (STM)-TEM holder in a JEM 4000FX TEM system that operated at $200 \mathrm{KeV}$. In the present investigations, $\mathrm{TiO}_{2}$ nanotube samples were synthesized by anodization of $\mathrm{TiO}_{2}$ foils. Details of the synthesis process are reported elsewhere. ${ }^{76}$

The structural investigations of $\mathrm{TiO}_{2}$ nanotube samples were carried out during the in-situ TEM experiment. Fig. 5.7a is a low magnification bright field image of the assynthesized amorphous $\mathrm{TiO}_{2}$ nanotube. The general morphology shows that the amorphous $\mathrm{TiO}_{2}$ nanotubes are hollow and closed ended. The nanotubes have average outer and inner diameters of $100 \mathrm{~nm}$ and $50 \mathrm{~nm}$, and the wall thickness of the tubes is not uniform. The corresponding diffraction pattern (inset in Fig. 5.7a) and the high-resolution

${ }^{3}$ I have contributed to this publication by brainstorming the idea and synthesizing the main material of characterization ( $\mathrm{TiO}_{2}$ nanotubes). 
transmission electron microscopy (HRTEM) image (Fig. 5.7b) clearly show that the tubular structures are amorphous.

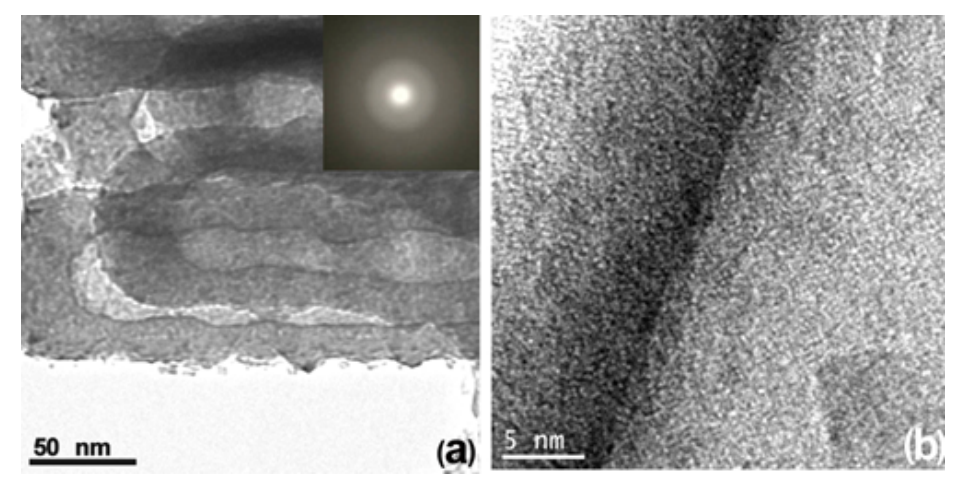

Figure 5.7. (a) TEM bright field (BF) image of the amorphous $\mathrm{TiO}_{2}$ nanotubes and its corresponding electron diffraction pattern (inset) and (b) the high-resolution lattice image.

For in-situ electrical measurement, individual $\mathrm{TiO}_{2}$ nanotubes were attached to the electromechanically etched tungsten tip by tungsten deposition using the focused ion beam (FIB) technique to ensure good electrical contact between the tip and the nanotubes. The different steps of the sample preparation are shown in Figs. 5.8(a-c). In short, a nanotube was picked up using the FIB probe (Fig. 5.8a and b) and attached on the tungsten tip (Fig. 5.8c) by the tungsten deposition. The tungsten tip with $\mathrm{TiO}_{2}$ nanotube was then transferred to the STM-TEM specimen holder and approached to its opposite conducting STM tip by the peizo-manipulator. A schematic diagram of the experimental setup is shown in Fig. 5.8d. 

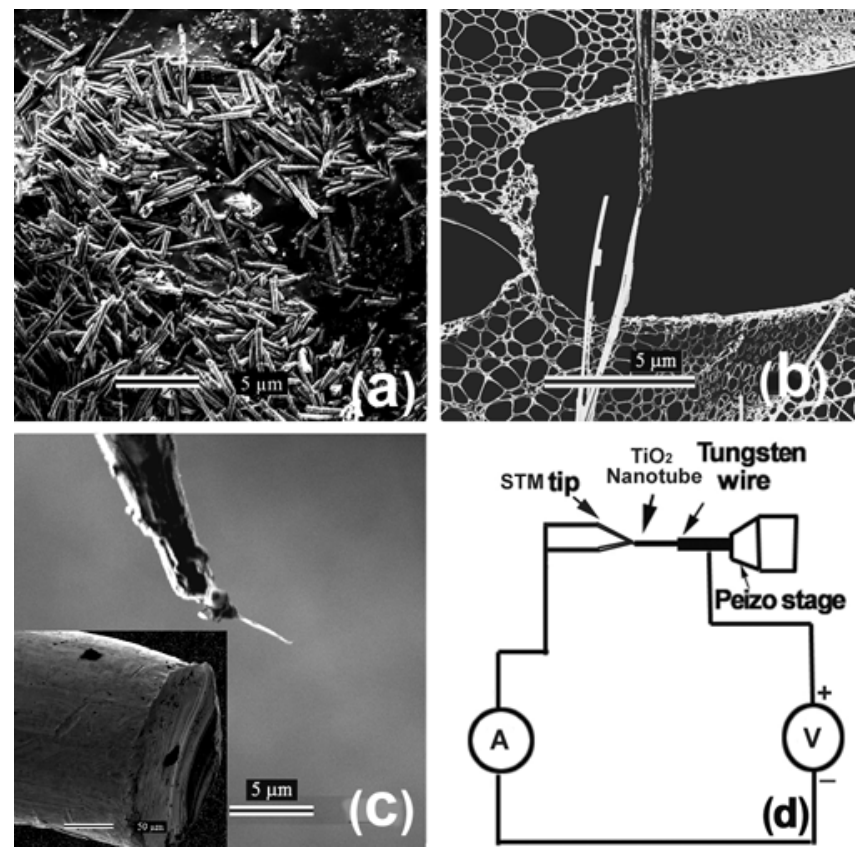

Figure 5.8. Images from the FIB system showing (a) the as grown $\mathrm{TiO}_{2}$ nanotube sample;

(b) FIB probe attached with a single nanotube, dispersed on the $\mathrm{Cu}$ mesh (c) the FIB probe with a nanotube approaching the tip of the tungsten wire and (d) Schematic of the experimental setup for current - voltage measurement.

\subsubsection{Results and Discussion}

A series of measured $I-V$ curves at various stages of bending deformation are respectively shown in Fig. 5.9. For the $\mathrm{TiO}_{2}$ nanotube that is just in contact with the STM tip and the nanotube that is in a slightly deformed state, the current is practically negligible, with a current of only a few $n A$ able to be detected (curves " $a$ " and " $b$ " respectively in Fig. 5.9f). This result confirms the insulating behavior of undeformed or just slightly deformed nanotubes, and can be attributed to less contact area, high contact resistance and the intrinsic amorphous nature of the material.

The amorphous materials possess high resistance due to their internal molecular disorder, as compared to the more orderly crystal structure of crystalline materials. Fig. 
5.9c shows the $\mathrm{TiO}_{2}$ nanotube in a slightly deformed state during the compression process. As we deform the nanotube by delicate driving of the tungsten tip with the nanotube against the STM tip, currents up to 8 and $14 n A$ can be observed at the same bias voltage range (curves " $c$ " and " $d$ "). In a large bias regime, the $I-V$ curve can be differentiated to obtain a resistance $R$ of the nanotube $(R \sim d V / d I)$. The nanotube's resistance, $R$, for this state was calculated to be $\sim 2.0 \mathrm{G} \Omega$ (Curve " $c$ "). With the increase in deformation, the current is dramatically increased at the start off bias voltage of $7.5 \mathrm{~V}$ as is evident from Curve " $d$ " (Fig. 5.9f). Fig. 5.9e displays the $\mathrm{TiO}_{2}$ nanotube in the highest deformed state under the present study, and the corresponding $I-V$ curve is marked as " $d$ ". We found that for this highly deformed state, the resistance of the nanotube was decreased to $0.98 \mathrm{G} \Omega$. The comparison of these values indicates that the resistance of $\mathrm{TiO}_{2}$ nanotubes decreases as mechanical straining increases.
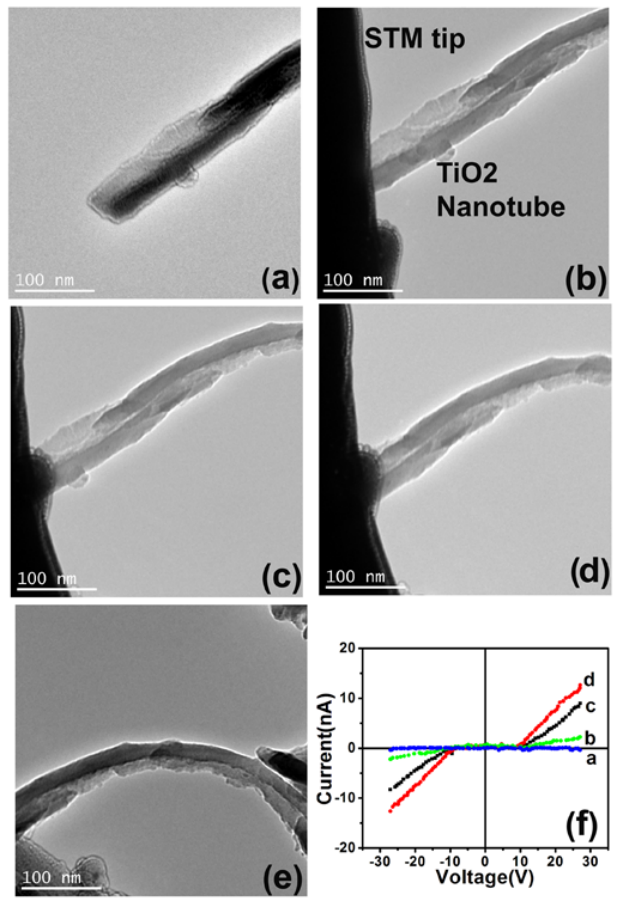

Figure 5.9. The bight field image of the $\mathrm{TiO}_{2}$ nanotube (a) approaching the STM tip; $(b)$ in contact with the STM tip; (c) (d) \& (e) undergoing a gradual increase in its bending curvature; (f) a series of the representable I-V curves measured during the deformation of the $\mathrm{TiO}_{2}$ nanotubes. 
The nonlinear and symmetrical $I-V$ characteristics of these deformed states suggests for a semiconducting behavior. Thus our measurement system can be regarded as a metal-semiconductor-metal $(M-S-M)$ circuit. The related semiconducting parameters can be retrieved from the experimental $I-V$ data in the bias regime $>5 \mathrm{~V}$, by the following relation,

$$
\ln I=\ln S+V\left(\frac{q}{k_{B} T}-\frac{1}{E_{0}}\right)+\ln J_{s}
$$

Here $S$ is the contact area associated with a bias, $J_{s}$ is a slowly varying function of the applied bias. The $\ln I$ versus $V$ plot gives an approximately straight line with a slope of,

$$
\left(\frac{q}{k_{B} T}\right)-\frac{1}{E_{0}}
$$

and an intercept of $\ln S$. The representative $(\ln I)-V$ curves are depicted in Fig. 5.10a and 5.10b corresponding to Curve $c$ and Curve $d$ in Fig 5.9f, respectively. Fig. 5.10c shows the linear fits of curves $c$ and $d$ extrapolated to the $\ln I$ axis, and shows that they have nearly identical intercept values. This means that the $\ln S$ and the contact area $(S)$ are nearly identical for both of these stressed states. In the expression of $\ln I$,

$$
E_{0}=E_{00} \operatorname{coth}\left(\frac{E_{00}}{k_{B} T}\right)
$$

where,

$$
E_{00}=\left(\frac{\hbar q}{2}\right)\left(\frac{n}{m^{*} \varepsilon}\right)^{1 / 2} \quad \mu=\frac{1}{(n q \rho)} .
$$

Here, $q$ is the elemental charge, $k_{B}$ is the Boltzmann constant, $m *$ is an effective electron mass of $\mathrm{TiO}_{2}$ nanotube and $\varepsilon$ is the dielectric constant. We estimated the specific sizes of the nanotube from the bright field TEM images and thus the resistivity, $\rho$, was 
obtained. The electron mobility, $\mu$, was then calculated by using the relationship,
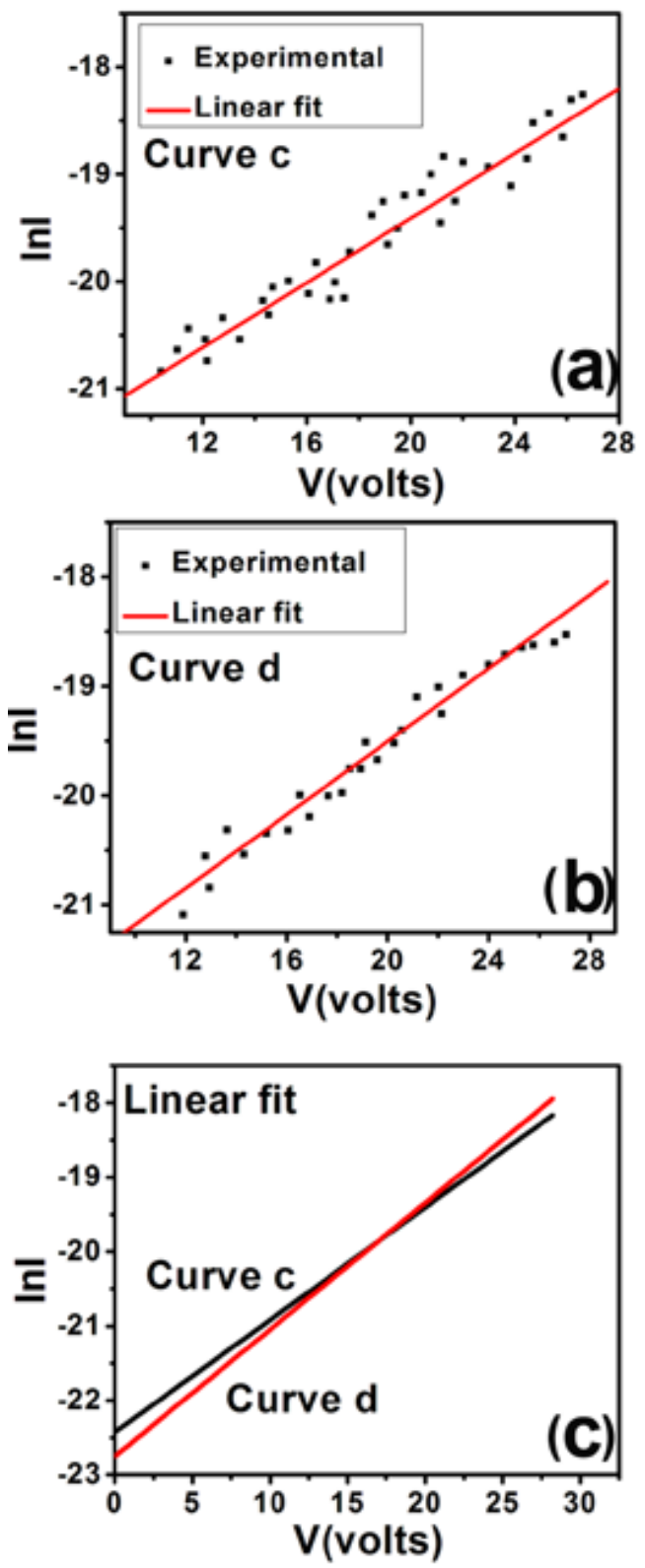

Figure 5.10. The $\ln I-V$ curves corresponding to (a) Curve $c$ and Curve $d$ in Fig. 5.9f, respectively. (c) The linear fits of the curves in (a) and (b).

For $\mathrm{TiO}_{2}$ materials, $\varepsilon=5.87 \varepsilon_{0}$, where $J_{0}$ is the dielectric constant of a vacuum and $\mathrm{m}^{*}=0.71 \mathrm{~m}_{0}$. Using this procedure, the resistance, resistivity, carrier 
concentration and carrier mobility were extracted, and are summarized in Table 5.2. Although the resistance of the amorphous nanotube is decreased upon deformation, it is still in the range of $\mathrm{G} \Omega$. An amorphous material has high resistance due to the disordered atomic-scale structure. As shown, a nanotube under a higher applied stress possesses $\sim 1.7$ times higher charge carrier density but $\sim 1.4$ times lower charge mobility. The I-V measurement with bending deformation were repeated for several times to ensure the reproducibility of the data.

Table 5.2. Electrical parameters of $\mathrm{TiO}_{2}$ nanotube

\begin{tabular}{lcc}
\hline Parameters & Curve $c$ & Curve $d$ \\
\hline Resistance $(\mathrm{G} \Omega):$ & 2.0 & 0.98 \\
$\mathrm{E}_{0}(\mathrm{meV}):$ & 22.98 & 23.0 \\
Carrier concentration $/(\mathrm{cm} 3):$ & $0.75 \times 10^{17}$ & $1.25 \times 10^{17}$ \\
Mobility $\left(\mathrm{cm}^{2} / \mathrm{Vs}\right):$ & 5.54 & 4.05
\end{tabular}

In the present study, the electrical conductivity of the amorphous $\mathrm{TiO}_{2}$ nanotube is tuned from that of an insulator to a semiconductor under the deformed state. This effect can be related to the modification of the band structure of the nanotube under strain. An amorphous $\mathrm{TiO}_{2}$ nanotube can be considered as an amorphous semiconductor. One possibility is that when the amorphous $\mathrm{TiO}_{2}$ nanotubes are subjected to mechanical straining, structural defects like voids, vacancies, and antisite atoms are produced inside the nanotube, which can alter the band gap properties. It is changes in the short-range order (on the scale of a localized electron) that have a profound effect on the properties of amorphous semiconductors. The carriers of current, i.e. electrons in this case $\left(\mathrm{as} \mathrm{TiO}_{2}\right.$ is considered as $n$ type semiconductor), are scattered strongly by the disordered structure, so that the mean free path of electrons may sometimes be on the order of the scale of the disorder and the current flows through the amorphous $\mathrm{TiO}_{2}$ nanotube. Also, amorphous materials appear to behave almost like intrinsic semiconductors with the Fermi 
level lying near the center of the gap. However the pinning of the Fermi level is known to arise from the presence of defects, such as dangling bonds and other flaws in the structure, which produce localized states in the gap. Hence the amorphous $\mathrm{TiO}_{2}$ nanotube in a higher deformed state will produce more defects, so a large number of electrons are scattered by the formation of a large number of defects. The production of defects will also help in the pinning of Fermi level and produce a localized state in the gap.

$\mathrm{TiO}_{2}$, being an ionic transition-metal oxide, exhibits strong electron-phonon coupling, resulting in low room temperature electron mobilities in the material. We, therefore, believe that an alternative possibility to increase electrical conductivity is the electron-phonon coupling effect. This electron-phonon coupling effects the formation of a polaron, a quasi-particle consisting of an electron and the accompanying lattice deformation. These polarons display self-trapping of the electron and localized wave functions, with charge transport typically occurring through tunneling or thermally activated hopping.

\subsubsection{Conclusions}

In conclusions, we have successfully carried out in-situ electrical transport measurements on an individual amorphous $\mathrm{TiO}_{2}$ nanotube inside a high-resolution transmission electron microscope equipped with the STM probe. It was shown for the first time that the electrical transport properties of the amorphous $\mathrm{TiO}_{2}$ nanotube could be tuned from the insulating to semiconducting by inducing deformation. The semiconducting parameters were retrieved from the experimental $I-V$ curves using the $M$ $S-M$ model. The considerable enhancement in electrical transport of amorphous $\mathrm{TiO}_{2}$ nanotubes offers new opportunities to build flexible or stretchable solar cells based on amorphous titania, where the conversion efficiency is comparable to anatase-based solar cells. If this can be integrated successfully, the annealing process of amorphous nanotubes can be eliminated from the solar cell manufacturing process, which can result in lower consumption of energy resources and saving costs. 


\section{Acknowledgement}

I would like to appreciate the funding support through the NSF-DMR grant no. 0820884 and NSF-CMMI grant no. 09268 


\title{
Chapter 6
}

\author{
6.1. Structural Instabilities in $\mathrm{TiO}_{2}$ Nanotubes \\ T Shokuhfar ${ }^{4}, Q$ Gao, A Ashtana, K Walzack, P Heiden, C Friedrich \\ Journal of Applied Physics. 108, 104310 (2010)
}

\begin{abstract}
We report the structural instability of $\mathrm{TiO}_{2}$ nanotubes subjected to treatment with ammonium hydroxide $\left(\mathrm{NH}_{4} \mathrm{OH}\right)$ solution prior to calcination at elevated temperatures. The nanotubes were disintegrated into nanoparticles and the tubular morphology was vanished after two hours of calcination at $500^{\circ} \mathrm{C}$. High-resolution transmission electron microscopy (TEM), Raman spectroscopy, x-ray diffraction (XRD), and atomic force microscopy (AFM) were used to understand the nature of structural collapse in the $\mathrm{NH}_{4} \mathrm{OH}$ treated $\mathrm{TiO}_{2}$ nanotubes. It was concluded that the volumetric changes during amorphous to anatase phase transformation and surface cracking was the key role during the collapse of $\mathrm{NH}_{4} \mathrm{OH}$-treated $\mathrm{TiO}_{2}$ nanotubes.
\end{abstract}

\subsubsection{Introduction}

Titanium dioxide $\left(\mathrm{TiO}_{2}\right)$ is a semiconductor with excellent photocatalytical properties, and the recent reports on fabrication of $\mathrm{TiO}_{2}$ nanotube layers (see e.g. Ref. ${ }^{77}$ ) have opened new perspectives for solar cell applications. The method to prepare such tubes, based on anodization of Ti foils in diluted HF-containing electrolytes, has been reported in recent years. The resulting $\mathrm{TiO}_{2}$ structures are composed of arrays of nanotubes with diameter of about $100 \mathrm{~nm}$.

The drawback is the high band gap energy $(\sim 3.2 \mathrm{eV})$ of $\mathrm{TiO}_{2}$, which means that it can be excited only by UV light $(\lambda<380 \mathrm{~nm}) .{ }^{78}$ Over the last decades, essentially two approaches were intensively investigated in order to make the material more responsive to the natural solar spectrum. One approach is to sensitize $\mathrm{TiO}_{2}$ with a suitable dye to

${ }^{4}$ As the first author of this publication I performed part of experimental studies and wrote the results, discussion, and conclusion. 
construct an efficient solar cell. The other approach to obtain a good photocatalytic performance is doping with impurities, e.g. transition metals or other elements, including $\mathrm{C}, \mathrm{N}, \mathrm{F}, \mathrm{P}$ and $\mathrm{S}$. It was found that substitutional N-doping is the most promising path towards photocatalytical applications. It has been suggested that Nitrogen ions substitute oxygen atoms in the $\mathrm{TiO}_{2}$ lattice and thus the corresponding $\mathrm{N}(2 \mathrm{p})$ states are located above the valence band edge. Mixing of $N(2 p)$ states with $O(2 p)$ states results in a reduction of the band gap of the N-doped $\mathrm{TiO}_{2}$ and as a result, higher photoelectrochemical efficiencies can be achieved under VIS light irradiation. ${ }^{79}$ The common approach to $\mathrm{N}$-dope $\mathrm{TiO}_{2}$ is its sputtering in a gas mixture of $\mathrm{N}_{2}$ with $\mathrm{Ar}^{80}$, annealing in pure ammonia gas ${ }^{81}, \mathrm{DC}$ magnetron sputtering of $\mathrm{TiO}_{2}$ electrodes in $\mathrm{Ar} / \mathrm{O}_{2} / \mathrm{N}_{2}$ mixture, or, alternatively, by immersing the as-anodized $\mathrm{TiO}_{2}$ nanotubes in nitrogen enriched media and then calcinations at high temperatures.

Before utilization of the titania nanotubes for different applications, factors like the crystalline nature of the structure and stability of the desired crystalline phases must be examined. Here, we report the structural instability of $\mathrm{TiO}_{2}$ nanotubes exposed to concentrated aqueous ammonia solution. High-resolution transmission electron microscopy (TEM), Raman spectroscopy, x-ray diffraction (XRD), and atomic force microscopy (AFM) were used to understand the nature of structural collapse in the $\mathrm{NH}_{4} \mathrm{OH}$ treated $\mathrm{TiO}_{2}$ nanotubes.

\subsubsection{Experimental Procedure}

\subsubsection{Synthesis}

Titanium foil (Alfa Aesar, $0.25 \mathrm{~mm}$ thick, 99.95\%) was used as the substrate for growth of the oxide nanotube arrays. All the reagents were used as provided, and the solutions were all prepared with deionized water obtained using a Milli-Q Reagent Water System (Millipore, Bedford, MA, USA). Prior to the anodization, the titanium foils were chemically polished in $1 \% \mathrm{HF}$ aqueous solution for $2 \mathrm{~min}$, rinsed with de-ionized water, and dried in nitrogen. The pretreated titanium foil was anodized by using platinum foil as cathode in an electrolyte containing dimethyl sulfoxide (DMSO) and 
hydrofluoric acid (HF, 48\% aqueous solution). The anodization voltage, HF concentration in DMSO, and duration of anodization were in the range of $60 \mathrm{~V}, 2 \mathrm{wt} \%$, and $40 \mathrm{hrs}$, respectively. Platinum foil was used as the counter electrode. After being anodized, specimens were rinsed with distilled water immediately and dried. The asanodized foils were then immersed in concentrated aqueous ammonium hydroxide $\left(\mathrm{NH}_{4} \mathrm{OH}\right)$ solution for $30 \mathrm{~min}$, and then calcinated at $500^{\circ} \mathrm{C}$ in atmosphere for $15-120$ min. The non-treated titania nanotubes were also annealed at the same condition for comparison purposes.

\subsubsection{Characterization}

The surface morphologies and length of the doped $\mathrm{TiO}_{2}$ nanotube array were observed by a field emission scanning electron microscopy (FE-SEM), and the crystallinity of the samples was investigated by $\mathrm{x}$-ray diffraction (XRD) technique using a Scintag XDS2000 Powder diffractometer. Raman spectra were collected at room temperature with a Raman spectrometer (Jobin-Yvon HR800 Raman Spectrometer) operating with $\mathrm{NeCd}$ laser of $442 \mathrm{~nm}$ with the resolution system of $\sim 0.1 \mathrm{~cm}^{-1}$. The crystallinity studies and elemental analysis of the titania nanotubes separated from the titanium support were performed using a JEOL-4000 FX TEM operated at $200 \mathrm{KeV}$.

\subsubsection{Results}

Figures 6.1a and $\mathrm{b}$ show the top-view SEM morphologies of the $\mathrm{TiO}_{2}$ nanotube arrays prepared by anodizing of titanium. The $\mathrm{TiO}_{2}$ nanotube arrays are tubes with diameter of 60-90 nm and a wall thickness of about $20 \mathrm{~nm}$. Most of the pore mouths of nanotube are open on the top of the layer. It can be seen that the nanotube arrays are uniform over the substrate. 

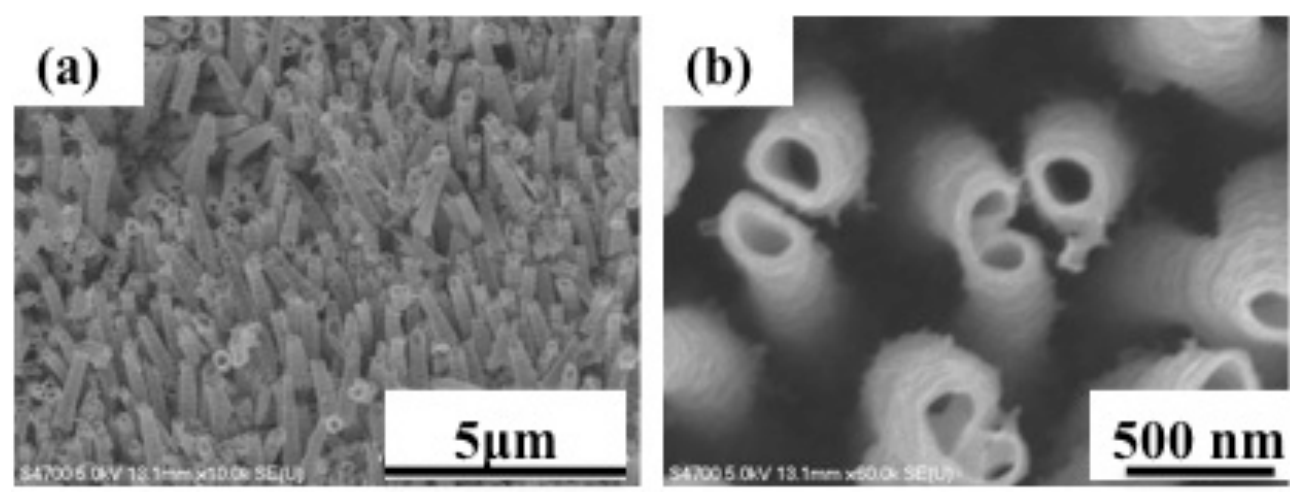

Figure 6.1. The SEM images of (a) as-synthesized $\mathrm{TiO}_{2}$ nanotubes arrays, and (b) close up of nanotubes shown in (a).

To reveal the morphological structure of as-synthesized $\mathrm{TiO}_{2}$ nanotubes, the samples were investigated by TEM. Figure 6.2 shows a bright field and corresponding diffraction pattern for the nanotubes. The tubular structure with variation in wall thickness throughout the length of the tube (marked by arrows) is evident from the figure. As one can see, the diffraction pattern (Figure 6.2b) shows diffuse peaks resembling the amorphous structures for the samples. A close examination of samples using high resolution imaging (Figure 6.2c) did not reveal any sign of distinct atomic order confirming the amorphous morphology of the as-synthesized tubes.

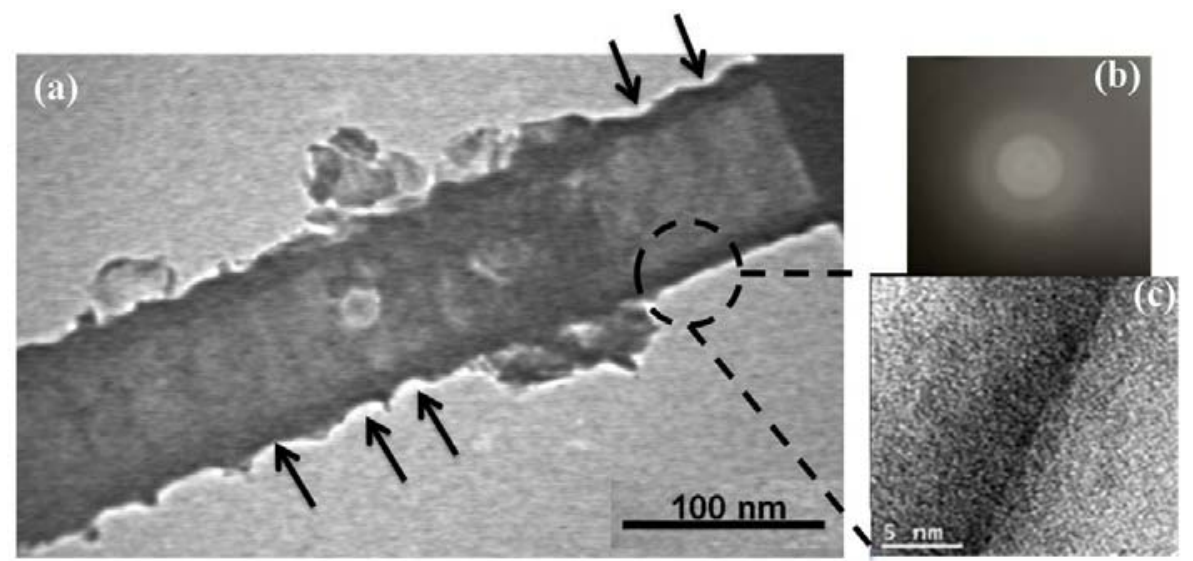

Figure 6.2. The bright field (a), diffraction pattern (b), and high-resolution TEM (c) images of as-synthesized $\mathrm{TiO}_{2}$ nanotubes.

In the next step, the samples were immersed in ammonium hydroxide solution 
for $30 \mathrm{~min}$. After $30 \mathrm{~min}$ the nanotubes were let to dry in air prior to SEM imaging. Figures 6.3a, and $\mathrm{b}$ show that the $\mathrm{NH}_{4} \mathrm{OH}$-treated nanotubes maintain their tubular morphology. The SEM analysis of end-on nanotubes did not reveal any changes in the tubes' diameter or length in comparison to the tubes shown in Figure 6.1.
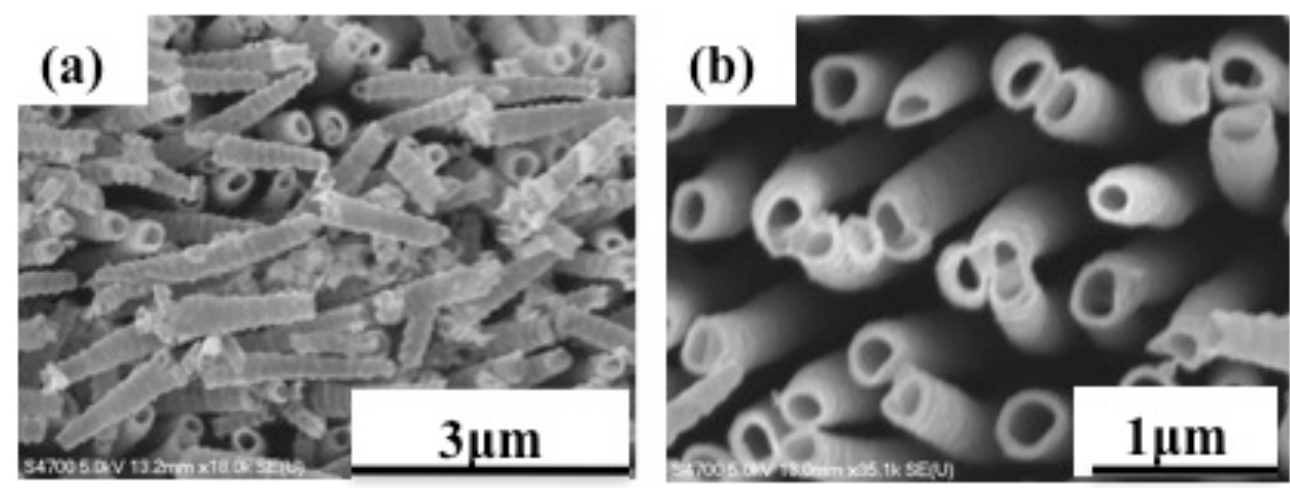

Figure 6.3. $(\mathrm{a}, \mathrm{b}) \mathrm{The} \mathrm{SEM}$ images of $\mathrm{TiO}_{2}$ nanotubes treated by $\mathrm{NH}_{4} \mathrm{OH}$ for $30 \mathrm{~min}$.

Both the as-synthesized and $\mathrm{NH}_{4} \mathrm{OH}$-treated $\mathrm{TiO}_{2}$ nanotubes were heated to $500^{\circ} \mathrm{C}$, which is the temperature that anatase should become thermodynamically stable. ${ }^{82}$ Figure 6.4 shows SEM, bight field TEM images, and corresponding diffraction pattern of the as-synthesized nanotubes after calcinations at 500C. As Figure 6.4a shows the annealing of as-synthesized nanotubes did not alter apparent morphology of the tubes (compare Figure 6.4a and 6.1a). However, the TEM diffraction pattern (Figure 4c) and high resolution imaging of the tube walls (Figure 6.4d) confirm the transformation of amorphous structure to anatase phase by calcination at $500^{\circ} \mathrm{C}$. 

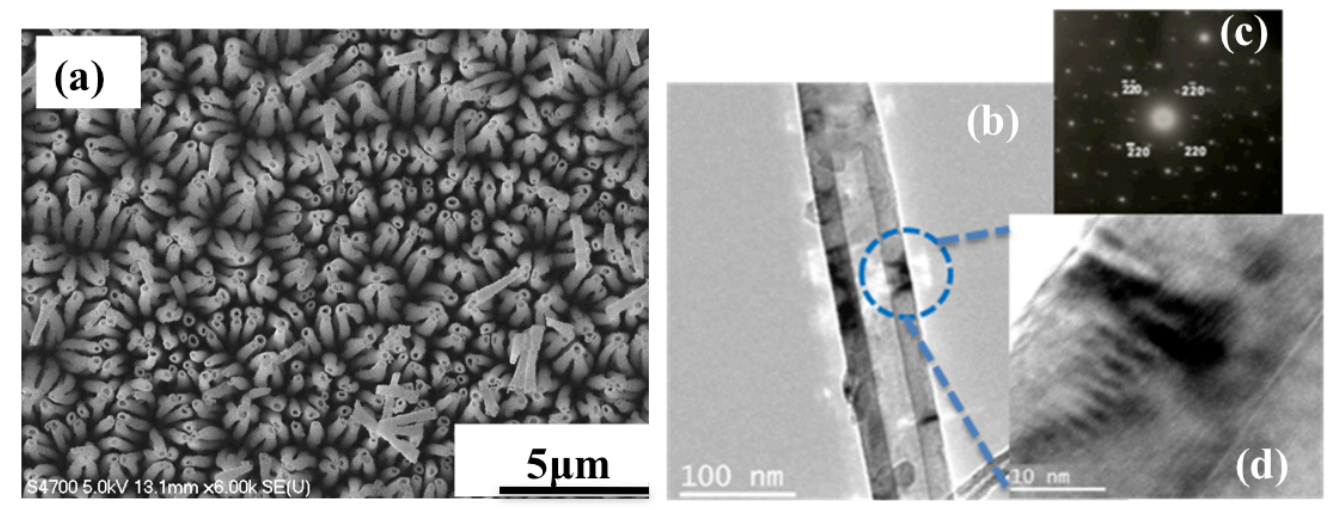

Figure 6.4. The SEM images show (a) as-synthesized $\mathrm{TiO}_{2}$ nanotubes after annealing in $500^{\circ} \mathrm{C}$. $(\mathrm{b}, \mathrm{c})$ The bright field TEM image and diffraction pattern of nanotubes shown in (a) indicate the transformation of amorphous morphology to crystalline order in the nanotubes. (d) The high resolution TEM image confirms the crystalline order of anatase phase and the presence of structural defects (appear as dark contrasts).

Interestingly, the structural studies of $\mathrm{NH}_{4} \mathrm{OH}$-treated $\mathrm{TiO}_{2}$ nanotubes annealed at $500^{\circ} \mathrm{C}$ revealed an unexpected morphological transformation. Figure 6.5 shows the SEM images of the $\mathrm{NH}_{4} \mathrm{OH}$-treated nanotubes. While the non-treated nanotubes maintain the same structural morphology (compare Figure 6.3a and 6.1a), the $\mathrm{NH}_{4} \mathrm{OH}$-treated sample showed no sign of nanotube architects. Surprisingly, the nanotubes were being replaced with nanoparticles of 20-30 $\mathrm{nm}$ size.

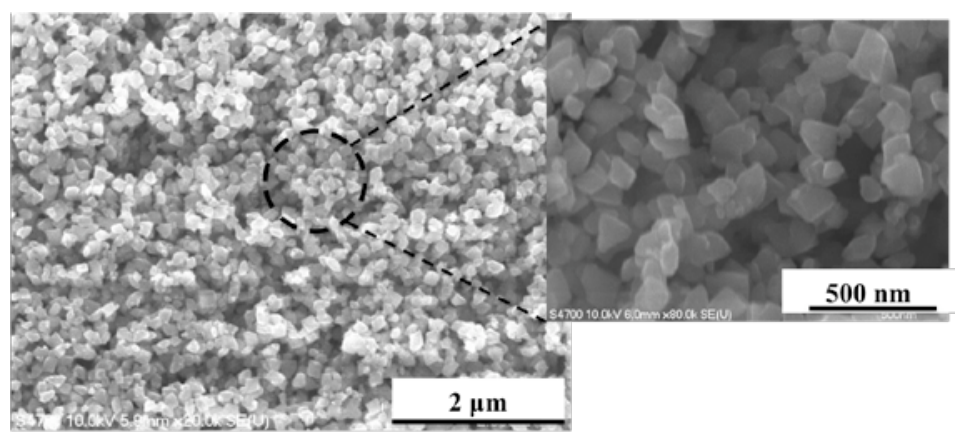

Figure 6.5. The SEM images show the morphology of the $\mathrm{NH}_{3}$-treated $\mathrm{TiO}_{2}$ nanotubes after calcination at $500^{\circ} \mathrm{C}$ for two hours. The treated nanotubes collapsed into nanoparticles upon heat treatment at anatase phase transformation temperature. 
To study the evolution of such structural transformation, a serious of calcination treatments at various time steps were carried out. Figure 6.6 shows the SEM images of $\mathrm{NH}_{4} \mathrm{OH}$-treated $\mathrm{TiO}_{2}$ nanotubes after 0,10 , and 30 minutes of calcination at $500^{\circ} \mathrm{C}$. As one can note, the morphology of $\mathrm{NH}_{4} \mathrm{OH}$ nanotubes is relatively unchanged. However after $10 \mathrm{~min}$ the nanotubes start to disintegrate into small particles and this behavior is more severe after $30 \mathrm{~min}$ where the nanotubes are barely maintain their tubular morphology.
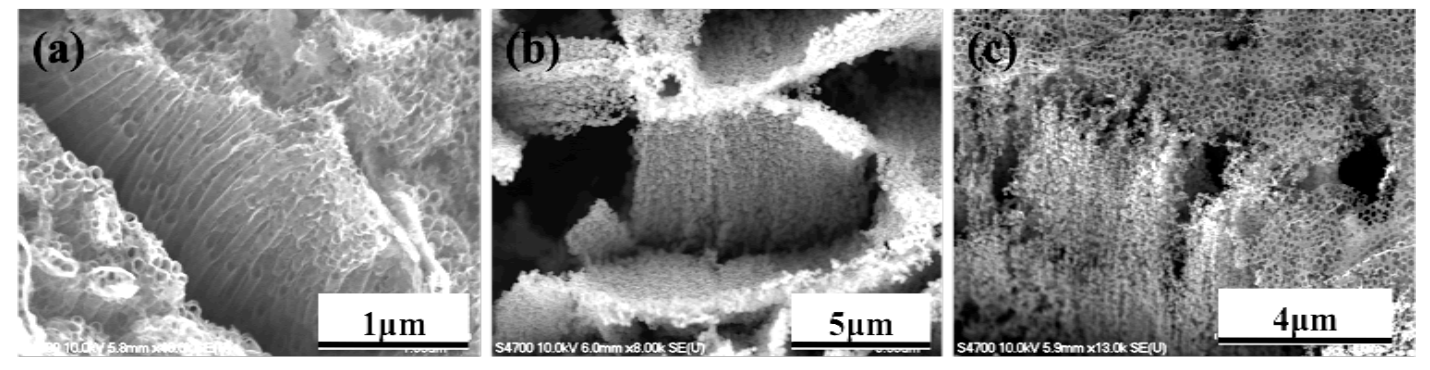

Figure 6.6. The $\mathrm{SEM}$ images of $\mathrm{NH}_{4} \mathrm{OH}$-treated $\mathrm{TiO}_{2}$ nanotubes after 0 (a), 10 (b), and 30 (c) minutes of calcination at $500^{\circ} \mathrm{C}$. The treated nanotubes start to disintegrate into small particles.

This unexpected structural change in the $\mathrm{NH}_{4} \mathrm{OH}$-treated $\mathrm{TiO}_{2}$ nanotubes motivated us to further study the role of ammonium hydroxided titanium dioxide nanotubes by XRD, Raman, and TEM. Figure 6.7 shows the XRD patterns for the untreated $\mathrm{TiO}_{2}$ and $\mathrm{NH}_{3}$-treated $\mathrm{TiO}_{2}$ nanotubes (designated as $(\mathrm{N})$ ) after annealing at 300 and $500^{\circ} \mathrm{C}$ temperatures. For samples annealed at $300^{\circ}$ and $500^{\circ} \mathrm{C}$ for $2 \mathrm{~h}$, the highest intensity diffraction peaks appeared at $25.43^{\circ}$ and $48.10^{\circ}$, in well accordance with the (101) and (200) peaks of anatase titania. This indicates the formation of crystalline anatase phase, whereas no evidence of the existence of rutile phase is observed. In $500^{\circ} \mathrm{C}$ sample, two weak peaks at $41.5^{\circ}$ and $53.5^{\circ}$ (marked by * symbol) appeared corresponding to Ti foil (the substrate). The $\mathrm{NH}_{4} \mathrm{OH}$ treated titania nanotubes $300^{\circ} \mathrm{C}(\mathrm{N})$ and $500^{\circ} \mathrm{C}(\mathrm{N})$ show no obvious difference with the untreated nanotubes with respect to the diffraction peak positions of anatase phase, indicating the amount of nitrogen 
incorporated is too little to affect the crystal lattice.

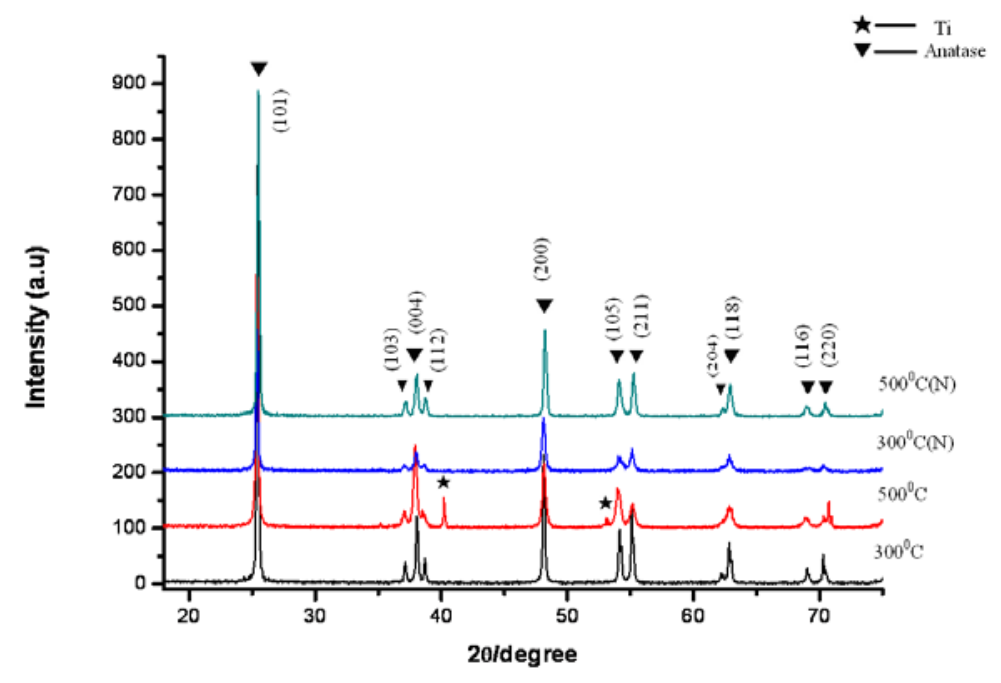

Figure 6.7. The XRD patterns of untreated and ammonium hydroxide (designated by $(\mathrm{N})$ ) $\mathrm{TiO}_{2}$ nanotubes

Figure 6.8 shows the Raman spectra of titania nanotubes with and without $\mathrm{NH}_{3}$ treatments after the annealing at $500^{\circ} \mathrm{C}$. The baseline with no peaks corresponds to the $\mathrm{TiO}_{2}$ foil. In both non-treated and $\mathrm{NH}_{4} \mathrm{OH}$-treated cases, the nanotubes show the typical peaks at 400,515 , and $640 \mathrm{~cm}^{-1}$ were all identified as $\mathrm{B}_{1 \mathrm{~g}}(\mathrm{~A}), \mathrm{A}_{1 \mathrm{~g}} / \mathrm{B}_{1 \mathrm{~g}}(\mathrm{~A})$, and $\mathrm{E}_{\mathrm{g}}(\mathrm{A})$ vibration modes of anatase phase ${ }^{83}$, respectively. From the spectra of $\mathrm{NH}_{3}$-treated nanotubes, we hardly can find the vibromode of titanium nitride, maybe this is because the incorporation of nitrogen is very little and did not introduce obvious changes in crystal lattice, in accordance with XRD analysis. 


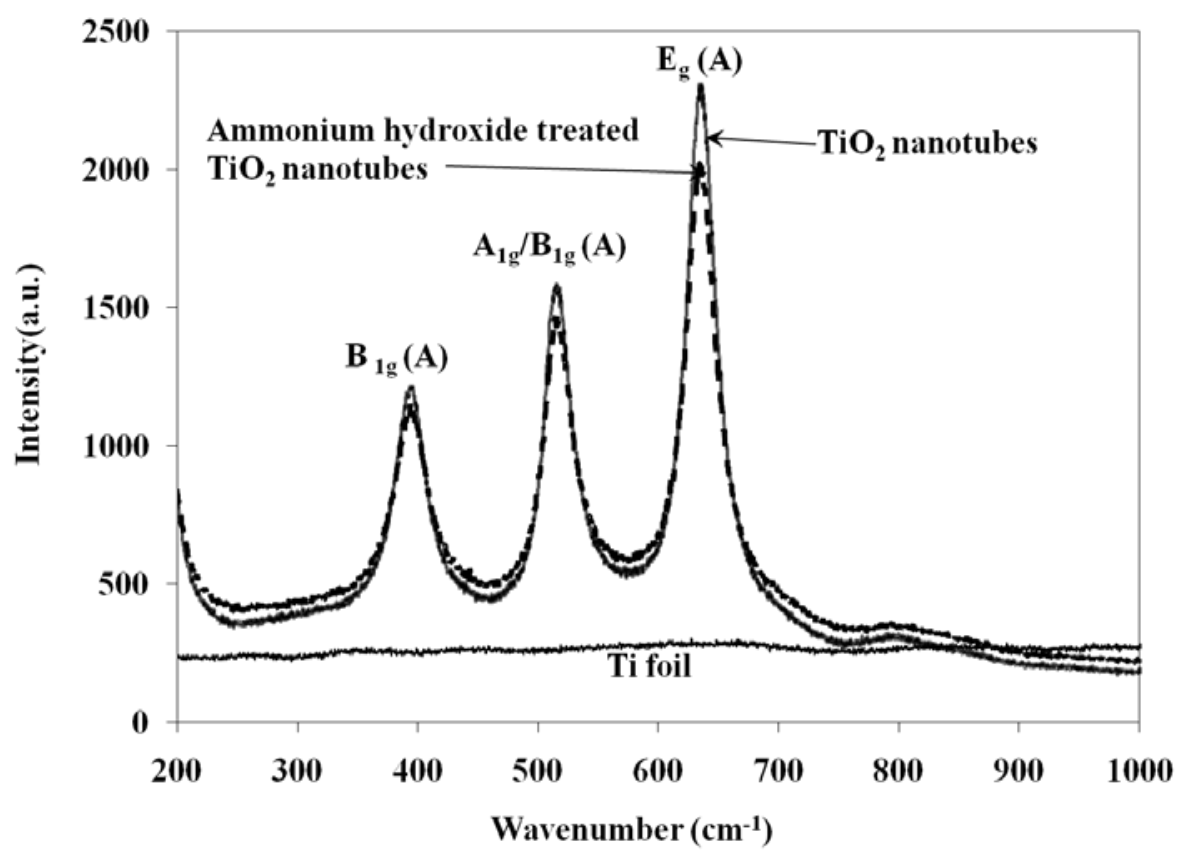

Figure 6.8. The Raman spectroscopy of the Ti foil, untreated and ammonium hydroxidetreated $\mathrm{TiO}_{2}$ nanotubes. The A in parenthesis stands for anatase.

The high resolution TEM and diffraction analysis of the $\mathrm{NH}_{4} \mathrm{OH}$-treated and annealed samples revealed a structural order similar to anatase phase. The bright field image shown in Figure 6.9 indicates the presence of structural defects in the nanoparticles. In addition, the diffraction analysis confirms that the dominant phase is anatase by the presence of (101) and (103) planes in the diffraction pattern (inset in Figure 6.9). The confirmation of anatase phase is quite interesting because the initial evidence based on the particle morphology suggests that the particles could be rutile phase. Sreekantan et al. ${ }^{84}$ reported the disintegration of the nanotube arrays into rutile particles after calcinations above $600^{\circ} \mathrm{C}$. Li et al. ${ }^{85}$ also reported similar phenomenon in $\mathrm{TiO}_{2}$ nanotubes. Dong et al ${ }^{86}$ concluded that nitrogen doping could facilitate the anatase-rutile phase transition at the annealing temperature of $500{ }^{\circ} \mathrm{C}$. In general, it is believed that rutile phase can not be stable in nanotubular morphology of $\mathrm{TiO}_{2}$ due to fact that the minimum nuclei size of rutile is usually larger than the wall thickness of nanotubes. ${ }^{87}$ Therefore, overall conclusion was that the formation of rutile phase is associated with the disintegration of nanotubes. 


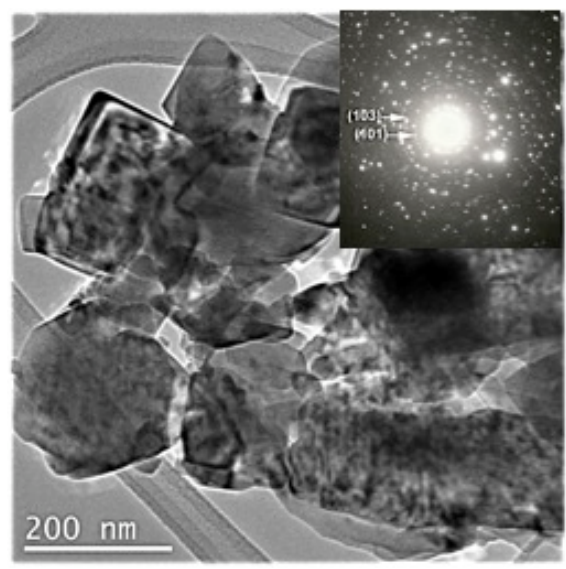

Figure 6.9. Bright field TEM images of nanoparticles after the collapse of $\mathrm{TiO}_{2}$ nanotubes by $\mathrm{NH}_{3}$-treatment and annealing at $500^{\circ} \mathrm{C}$. The inset shows the diffraction pattern of particles and indicates that the crystal structure is anatase.

\subsubsection{Discussion}

During the process of amorphous to anatase transformation in annealing the asanodized nanotubes, the length and average diameter of the nanotubes had no discernible changes. It has been suggested that the initial crystallization of anatase took place both in the nanotubes and in the nanotube-foil interface at almost the same temperature. ${ }^{88}$ In this process, some of the closely spaced anatase crystallites coalesced to form larger crystallites. 


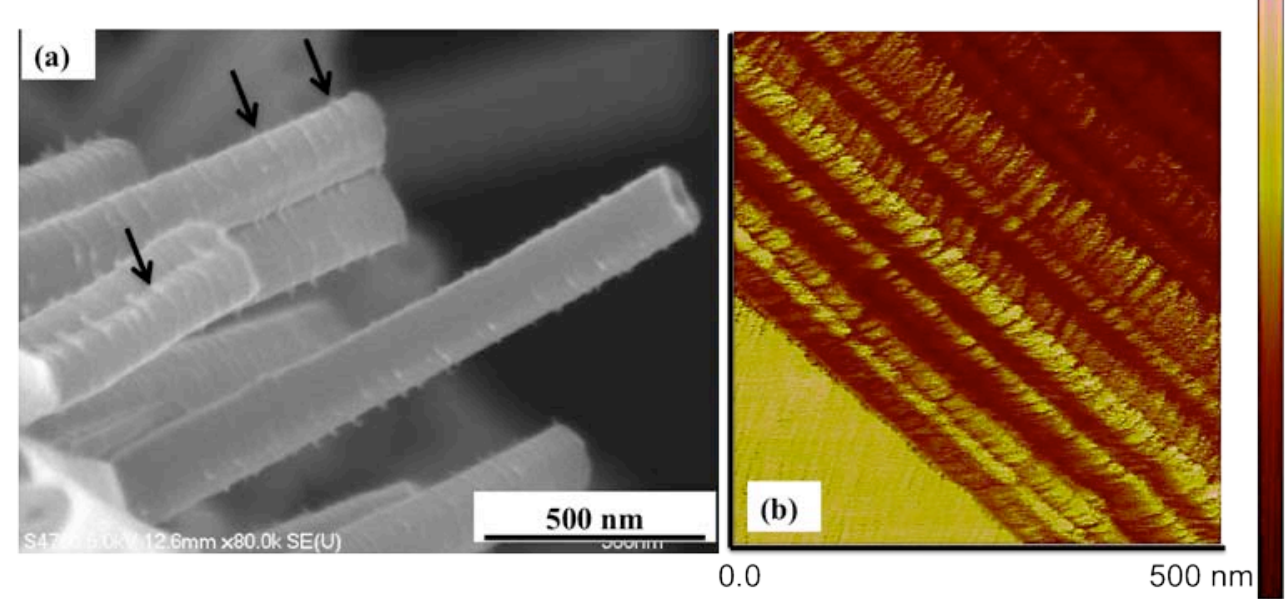

Figure 6.10. (a) The as-synthesized $\mathrm{TiO}_{2}$ nanotubes show imperfections on the surface

(marked by the arrows). (b) The elastic modulus map of $\mathrm{TiO}_{2}$ nanotubes before immersion in the ammonia hydroxyl media is shown. The dark brownish color indicates areas of high stiffness and areas of bright yellowish correspond to low stiffness values. The scanned area is $500 \mathrm{~nm}$ and the color key represents qualitative comparison of the modulus.

We found that it was difficult to incorporate nitrogen in the $\mathrm{TiO}_{2}$ lattice by a simple $\mathrm{NH}_{4} \mathrm{OH}$ treatment of $\mathrm{TiO}_{2}$ nanotubes. From the XRD spectra we hardly could find the vibromode of titanium nitride, and concluded that this was because the incorporation of nitrogen was very little and did not introduce obvious changes in crystal lattice. In addition, since the atomic radius of nitrogen is a little bigger than that of oxygen, the nitrogen doping should have led to compressive stress and eventually the broadening of vibrating modes. Such effect had been observed in phosphorus doped titania. ${ }^{89}$ A possible effect of $\mathrm{NH}_{4} \mathrm{OH}$ treatment of $\mathrm{TiO}_{2}$ nanotubes is the incorporation of nitrogen in surface roughnesses and weakening of the nanotubes. ${ }^{90}$ The nanotubes are in fact 'nanopits' that are formed on Ti foils and therefore should inheret the grain boundaries and defects that already exist in the foils. In addition, the nanotubes shown in Figure 6.1a and 6.10a exhibit surface roughness, which can be potential areas of chemical etching. The evidence of such weak boundaries is shown in Figure 6.10, which maps the stiffness of nanotubes. The Peak Force Tapping ${ }^{\odot}$ (PFT) mode of an atomic force microscopy 
(AFM) system was used to image the relative stiffness variation along the length of nanotubes. In PFT mode, the cantilever tapes the surface and makes very small deformation indents (less than $2 \mathrm{~nm}$ ). The maximum force required to achieve this was recorded and used to calculate the stiffness based on the unloading curves. The presence of high and low stiffness areas along the length of nanotubes indicates that these areas could potentially be the sites of misorientation regimes divided by grain boundaries.

Based on the above observations, we propose the following model (Figure 6.11) for the structural instabilities in $\mathrm{NH}_{4} \mathrm{OH}$-treated $\mathrm{TiO}_{2}$ nanotubes. The treatment of nanotube in $\mathrm{NH}_{4} \mathrm{OH}$ solution and subsequent calcination at $500^{\circ} \mathrm{C}$ results in structural transformation to anatase nanoparticles and the associated volumetric change results in high stress concentrations at the surface roughness areas. The nanotubes barely maintain their tubular morphology after $30 \mathrm{~min}$ of calcination. After this, the nanotubes will be collapsed into nanoparticles while the transformation to anatase happens simultaneously.

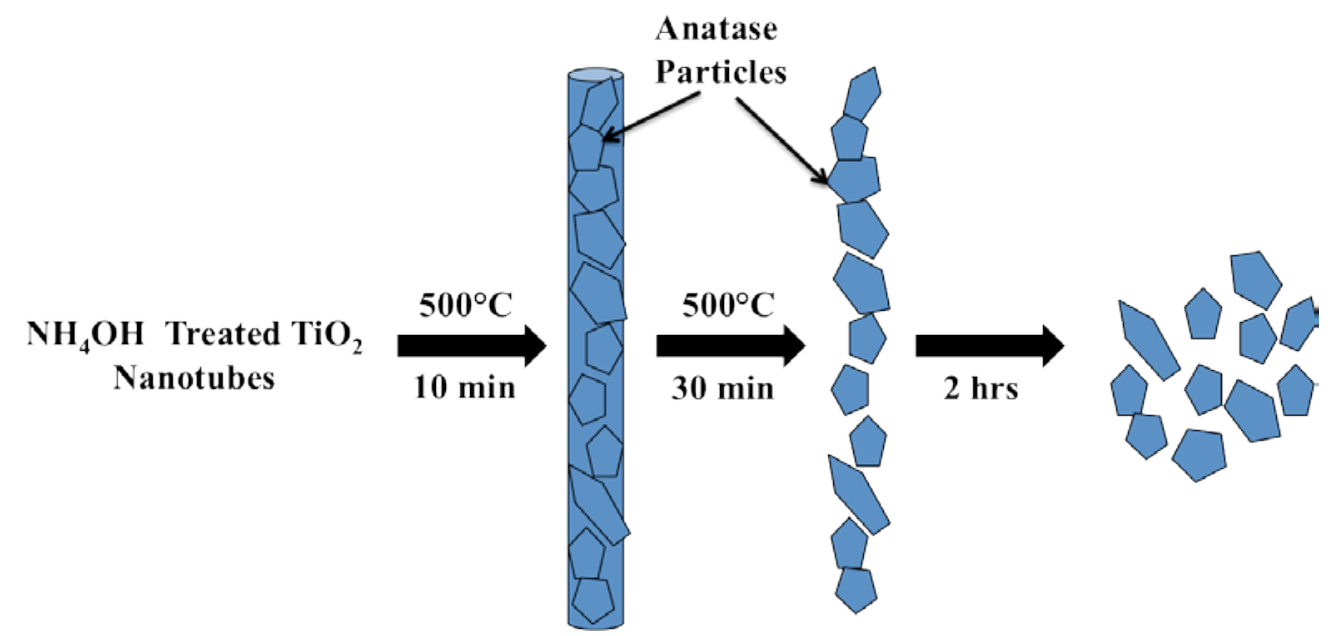

Figure 6.11. A schematic of the observed structural instability in $\mathrm{TiO}_{2}$ nanotubes treated with $\mathrm{NH}_{4} \mathrm{OH}$ and calcinated at $500^{\circ} \mathrm{C}$. The surface roughness is shown in the larger scale than the nanotube dimension. The nanotube disintegrated into anatase particles after 10 and 30 min of calcination but maintained the overall configuration of nanotube. After $2 \mathrm{hr}$ the nanotubes collapsed into nanoparticles.

\subsubsection{Conclusions}

$\mathrm{TiO}_{2}$ nanotubes subjected to treatment with ammonium hydroxide $\left(\mathrm{NH}_{4} \mathrm{OH}\right)$ solution 
followed by calcination at 500C exhibited an unexpected structural instability by collapsing into nanoparticles. High-resolution TEM, Raman spectroscopy, XRD, and AFM were used to understand the nature of structural collapse in the $\mathrm{NH}_{4} \mathrm{OH}$ treated $\mathrm{TiO}_{2}$ nanotubes. As opposed to the common belief that the transformation to rutile is the major reason for the collapse of the anatase nanotubes, it was determined that volumetric change due to phase transformation (amorphous $\rightarrow$ anatase) and surface roughness weakening due to chemical etching had the key role during the collapse of $\mathrm{NH}_{4} \mathrm{OH}$ treated $\mathrm{TiO}_{2}$ nanotubes.

\section{Acknowledgment}

This work was financially supported by the Multiscale Technologies Institute (MuSTI) at Michigan Technological University. 


\title{
6.2. A Real Time Observation of Phase Transition of Anatase $\mathrm{TiO}_{2}$ Nanotubes Into Rutile Particles by In-Situ Joule Heating Inside Transmission Electron Microscope
}

Anjana Asthana, Tolou Shokuhfar ${ }^{5}$, Qi Gao, Patricia A. Heiden, Craig Friedrich, Reza. S. Yassar

Advanced Science Letters, 3 (2010), 557

\begin{abstract}
We report here, the in-situ Joule heating induced phase transformation of anatase $\mathrm{TiO}_{2}$ nanotube into rutile particles inside a high-resolution transmission electron microscope (TEM) equipped with a scanning tunneling microscopy (STM) probe. Under the bias heating of $10 \mathrm{~V}$, the anatase $\mathrm{TiO}_{2}$ nanotube breaks into ultrafine anatase particles of average size between $5-10 \mathrm{~nm}$. On further increasing the bias voltage to $20 \mathrm{~V}$, the nanoclusters of anatase particles become prone to solid state reaction and are grown into stable rutile particles of size ranging between $20-25 \mathrm{~nm}$. The formation of rutile particles was confirmed by the observation of diffraction pattern and high resolution imaging of these particles, which corresponds to the tetragonal $\mathrm{TiO}_{2}$ rutile phase $(\mathrm{a}=4.59 \AA, \mathrm{c}=$ $\left.2.95 \AA, P 4_{2} / \mathrm{mnm}\right)$.
\end{abstract}

Keywords: $\mathrm{TiO}_{2}$ nanotubes, in-situ STM-TEM, Joule heating, Bias heating, Rutile particles, Anatase nanotube.

5 My main contribution to this publication was synthesizing the nanotubes, assisting some experimental work and discussions. 


\subsubsection{Introduction}

Titania $\left(\mathrm{TiO}_{2}\right)$ has been widely studied because of its many useful optical, electrical and photocatalytic properties, which depend on the crystalline structure of $\mathrm{TiO}_{2}{ }^{91}$ Technological applications have been proposed for $\mathrm{TiO}_{2}$ in bulk and in thin films forms. The $\mathrm{TiO}_{2}$ properties depend on phase composition, microstructure, crystallinity and chemical composition, which can be modified by preparation techniques, thermal treatments and metal ion doping. ${ }^{92}$

Titania crystallizes in three natural phases: brookite (orthorhombic), anatase (tetragonal) and rutile (tetragonal). The brookite and anatase crystalline phases, which are stable at low temperatures, transform into rutile when the sample is calcined at high temperature. ${ }^{93}$ It has been demonstrated that some properties of $\mathrm{TiO}_{2}$ are very sensitive to its structure. Since anatase phase is chemically and optically active, it is suitable for catalysts and supports. ${ }^{94}$ Usually, the anatase phase can be transformed into rutile via post-annealing, because anatase is thermodynamically unstable. This anatase-to-rutile phase transition, which is dependent upon annealing temperature, induces the variation of the optical properties in $\mathrm{TiO}_{2}$ films. Therefore, the post-annealing should be a controlled process in order to make desirable properties of a $\mathrm{TiO}_{2}$ film for various applications.

$\mathrm{n}$ vielv of the above, under the present investigations, we have taken a new approach to synthesize the rutile nanoparticles. Here, we report the phase transition of anatase $\mathrm{TiO}_{2}$ nanotube into nano-meter size rutile particles via in-situ Joule heating experiments inside a high resolution transmission electron microscope (TEM). The Joule heating experiment was carried out by using a special scanning tunneling microscopy holder from "Nanofactory Instruments" in a JEM 4000FX TEM, operated at 200kV.

\subsubsection{Results and Discussion}

The structural investigations of $\mathrm{TiO}_{2}$ nanotube samples were carried out during the in-situ TEM experiment. Fig. 6.12a is the low magnification bright field image of the assynthesized $\mathrm{TiO}_{2}$ nanotube. The general morphology shows that the as-synthesized $\mathrm{TiO}_{2}$ 
nanotubes are hollow and closed ended. The nanotubes have an average outer diameter of $100 \mathrm{~nm}$ and inner diameter of $\sim 50 \mathrm{~nm}$ and the wall thickness of the tubes are not uniform. The corresponding diffraction pattern (inset in Fig. 6.12a) and the high resolution transmission electron microscopy (HRTEM) image (Fig. 6.12b) clearly show that the tubular structures are amorphous.

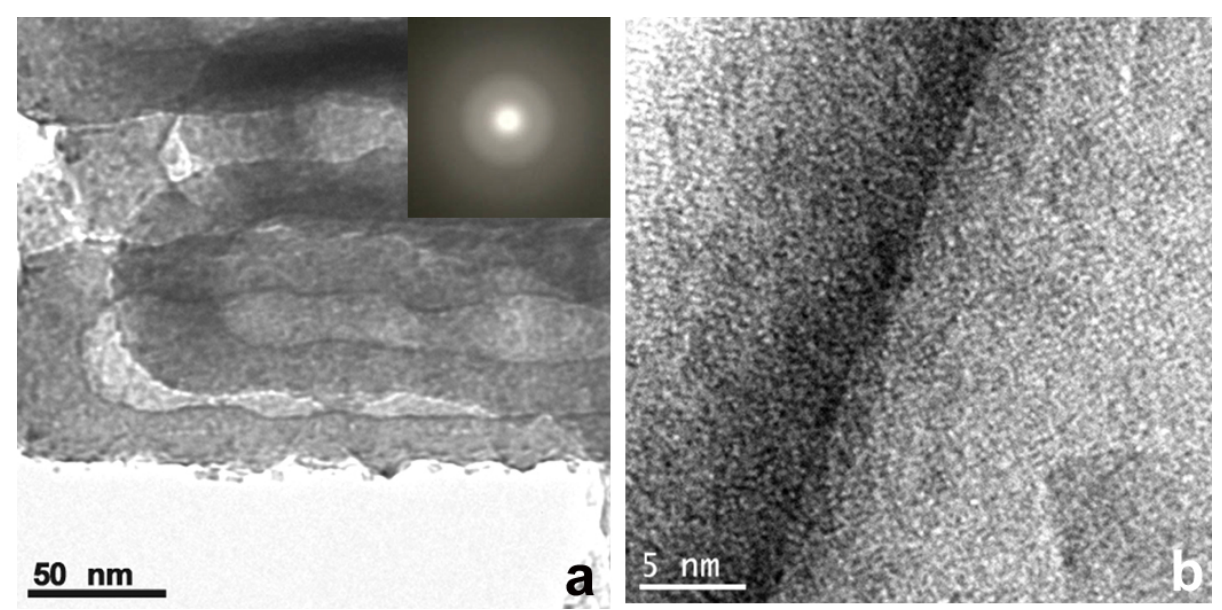

Figure 6.12. (a) TEM bright field (BF) image of the as-synthesized $\mathrm{TiO}_{2}$ nanotubes and its corresponding electron diffraction pattern (inset) and (b) the high resolution lattice image.

The as-synthesized $\mathrm{TiO}_{2}$ nanotube is transformed into crystalline anatase phase via post-annealing it at $450^{\circ} \mathrm{C}$ for $3 \mathrm{~h}$. Fig. $6.13 \mathrm{a}$ shows an over all view of the anatase titania nanotube, depicting a large number of tubular material with uniform size distribution. The tubes are hollow, open ended with an average inner and outer diameter of around $25 \mathrm{~nm}$ and $70 \mathrm{~nm}$ and the length range from several tens to several hundreds of nanometers. The corresponding diffraction pattern is shown in the inset (Fig. 6.13a), depicting the anatase titania ( $\left.a=0.378 \mathrm{~nm}, c=0.9513 \mathrm{~nm}, \mathrm{I}_{1} / \mathrm{amd}\right)$. The high resolution transmission electron microscopy (HRTEM) image (Fig. 6.13b) taken from a single nanotube (inset of Fig. 6.13b) shows that the nanotubes are well crystalline. The lattice fringe spacing of the walls of the nanotubes is estimated to be $\sim 0.353 \mathrm{~nm}$, corresponding to the interplanar distance of the (101) plane in the anatase phase. 

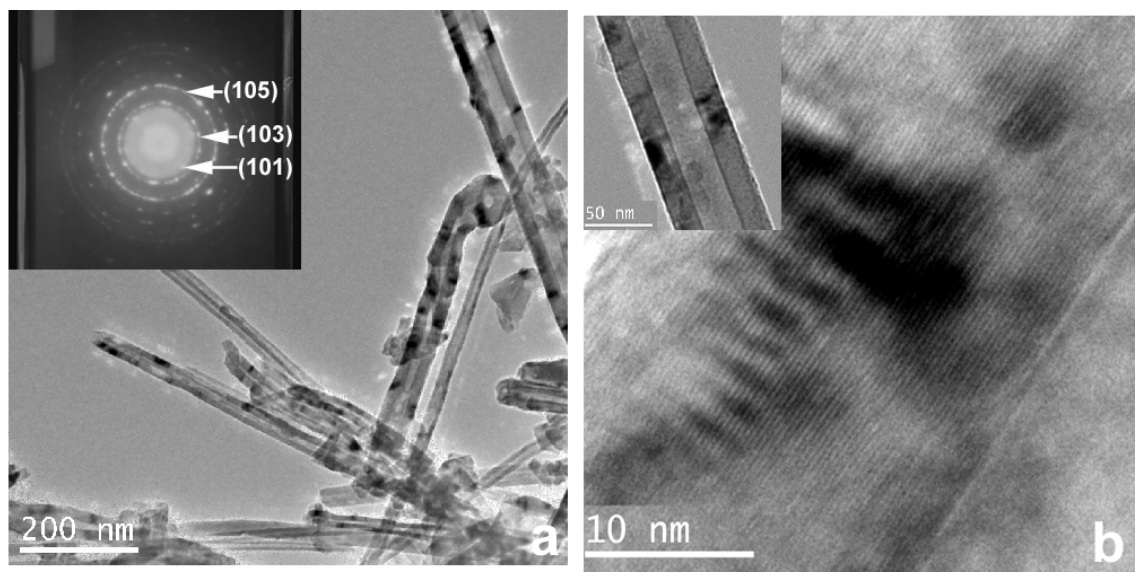

Figure 6.13. (a) An over all view of the anatase titania nanotube and the corresponding diffraction pattern (inset) and (b) the high resolution lattice image from a single anatase nanotube (inset).

In order to have a good electrical contact of the sample with tungsten tip, for in-situ electrical measurement, an individual $\mathrm{TiO}_{2}$ nanotubes were attached to the electromechanically etched gold tip by tungsten deposition using the focused ion beam (FIB) technique. The different steps of the sample preparation are shown in Figs. 6.14 (ac), in which a single nanotube is picked up by the FIB microprobe and attached to the tip of the tungsten wire. The gold tip with $\mathrm{TiO}_{2}$ nanotube was then loaded to the specimen holder and moved to its opposite conducting STM tip by a piezomanipulator. A schematic diagram of the experimental setup is shown in Fig. 6.14d. The Joule heating experiment was conducted by applying constant bias voltage, when the $\mathrm{TiO}_{2}$ nanotube is in contact with the STM tip. 

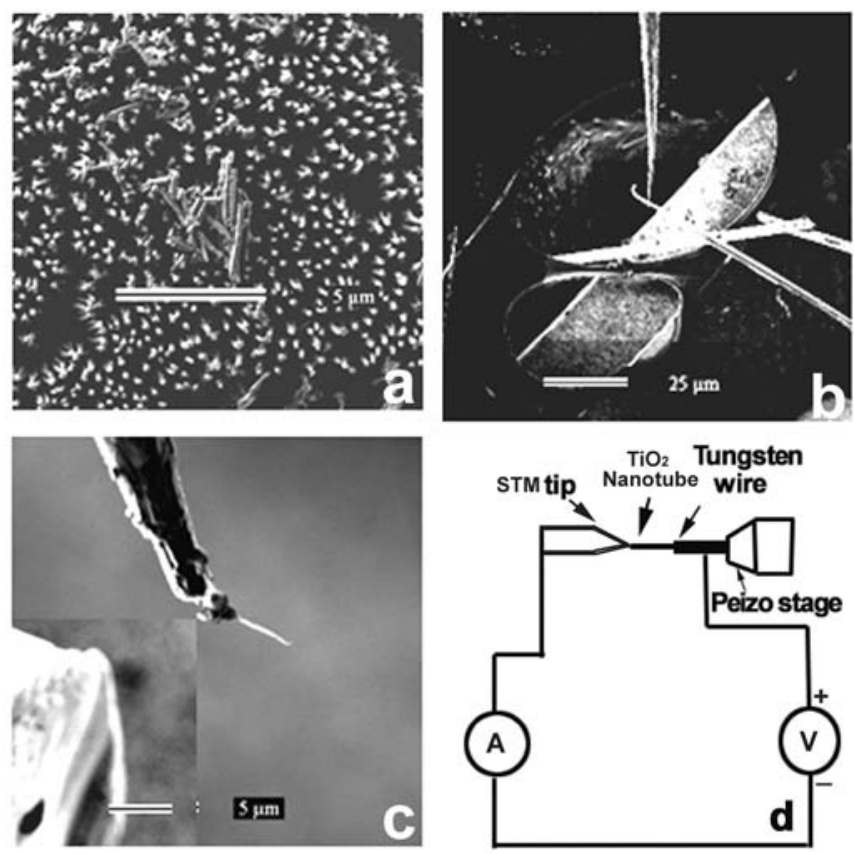

Figure 6.14. Images from the FIB system showing (a) the as grown $\mathrm{TiO}_{2}$ nanotube sample; (b) FIB probe attached with a single nanotube, dispersed on the $\mathrm{Cu}$ mesh (c) the FIB probe with a nanotube approaching the tip of the tungsten wire and (d) Schematic of the experimental setup for current - voltage measurement.

Fig. 6.15a shows a $\mathrm{TiO}_{2}$ nanotube contacted in both ends and free standing in high vacuum $\left(10^{-6}\right.$ Torr), inside TEM. Under the bias heating of $10 \mathrm{~V}$, the current, $I$, starts to increase gradually with time, $t$, and reaches a maximum value of $12 \mu \mathrm{A}$ in a period of 180 sec, as shown by the $I$ vs $t$ plot (Fig. 6.16). Figs. 6.15(b) shows the image of the $\mathrm{TiO}_{2}$ nanotubes undergone the heat treatment at constant bias voltage of $10 \mathrm{~V}$ for $180 \mathrm{sec}$. The analysis of the image (Fig. 6.15b) shows that the $\mathrm{TiO}_{2}$ nanotube still maintains its morphological shape, but is broken into ultrafine particles. 

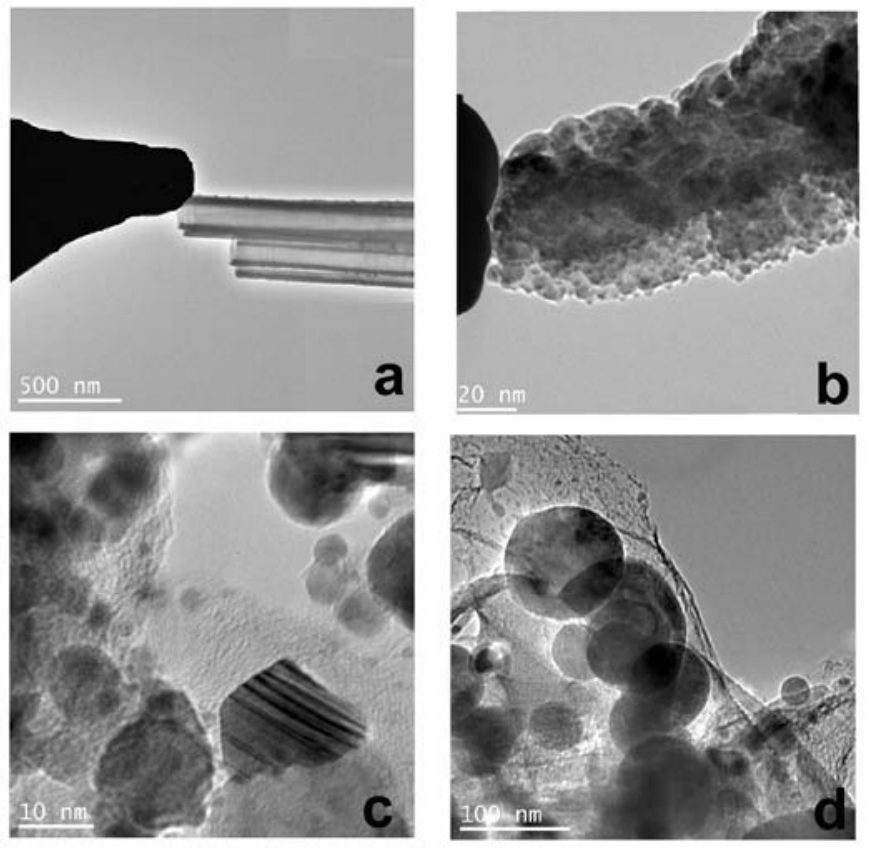

Figure 6.15. The $\mathrm{BF}$ image of the $\mathrm{TiO}_{2}$ nanotube (a) in contact with the STM tip (b) undergone the heat treatment at constant bias voltage of $10 \mathrm{~V}$ for $180 \mathrm{sec}$ (c) the bias heated at $20 \mathrm{~V}$ for $130 \mathrm{sec}$ (d) undergone the bias heating at $30 \mathrm{~V}$.

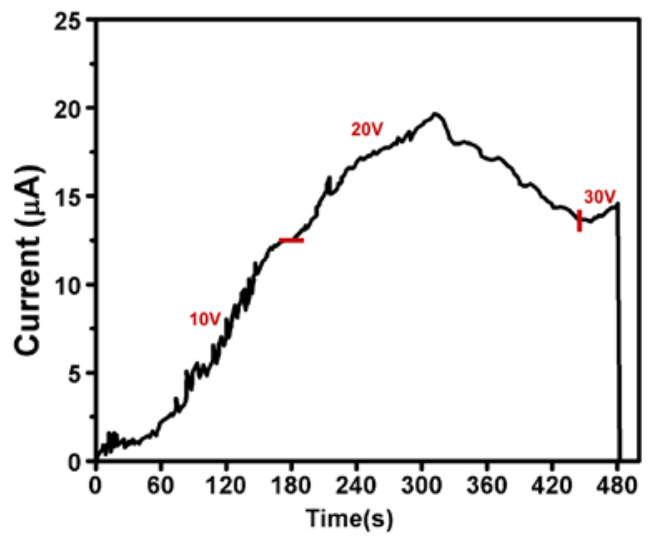

Figure 6.16. The current $(\mathrm{I})$ versus time ( $\mathrm{t}$ ) plot for the $\mathrm{TiO}_{2}$ nanotube under the different bias heating voltages, as marked in the plot.

From the previous reports ${ }^{95}$, it is known that the thermodynamic stability of $\mathrm{TiO}_{2}$ 
particle is size dependent, each phase has a particle size distribution and at particle diameters less than $11 \mathrm{~nm}$, the anatase is the most stable phase. The particle size in the anatase phase can range from $0.1 \mathrm{~nm}$ to $11 \mathrm{~nm}$. ${ }^{96}$ Therefore, from the morphology of the particle size, it can be said that at this stage the nanotube is mainly comprised of ultrafine anatase particles. The diffraction pattern and the lattice image taken from this stage of $\mathrm{TiO}_{2}$ nanotube also confirm the presence of anatase phase (Fig. 6.17a). On further increasing the bias voltage to $20 \mathrm{~V}$, it was observed that the current increased drastically to a maximum value of $20 \mu \mathrm{A}$ in a time period of $130 \mathrm{sec}$ and then starts to decrease gradually with the further breaking of the nanotube and at the same time nanometer diameter titania becomes prone to solid state reaction and grown into bigger crystal of size 20-25 nm (as shown in the image of Fig. 6.15c), which is comparable to the particle size of around $30-35 \mathrm{~nm}$ for the rutile phase to become stable.This leads to the alteration of phase stabilities and ultimately the anatase phase is converted into stable rutile phase. Furthermore, the presence of the rutile phase is also confirmed by the diffraction pattern and high resolution image taken from these particles as shown in Fig. 6.17b, which corresponds to the tetragonal $\mathrm{TiO}_{2}$ rutile phase $\left(\mathrm{a}=4.59 \AA\right.$, $\left.\mathrm{c}=2.95 \AA, \mathrm{P}_{2} / \mathrm{mnm}\right)$.

On increasing the bias heating voltage to $30 \mathrm{~V}$, the current is increased slightly from $13.75 \mu \mathrm{A}$ to $15 \mu \mathrm{A}$ in a time period of $30 \mathrm{sec}$. Due to this increase in current at this stage, some of the rutile particles form large sized agglomerations of around 100-200 nm and finally the nanotube structure is collapsed. The diffraction pattern of these agglomerations show that they are crystalline (Fig. 6.17c) 

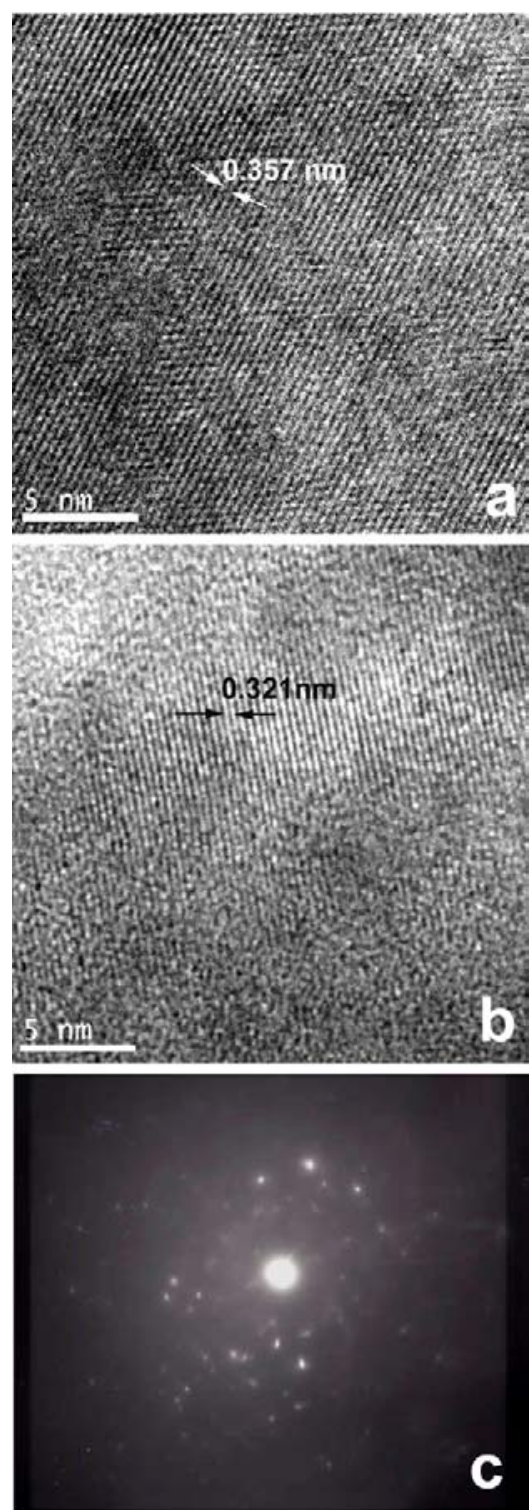

Figure 6.17. (a) The high resolution lattice image and the corresponding diffraction pattern (inset) taken from $\mathrm{TiO}_{2}$ nanotube undergone a bias heating at $10 \mathrm{~V}$ (b) the high resolution lattice image of the nanotube bias heated at $20 \mathrm{~V}$ and the corresponding diffraction pattern (inset) (c) The diffraction pattern from the agglomeration formed at the bias heating of $30 \mathrm{~V}$.

ndel the present investigations, it was observed that via bias heating through STM- TEM system the anatase $\mathrm{TiO}_{2}$ nanotube is transformed into stable rutile particles. 
It can be said that when the $\mathrm{TiO}_{2}$ nanotube is subjected to a bias voltage of $10 \mathrm{~V}$ for about $180 \mathrm{sec}$, the nanotube is resistively heated up to elevated temperatures, which reduces the total resistance and a high current of $12 \mu \mathrm{A}$ could be detected. On further increasing the bias voltage to $20 \mathrm{~V}$, a high current of $20 \mu \mathrm{A}$ flows through the nanotube and temperature of the system is further increased. The nanometer sized anatase particles, having high surface area become prone to solid state sintering, which leads to grain growth and transformation to stable rutile particles of average size between 20-25 nm. Eventually there is complete collapse of the structure, when the bias heating voltage is further increased to $30 \mathrm{~V}$. At this stage, the rutile particles already under the heated condition, forms agglomeration of size 100-200 nm with slight increase of current of $\sim 2 \mu \mathrm{A}$. Such processes are more pronounced during phase transformations, when bond breaking and enhanced mass transport take place, which can lead to grain growth and densification. ${ }^{97}$ Also in the case of anatase to rutile transformation, the mechanism responsible for the transformation are most likely the spatial disturbance of the oxygen ion framework and the shifting of the majority of $\mathrm{Ti}^{4+}$ ions by breaking two of the six Ti-O bonds to form new bonds ${ }^{98}$. In the present case, $\mathrm{TiO}_{2}$ nanotube heated at high bias voltage of $20 \mathrm{~V}$, obtains sufficient energy to disturb the oxygen ion framework and form new bonds to form the rutile phase.

\subsubsection{Experimental Section}

The in-situ Joule heating experiment was conducted in a high resolution transmission electron microscope (TEM) using an STM-TEM system from the "Nanofactory Instruments". The STM-TEM system provides a unique combination of transmission electron microscopy and scanning tunneling microscopy techniques, which are used simultaneously in one instrument for the full sample characterization. It consists of a STM equipped TEM sample holder, a controller and a PC with Nanofactory's data acquisition software. All the measurements were carried out on a single tilt STM-TEM holder in a JEM 4000FX TEM, operated at 200kV. The electrochemically etched gold wire with $\mathrm{TiO}_{2}$ nanotubes was attached to the piezo-driven movable part of the holder 
facing the fixed and sharp tungsten STM tip as its counter electrode and oriented perpendicular to the electron beam in TEM. In such an arrangement, atomic scale imaging and I-V measurement were carried out concurrently. The contact was made between the STM tip and the $\mathrm{TiO}_{2}$ nanotube by the precision movement of gold wire (with the sample) attached with the piezo-driven manipulator. Joule heating experiment was conducted by applying different bias voltages to the gold electrode with the nanotube sample at its tip, while the tungsten STM tip was grounded. Under the present investigations, $\mathrm{TiO}_{2}$ nanotube sample were synthesized by anodization of $\mathrm{TiO}_{2}$ foils, details of the synthesis process is reported elsewhere. ${ }^{99}$

\subsubsection{Conclusion}

In conclusion, we have shown, for the first time, the anatase nanotube dissociation and their phase transformation to rutile in real time. The anatase nanotubes under Joule heating dissociate to small particles $(\sim 10-20 \mathrm{~nm})$ of anatase at low bias voltage $(\leq 10 \mathrm{~V})$. At intermediate bias range, $10 \mathrm{~V}<$ bias $<20 \mathrm{~V}$, the anatase nanoparticles transform to rutile. The size of the rutile nanoparticles can be controlled by the applied bias voltage. Under the bias heating condition of $30 \mathrm{~V}$, the rutile particles agglomerate into large particles with sizes range up to $200 \mathrm{~nm}$. It is known that thermodynamic stability of different phases of $\mathrm{TiO}_{2}$ is size dependent and thus by controlling the size of the particles, we can control the properties of the $\mathrm{TiO}_{2}$ used for various applications.

\section{Acknowledgment}

I would like to acknowledge the funding support through NSF-DMR grant no. 0820884 and NSF-CMMI grant no. 092681 


\title{
Chapter 7
}

\section{Droplet Wettability Changes of Titania Nanotube Surfaces Due to Annealing and Aging}

\author{
Dong Hwan Shin, Tolou Shokuhfar ${ }^{6}$, Chang Kyoung Choi, Seong-Hyuk Lee, Craig \\ Friedrich
}

\begin{abstract}
This study examines the effect of annealing and aging on the wettability of titania nanotube (TNT) surfaces fabricated by anodization. The fabricated TNTs are $60 \sim 130 \mathrm{~nm}$ inner diameter and 7 10 microns high. One-microliter water droplets were used to define the wettability of the TNT surfaces by measuring the contact angles. A digital image analysis algorithm was developed to obtain contact angles, contact radii, and center-heights of the droplets on the TNT surfaces. Bare titanium foil is inherently less hydrophilic with approximately 60 80 degree contact angle. The as-anodized TNT surfaces are more hydrophilic and annealing further increases this hydrophilic property. Furthermore, it was found that the TNT surface became more hydrophobic when aged in air over a period of 3 months. This work can provide guidelines to better specify the wettability characteristics of titanium orthopedic implants.
\end{abstract}

Keywords: Titania nanotube, Wettability, Hydrophilic, Hydrophobic, Contact angle, Aging effect

${ }^{6}$ My contributions to this work includes: $\mathrm{TiO}_{2}$ nanotubes (TNTs), synthesize, annealing and crystallization, SEM and TEM characterizations, chemical analysis, FTIR analysis of surface chemistry for both fresh and aged TNTs, the wettability study comparison and conclusions between fresh and aged TNTs based on FTIR analysis. 


\subsection{Introduction}

A thin film sprayed with specially treated titanium dioxide $\left(\mathrm{TiO}_{2}\right)$ can be applied to a window to act as an anti-fogging agent and is also heat tolerable up to $400{ }^{\circ} \mathrm{C}$. When dust falls on a titanium-dioxide-treated window or surface, clean water can easily remove it. The hydrophilic nature of titanium dioxide, ${ }^{100}$ coupled with gravity, will enable dust particles and bio slime to be swept away by a water stream, making the product selfcleaning. ${ }^{101}$

Applications of self-organized porous media, for example proteins, target the use of the high order or the high surface area of the structures for catalysis. ${ }^{102}$ Another potential application of porous media is based on controlling the surface wettability and this has attracted attention in the field of biological systems. Products are available that are made of titanium and have the capability to kill bacteria and disassociate organic gases by photocatalysis. ${ }^{103}$ Titanium and its alloys are widely used as biocompatible materials, such as human body implants. It is therefore important to control and modify the surface topography and tailor its wetting characteristics.

Titanium is widely used as a biocompatible material; therefore a change of surface topography and wetting behavior is important. Recently, cell adhesion, growth and proliferation have received a great deal of attention in the fields of micro-fluidics and in micro-system applications including mechanical, electronic and biological engineering. ${ }^{104}$ For biological systems, the nature of hydrophobic and hydrophilic forces plays a key role in protein adsorption and cellular adhesion. ${ }^{105}$ The wettability of cell surfaces affects not only adsorption of DNA and proteins but also cell adhesion, surface morphology, and structure. The characteristics of a cell's adhesion and growth vary with its contacting surface conditions. ${ }^{106}$ Some cells prefer hydrophilic surfaces, while other cells have the characteristic of attraction to hydrophobic surfaces. ${ }^{107}$

The simplest way to express the wettability characteristics of surfaces is the contact angle of water droplets resting on the surface. Surface modifications are of ever increasing interest in a wide range of fields including water-repellent surfaces and bio- 
synthetic structures for mechanical and electronic devices. ${ }^{108}$

In the research reported here anodization with was used to fabricate TNT surfaces. The effect of annealing and aging on the wettability characteristics, including changes from hydrophilicity to super-hydrophilicity of the nanotubes were studied using contact angle analysis of droplets of de-ionized water on the surfaces. The timedependent changes of surface wettability were also analyzed for various TNT surfaces.

\subsection{Nanotube Fabrication}

$\mathrm{TiO}_{2}$ nanotubes were fabricated by anodization as shown in Fig. 7.1. Pure titanium foils (purity $>\sim 99.7 \%$ ) and titanium alloy foils (Ti-6Al-4V) were anodized in an electrochemical cell with an electrolyte containing ethylene glycol (EG) and ammonium fluoride $\left(\mathrm{NH}_{4} \mathrm{~F}, 48 \%\right.$ aqueous solution). Before anodization the titanium foils were cleaned with ethanol and acetone, then rinsed with de-ionized water, and dried with $\mathrm{N}_{2}$. The applied voltage, $\mathrm{NH}_{4} \mathrm{~F}$ concentration in $\mathrm{EG}$, and duration of anodizing were, 60VDC, $0.2 \mathrm{wt} \%$, and three hours, respectively.

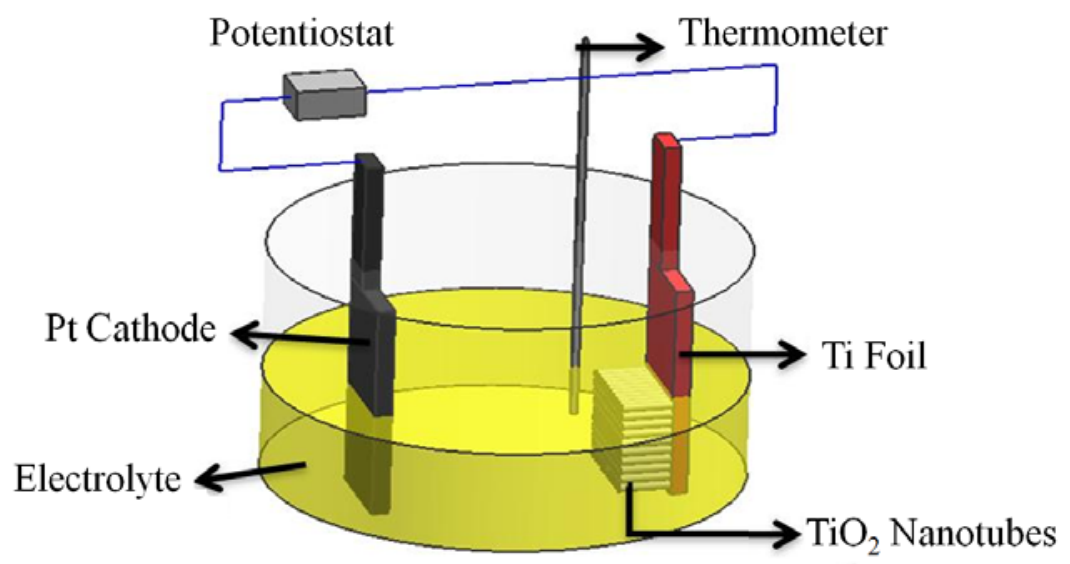

Figure 7.1. Electrochemical etching (anodization) setup for the $\mathrm{TiO}_{2}$ nanotube fabrication. The anodization setup consisted of a Ti foil, a Pt counter electrode, a voltage source, and a themometer. The nanotubes were etched on the Ti foil substrate. 
Platinum mesh was used as the counter electrode. After anodization, thermal annealing of the TNTs was performed in air at $450{ }^{\circ} \mathrm{C}$ for three hours with heating and cooling rates of $7.5^{\circ} \mathrm{C} / \mathrm{min}$. This annealing treatment produced an anatase phase to study its effects on the wettabillity of the nanotubes. All samples were tested after one day allowing the samples to become structurally stable. Scanning electron microscopy was used for the structural and morphological characterization of the titanium nanotubes, as shown in Fig. 7.2. The anodization resulted in vertically oriented $\mathrm{TiO}_{2}$ nanotube arrays. In general, the nanotubes had lengths between 7 10 microns and inner diameters ranging from $60 \sim 130 \mathrm{~nm}$. The morphology of the annealed crystalline nanotubes (Fig. 7.2c and d) compared with the as-synthesized nanotubes (Fig. 7.2a and b) did not show any significant difference. Transmission electron microscopy (TEM) images of the assynthesized and annealed nanotubes are shown in Fig. 7.3 (a) and (b), respectively. Fig. 7.3a shows a disordered atomic arrangement in the nanotubes confirmed by the diffuse diffraction pattern (upper right in Fig. 7.3a). The transition in atomic ordering to crystalline is evident in Fig. 7.3b and the corresponding high-resolution image and diffraction pattern shown in the upper right. 

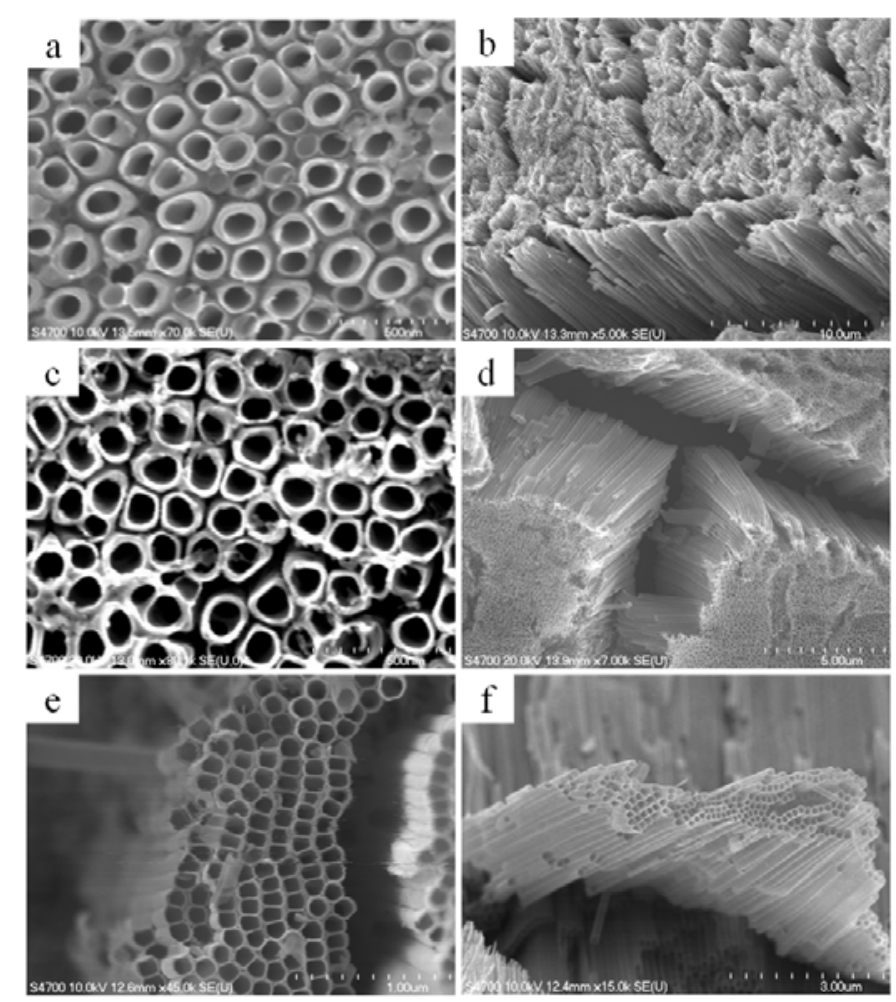

Figure 7.2. Titanium dioxide nanotubes prepared by Pt electrode $(\mathrm{a} \sim \mathrm{f})$. In general, titania nanotubes had lengths bewteen 7 10 microns and inner diameters ranging from 60 130 nm. (a) and (b) are amorphous, (c) and (d) are crystalline, (e) is as-sythesized, and (f) is annealed TNT. 
High Resolution

TEM image shows no sign of crystalline order

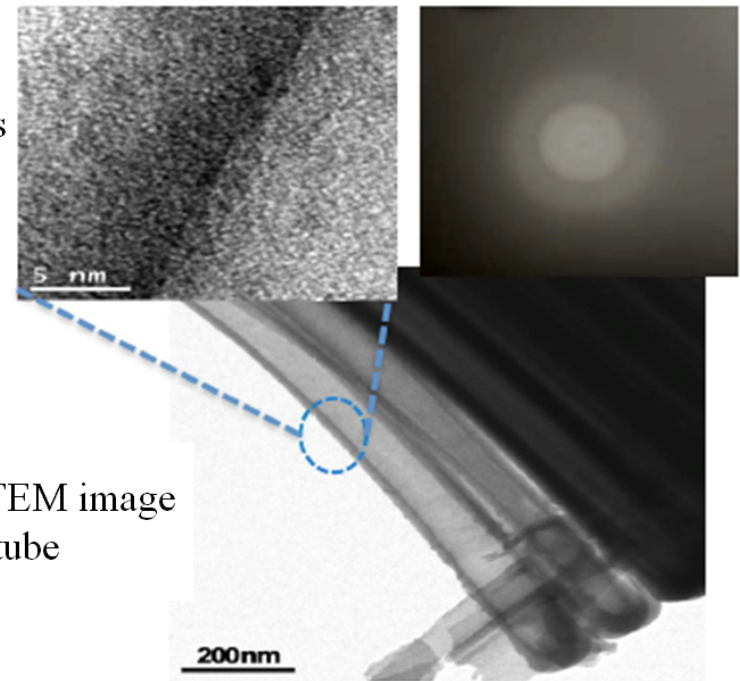

Electron diffraction pattern shows a fuzzy ring

Bright field TEM image of $\mathrm{TiO}_{2}$ nanotube

$200 \mathrm{~nm}$

(a)

High Resolution TEM image shows crystalline order

Bright field TEM image of $\mathrm{TiO}_{2}$ nanotube

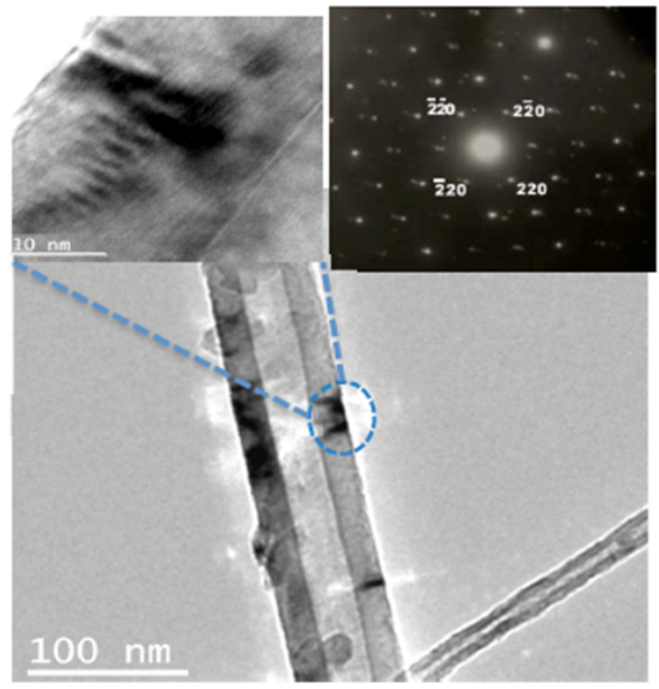

Electron

diffraction shows spot pattern of anatase phase

(b)

Figure 7.3. TEM images of TNT structured surfaces (a) as-sythesized nanotubes are amorphous, (b) annealed nanotubes are crystalline.

\subsection{Contact Angle Measurements}

Commercially pure and grade-5 (Ti6A14V) titanium alloy foils were used to fabricate $\mathrm{TiO}_{2}$ nanotubes. One-microliter of de-ionized (DI) water was slowly deposited 
on the surfaces by a screw syringe. The experiments were repeated five times for each measurement. The temperature and humidity were maintained at $24 \pm 0.5^{\circ} \mathrm{C}$ and $21 \pm$ 1\%, respectively. As shown in Fig. 7.4, the contact angle measurement system consisted of a halogen lamp (400W, 3M Overhead Projectors), a stage to help place the water drops, and a long distance microscope (Infinity $\mathrm{K} 2 / \mathrm{S}$ ) coupled to a CCD camera (PULNIX TM-1325CL). The motion was by an $x-y$ translation stage (Velmex AXY2509W1) on a lab jack (Thorlabs L200) which enabled x-y-z movement of the substrate. The camera was connected to a frame grabber (EPIX EL1DB) and the images were captured using EPIX XCAP software.

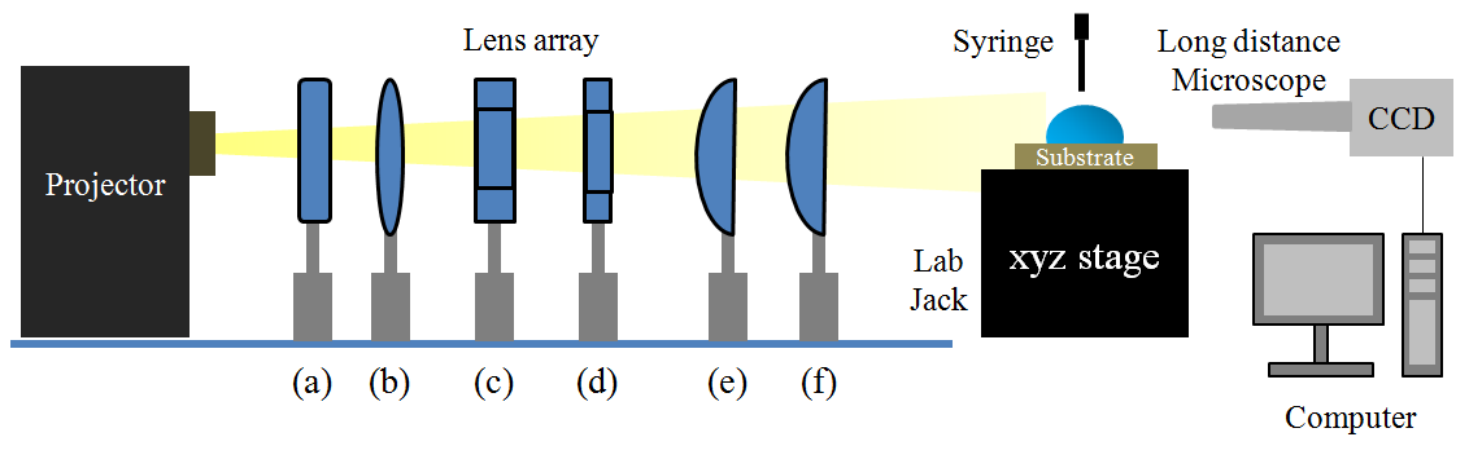

Figure 7.4. Schematic of the experimental setup. Lens array consisted of (a) diffuser, (b) bi-convex lens, (c) large aperture, (d) small aperture, (e) plano-convex (KPX079), and (f) plano-convex (KPX088).

Prior to the experiments, droplet deposition was repeatedly tested to ensure that the water droplets had consistent shapes. During the experiment, a droplet was dynamically recorded on an image capturing system via the CCD camera. A perpendicular view was also recorded to show that the droplets were a part of a spherical cap. To analytically determine if the shape of the droplets on TNT surfaces had a spherical cap, the Bond number, which is a dimensionless number expressing the ratio of gravitational force to surface tension force, was calculated according to, 


$$
B_{0}=\Delta \rho \cdot g \cdot R \cdot h_{0} / \sigma
$$

Where $\Delta \rho$ is the density difference between water and air, $g$ is the gravitational constant, $R$ is the contact line radius, $h_{0}$ is the initial height of the droplet, and $O$ is the air-water surface tension. ${ }^{109}$ The calculated Bond number was in range of 0.01-0.24 for all samples, indicating that surface tension dominated. It was confirmed that the shapes of the droplets were a part of a sphere.

Images of droplets on the surfaces were captured five to ten times for each case and the corresponding contact angles calculated. The code distinguished the triple point among the air, the droplet, and the substrate through Laplace's equation, which considers the mathematical balance between the surface tension and gravitational force using the Laplace curve and the droplet height. The code, which utilizes the Runge-Kutta method, was selected to obtain the drop profile and this code was validated with the numerical data published by Hartland and Hartley. ${ }^{110}$ This analysis method was used for this study because the Wilhelmy Plate method has severe limitations with regards to rough and porous substrates such as TNT surfaces. Also, goniometry is more approximate and the contact angles are dependent on the judgment of the observer so it was not considered for this work. In the present study, the uncertainty in the determination of the contact angles was within $10 \%$, considering all data samples for each experimental condition $(95 \%$ confidence level). Uncertainties of temperature and humidity were estimated to be $6 \%$.

\subsection{Results and Discussion}

Figure 7.5 (a) shows representative images of initial water droplets on bare Ti foil, pure TNT, and alloy TNT in as-synthesized and annealed conditions. The trend of the contact angle variation is illustrated in Fig. 7.5 (b). Three phenomena were observed. The first was the difference between the contact angles of the bare pure and bare alloy titanium foils. In the case of pure titanium, the contact angles were larger than the alloy. 

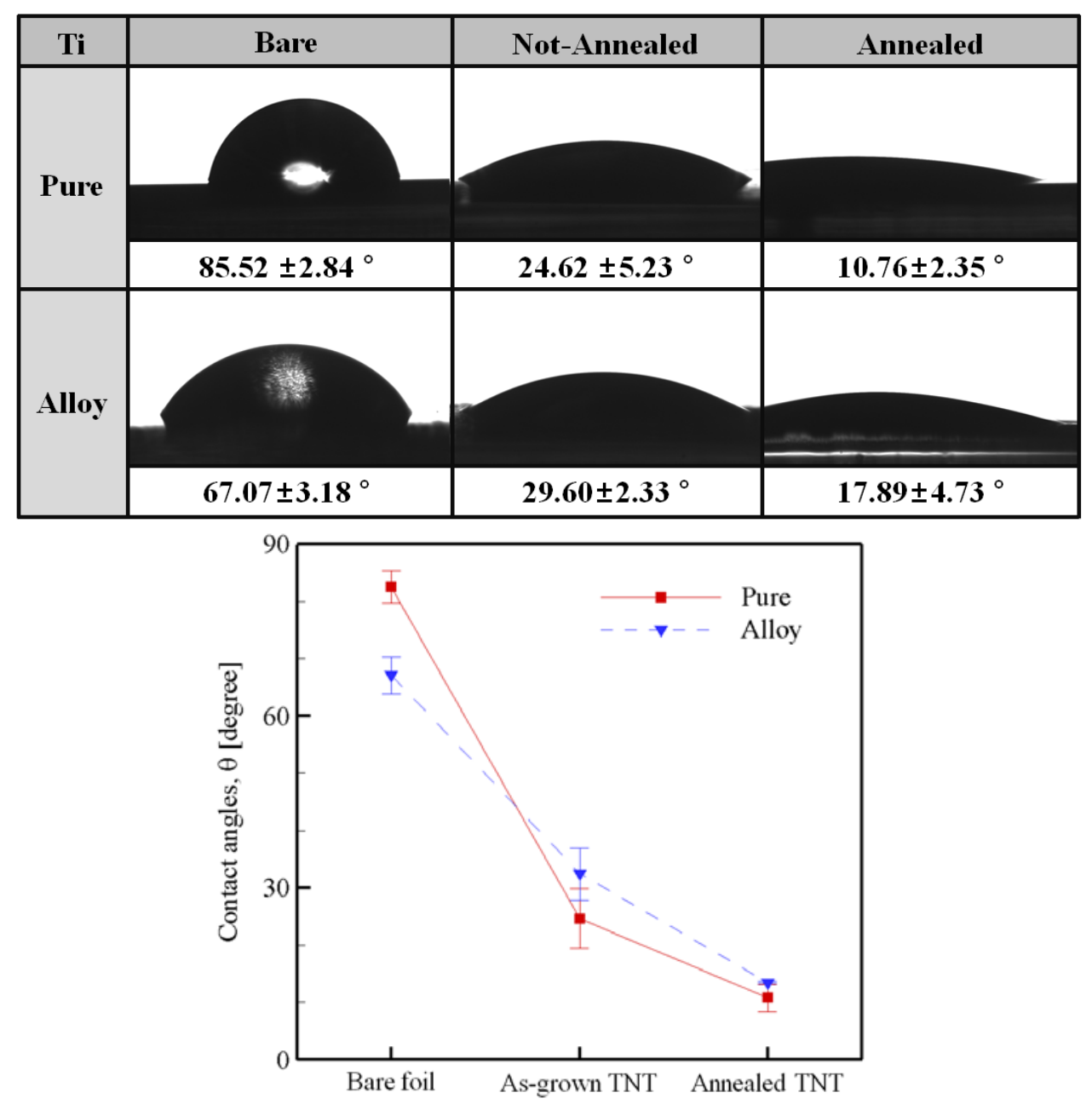

Figure 7.5. (above) Representative image comparison of the initial contact angles. Six samples in total were investigated. (below) Results of the contact angles on the surfaces. The TNT structures made the surface more hydrophilic and the annealing effect made the TNT surface super-hydrophilic. Contact angle differences were attributed to the surface properties, in particular the surface roughness, which could affect the initial contact angle of a water droplet on each titanium surface.

Second, it was found that the creation of TNT structures made the surface more hydrophilic. For all cases after the anodization process, the droplet contact angles decreased in the range of 55 70\% compared to the contact angles of each bare foil. Before conducting the experiments, we expected the water droplet contact angles to 
increase on the fabricated TNT surface because it was thought that the TNT surface would consist of $\mathrm{TiO}_{2}$ which has hydrophobic properties. ${ }^{111}$ However, the results were contradictory, the contact angles decreased for all cases. To explain this, the chemical equations were considered for the anodization process. In the solution, the ethylene glycol (EG) is a solvent so it does not participate in the chemical reaction. The ammonium fluoride can act as a catalyst and be ionized into the ammonium ion $\left(\mathrm{NH}^{+}\right)$ and the fluorine ion $\left(\mathrm{F}^{-}\right)$. Next, the water $\left(\mathrm{H}_{2} \mathrm{O}\right)$ can be ionized into hydrogen ions $\left(\mathrm{H}^{+}\right)$ and hydroxide $\left(\mathrm{OH}^{-}\right)$. Here, electrolytic dissociation of the $\mathrm{F}^{-}$is larger than the $\mathrm{OH}^{-}$so even though the ammonium fluoride participates in the chemical reaction as a catalyst, the product can be $\mathrm{Ti}(\mathrm{OH})_{4}$ rather than $\mathrm{TiF}_{4}$. Thus, there are two possible chemical reactions,

$$
\begin{gathered}
4 \mathrm{H}_{2} \mathrm{O}+\mathrm{Ti} \underset{\mathrm{HO}\left(\mathrm{NH}_{2}\right)_{2} \mathrm{OH}}{\longrightarrow} \mathrm{Ti}(\mathrm{OH})_{4}+2 \mathrm{H}_{2} \uparrow \\
4 \mathrm{H}_{2} \mathrm{O}+4 \mathrm{NH}_{4} \mathrm{~F}+\mathrm{Ti} \underset{\mathrm{HO}\left(\mathrm{CH}_{2}\right)_{2} \mathrm{OH}}{\longrightarrow} \\
\mathrm{Ti}(\mathrm{OH})_{4}+2 \mathrm{H}_{2} \uparrow+4 \mathrm{NH}_{4}^{+}+2 \mathrm{~F}_{2} \uparrow
\end{gathered}
$$

In (2), the ammonium fluoride acts as the catalyst. Accordingly the products are $\mathrm{Ti}(\mathrm{OH})_{4}$ and $\mathrm{H}_{2}$ vapor obtained on the cathode side. However, during the anodization, vapor was observed on both electrodes so reaction (2) was apparently not occurring. In (3), the ammonium fluoride participates in the chemical reaction. Here, $\mathrm{Ti}(\mathrm{OH})_{4}, \mathrm{H}_{2}, \mathrm{NH}_{4}{ }^{+}$, and $\mathrm{F}_{2}$ are obtained. From the viewpoint of the electrolytic dissociation ability between the $\mathrm{F}^{-}$ and the $\mathrm{OH}^{-}$, the $\mathrm{NH}_{4}{ }^{+}$remains in the form of an ion within the solution. The $\mathrm{F}_{2}$ vapor can be generated at the anode and the $\mathrm{H}_{2}$ vapor at the cathode. Thus, (3) better described the experimental situation. Consequently, after anodization the initial products were not $\mathrm{TiO}_{2}$, but $\mathrm{Ti}(\mathrm{OH})_{4}$. Therefore, the TNT surfaces were hydrophilic as the reaction products contained hydroxide compounds.

The third phenomenon was that the annealing made the TNT surfaces superhydrophilic. In general, annealing metal generates a hydrophobic surface. ${ }^{112}$ 
Conversely, this study produced super-hydrophilic surfaces after annealing. The water droplet contact angles on the annealed TNT surfaces decreased 40 56\% compared to the as-anodized TNT surface cases. The annealing resulted in nanotubes of anatase causing super-hydrophilic surfaces to be generated. The other possibility is that the surface had become very rough. It might be possible that clusters of nanotubes on the annealed samples had peeled off revealing a rough underlying surface. This would make the water droplet spread over the surface. These areas were carefully avoided during the experiments.

The effect of aging of the TNT surfaces on wettability was examined during and after three months. Figure 7.6 shows representative images for the bare titanium, asanodized TNT, and annealed TNT. For the TNT surfaces, it was found that the surface wettabilities changed from hydrophilic to hydrophobic or from super-hydrophilic to hydrophilic after ageing. All of the TNT surfaces had the same tendency and the resulting water droplet contact angles are shown in Table 7.1. The contact angle analyses were made 22, 51, and 92 days after fabrication. The increasing hydrophobicity was different for each case but all of the water droplet contact angles increased on the TNT structured surfaces. Figure 7.7 (a) shows the variation of contact angles for the cases of the annealed and as-anodized TNT surfaces. Invariably, the results show that the surface hydrophobicity was increased for all four cases. The reason for this phenomenon can be explained by oxidation and molecular replacement. As seen in (3), $\mathrm{Ti}(\mathrm{OH})_{4}$ was one of the products of the anodizing process and the surface had hydrophilic properties because it consisted of hydroxyl groups. During aging however, the TNT surfaces experienced oxidation as seen in (4) because the titanium-dioxide $\left(\mathrm{TiO}_{2}\right)$ is a more stable material than the $\operatorname{Ti}(\mathrm{OH})_{4}$.

$$
\mathrm{Ti}(\mathrm{OH})_{4} \longrightarrow \mathrm{TiO}_{2}+2 \mathrm{H}_{2} \mathrm{O} \uparrow
$$




\begin{tabular}{|c|c|c|c|c|}
\hline & 0 day & 22 day & 51 day & 92 day \\
\hline $\begin{array}{r}\text { Annealed TS } \\
\text { (Pt/ alloy) }\end{array}$ & & & & \\
\hline $\begin{array}{r}\text { As-grown TS } \\
\text { (Pt/ alloy) }\end{array}$ & & & & \\
\hline $\begin{array}{c}\text { Bare foil } \\
\text { (alloy) }\end{array}$ & & & & \\
\hline
\end{tabular}

Fig. 7.6. Comparison of the time-dependent change of TNT surface wettability. As time passed, the TNT surface hydrophobicity increased.

Table 7.1 Variation of contact angle with respect to time

\begin{tabular}{|c|c|c|c|c|c|c|}
\hline \multicolumn{3}{|c|}{ Contact angle $\left(^{\circ}\right)$} & \multirow{2}{*}{$\begin{array}{l}0 \text { day } \\
10.76\end{array}$} & \multirow{2}{*}{$\begin{array}{l}22 \text { day } \\
21.85\end{array}$} & \multirow{2}{*}{$\begin{array}{l}51 \text { day } \\
23.39\end{array}$} & \multirow{2}{*}{$\begin{array}{l}92 \text { day } \\
26.94\end{array}$} \\
\hline Annealed & \multirow{2}{*}{$\mathrm{Pt}$} & Pure & & & & \\
\hline TNT & & Alloy & 14.59 & 19.45 & 31.56 & 44.12 \\
\hline As-grown & \multirow{2}{*}{$\mathrm{Pt}$} & Pure & 24.62 & 28.25 & 34.05 & 42.47 \\
\hline TNT & & Alloy & 32.39 & 37.97 & 42.23 & 80.35 \\
\hline \multirow{2}{*}{ Bare } & & Pure & 82.52 & 74.63 & 78.66 & 81.24 \\
\hline & & Alloy & 67.07 & 54.84 & 73.48 & 56.80 \\
\hline
\end{tabular}




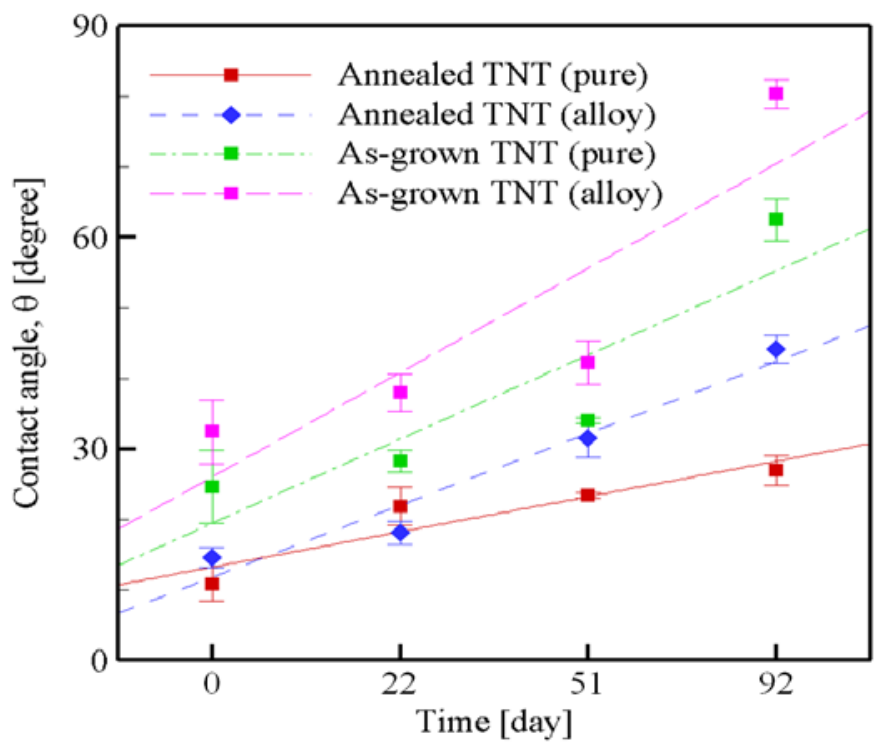

(a) Annealed and as-anodized TNT surfaces.

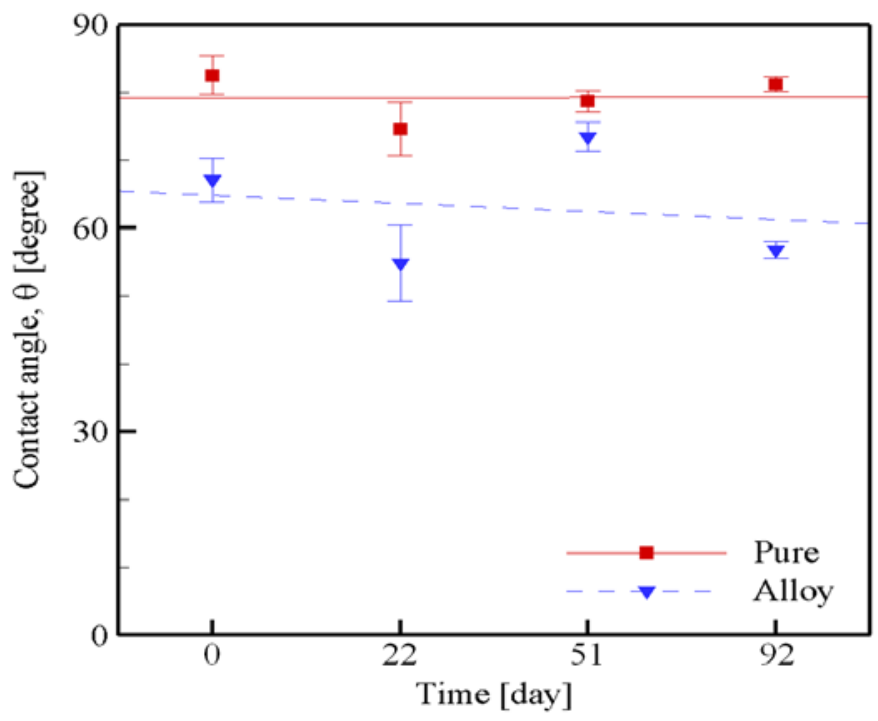

(b) The cases of bare titanium surfaces.

Figure 7.7. Comparison of the time-dependent contact angles, (a) shows the water droplet wettability on the annealed and as-anodized TNT surfaces. The increasing contact angles varied in each case but all of the TNT samples had the tendency of increasing the surface wettability. (b) shows the cases of bare titanium. In these cases, the wettability had little, if any, change much with time. 
$\mathrm{TiO}_{2}$ has hydrophobic characteristics and as time passed, the proportion of $\mathrm{TiO}_{2}$ increased due to aging of the TNT surfaces. The change in the wetting behavior of $\mathrm{TiO}_{2}$ porous structures would have significant impact for various applications. For example, there is often high sensitivity to different wetting characteristicss that affect the biological responses of protein adsorption, cell affinity or cell adhesion on a surface. Figure 7.7 (b) shows the wettability change for bare titanium and alloy as a function of ageing. The measured contact angles of droplets remained nearly unchanged for each case. At the early stage, the contact angles decreased a small amount due to the initial oxidation of the metal. Before the experiment, the initial titanium foils were cleaned with ethanol and acetone. These samples then became oxidized through exposure to the air. This caused an initial decrease of the contact angles. However, the wettabilities of the bare titanium foils remained constant with respect to time after this initial change.

Figure 7.8 shows the Fourier transform infrared (FTIR) spectroscopy results for the annealed and aged TNT, as-anodized and aged TNT, and as-anodized fresh TNT. FTIR spectroscopy was used to confirm the results and analysis of the prior observations. The hydroxide $\left(\mathrm{OH}^{-}\right)$stretching region is the section corresponding to wavenumbers between 3100 3400 $\mathrm{cm}^{-1}$. The FTIR analysis confirmed that the hydroxyl group was embedded in the fresh TNT surface (low transparence is high absorbance) and as the surface aged the hydroxide levels decreased (higher transparence). The annealed TNT had much lower hydroxyl group content than the as-anodized TNT surface. This means the substitution reaction speed would be accelerated around hydroxide and oxygen. This result also confirmed our theory that the $\mathrm{Ti}(\mathrm{OH})_{4}$ group was generated instead of $\mathrm{TiO}_{2}$ as a result of anodization. Then with the aging, the TNT structured surfaces changed from hydrophilic to hydrophobic. 


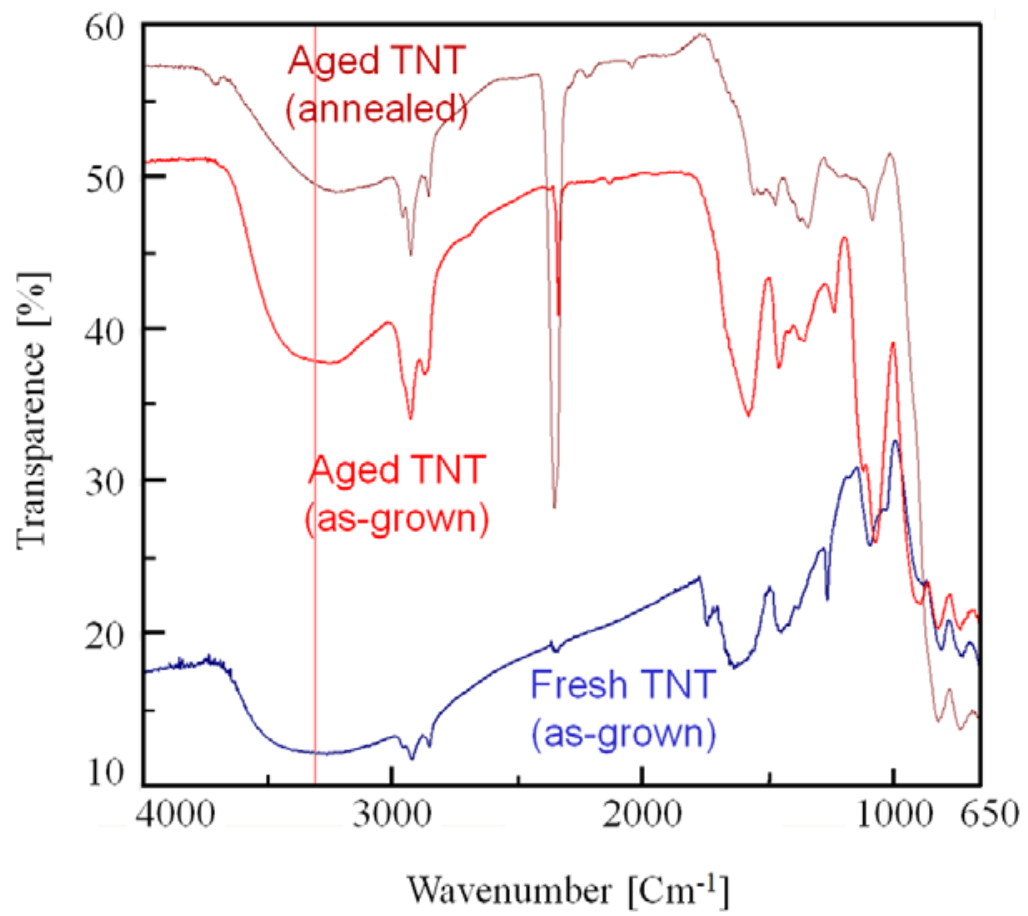

Figure 7.8. FTIR spectroscopy results for annealed and aged, as-anodized and aged, and as-anodized fresh TNT surfaces. It was confirmed that the fresh TNT surfaces had more hydroxyl groups on the surface than the aged TNT. The vertical line corresponds to approximately $3300 \mathrm{~cm}^{-1}$.

\subsection{Conclusions}

This study examined the wettability of bare titanium and alloy, and TNT structured and annealed and aged surfaces by analyzing the contact angles with $1 \mu \mathrm{de}$ ionized water droplets. From the gathered data, three conclusions were drawn. First, the TNT surfaces had a hydrophilic characteristic caused by the anodizing process and a surface initially with many hydroxyl groups instead of an oxide. As the chemical analysis indicated, and considering the degree of electrolytic dissociation, the surfaces were initially composed of $\mathrm{Ti}(\mathrm{OH})_{4}$ groups instead of $\mathrm{TiO}_{2}$ resulting in lower contact angles. Second, as a result of annealing the TNT surfaces became super-hydrophilic due to the generation of anatase. Extensive anodization can cause delamination of the TNT layer resulting in irregular surface roughness that can locally affect the contact angle. Third, 
aging of TNT surfaces leads to a marked the marked decrease of hydrophilicity. This is the result of the stabilization process for the substitution reaction from $\mathrm{Ti}(\mathrm{OH})_{4}$ to $\mathrm{TiO}_{2}$ as the hydroxyl groups disappear in air. This phenomenon was confirmed by FTIR spectroscopy. These results indicate that where surface wettability may be an advantage, nanotube growth by anodization using the process described here can satisfy this requirement. This may have extensive application for titanium orthopedic implants where the wettability characteristics of the surface can be specified.

\section{Acknowledgement}

This research was sponsored, in part, by a Michigan Technological University Research Initiation Grant (CKC) and by the Multi-Scale Technologies Institute (TS, CF). This work was also sponsored by the Manpower Development Program for Energy and Resources, supported by the Ministry of Knowledge and Economy (MKE) of the Korean government (SHL). 


\title{
Chapter 8
}

\section{$\mathrm{TiO}_{2}$ Nanotubes Interaction with Osteoblasts}

\begin{abstract}
After implantation of a biomaterial in the body, the interaction of the biomaterial with cells initially occurs on the surface of the implant. ${ }^{113}$ Therefore, understanding the surface properties of a biomaterial, such as roughness and wettability, are crucial for designing a successful implant for clinical application. However the mechanism in which these surface properties regulate cell function is not clearly understood. This chapter will mainly focus on the investigation of the effect of surface topography, crystallinity, composition and stiffness of $\mathrm{TiO}_{2}$ nanotubes on osteoblast growth. This chapter starts with a literature review on $\mathrm{TiO}_{2}$ nanotubes and their interactions with biological cells. I further extended the existing knowledge in the literature by investigating the effect of chemical composition, crystallography and stiffness of $\mathrm{TiO}_{2}$ nanotubes on osteoblast cell number and cell-nanotube interface qualification. The nanotubular characteristics of the surface improve cell number, attachment and spreading of osteoblast cells. The effect of chemical composition on osteoblast growth and adhesion by comparison between alloyed and pure $\mathrm{TiO}_{2}$ nanotubes was also investigated. Moreover I have investigated the osteoblast interaction and attachment with $\mathrm{TiO}_{2}$ nanotubes through focused ion beam cross sectional milling to reveal the osteoblast in depth growth into nanotubes.
\end{abstract}

\subsection{Literature Review}

The presence of nanoscale features on the surface of implants can in principal enhance the growth of osteoblast bone-forming cells. This is because nanometer features can mimic the natural environment that osteoblast are adapted to. For instance, osteoblasts have continuous interactions with hydroxypatite crystals that are $20-40 \mathrm{~nm}$ long and are located within a collagen matrix (Type I collagen is a triple helix $300 \mathrm{~nm}$ in length, $0.5 \mathrm{~nm}$ in width, and periodicity of $67 \mathrm{~nm}) .{ }^{114}$ This commonly used ceramic coating on metal implants has several issues due to the lack of mechanical properties such 
as cracking and delamination from the metal substrate. Therefore, development of novel coatings with more robust and flexible structures containing nanofeatures will be highly promising for better osseointegration, cell-implant interaction and implant life cycle. Self-assembled $\mathrm{TiO}_{2}$ nanotubes that are coated over the surface of Ti implants offer this flexibility and can mimic the natural environment of bone-forming cells.

Bjursten et al. ${ }^{115}$ showed that $\mathrm{TiO}_{2}$ nanotube surfaces have nine-fold higher boneimplant interlock in comparison to the sandblasted $\mathrm{TiO}_{2}$ surfaces. Their histological evaluation showed enhanced bone-implant contact area and increased calciumphosphorous levels on $\mathrm{TiO}_{2}$ nanotube surfaces. Hazan et al. ${ }^{116}$ evaluated the effect of surface topography of titania on fibroblast behavior. They compared three different surface structures including nanotubes, thin films, and foams of $\mathrm{TiO}_{2}$. Among all these three surfaces, $\mathrm{TiO}_{2}$ nanotubes showed the best properties for cell number.

Bauer et al. ${ }^{117}$ investigated $\mathrm{TiO}_{2}$ nanotube hydrophobicity on mesenchymal stem cells (MSCs) attachment. They used a monolayer coating to change the surface properties of nanotubes from hydrophilic to super-hydrophobic. They showed that hydrophobicity results in a higher MSC attachment but this behavior only lasted for a very short time. In a study by Yao et al. ${ }^{118}$ the effect of $\mathrm{TiO}_{2}$ nanotubes on human osteoblast cell function was investigated. They compared three different surface structures (smooth, nanoparticulate, and nanotubular) on osteoblast cell function. The $\mathrm{Ca}$ deposition by osteoblasts was highest on surfaces with $\mathrm{TiO}_{2}$ nanotubular features. They explained this finding based on the fact that nanotubes possess the highest surface area and surface energy among the other surface topographies. As such, nanotubes provide more reactive sites for fibronectin protein adsorbtion that mediates osteoblast adhesion.

Reports on the effect of nanotube diameter to the cell growth and adhesion are often contradictory. Park et al. ${ }^{119}$ studied the effect of titanium nanotube diameter on mesenchymal stem cell fate. They reported the optimized nanotube diameter to be $15 \mathrm{~nm}$. The cell attachment and adhesion increased in nanotubes of less that $50 \mathrm{~nm}$ diameter and decreased in nanotubes with diameter of more than $50 \mathrm{~nm}$. However, their finding was in contradiction with the results of Oh et al. ${ }^{120}$ and Brammer et al. ${ }^{121}$ who reported that the $100 \mathrm{~nm}$ nanotube size provides the highest MC3T3-E1 mouse osteoblast cell 
attachment and adhesion. Oh et al. ${ }^{122}$ also reported that the $100 \mathrm{~nm}$ tube size resulted in extremely elongated human MSCs force guiding them to differentiate into specific osteoblast cells.

Popat et al. ${ }^{123}$ investigated $\mathrm{TiO}_{2}$ nanotubular surfaces for better osseointegration and accelerated wound healing. Their experiments with marrow stromal cells indicated that in comparison to flat titanium surfaces, nanotubular $\mathrm{TiO}_{2}$ surfaces provided higher adhesion, alkaline phosphatase activity, and extracellular matrix production as well as enhanced calcium and phosphorus deposition resulting in upregulated bone matrix formation. In addition they investigated the biocompatibility of $\mathrm{TiO}_{2}$ nanotubes by implanting disks with nanotubular titania surfaces on rats. Their results showed no inflammatory response or fibrous tissue formation in the tissues surrounding the $\mathrm{Ti}$ implant due to the presence of $\mathrm{TiO}_{2}$ nanotubes.

\subsection{Experimental Procedure}

\subsubsection{Osteoblast Cell Culture Experiments}

For these studies, MC3T3-E1 mouse osteoblasts (CRL-2593, subclone 4, ATCC, USA) were used. ${ }^{124}$ Each $1 \mathrm{ml}$ of cells was mixed with $10 \mathrm{ml}$ of alpha minimum essential medium (a-MEM; Invitrogen, USA) with 10 vol.\% fetal bovine serum (FBS; Invitrogen, USA) and 1 vol.\% penicillin-streptomycin (PS; Invitrogen, USA). The cell suspension was placed in a cell culture dish and incubated at $37^{\circ} \mathrm{C}$ and 5 vol. $\% \mathrm{CO}_{2}$ environment. The concentration of the MC3T3-E1 osteoblastic cells were checked under an optical microscope every $24 \mathrm{hrs}$ until it reached its saturation point of approximately 3000 cells $/ \mathrm{mm}^{2}$ after $72 \mathrm{hrs}$ of incubation. When the cells reached their saturation point they were ready to be subcultured on $\mathrm{TiO}_{2}$ nanotube substrates. After that the MC3T3-E1 cells were seeded onto the experimental substrate of interest $\left(\mathrm{TiO}_{2}\right.$ nanotubes), they were placed on a 30 -well polystyrene plate and stored in $37{ }^{\circ} \mathrm{C} \mathrm{CO}_{2}$ incubator for 24,48 and $72 \mathrm{hrs}$ to observe the cell morphology and count the number of viable cells attached as a function of incubation time. 


\subsubsection{Osteoblast Cell Viability Determination}

Determination of cell viability depends on if the cell is able to actively adhere to the substrate. An active cell looks healthy and is able to proliferate after attachment to the surface. Considering this fact, these experiments were conducted as a pre-evaluation of cell viability of osteoblasts cultured on $\mathrm{TiO}_{2}$ nanotubes and to see if the cells are able to attach, spread, and grow on these substrates. Osteoblast cells were cultured on 6-well single plate culture dishes and evaluated for viability after $1 / 2 \mathrm{hr}, 2 \mathrm{hrs}$ and $48 \mathrm{hrs}$ of culture. A plate containing only culture media was used as the control. Optical microscopy was used for imaging the cultured cells. The results are presented in section 8.3 and figure 8.1. After $1 / 2 \mathrm{hr}$ of culture the cells were round showing no attachment to the surface in all samples including the control sample. After $2 \mathrm{hrs}$ of culture the cells started to change their shape to a more spread out appearance and the number of cells increased. After $48 \mathrm{hrs}$ of culture, the number of cells further increased and their shape became flattened because of spreading and attachment. Since the osteoblasts were keeping the healthy shape and were able to proliferate, they appeared healthy and active. These results showed the viability of osteoblast cells on $\mathrm{TiO}_{2}$ nanotube surfaces and were in agreement with literature studies on cytotoxcicity evaluations of $\mathrm{TiO}_{2}$ nanotubes confirming the non-toxic nature of the nanotubes ${ }^{125,126,127,128}$.

\subsubsection{Cell Number, Attachment and Spreading on Different Types of $\mathrm{TiO}_{2}$ Nanotube Substrates}

These experiments were conducted to evaluate the effect of $\mathrm{TiO}_{2}$ nanotube topography, wettability, crystallinity, and chemical composition on osteoblast cell number (see section 8.4), attachment and spreading (see section 8.6). The effect of $\mathrm{TiO}_{2}$ nanotube stiffness on osteoblast cell number is presented in section 8.7.

The common way of determining cell number is to first detach all incubated cells from the substrate surface with Trypsin/EDTA. The suspension with detached cells is then collected into a test tube. The detaching process will be repeated until no residual cells are found on the substrate surfaces after several Trypsin treatments. Released 
cells will be counted with a hemocytometer. This method of cell count has some limitations. The main limitation is that all the cells, including the cells in the culture media around the substrates are suspended as well. Since these cells were not cultured on the same substrate they do not represent the actual condition but instead a mixture with the control condition. Therefore the mixture of these cells in the suspension is not an accurate evaluation of the cell count on the specific titanium substrate. Another limitation is that during the cell count by hemocytometer all the cells will have an equal spherical shape because they are all in suspension. But only the flat and well-spread cells are indications of viable cells on the titanium surface. In other words the common method of cell counting is not accurate enough to distinguish between cells of the control condition including the culture dish and media surrounding the sample, non-attached round cells, and well-spread, attached cells. Therefore a different method of cell counting was used. By rapid fixation of the cells and immediate SEM imaging of the substrate, only the wellspread attached cells were counted toward the cell number investigation. The highresolution imaging gave the capability of distinguishing between non-flat and well-spread flat cells and the rapid fixation got rid of the cells in the control condition, resulting in a more accurate number assay.

Substrates of $\mathrm{TiO}_{2}$ nanotubes anodized at $60 \mathrm{~V}$ were used for cell number experiments. Prior to cell culture, substrates were ultrasonically cleaned and sterilized in $70 \%$ ethanol for $15 \mathrm{~min}$, washed in deionized water, and air dried under a laminar flow hood. Each group of substrates used for cell number tests was divided into the three following subgroups. Substrates of as-anodized titanium (4 substrates of commercially pure (cp)- $-\mathrm{TiO}_{2}$ nanotubes and 4 substrates of alloyed $\mathrm{TiO}_{2}$ nanotubes), annealed nanotubes (4 substrates of $\mathrm{cp}-\mathrm{TiO}_{2}$ nanotubes and 4 substrates of alloyed $\mathrm{TiO}_{2}$ nanotubes), and control bare titanium (4 substrates of cp-Ti and 4 substrates of Ti alloy (Ti6A14V)) were put into 24-well plates with one sample located in each well. MC3T3 cells with a density of approximately 125 cells $/ \mathrm{mm}^{2}$, suspended in $5 \mathrm{ml}$ cell culture medium, were seeded onto the surfaces of substrates, and incubated with humidified atmosphere with $5 \% \mathrm{CO} 2$ at $37^{\circ} \mathrm{C}$. After $24 \mathrm{hr}, 48 \mathrm{hrs}$ and $72 \mathrm{hrs}$, respectively, the samples were rinsed with PBS and immediately fixed on the titanium samples 
for SEM evaluation. For cell fixation, 90\% ethanol was added to the samples. After 10 min the ethanol was pipetted out and the samples were left to air dry at $37{ }^{\circ} \mathrm{C}$ for $15 \mathrm{~min}$. The results are presented in Section 8.4.

\subsubsection{FIB Milling Procedure}

For the purpose of the FIB and SEM investigations, the cells were fixed on the nanotube substrates. After $72 \mathrm{hrs}$ of culture the medium was removed from each culture plate. After that $90 \%$ ethanol was added to the substrate. After 10 min the ethanol was pipetted out and the substrates were left to air dry at $37^{\circ} \mathrm{C}$ for $15 \mathrm{~min}$. After cell fixation the substartes were used for the SEM and FIB experiments. Substrates with fixed cells were inserted into the specimen chamber of a FIB (Hitachi SA-2000). The rough milling conditions to open a trench in the cell used ion currents of 5 to $7 \mathrm{nA}$ at $30 \mathrm{kV}$. Lower beam currents of 100 to $670 \mathrm{pA}$ were used to polish the cross section. Dwell time for milling was $3 \mu$ s. Results are reported in section 8.6.

\subsection{Effect of Nanotubes on Cell Attachment and Spreading}

Cell spreading was assessed by optical microscopy examination of all substrates prepared during cell culture experiments described in section 8.2.2. It should be noted that the common way of investigating cell attachment and spreading is by vinculin staining to show focal contacts and actin filaments probing with FITC-conjugated phalloidin to reveal the cytoskeleton arrangement. ${ }^{129}$ Since this method is not time/cost effective, a different method of investigation was performed using optical microscopy and SEM imaging. More importantly, using this method of high resolution imaging at the nano-level, a more in-depth evaluation of cell appearance and cell filopodia expansion was possible (see Section 8.6).

Figure 8.1 shows cell viability determination by optical microscopic imaging and observation of cultured cells on the control culture dish and $\mathrm{TiO}_{2}$ nanotubes. The cell viability was examined by evaluation of cell activity after $1 / 2 \mathrm{hr}, 2 \mathrm{hrs}$, and $48 \mathrm{hrs}$ of culture. The cell number, attachment and spreading can be observed, which are the 
indication of cell viability. The morphology of the cells can be categorized into (A) no cell spreading, cells were still spherical in appearance, no protrusions or lamellipodia were produced; (B) partially spread cells, at this stage, cells began to spread laterally, at one side or more, but the extensions of plasma membrane were not completely confluent; and (C) fully spread cells, sufficient extension of the plasma membrane or flattening of the cells was obviously observed.

As discussed, the morphological changes of cell shape during the process of cellsubstrate attachment can be divided into three steps; early stage with no spreading; partially spread; and well-spread shape. A viable cell is able to undergo all these three steps prior to the complete surface attachment. Since the osteoblasts kept the morphology of a viable cell and were able to proliferate, they appeared healthy and active. These results show the viability of osteoblast cells on $\mathrm{TiO}_{2}$ nanotube surfaces and are in agreement with literature studies on cytotoxcicity evaluations of $\mathrm{TiO}_{2}$ nanotubes confirming the non-toxic nature of the nanotubes. ${ }^{130,131,132,133}$

(A) $1 / 2 \mathrm{hrs}$ (B) $2 \mathrm{hrs}$ (C) $48 \mathrm{hrs}$

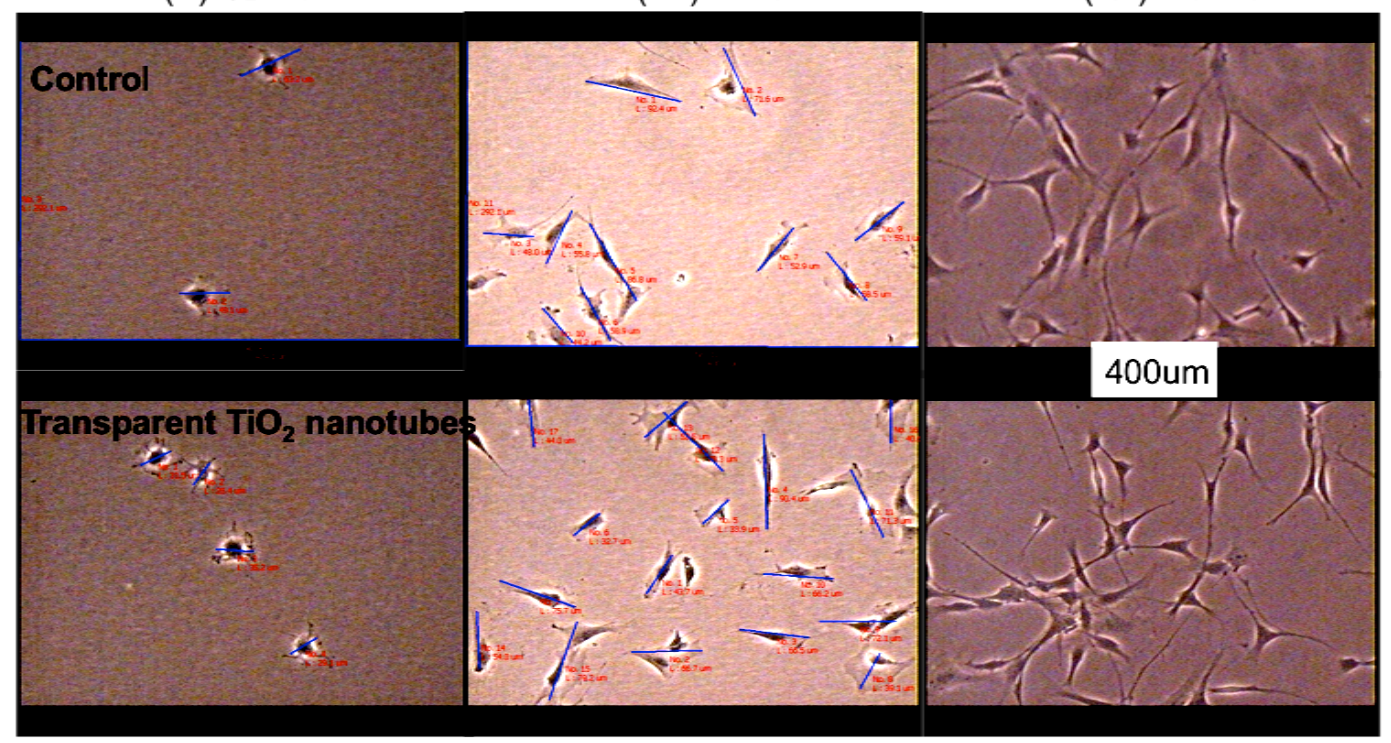

Figure 8.1. Cell viability determination by microscopic imaging and observation of cell activity such as number, attachment as spreading after (A): no cell spreading; $1 / 2 \mathrm{hr}$, (B):

partially spread cells; 2 hrs, (C) fully spread cells; 48 hrs. The field of view is $400 \times 300 \mu \mathrm{m}^{2}$. 


\subsection{Effect of Nanotubes Topography, Wettability, Crystallinity, and Chemical Composition on Osteoblast Cell Number}

After evaluating the cell viability and adhesion on $\mathrm{TiO}_{2}$ nanotubes, the cell count experiments were conducted. As discussed earlier by rapid fixation of the cells and immediate SEM imaging of the substrate, only the well-spread attached cells were counted toward the cell number investigation. The high-resolution imaging gave the capability of distinguishing between non-flat and well-spread flat cells and the rapid fixation got rid of the cells in the control condition, resulting in a more accurate cell count assay. The mean value numbers of total attached cells were calculated from total cell number counted from 5 different random square areas $\left(1.0 \times 1.0 \mathrm{~mm}^{2}\right)$ of each substrates. The mean value number of attached cells on the surfaces of the control and the data of the other samples were calculated and compared for 24, 48 and $72 \mathrm{hrs}$ of incubation.

Figure 8.2 shows representative images of initial water droplets on bare Ti foil, pure $\mathrm{TiO}_{2}$ nanotubes, and alloyed $\mathrm{TiO}_{2}$ nanotubes in as-anodized and annealed conditions. Three phenomena were observed. The first was the difference between the contact angles of the bare pure and bare alloy titanium foils (Figure 8.2.c and f). In the case of pure titanium, the contact angles were larger than the alloy. Contact angle differences were attributed to the surface properties, in particular the surface roughness, which could affect the initial contact angle of a water droplet on each titanium surface. Second, it was found that the creation of $\mathrm{TiO}_{2}$ nanotube structures made the surface more hydrophilic (Figure 8.2.b and e). For all cases after the anodization process, the droplet contact angles decreased in the range of 55 70\% compared to the contact angles of each bare titanium foil. Third, the water droplet contact angles on the annealed $\mathrm{TiO}_{2}$ nanotube surfaces decreased $40 \sim 56 \%$ compared to the as-anodized $\mathrm{TiO}_{2}$ nanotube surface cases (Figure 8.2.a and $\mathrm{d}$ ). The annealing resulted in nanotubes of anatase causing superhydrophilic surfaces to be generated. According to Figure 8.2 it was shown that the hydrophilicity of anatase nanotubes is the highest among the amorphous $\mathrm{TiO}_{2}$ 
nanotubes and bare-Ti surfaces resulting in higher cell growth on such surfaces (figure 8.4 and 8.6). These results are in agreement with $\mathrm{Oh}$ et al. ${ }^{134}$ who investigated the effect of titanium nanotubes with different crystallographic structures on MC3T3-E1 osteoblast cells. They concluded that in comparison to conventional smooth titanium surfaces, nanotubes improve the speed of cell growth up to $400 \%$. Their results showed that the speed of cell growth was the highest in anatase $\mathrm{TiO}_{2}$ nanotubes in comparison to asanodized amorphous nanotubes. The authors have not discussed clearly why the crystallographic change has lead to the increase in cell growth.
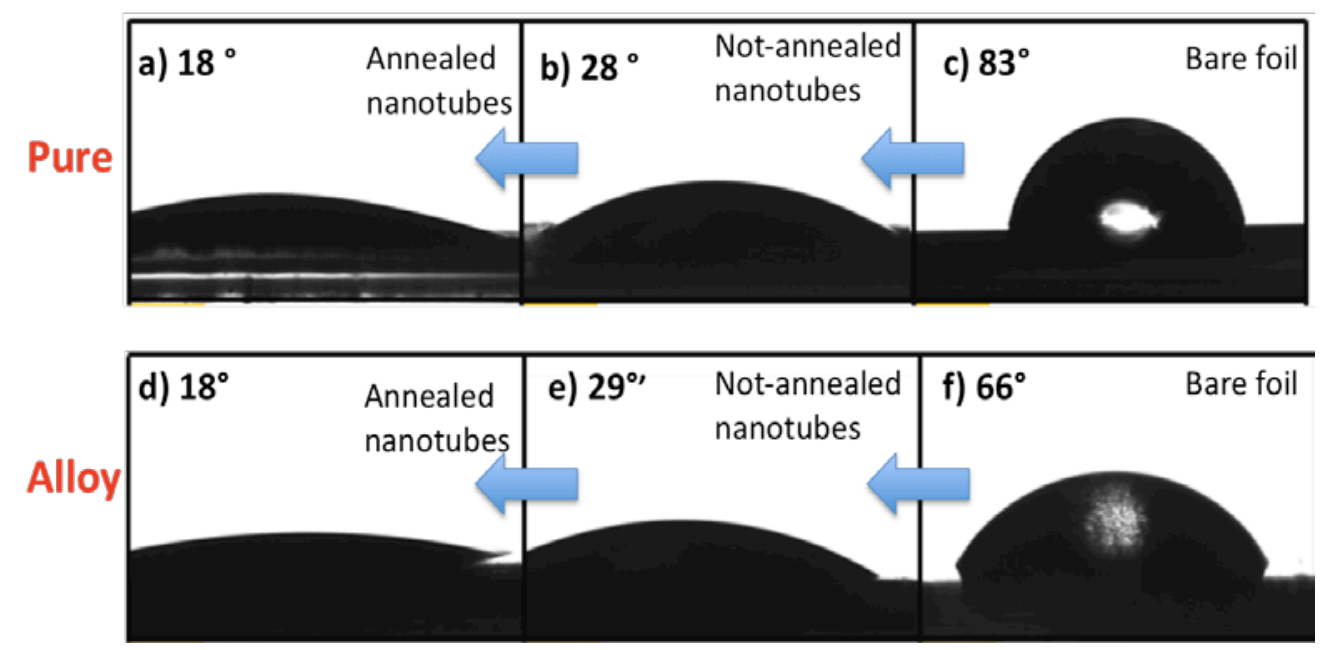

Figure 8.2. (a-c) Contact angle measurements for the $\mathrm{cp}-\mathrm{TiO}_{2}$ nanotube surface, (a) annealed cp-Ti surface, (b) not annealed cp-Ti surface, (c) bare cp-titanium surface. (d-f) (d) Contact angle measurements for the Ti6AL4V nanotube surface, annealed Ti6A14V,

(f) not annealed Ti6A14V nanotube surface (e), bare Ti6A14V surface.

Figure 8.3 shows osteoblast cell culture experiments on control cp-Ti and amorphous cp- $\mathrm{TiO}_{2}$ nanotubes as a function of incubation time. The number of cells was estimated from FE-SEM images of the substrates by an image processing threshold of $30 \%$ contrast adjustment between cells and $\mathrm{TiO}_{2}$ nanotube substrates and a cell count tolerance of \pm 5 cells $/ \mathrm{mm}^{2}$. As seen in Figure 8.3a-c, on the control cp-Ti substrate, cell count increased from 40 cells $/ \mathrm{mm}^{2}$ after $24 \mathrm{hrs}$ (Figure $8.3 \mathrm{a}$ ) to 300 cells $/ \mathrm{mm}^{2}$ after 72 
hrs of incubation (Figure 8.3d). The results indicated that the presence of nanotube morphology enhanced cell number from $80 \mathrm{cells} / \mathrm{mm}^{2}$ after 24 hrs (Figure $8.3 \mathrm{~d}$ ) to 900 cells $/ \mathrm{mm}^{2}$ after $72 \mathrm{hrs}$ of incubation (Figure 8.3f). This enhanced cell number is in agreement with the work of $\mathrm{Oh}$ et al. discussed earlier and the literature, ${ }^{135}$ on the effect of substrate topography and roughness on inducing cell growth. However, as shown in Figure $8.2 \mathrm{~b}$ and $\mathrm{c}$, I believe that not only nanotopography but also improved surface hydrophilicity can result in enhanced cell growth. It has been confirmed by the literature that higher surface hydrophilicity improves cell number due to higher density of $\mathrm{OH}$ groups and higher surface energy resulting in enhanced protein adsorption. ${ }^{136}$ 


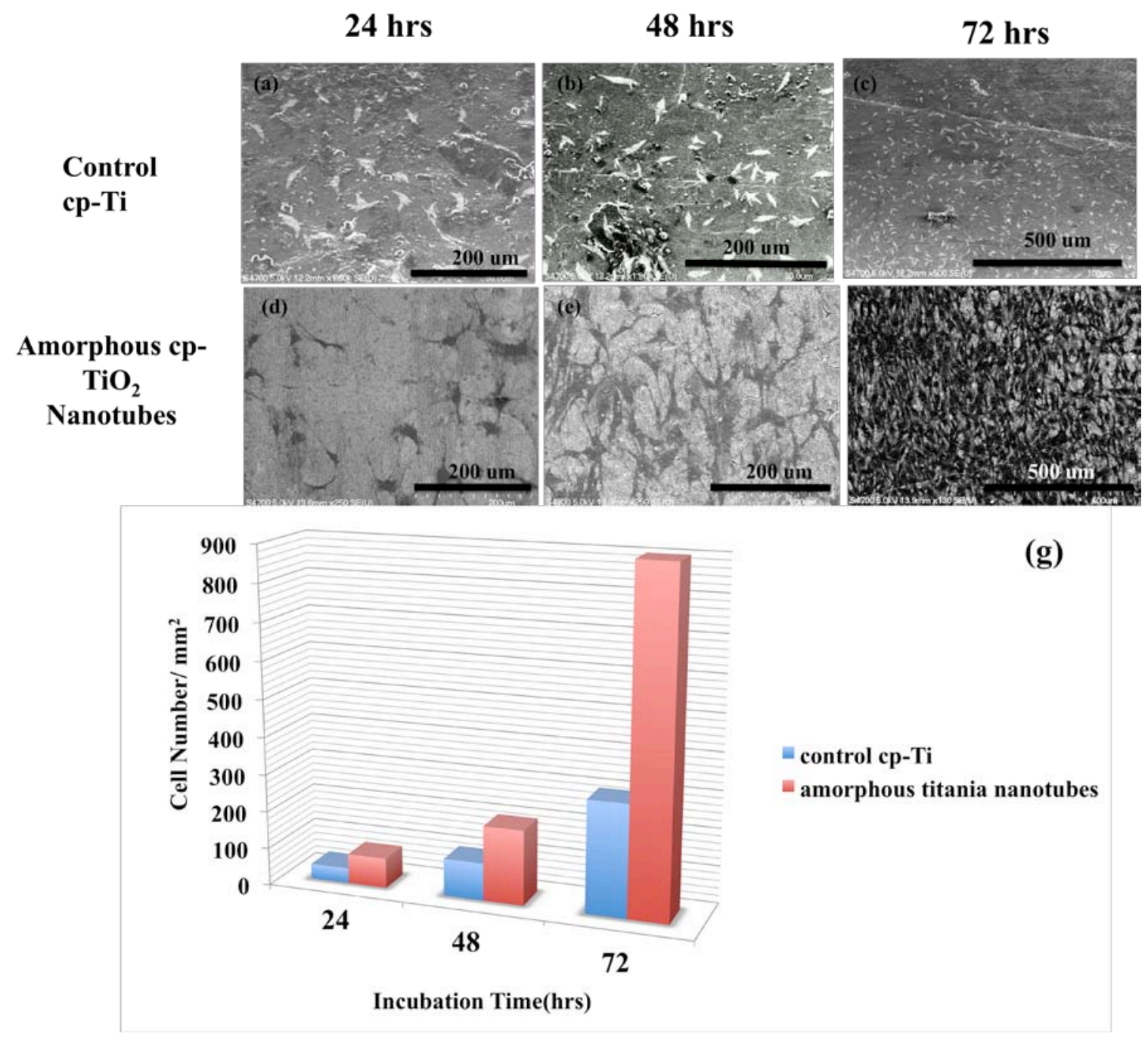

Figure 8.3. SEM micrograph of MC3T3 cells after $24 \mathrm{hrs}, 48 \mathrm{hrs}$ and $72 \mathrm{hrs}$ of culture on control cp-Ti (a-c) and as anodized amorphous cp- $\mathrm{TiO}_{2}$ nanotubes (d-f). Cell number increases as a function of culture time in both control and anodized samples. The number of attached cells increases by anodization (d-f). Scale bar (a, b, d, e) is $200 \mu \mathrm{m}$, and ( c, f) is $500 \mu \mathrm{m}$.

Figure 8.4 shows results of osteoblast cell culture experiments on annealed cp$\mathrm{TiO}_{2}$ nanotubes as a function of incubation time. As seen in Figure 8.4a-c, substrate cell count increased from 300 cells $/ \mathrm{mm}^{2}$ after 24 hrs (Figure 8.4a) to 2500 cells $/ \mathrm{mm}^{2}$ after 72 
hrs of incubation (Figure 8.4d). These results indicate that annealed nanotube morphology further enhanced cell number from 900 cells $/ \mathrm{mm}^{2}$ in amorphous (as-anodized) nanotubes (Figure $8.3 \mathrm{f}$ and d) to $2500 \mathrm{cells} / \mathrm{mm}^{2}$ in annealed nanotubes (Figure $8.4 \mathrm{c}$ and d) after 72 hrs of incubation. As discussed, I believe that not only nanotopography but also improved surface hydrophilicity resulted in further enhancement of cell number.

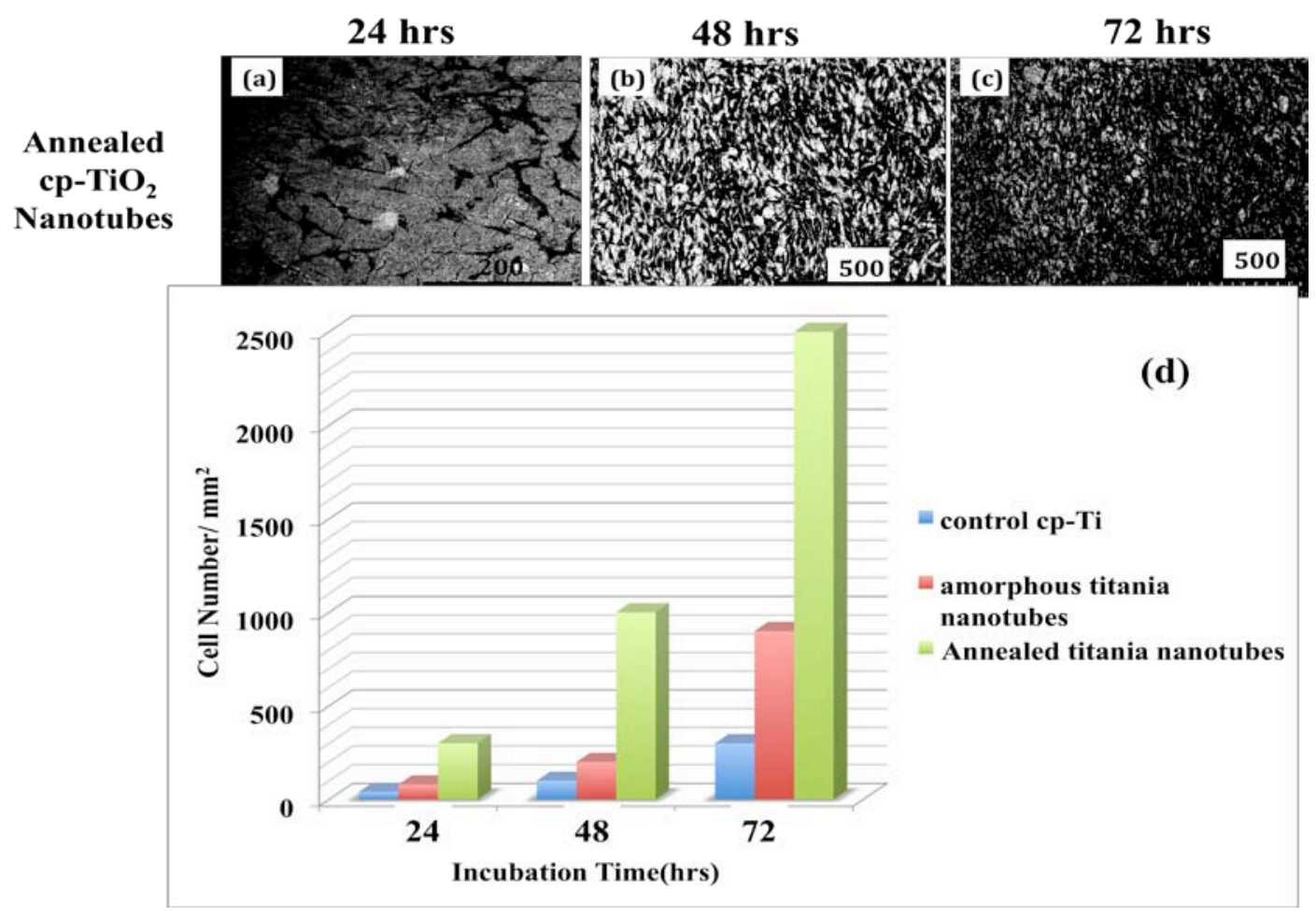

Figure 8.4. SEM micrograph of MC3T3 cells after $24 \mathrm{hrs,}, 48 \mathrm{hrs}$ and $72 \mathrm{hrs}$ of culture on annealed $\mathrm{cp}-\mathrm{TiO}_{2}$ nanotubes (a-c). Cell number increases as a function of culture time. The number of attached cells increases by annealing (d). Scale bar (a) is $200 \mu \mathrm{m},(b, c)$ is $500 \mu \mathrm{m}$.

Figure 8.5 shows the results of osteoblast cell culture experiments on the control Ti6Al4V and amorphous alloyed- $\mathrm{TiO}_{2}$ nanotubes as a function of incubation time. As seen in Figure 8.5a-c, on the control Ti6Al4V substrate cell count increased from 12 cells $/ \mathrm{mm}^{2}$ after $24 \mathrm{hrs}$ (Figure 8.5a) to 200 cells $/ \mathrm{mm}^{2}$ after $72 \mathrm{hrs}$ of incubation 
(Figure $8.5 \mathrm{~d}$ ). Our results indicate that nanotube morphology enhances cell number from 40 cells $/ \mathrm{mm}^{2}$ after $24 \mathrm{hrs}$ (Figure $8.5 \mathrm{~d}$ ) to 800 cells $/ \mathrm{mm}^{2}$ after $72 \mathrm{hrs}$ of incubation (Figure 8.5f). This enhanced cell number is in agreement with the results on cp-Ti and cp- $\mathrm{TiO}_{2}$ nanotubes shown in Figure 8.5. However, the total cell counts in the control Ti6Al4V and alloyed- $\mathrm{TiO}_{2}$ nanotubes is slightly lower for all incubation periods, in comparison to the total cell count in cp-Ti and cp- $\mathrm{TiO}_{2}$ nanotubes. This could be due to lower biocompatibility of the alloying elements ( $\mathrm{Al}$ and $\mathrm{V}$ ) and their oxides. ${ }^{137,138}$ 


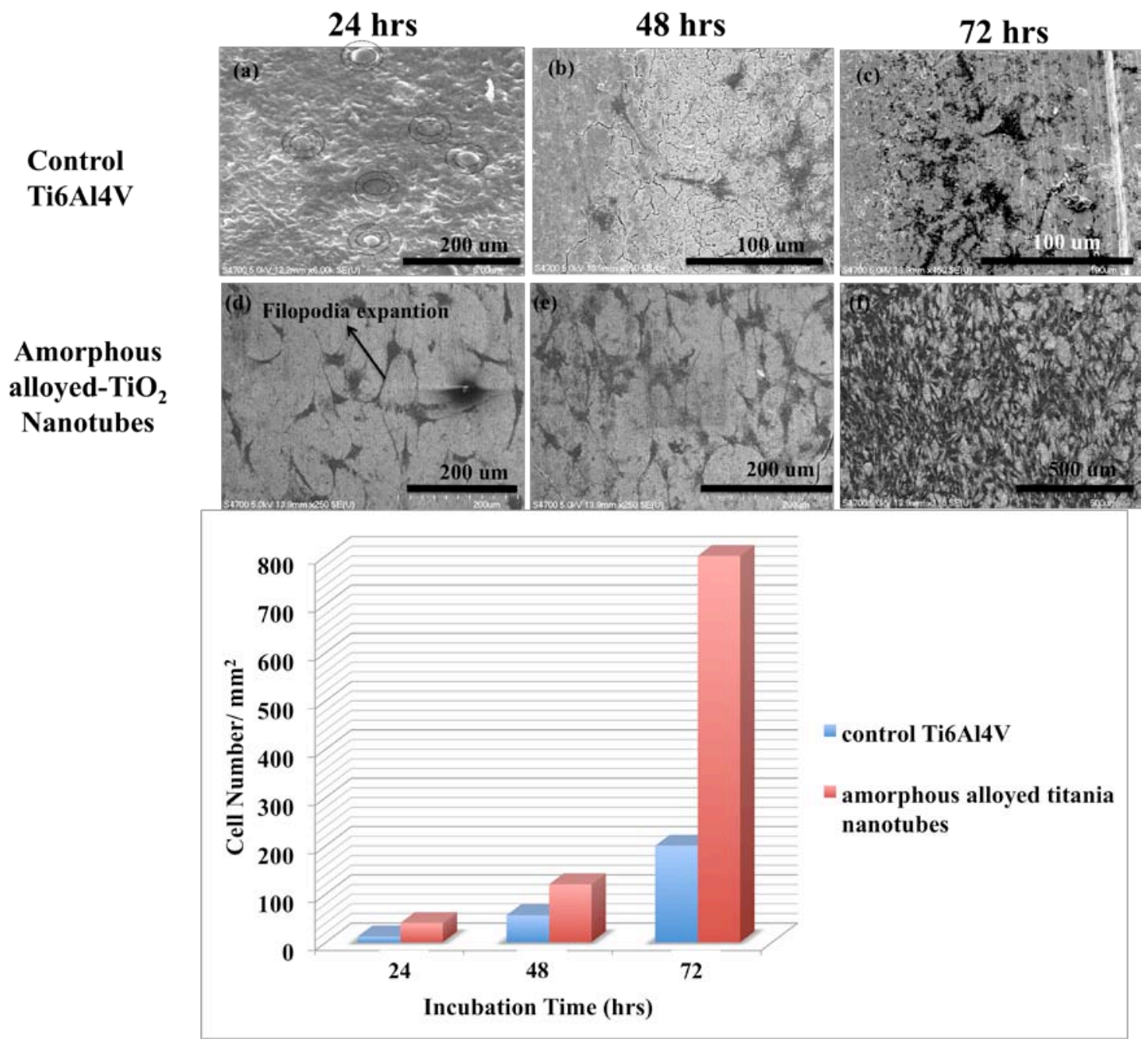

Figure 8.5. SEM micrograph of MC3T3 cells after $24 \mathrm{hrs}, 48 \mathrm{hrs}$ and $72 \mathrm{hrs}$ of culture on control Ti6Al4V (a-c) and as-anodized amorphous alloyed- $\mathrm{TiO}_{2}$ nanotubes (d-f). Cell number increased as a function of culture time in both control and anodized samples. The number of attached cells increased by anodization (d-g). Scale bar (a,d,e) is $200 \mu \mathrm{m},(\mathrm{b}, \mathrm{c})$ is $100 \mu \mathrm{m}$, and (f) is $500 \mu \mathrm{m}$.

Figure 8.6 shows results of osteoblast cell culture experiments on annealed alloyed $\mathrm{TiO}_{2}$ nanotubes as a function of incubation time. As seen in Figure 8.6a-c, substrate cell count increased from 200 cells $/ \mathrm{mm}^{2}$ after $24 \mathrm{hrs}$ (Figure 8.6a) to 2000 cells $/ \mathrm{mm}^{2}$ after $72 \mathrm{hrs}$ of incubation (Figure $8.6 \mathrm{~d}$ ). These results indicated that annealed nanotube morphology further enhanced cell number from 800 cells $/ \mathrm{mm}^{2}$ in amorphous 
(as-anodized) alloyed nanotubes (Figure $8.5 \mathrm{f}$ and $\mathrm{g}$ ) to $2000 \mathrm{cells} / \mathrm{mm}^{2}$ in annealed nanotubes (Figure 8.6c and d) after $72 \mathrm{hrs}$ of incubation. As discussed, not only nanotopography, but also improved surface hydrophilicity, has resulted in further enhancement of cell number. However, similar to the case for the control Ti6Al4V and alloyed- $\mathrm{TiO}_{2}$ nanotubes, the total cell counts in annealed alloyed $\mathrm{TiO}_{2}$ nanotubes is also slightly lower for all incubation periods, in comparison to the total cell count on annealed cp- $\mathrm{TiO}_{2}$ nanotubes. As discussed and referenced in section 8.5, this could be due to lower biocompatibility of the alloying elements ( $\mathrm{Al}$ and $\mathrm{V}$ ) and their oxides which have preserved their properties even after annealing.

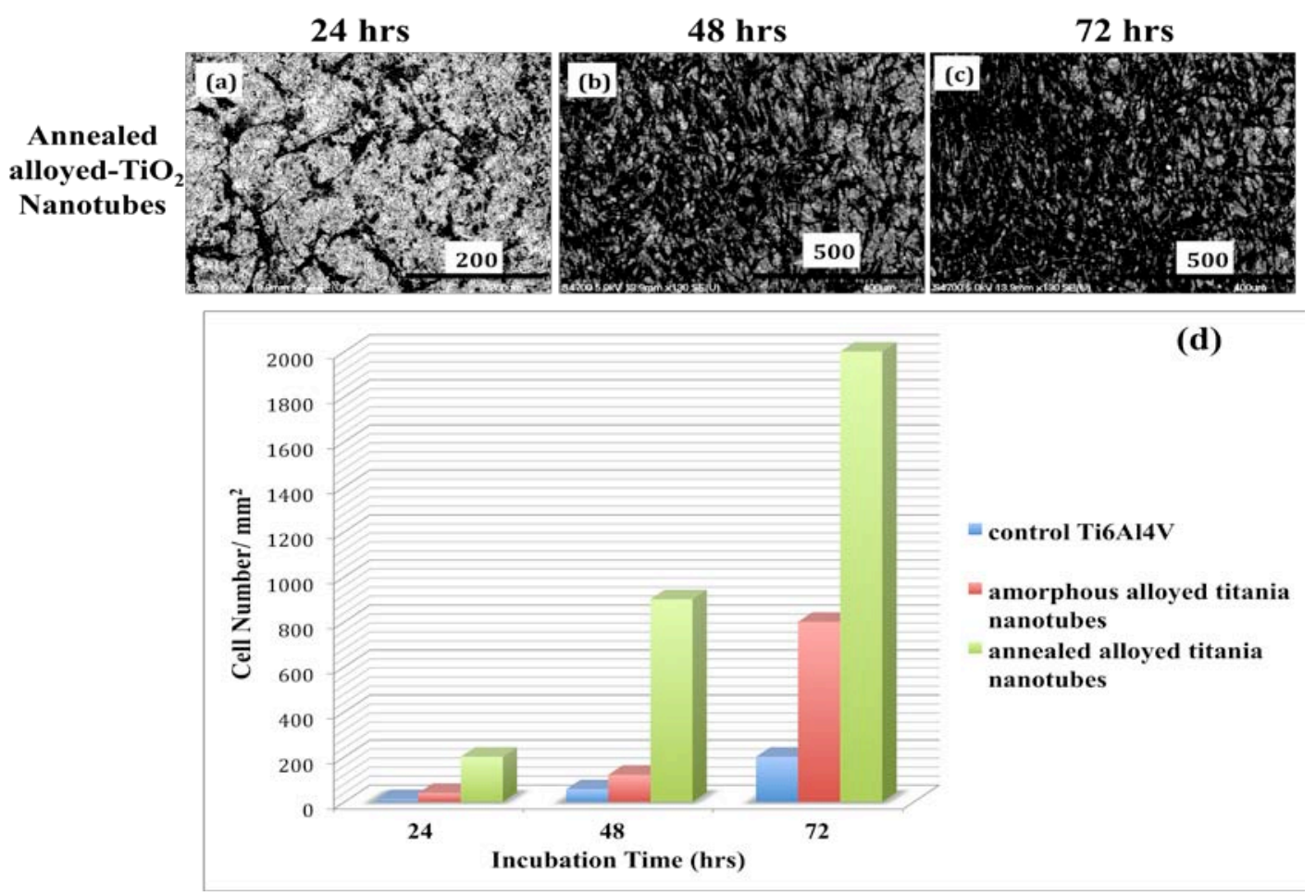

Figure 8.6. SEM micrograph of MC3T3 cells after $24 \mathrm{hrs,} 48 \mathrm{hrs}$ and $72 \mathrm{hrs}$ of culture on annealed alloyed $\mathrm{TiO}_{2}$ nanotubes (a-c). Scale bare (a) is $200 \mu \mathrm{m}$ and (b,d) is $500 \mu \mathrm{m}$.Cell number increases as a function of culture time. The number of attached cells increases by annealing (d). Scale bar (a) is $200 \mu \mathrm{m}$, and (b,c) is $500 \mu \mathrm{m}$.

Figure 8.7 was obtained from comparisons and combining the graphs of Figure $8.4 \mathrm{~d}$ and $8.6 \mathrm{~d}$. This figure shows the effect of anodization and annealing in enhancing 
cell number and growth in comparison to control bare surfaces for both $\mathrm{cp}$ and alloyed substrates. In the cell count of annealed samples there is a slight increase in the case of $\mathrm{cp}$ nanotubes compared to alloyed nanotubes that could be due to higher biocompatibility of cp Ti.

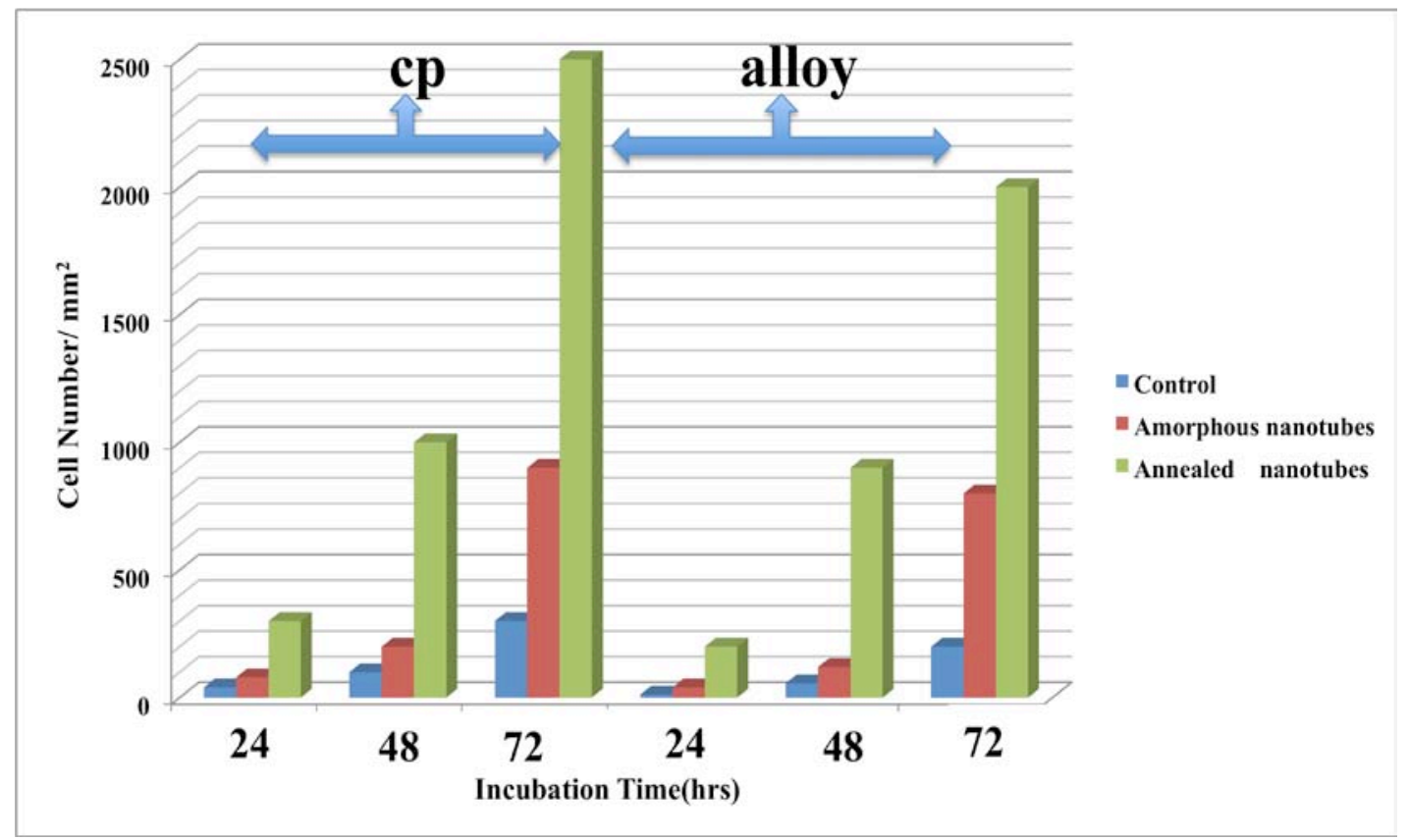

Figure 8.7. Number of adhered cells as a function of incubation time on the surface of bare $\mathrm{Ti}$ (left) and $\mathrm{TiO}_{2}$ nanotubes (right). The data of this chart has been obtained from comparison and combining the graphs of Figures $8.4 \mathrm{~d}$ and $8.6 \mathrm{~d}$. The data confirms the effect of anodization and annealing in enhancing number and growth in comparison to control bare surfaces for both cp and alloyed samples. However, in the cell count of annealed samples there is a slight increase in the case of cp nanotubes compared to alloyed nanotubes which could be due to higher biocompatibility of cp Ti.

Figure 8.8. shows the number of MC3T3 cells proliferated after $72 \mathrm{hrs}$ of incubation on $\mathrm{TiO}_{2}$ nanotubes with varying crystallinity and anodization duration. As discussed earlier, annealed nanotubes posses a crystalline structure with super hydrophilic properties, which results in higher cell number (2000 cells/ mm) in 
comparison with amorphous nanotubes $\left(800\right.$ cells/ $\left.\mathrm{mm}^{2}\right)$. Moreover varying the anodization duration, the length and diameter of the nanotubes change and results in longer and larger nanotubes. This change in nanotube geometry apparently affects the osteoblast cell number. Also, as discussed earlier, the literature shows that nanotube diameter affects cell number. However there is lack of data on the effect of nanotube length and therefore structural stiffness on cell growth. Here in, I show that not only diameter and nanotube length affects cell number. The results indicated that nanotubes with the same crystalline structure and chemical composition, but higher anodization duration and consequently higher length and diameter, showed a decrease in the number of attached cells. As seen in Figure 8.8 in the case of amorphous (as anodized) $\mathrm{TiO}_{2}$ nanotubes, the anodization duration of 240 min shows a higher number of attached cells $\left(2000\right.$ cells $\left./ \mathrm{mm}^{2}\right)$ in comparison to nanotubes anodized for $960 \mathrm{~min}\left(800\right.$ cells $\left./ \mathrm{mm}^{2}\right)$. 

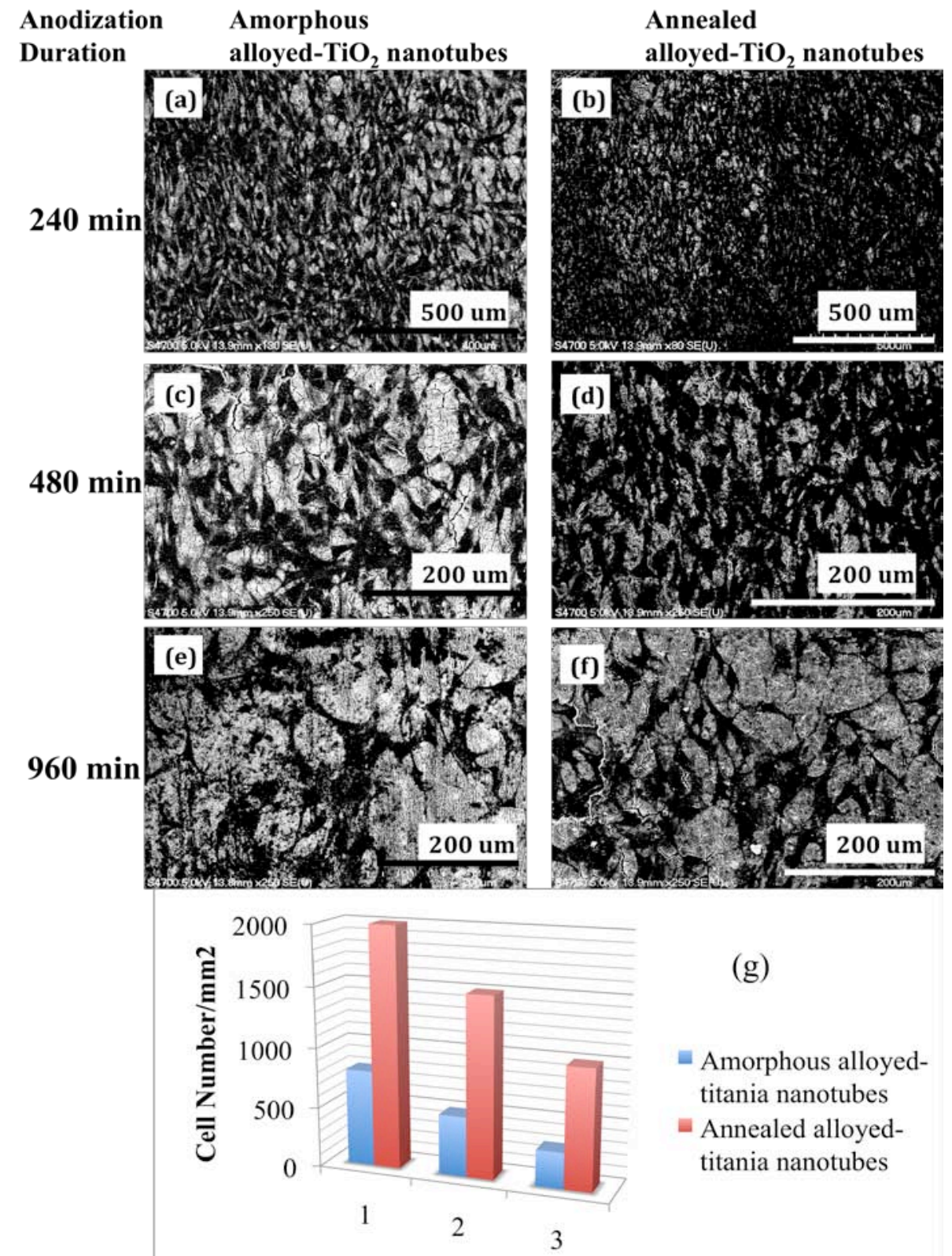

Anodization Duration (min)

Figure 8.8. The effect of nanotube length, crystallinity and composition on cell number and attachment. Scale bar (a,b) is $500 \mu \mathrm{m}$, and (c,d,e,f) is $200 \mu \mathrm{m}$.

This finding signifies the importance of considering both the diameter and length of nanotubes on cell number and attachment. The main reason for the nanotube geometric effect (diameter and length) on osteoblast cell number and attachment is due to a change 
in nanotube stiffness, which will be discussed in Section 8.7. Longer nanotubes are more flexible compared to shorter nanotubes. Since the substrate stiffness plays an important role in cell number, attachment and motility, varying the nanotube stiffness may result in changes in cell behavior. In Section 8.7, it is shown that shorter nanotubes have higher stiffness and therefore is easier for the cells to grab onto them, using them as anchoring steps for their attachment and movement. Cells use their filopodia as legs to move on surfaces. It is easier to walk on stiffer substrates than flexible substrates because it works better as an anchor or step. On stiff substrates, resistance to mechanical probing may lead to protein conformational changes and activation of signaling enzymes at the adhesion sites ${ }^{139}$. This phenomenon can be explained by the mechanism of force probing by cells.

\subsection{Preferred Directionality of Osteoblast Cell Growth}

Figure 8.9 shows SEM micrographs of osteoblast cells when cultured on a control $\mathrm{Ti}$ substrate (Figure 8.9.a, without nanotubes), and on $\mathrm{TiO}_{2}$ nanotubes. The $\mathrm{TiO}_{2}$ nanotubes may have affected the alignment of the cells parallel to the nanotopography direction, which is in agreement with the literature. Independent of mechanical loading, the morphology of the substrate has been reported to affect bone cell mechanobiology in a similar way that mechanical loading does. Microscale grooves have been shown to

cause contact guidance and alignment parallel to the groove direction. ${ }^{140}$ This effect of substrate topographical structures on cell alignment is important since it has been shown to affect gene expression and differentiation capacity. ${ }^{141,142,143}$ 

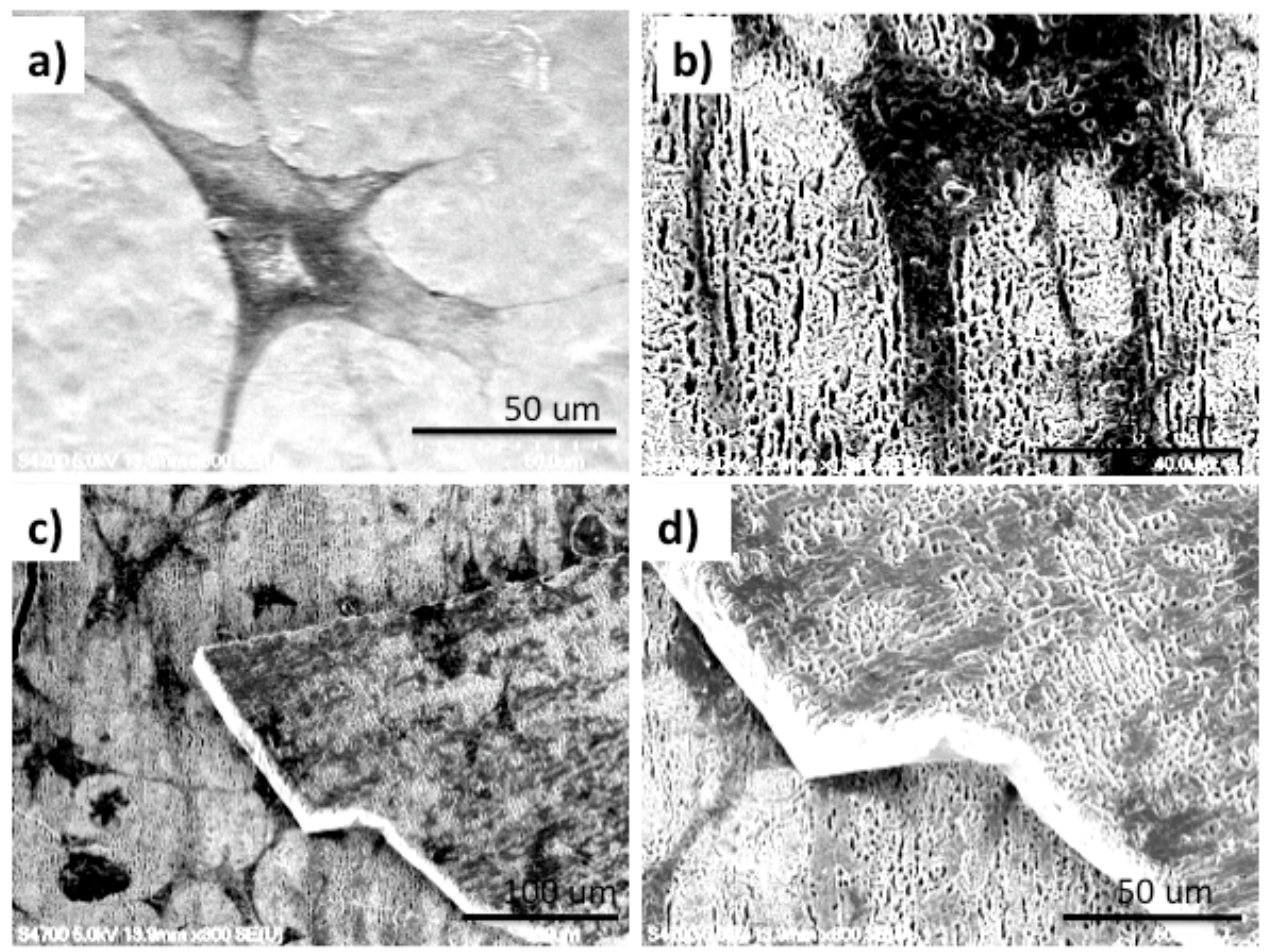

Figure 8.9. SEM micrographs of osteoblast cells when cultured on $\mathrm{TiO}_{2}$ nanotubes:

random cell orientation on control Ti sample without nanotubes (a) parallel cell orientation to the nanotube direction (b-c-d). Scale bare for (a-b-d) is $50 \mu \mathrm{m}$ and for (c) is $100 \mu \mathrm{m}$.

\subsection{FIB Milling Studies at Cell-Nanotube Interfaces}

Initially, fibroblast adhesion to $\mathrm{TiO}_{2}$ nanotubes was examined by the FIB method and then this work was extended to osteoblast cells. The main reason that I selected fibroblasts for the initial FIB work was that these cells make strong bonds with most substrate materials. In addition, in practical in-vivo application of biomedical implants fibroblast cells are the first to bond with the surface of the implant. The investigation on fibroblast cells was performed to demonstrate the potential of the FIB and SEM techniques on cell/biomaterial interface studies. Major attention was devoted to the feasibility and quality evaluation of different operations on air dried fibroblast cells as well as the interaction between the fibroblasts and $\mathrm{TiO}_{2}$ nanotubes. The results showed that fibroblast filopodia strongly interacted with the cell's nano-environment. Figure 8.10 shows FIB milled fibroblast cells obtained from a primary ion beam. Internal 
structures, membranes and morphological stability and integrity are shown. The interface between the cell and the substrate can be observed. Figure 8.11 shows the magnified cross sectional view of cell-nanotube interface. As shown in this image, after milling the cell by FIB for interface observation, the filopodia appeared to grow into the nanotubes. The results also revealed that fibroblats sustained very high vacuum without visible damage and they survived ion beam milling and imaging. Moreover, ion milling revealed many internal features of cells at the submicron scale (Figs. $8.11 \mathrm{a}-\mathrm{b}$ ) as well as the high interaction and growth of the cells inside the nanotubes. This showed that FIB and SEM, operating in high vacuum environment, can be applied on dried biological material that is not prepared following standard cryopreservation methods, glutaraldehyde or a combination of glutaraldehyde and formaldehyde procedures. ${ }^{144}$ Moreover it opens the path to the investigation on the physical basis of cell response to high vacuum conditions.

The FIB is preferably used on embedded biological samples, but I also successfully showed the use of FIB on biological samples prepared for conventional SEM. Such an approach can establish a link between larger scale tissue morphology and cellular and subcellular structures. The ability to reveal specific intracellular structural details and to link them to the interaction between cells and biomaterials is at the moment among the most promising and beneficial applications of FIB and SEM in life sciences. This is of particular interest when cells or cellular inclusions have a dynamic nature due to normal stress or pathologic conditions and their interaction with the biomaterial can significantly regulate their function. 

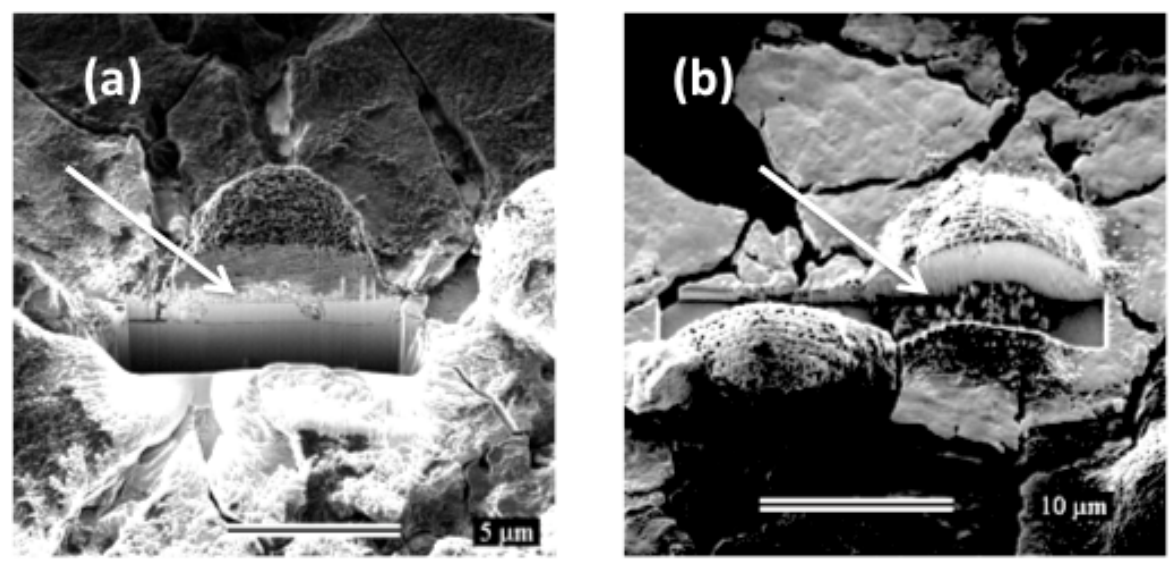

Figure 8.10. (a-b) FIB milled fibroblast cells obtained from a primary ion beam. Internal

structures, membranes and morphological stability and integrity can be assessed; moreover the interface between the cell and the substrate can be observed. Scale bar (a) is $5 \mu \mathrm{m}$, and (b) is $10 \mu \mathrm{m}$.
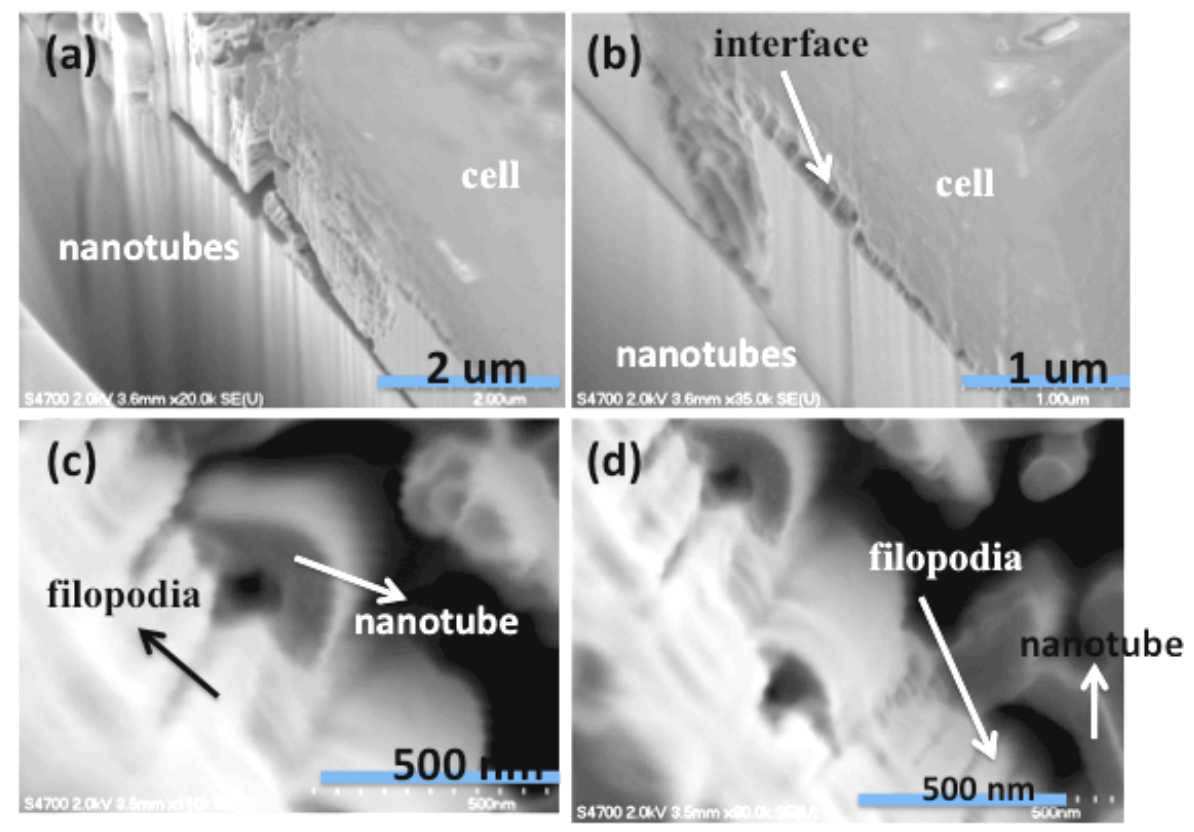

Figure 8.11. SEM images of the $(a, b)$ cross section view of cell-nanotube interface, milled by FIB technique and (c, d) magnified view of cell-nanotube interaction and filopodia growth inside nanotube. Scale bar (a) is $2 \mu \mathrm{m}$, (b) is $1 \mu \mathrm{m}$, and (c,d) is $500 \mathrm{~nm}$. 
The same FIB and SEM investigation of fibroblasts for osteoblasts to further extend the study on evaluating osteoblast cell attachment and surface interaction. The interaction of $\mathrm{MC} 3 \mathrm{~T} 3$ osteoblast cells with $\mathrm{TiO}_{2}$ nanotubes is shown in detail in Figures 8.12 and 8.13. SEM images in Figures 8.12b and $\mathrm{c}$ show the extension of osteoblast cells for adhesion on the nanotubes as well as cell clusters (Figure 8.12.a) on the nanotube surface which may indicate the ability of the nanotube structure to promote cell adhesion and growth (see Section 8.4). As can be seen in these images the surface tends to induce cell number, attachment and spreading. In Figure 8.12 the fully spread morphology of the osteoblast cells (Figure 8.12.a) as well as expansion of cell filopodia (Figure 8.12 b and c) can be observed. Figure 8.13 shows the attachment of cell filopodia to the nanotubes and filopodia expansion in between the nanotubes. From Figure 8.13 it is possible to think that the nanotubes can act as anchors for cell filopodia to grab (Figure 8.13f) and increase the spreading (Figure 8.13d) and improve its attachment (Figure 8.12).

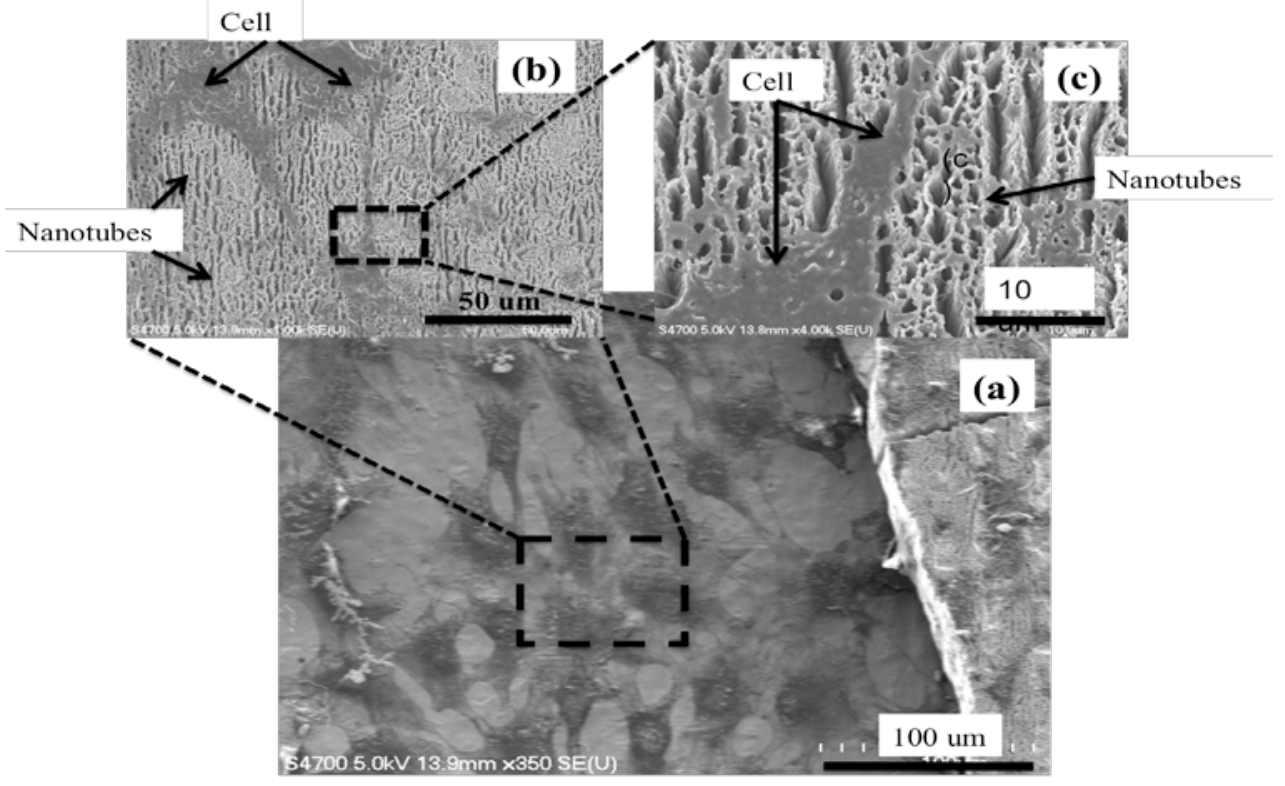

Figure 8.12. Osteoblast cell interaction with $\mathrm{TiO}_{2}$ nanotubes is shown. The extension of osteoblast cells for adhesion on the nanotubes as well as cell clusters (a) on the nanotube surface, indicate the ability of the nanotube structure to promote cell adhesion, and growth can be observed. Scale bar (a) is $100 \mu \mathrm{m}$, (b) is $50 \mu \mathrm{m}$, and (c) is $10 \mu \mathrm{m}$. 

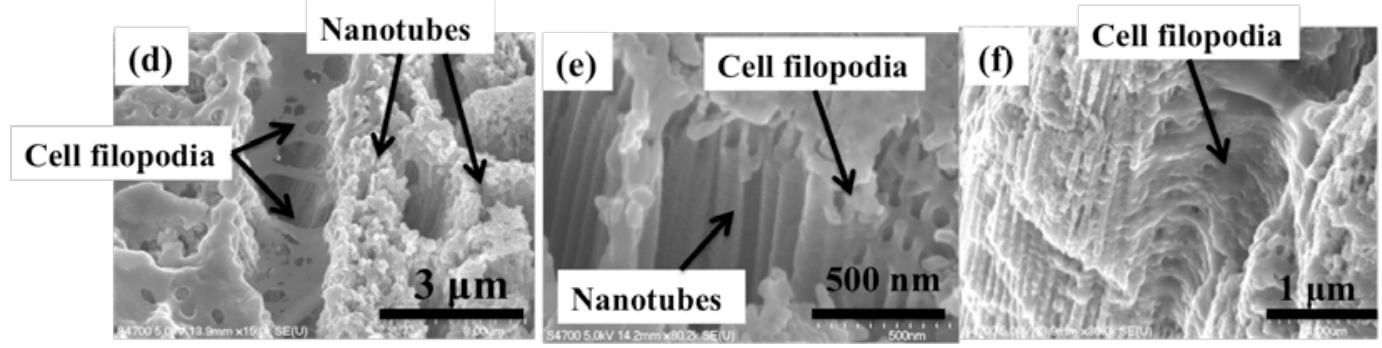

Figure 8.13. Cell filopodia attachment and spreading on and in between nanotubes. The nanotubes can act as anchors for cell filopodia to grab (f) and increase the spreading (d) and improve its attachment (e). Scale bar (d) is $3 \mu \mathrm{m}$, (e) is $500 \mathrm{~nm}$, and (f) is $1 \mu \mathrm{m}$.

The evaluations of cell anchorage and interlock were performed by FIB milling and SEM. The architecture of the cell/surface interface was visualized by electron microscopy to give a better insight into the morphological and functional features of interfacial osteoblast cell attachment. Figure 8.14 shows SEM images of the FIB milled cells reveal the nanotubes have been completely clogged by the cells. Even after removal of the cell top section by FIB (Figure 8.14.c and d) the nanotubes remain clogged (Figure $8.14 \mathrm{e}-\mathrm{f}$ ) approximately $5 \mathrm{~nm}$ in depth (Figure 8.15 ). This is due to high attachment of the cells, which has been qualitatively indicated from SEM images in Figures 8.12, 8.13 and 8.14A, indicating the well spread cell morphology and the cell growth inside the hollow section of the nanotubes (Figure 8.15). Figure 8.14 shows that the osteoblasts have direct cell/surface contact with the nanotubes and are firmly attached to the substrate surface. FIB processing by cell milling for the SEM investigations and EDS chemical analysis suggested that the bond between the $\mathrm{TiO}_{2}$ nanotube substrate and the adjacent osteoblast cell layer is composed of $\mathrm{Ca}$ and $\mathrm{P}$ elements which therefore seemed to mimic the bond in the bone tissue itself. Figure 8.14 and 8.15 indicate that on the substrate surface, cell filopodia and the extracellular matrix remained attached following separation from the milled top section of the cell. These observations reveal a very close interfacial contact or direct contact between the osteoblasts and the titania nanotubes followed by calcium and phosphorous deposition on the nanotubes (Figure 8.15). Calcium and phosphorous are the primary components of bone matrix and their deposition onto nanotubes, which is 
confirmed in Figure 8.15, is an indication that $\mathrm{TiO}_{2}$ nanotubes have regulated osteoblast functionality and differentiation.
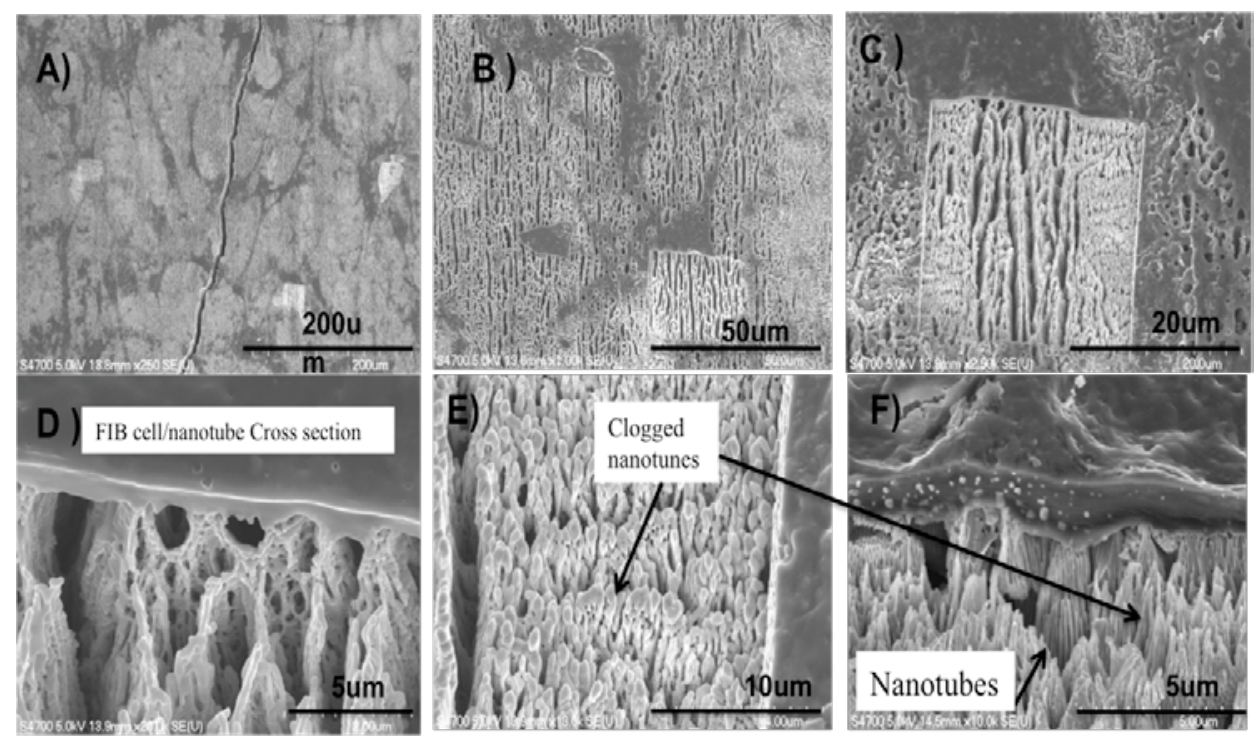

Figure 8.14. FIB cross sectional milling (A-D) revealing the clogged titania nanotube by osteoblast and calcium deposition on nanotubes (F-F) as a result of cell attachment and direct contact with surface. Scale bar (A) is $200 \mu \mathrm{m},(B)$ is $50 \mu \mathrm{m},(\mathrm{C})$ is $20 \mu \mathrm{m},(\mathrm{D}, \mathrm{F})$ is $5 \mu \mathrm{m}$, and $(\mathrm{E})$ is $10 \mu \mathrm{m}$.

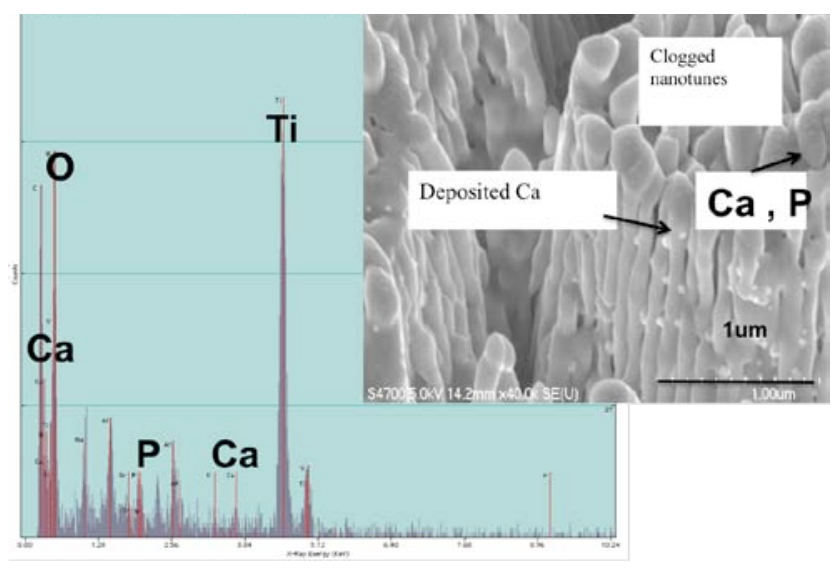

Figure 8.15. EDS analysis of the high-resolution scanning electron micrograph of clogged nanotubes. The clogged nanotubes are visible and composed of $\mathrm{Ca}$ and $\mathrm{P}$ (EDS), the primary components of bone matrix. Scale bar is $1 \mu \mathrm{m}$. 
The SEM images in Figure 8.16 show the secreted extracellular matrix (ECM) all over the surface of the sample. The cellular activity or differentiation of osteoblasts on the surface initiates by deposition of calcium and phosphorous as primary components of bone matrix. ${ }^{145}$ As shown in Figure 8.16 this production of $\mathrm{ECM}$ on $\mathrm{TiO}_{2}$ nanotubes is the result of osteoblast migration and its healthy cellular activity. In fact as shown earlier in Figures 8.12 and 8.13, it is possible to consider the $\mathrm{TiO}_{2}$ nanotubes as anchors for the cell filopodia to grab onto and have a facilitated migration along the surface. This anchorage benefit of nanotubes together with their super hydrophilic properties appears to have encouraged osteoblast number, spreading and attachment for a promising high osseointegration.
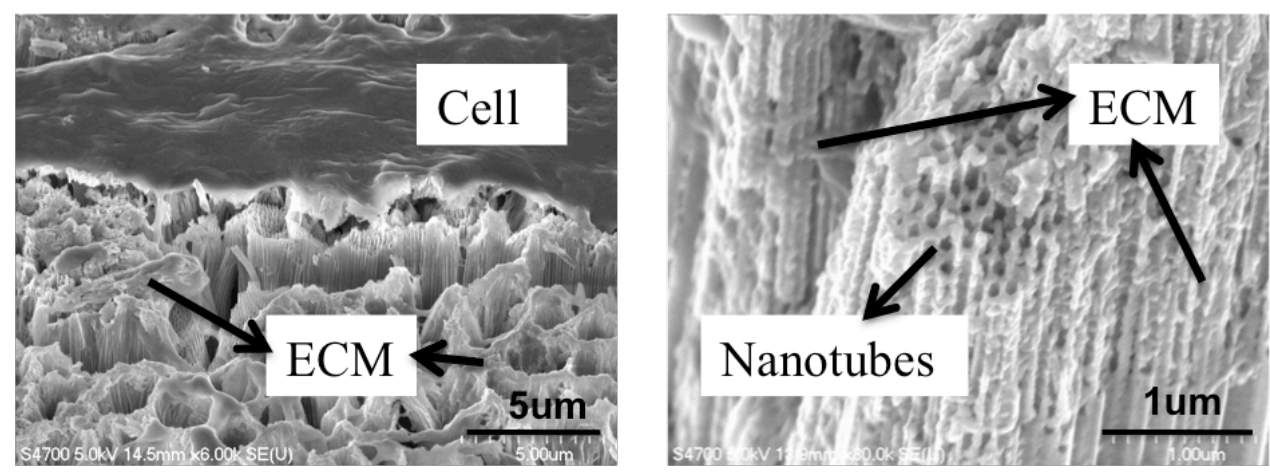

Figure 8.16. ECM production on and in between nanotubes during cell migration and bone matrix deposition. Scale bar left is $5 \mu \mathrm{m}$ and right is $1 \mu \mathrm{m}$.

Oh et al. ${ }^{146}$ investigated the effect of titanium nanotubes with different crystallographic structures on MC3T3-E1 osteoblast cells. They concluded that the cell filopodia grew inside the nanotubes, making a strong interlock between the cell and substrate. However, their discussion on interlocked interactions between the nanotubes and cell filopodia is not conclusive enough due to lack of any structural evidence. Here, we have provided evidence of such an interlocked mechanism by means of FIB technique for the first time.

\subsection{Effect of Nanotube Stiffness on Cell Number}

One of the major advantages of nanotubular morphology over the surface 
of implants is the ability to calculate and measure the extracellular forces exerted by the cells that grow on top of the surface. This tool allows a quantitative assessment of the adhesion between cells and the surface, which are addressed in Chapter 10 as one of the extensions of this $\mathrm{PhD}$ work. However, I have used this advantage to propose a method for quantifying the relationship between the nanotube stiffness and osteoblast cell number. In fact, the geometry of $\mathrm{TiO}_{2}$ nanotubes on the surface of $\mathrm{Ti}$ implants can be modeled as a series of clamped cantilevers with bending stiffness $K$ subjected to a shear force at the free end.

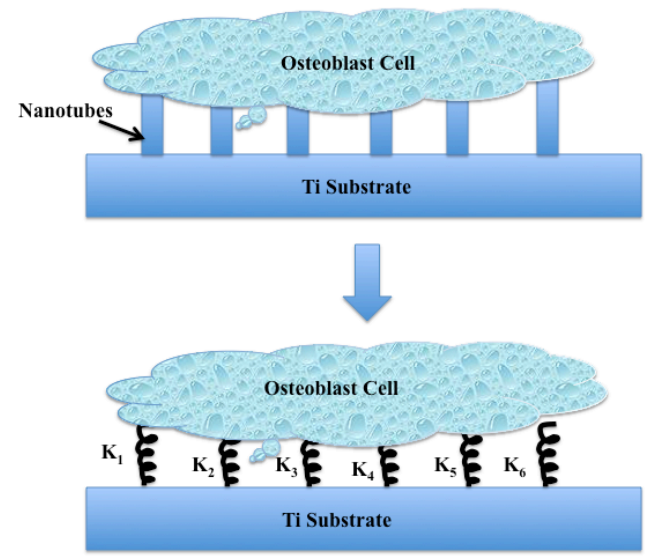

Figure 8.17. The vertically-aligned $\mathrm{TiO}_{2}$ nanotubes that are anodized on the Ti implant surface can be modeled as a series of cantilever springs with spring constants of $\mathrm{K}_{1}, \mathrm{~K}_{2}$,

$$
\mathrm{K}_{3}, \ldots \mathrm{K}_{\mathrm{n}} \text {. }
$$

In practice, a nanotube can be bent by the adhesion forces exerted by the osteoblast cells. According to beam theory, ${ }^{147}$ for a cantilever under a bending force $F$, the small deflection $\delta$ at the free end have a relationship with the applied force $F$ as

$$
\delta=F L^{3} / 3 E I
$$

where $L$ is the length of the nanotube, and $E$ is the elastic modulus. The moment of inertia is:

$$
I=\pi / 4\left(d_{0}{ }^{4}-d_{i}{ }^{4}\right)
$$

By assuming a circular cross section for the nanotubes, the parameters $d_{0}$ and $d_{i}$ represent the outer and inner diameters of the nanotubes, respectively. The elastic 
modulus of the nanotubes was measured to be in the range of 23-44 GPa depending on the diameter of the nanotubes. ${ }^{148}$ Here I have defined a normalized-stiffness parameter, $K_{a}$ as:

$$
K_{a}=F / \delta=3 E I / L^{3} \alpha I / L^{3}
$$

To quantify the nanotube stiffness variation in terms of the nanotube length, the parameter $K_{a}$ was calculated for various nanotubes obtained under different anodization voltage and the data is plotted. As seen in Figures 8.18 and 8.19 the stiffness of the nanotubes decreases by the increase in the nanotube length (Figure 8.18) and anodization time (Figure 8.19). This observation was consistent for nanotubes produced with 20, 40, and $60 \mathrm{~V}$.

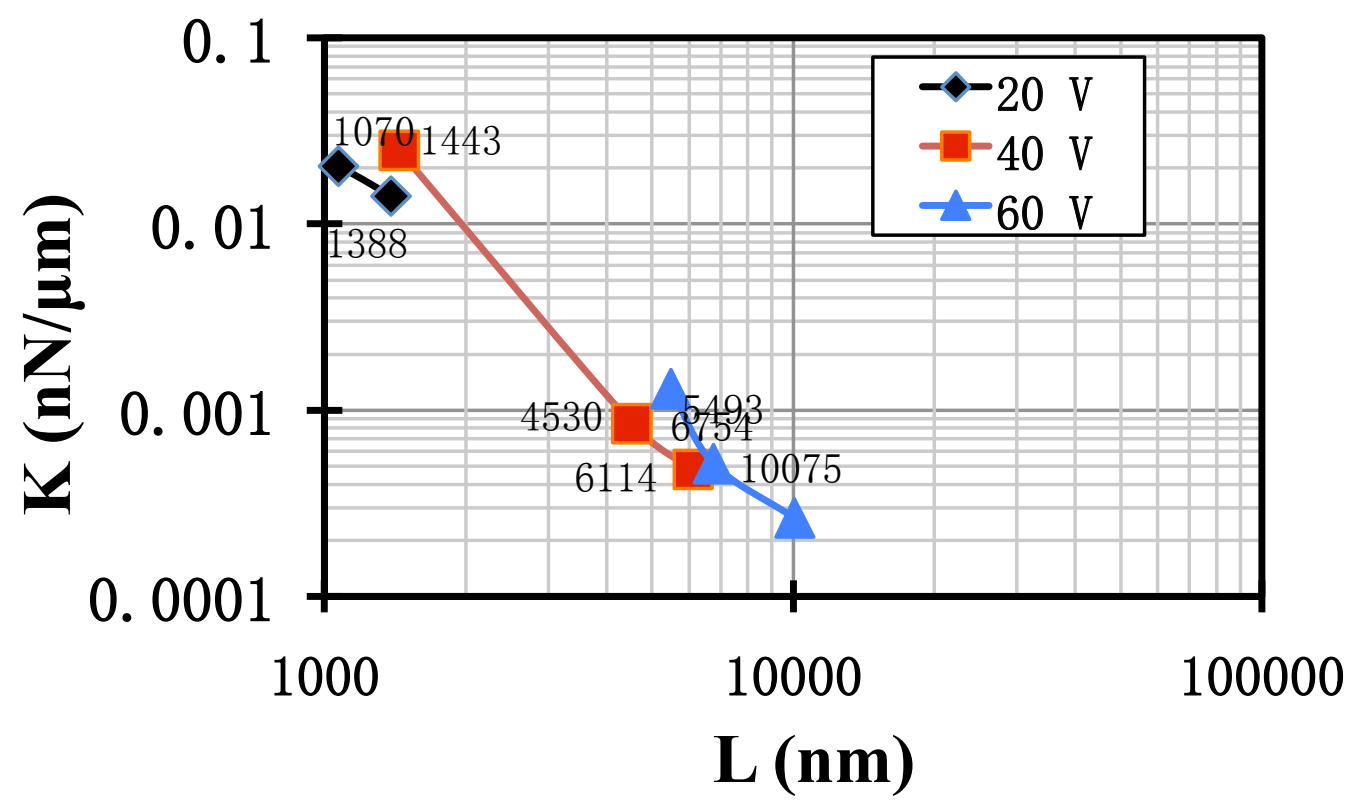

Figure 8.18. The normalized $K a$ parameter as a function of nanotube length, $L$, is plotted. The nanotubes were synthesized at three different applied voltages, 20, 40, and $60 \mathrm{~V}$. 


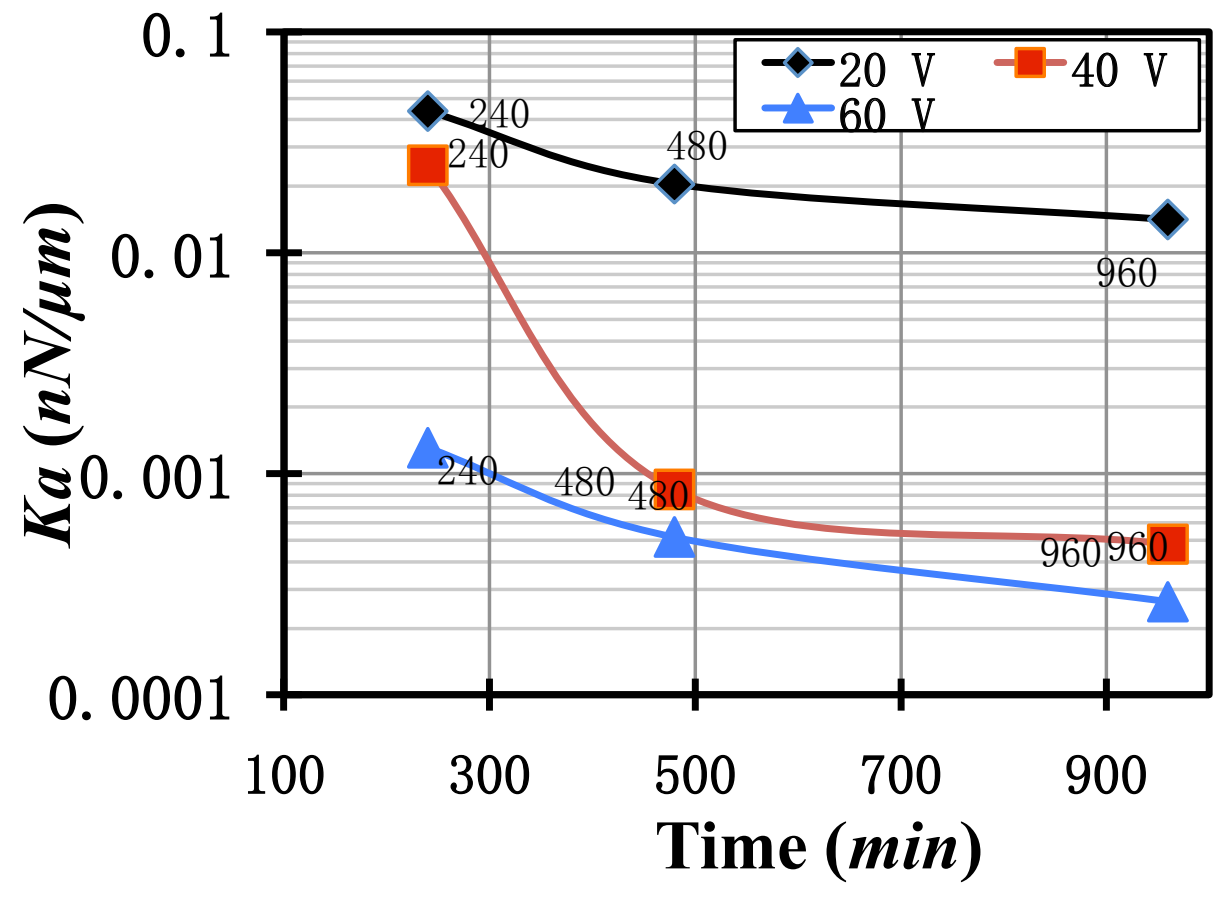

Figure 8.19. The normalized $K a$ parameter as a function of anodization duration, $T$, is plotted. The nanotubes were synthesized at three different applied voltages, 20, 40, and $60 \mathrm{~V}$.

As discussed earlier in Section 8.4, the literature shows that nanotube diameter affects cell number. However, there is lack of data on the effect of nanotube length on cell growth. Here, I have shown that not only diameter but also nanotube length apparently affects cell number. The results indicate that nanotubes with the same crystalline structure and chemical composition but higher anodization duration (and consequently higher length and diameter) show a decrease in the number of attached cells. As seen in Figure 8.8, in the case of amorphous (as anodized) cp-titania nanotubes, the anodization duration of $240 \mathrm{~min}$ shows a higher number of attached cells (1500 counts $\left./ \mathrm{mm}^{2}\right)$ in comparison to nanotubes anodized for $960 \min \left(1100\right.$ counts $\left./ \mathrm{mm}^{2}\right)$. This finding signifies the importance of considering both the diameter and length of nanotubes on cell number and attachment. As discussed in this section nanotube length and diameter, both are the main parameters of nanotube stiffness. Therefore, I believe that the main reason for the nanotube geometric effect (diameter and length) on osteoblast 
cell number and attachment is the change in stiffness of nanotubes. To study this effect only one parameter (anodization duration) can be changed and other factors, such as annealing and composition should remain constant. Therefore, the effect of nanotube stiffness on cell number for as-anodized alloyed $\mathrm{TiO}_{2}$ nanotubes was studied. This relationship between nanotube stiffness and cell number is illustrated in Figure 8.20. As seen in this figure, the nanotubes with higher stiffness $\left(\mathrm{k}=13.07 \times 10^{-4} \mathrm{nN} / \mu \mathrm{m}\right)$ promote more cells (up to 800 cells $/ \mathrm{mm}^{2}$ ) in comparison to nanotubes with lower stiffness $(\mathrm{k}=$ $\left.2.6 \times 10^{-4} \mathrm{nN} / \mu \mathrm{m}\right)$ and 300 cells $/ \mathrm{mm}^{2}$. This finding is in agreement with the work of Khatiwala et al. ${ }^{149}$ who concluded that substrate stiffness influences focal adhesion formation, migration, number, and, differentiation, of mouse osteoblastic cell line (MC3T3-E1) towards an osteoblastic phenotype as early as 4 days. ${ }^{150}$ Over the last decade several reports have shown that substrate rigidity and stiffness can control various functions of a healthy cell, such as migration, number, and differentiation. ${ }^{151,152,153}$ The same process may also explain why, during tissue formation, cells tend to maximize their net area of adhesion to one another while minimizing the area exposed to the surrounding environment. These observations are relevant not only to tissue formation during development but also a number of important physiological and pathological processes. For example, cell migration during wound healing may be guided by the contractile forces at the wound, whereas cancer metastasis may be caused by cells losing the ability to respond to the differential mechanical signals between the tissue and the membrane. 


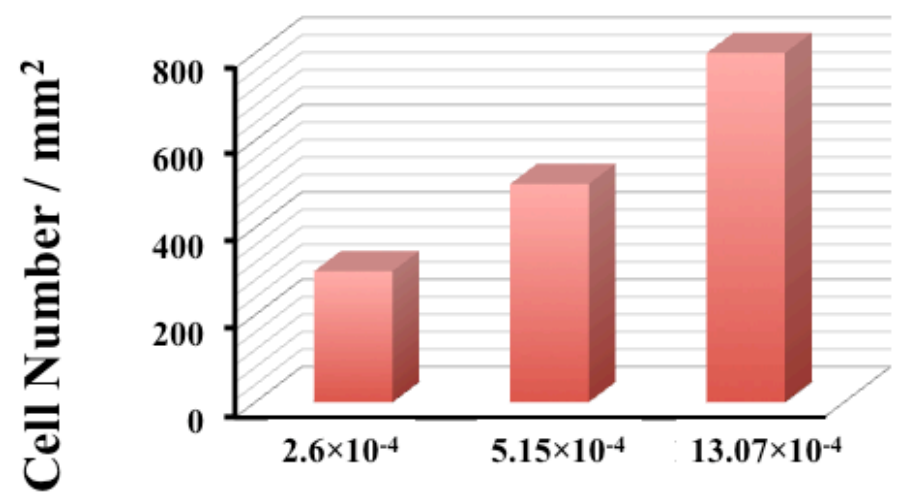

\section{Normalized Stiffness of Nanotubes $(\mathrm{nN} / \mu \mathrm{m})$}

Figure 8.20. Effect of nanotube stiffness on cell number is plotted for $\mathrm{TiO}_{2}$ nanotubes anodized at $60 \mathrm{~V}$. The higher the stiffness, the better the cell growth.

In a study by Pelham et al. ${ }^{154}$ responses of rat $3 \mathrm{~T} 3$ fibroblastic cells to mechanical properties of the adhesion substrate were examined. Compared with cells on rigid substrates, those on flexible substrates showed irregularly shaped focal adhesions whereas those on firm substrates had a normal morphology and were much more stable. In another report, the migration speed of mouse 3T3 fibroblasts was shown to increase proportionally to the rigidity of the substrate. ${ }^{155}$ In addition, rigid surfaces enhanced the proliferative and reduced the anti-apoptotic potential of $3 \mathrm{~T} 3$ fibroblasts compared to the more compliant substrate conditions. ${ }^{156}$ This behavior of aggregation mimicking tissue formation may be driven at least partially by durotaxis, the preferential migration of cells toward stiff substrates or away from soft substrates. ${ }^{157}$ Furthermore fibroblasts are able to actively probe the stiffness of their environment and to turn toward substrates of high rigidity, as durotaxis. This surface tension-like mechanism is believed to also drive cell sorting. ${ }^{158}$ Other studies have also shown that, rigid surfaces enhance the proliferative and reduced the anti-apoptotic potential of 3T3 fibroblasts compared to the more compliant substrate conditions. ${ }^{159,160}$ Further studies on tissue engineering ${ }^{161}$ support this idea that substrate stiffness regulates not only cell migration and adhesion but also cell growth and 
apoptosis ${ }^{162}$, which are closely related to cell shape and adhesion and are equally important in tissue formation.

The general principle underlying these observations appears to be a preference of cells to maximize mechanical input from the environment. How could cells sense the difference among substrates of identical chemical properties but varying flexibilities and elastic modulus? To detect flexibility, it is necessary for the cells to modulate and measure the probing force in response to different substrate resistance (otherwise, cells will simply deform soft substrates to an increasing extent until they experience a similar resistance as on stiff substrates). Alternatively, cells may be able to sense the amount of substrate deformation as they apply a defined probing force. For this purpose, cells must rely on an active probing mechanism using contractile forces. ${ }^{163}$ Mechanical input from the substrate, transmitted through adhesion receptors, then activates downstream signals that regulate both the degree of cell spreading and the rate of growth. On stiff substrates, resistance to mechanical probing may lead to protein conformational changes and activation of signaling enzymes at the adhesion sites. The response in turn causes an increase in traction forces and in cell spreading. ${ }^{164}$

The understanding of cell active force probing mechanism can help us explain my results based on the fact that it is easier for the cells to use the stiffer nanotubes as anchoring steps for their attachment and migration. Cell filopodia can use the nanotubes for anchoring and then contracting to move the cell forward. Mechanical input from the substrate is transmitted through adhesion receptors, and then it activates downstream signals that regulate both the degree of cell spreading and the rate of growth. Since the stiffer nanotubes can promote more mechanical output for the probing cells therefore more mechanical input from nanotubes will be transmitted through adhesion receptors and more signals for cell number will be activated. These phenomena will finally results in higher cell number on stiffer nanotubes. 


\section{Chapter 9}

\section{Discussion and Conclusions}

This chapter provides an overview to the most significant results of my $\mathrm{PhD}$ research work. Initially, I worked on the surface modification of Ti4A16V alloys through porosification via hydrofluoric acid. Several etching techniques were compared including a method based on sandblasting as a pretreatment. It was found that the increase of applied voltage and concentration of $\mathrm{H}_{3} \mathrm{PO}_{4}$, increased the pore sizes at the surface of titanium oxide. During this electrochemical etching, between these two parameters, the applied voltage is the main parameter that controls the morphology of porous structure in comparison to the time of etching. The selective electro-etched (SEE) process was conducted in machined $\mathrm{Ti}$ surfaces (no significant roughness) and resulted in small porous structure where the maximum pore sizes were about $\sim 1-3$ microns. The sandblasted and acid-etched surface (SLA) possessed a combination of micro porosity and roughness. The SLA process resulted in large pore sizes in the range of 10-30 microns. In contrast, the sand blasted and electro etched (SLE) surfaces were shown to contain a combination of micro/nano porosity. In this process, in addition to the large porous structure of 10-30 microns, nanopores with an average size of $500 \mathrm{~nm}$ were observed. It is expected that the SLE process is more desirable for implant application of titanium alloys due to the formation of such nanostructures. Therefore, the SLE process could be useful for bone and dental implants. However the process required the use of hazardous; expensive materials. In comparison between the SLA and SLE processes, it was observed that the population of nanopores decreased while the average pore size increased with an increase of the etching voltage. The acid etching technique has several limitations including working with hazardous acids, long times required for etching, and lack of control over the size distribution of pores.

Due to the above limitations, I focused on a more efficient technique to modify the surface of titanium alloys. Electrochemical synthesis of $\mathrm{TiO}_{2}$ nanotubes was 
conducted at the surface of $\mathrm{Ti}$ and $\mathrm{Ti} 6 \mathrm{Al} 4 \mathrm{~V}$ using a $\mathrm{NH}_{4} \mathrm{~F}$ in ethylene glycol electrolyte. The final morphology resembled vertically aligned nanotubes all over the titanium surface. This method does not require working with hazardous acids such as HF. In turn, the sizes of the nanotubes can be controlled to a great extent by adjusting the synthesis parameters of applied voltage, time, and chemistry of the electrolyte. It was observed that the nanotube diameter increased over time. Moreover an increase in voltage resulted in enhanced nanotube diameter and length. After $4 \mathrm{hrs}$ of anodization the increase in voltage values from $20 \mathrm{~V}$ to $40 \mathrm{~V}$ and $60 \mathrm{~V}$ resulted in enlarged nanotube diameters from $36 \mathrm{~nm}$ to $66 \mathrm{~nm}$ and $81 \mathrm{~nm}$ as well as increased nanotube length from $589 \mathrm{~nm}$ to $1443 \mathrm{~nm}$ and $5493 \mathrm{~nm}$ respectively. In $8 \mathrm{hrs}$ of anodization with voltage increasing from $20 \mathrm{~V}$ to $40 \mathrm{~V}$ and $60 \mathrm{~V}$ the nanotube diameter enlarged from $48 \mathrm{~nm}$ to $67 \mathrm{~nm}$ and $113 \mathrm{~nm}$ and the nanotube length increased from $1070 \mathrm{~nm}$ to $4530 \mathrm{~nm}$ and $6754 \mathrm{~nm}$ respectively. Finally at $16 \mathrm{hrs}$ of anodization with voltage increasing from $20 \mathrm{~V}$ to $40 \mathrm{~V}$ and $60 \mathrm{~V}$ the nanotube diameter enlarged from $54 \mathrm{~nm}$ to $74 \mathrm{~nm}$ and $144 \mathrm{~nm}$ and the nanotube length increased from $1388 \mathrm{~nm}$ to $6114 \mathrm{~nm}$ and $10075 \mathrm{~nm}$ respectively.

To use these nanomaterials in clinical applications, one should know the mechanical, electrical, structural stability, and surface hydrophilicity properties which play an important role in the biocompatibility of the nanotubes. The mechanics of the nanotubes is important from stress-shielding and mechano-trunsduction points of view. Interestingly, no previous reports in the literature had been conducted on the mechanical properties of nanotubes. Novel experiments were conducted on measuring the mechanical properties of individual $\mathrm{TiO}_{2}$ nanotubes. The nanotubes were categorized as thin and thick walls. The thin-wall tube had external and internal nominal diameters of $\sim 75$ and $\sim 65 \mathrm{~nm}$. The thick-wall tube had external and internal nominal diameters of $\sim 110 \mathrm{~nm}$ and

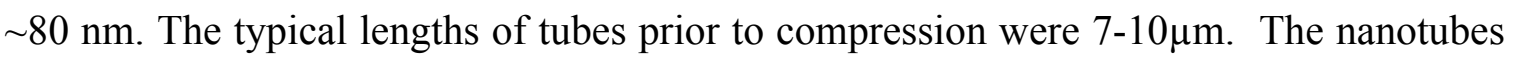
were tested in the chamber of a transmission electron microscope using an in-situ atomic force microscopy stage with force resolutions better than $1 \mathrm{nN}$. Both thin and thick nanotubes were tested to understand the variations of elastic properties as a function of size. The in-situ TEM studies allowed us to correlate the load-displacement measurements with changes in the tubes' structure. During compressive loading, a 
phenomenon was observed that corresponded to a drop of force on the thin-wall tube. The force drop was correlated to the collapse of nanotubes due to the compressive forces.

The collapse of thin-wall nanotubes could be due to localized deformation and failure originated by nanoscale defects. The TEM imaging showed that the nanotube surfaces contained irregularities and this may have caused stress concentration and failure. The measured compressive forces ranged between $1.2 \mu \mathrm{N}$ and $7 \mu \mathrm{N}$. These forces, coupled with the observed $\mathrm{TiO}_{2}$ nanotube dimensions, corresponded to compressive stresses up to $1564 \mathrm{MPa}$ and lead to a Young's modulus of $\mathrm{TiO}_{2}$ nanotubes in the range of $23 \mathrm{GPa}$ to $44 \mathrm{GPa}$. Beacause the compressive elastic behavior was size dependent, it is important to note that this is not interpreted as a constant material property. The surface modification of $\mathrm{Ti}$ implants by a $\mathrm{TiO}_{2}$ nanotube layer with elastic modulus value close to the actual bone should, in principal, promote the osteoblast cell and bone growth and eventually could be a promising avenue to overcome stressshielding, a common reason for implant failures.

The electrical properties of $\mathrm{TiO}_{2}$ nanotubes was next studied. My motivation was based on the work of Ercan et al. ${ }^{165}$ which showed that application of the electrical signaling on anodized nanotubular titanium can significantly improve the osteoblast adhesion, proliferation, and differentiation However, no reports in the literature, on the electrical properties of individual nanotubes were found. This need can be realized if one notes that the biological cells are normally in nano size and have interactions with nanotubes at the nanoscale. This interaction can result in bending or straining of the nanotubes. I successfully carried out in-situ electrical transport measurements on individual anatase and amorphous $\mathrm{TiO}_{2}$ nanotubes inside a high-resolution transmission electron microscope equipped with the scanning tunneling microscope (STM) probe. The amorphous nanotubes showed almost insulating behavior, while the crystalline nanotubes behaved like a semiconductor (non-linear I-V curves). It was shown for the first time that the electrical transport properties of the amorphous $\mathrm{TiO}_{2}$ nanotube could be tuned from the insulating to semiconducting by inducing deformation. Also, the conductivity of the crystalline (anatase) nanotubes increased as a function of mechanical straining. The semiconducting parameters were retrieved from the experimental current-voltage (I- 
V) curves using the Metal-Semiconductor-Metal (M-S-M) model. The nanotubes under the higher deformation had approximately 2.2-times higher charge carrier density but $\sim 1.74$ times lower charge mobility. Such phenomena can be related to the strain engineering of the electronic band gap structure in nanotubes. When the anatase $\mathrm{TiO}_{2}$ nanotubes are brought into the deformed state, in-shell defects are produced at the walls of the nanotube. The possible defects can be voids, vacancies, and antisite atoms, which modify the band structure.

The next study was related to the effect of a nitrogen-enriched environment and temperatures (annealing treatment) on the structural properties of the nanotubes. This is motivated by the fact that during the process of implant fabrication there maybe industrial treatments that require annealing or treating the implant in a nitrogen concentrated environment. Moreover, It has been shown that, upon exposure to UV light, $\mathrm{TiO}_{2}$ nanotubes exhibit very high photocatalytic efficiency that can be used for the decomposition of organic compounds in the contact mode (on the Ti substrate). However to achieve this desired purpose a direct access of the light to the $\mathrm{TiO}_{2}$ nanotubes is required. It has been suggested that $\mathrm{N}$-doped material may be used to trigger a photocatalytic reaction on $\mathrm{TiO}_{2}$, for obtaining effects at longer wavelength with higher penetration depth. This can be of high importance for a sterilization procedure under UV light, as well as potential application of $\mathrm{TiO}_{2}$ nanotubes for cancer therapy. ${ }^{166}$

During the process of amorphous to anatase transformation in annealing the asanodized nanotubes, the length and average diameter of the nanotubes had no discernible changes. It has been suggested that the initial crystallization of anatase took place both in the nanotubes and at the nanotube-foil interface at almost the same temperature. In this process, some of the closely spaced anatase crystallites coalesced to form larger crystallites. The nanotubes treated in $\mathrm{NH}_{4} \mathrm{OH}$ solution prior to annealing showed drastic differences in comparison to the non-treated nanotubes. The $\mathrm{NH}_{4} \mathrm{OH}$ treated nanotubes collapsed into nanoparticles after a few minutes of exposure to high temperatures. It was concluded that $\mathrm{NH}_{4} \mathrm{OH}$ treatment of $\mathrm{TiO}_{2}$ nanotubes resulted in the incorporation of nitrogen in surface roughness and eventually the weakening of the nanotubes. The treatment of nanotubes in $\mathrm{NH}_{4} \mathrm{OH}$ solution and subsequent annealing at $500^{\circ} \mathrm{C}$ 
resulted in structural transformation to anatase nanoparticles and the associated volumetric change resulted in high stress concentrations at the surface roughness areas. The nanotubes barely maintained their tubular morphology after $30 \mathrm{~min}$ of treatment. After this, the nanotubes collapsed into nanoparticles while the transformation to anatase happened simultaneously. These results contradicted the common belief that the transformation to rutile was the major reason for the collapse of anatase nanotubes.

Next, the effect of electrical currents (Joule heating) on the properties of nanotubes was studied. The experiments were conducted in TEM using the in-situ STM holder and the structural transitions were recorded. Under large currents, the nanotubular structure collapsed into small particles $(\sim 10-20 \mathrm{~nm})$. These nanoparticles were rutile particles and formed directly after the dissociation of anatase nanotubes. The size of the rutile nanoparticles was controlled by the applied bias voltage. The anatase nanotubes under Joule heating dissociated to small particles of anatase at low bias voltage $(\leq 10 \mathrm{~V})$. At intermediate bias range, between $10 \mathrm{~V}$ and $20 \mathrm{~V}$, the anatase nanoparticles transformed to rutile. Under the bias condition of $30 \mathrm{~V}$, the rutile particles agglomerated into large particles with sizes that ranged up to $200 \mathrm{~nm}$. It is known that thermodynamic stability of different phases of $\mathrm{TiO}_{2}$ is size dependent and thus by controlling the size of the particles, we can control the properties of the $\mathrm{TiO}_{2}$ used for various applications. This result opens new discussions and future efforts on what regime the electrical methods for regulating cell functions can be effective before damaging the nanotube architectures.

Next the surface wettability properties of nanotubes were studied. The surface characteristics are important since after implantation, the biological interaction of the biomaterial with cells initially occurs in the surface of the implant. This study examined the wettability of bare titanium and alloy, and $\mathrm{TiO}_{2}$ nanotube (TNT) structured and annealed and aged surfaces by analyzing the contact angles with $1 \mu$ de-ionized water droplets. From the gathered data, three conclusions were drawn. First, the TNT surfaces had a hydrophilic characteristic caused by the anodizing process and a surface initially with many hydroxyl groups instead of an oxide. As the chemical analysis indicated, and considering the degree of electrolytic dissociation, the surfaces were initially composed of $\mathrm{Ti}(\mathrm{OH})_{4}$ groups instead of $\mathrm{TiO}_{2}$ resulting in lower contact angles. Second, 
as a result of annealing the TNT surfaces became super-hydrophilic apparently due to the generation of anatase. Extensive anodization can cause delamination of the TNT layer resulting in irregular surface roughness that can locally affect the contact angle. Third, aging of TNT surfaces led to a marked decrease of hydrophilicity. This is the result of the stabilization process for the substitution reaction from $\mathrm{Ti}(\mathrm{OH})_{4}$ to $\mathrm{TiO}_{2}$ as the hydroxyl groups disappear in air. These results indicated that where surface wettability may be an advantage, nanotube growth by anodization using the process described here can satisfy this requirement. This may have extensive application for titanium orthopedic implants where the wettability characteristics of the surface is specified.

I further investigated the effect of chemical composition, crystallography, and stiffness of $\mathrm{TiO}_{2}$ nanotubes on osteoblast cell function. First an initial study on the cell shape was conducted to evaluate the morphological changes in osteoblast cells during the attachment period. Over the course of $24 \mathrm{hrs}$ it was observed that the cells changed their shape in a healthy way from round and not attached to fully spread and completely attached cells.

Then the osteoblast cells were cultured on control cp-Ti and amorphous cp- $\mathrm{TiO}_{2}$ nanotubes as a function of incubation time to investigate the viability of nanotubes and their effectiveness on cell growth compared to conventional plain surfaces. I observed that, on control cp-Ti substrates cell count increased from 40 cell $/ \mathrm{mm}^{2}$ after $24 \mathrm{hrs}$ to 300 cells $/ \mathrm{mm}^{2}$ after $72 \mathrm{hrs}$ of incubation. However, the in-vitro results with nanotubes indicated that the nanotube morphology enhanced cell proliferation from 80 cells $/ \mathrm{mm}^{2}$ after $24 \mathrm{hrs}$ to 900 cells $/ \mathrm{mm}^{2}$ after $72 \mathrm{hrs}$ of incubation. I also investigated the effect of chemical composition on osteoblast growth and adhesion by comparison between alloyed and pure Ti. On the control Ti6Al4V substrate, the cell count increased from 12 cells $/ \mathrm{mm}^{2}$ after $24 \mathrm{hrs}$ to 200 cells $/ \mathrm{mm}^{2}$ after $72 \mathrm{hrs}$ of incubation However, the in-vitro results with alloyed nanotubes, indicated that the alloyed nanotube morphology enhanced cell proliferation from 40 cells $/ \mathrm{mm}^{2}$ after $24 \mathrm{hrs}$ to 800 cells $/ \mathrm{mm}^{2}$ after $72 \mathrm{hrs}$ of incubation. This enhanced cell proliferation is in agreement with the results on cp-Ti and cp- $\mathrm{TiO}_{2}$ nanotubes. However, the total cell counts on the control Ti6Al4V and alloyed$\mathrm{TiO}_{2}$ nanotubes was slightly lower for all incubation periods, in comparison to the 
total cell count in cp-Ti and cp- $\mathrm{TiO}_{2}$ nanotubes. I believe that this could be due to lower biocompatibility of the alloying elements, $\mathrm{Al}$ and $\mathrm{V}$, and their oxides.

In regard to the effect of nanotube crystallinity on cell growth, it was observed that after $72 \mathrm{hrs}$ of incubation, the cell number increased from 800 cells $/ \mathrm{mm}^{2}$ on amorphous (as-anodized) alloyed nanotubes to 2000 cells $/ \mathrm{mm}^{2}$ on annealed alloyed nanotubes and from 900 cells $/ \mathrm{mm}^{2}$ on amorphous cp-nanotubes to 2500 cells $/ \mathrm{mm}^{2}$ on annealed cp-nanotubes. However, similar to the control Ti6Al4V and alloyed- $\mathrm{TiO}_{2}$ nanotubes, the total cell counts on annealed alloyed $\mathrm{TiO}_{2}$ nanotubes was also slightly lower for all incubation periods, in comparison to the total cell count on annealed cp$\mathrm{TiO}_{2}$ nanotubes. This could be due to lower biocompatibility of alloying elements $\mathrm{Al}$ and V. It should be noted that in addition to nanotopography and crystallinity, higher surface hydrophilicity has resulted in enhanced osteoblast cell number on annealed $\mathrm{TiO}_{2}$ nanotube substrates compared to plain Ti substrates.

In addition, the osteoblast interaction and attachment with $\mathrm{TiO}_{2}$ nanotubes was investigated using focused ion beam milling. The SEM images of the FIB milled cells revealed that the top of the nanotubes was completely clogged by the cells. Even after removal of the cell top section by FIB the nanotubes remained clogged approximately 5 $\mathrm{nm}$ in depth, due to high attachment of the cells and the cell growth inside the hollow section of the nanotubes. In addition it was observed that the osteoblasts had direct cell/surface contact with the nanotubes and were firmly attached to the substrate surface, indicating the filopodia. EDS chemical analysis suggested that the bond between the $\mathrm{TiO}_{2}$ nanotube substrate and the adjacent osteoblast cell layer was composed of $\mathrm{Ca}$ and $\mathrm{P}$ elements which therefore seemed to mimic the bond in the bone tissue itself. These observations revealed a very close interfacial contact or truly direct contact between the osteoblasts and the titania nanotubes followed by calcium and phosphorous deposition on the nanotubes. Calcium and phosphorous are the primary components of bone matrix and their deposition onto nanotubes is an indication that $\mathrm{TiO}_{2}$ nanotubes have regulated osteoblast functionality and differentiation. Moreover I could observe the secreted extracellular matrix (ECM) all over the surface of the sample from the SEM images. The cellular activity or differentiation of osteoblasts on the surface initiates by 
deposition of calcium and phosphorous as the primary components of bone matrix. This production of $\mathrm{ECM}$ on $\mathrm{TiO}_{2}$ nanotubes was apparently the result of osteoblast migration and healthy cellular activity. In fact it is possible to consider the $\mathrm{TiO}_{2}$ nanotubes act as anchors for the cell filopodia to grab onto and have a facilitated migration along the surface. This anchorage benefit of nanotubes together with their super hydrophilic properties appears to have encouraged osteoblast proliferation, spreading and attachment for a promising high osseointegration.

Finally I proposed a method for evaluating the effect of nanotube stiffness on cell number and therefore a means to optimize the morphological properties of the nanotubes for increase in cell number. As an extension of this $\mathrm{PhD}$ work, one of the major advantages of nanotubular morphology over the surface of implants is the ability to calculate and measure the extracellular forces exerted by the cells that grow on top of the surface. Such morphology allows a quantitative assessment of the adhesion between cells and the surface. This advantage of nanotube structure, as described by my proposed model, can be used to calculate the actual adhesion forces using cell detachment technique, which will be described in chapter 10 of this thesis as future work. In fact, the geometry of self-assembled $\mathrm{TiO}_{2}$ nanotubes on the surface of Ti implants can be modeled as a series of cantilevers protruding normal to the implant surface with spring stiffness $K$ subjected to a shear force at the free end. In practice, the cell uses the nanotubes as anchors and then contracts itself to move forward, which can result in bending the nanotubes. In order to quantify the nanotube stiffness variation in terms of the nanotube length, the parameter $K_{a}$ was calculated for various nanotubes obtained under different anodization voltage. It was observed that the stiffness of the nanotubes decreases by the increase in the nanotube length and anodization time.

It is generally accepted that nanotube diameter affects cell proliferation. However, there is lack of data on the effect of nanotube length on cell growth. I showed that not only diameter but also nanotube length affects cell number, the two being expressed as stiffness. The results indicate that nanotubes with the same crystalline structure and chemical composition but higher anodization duration (and consequently higher length and diameter) show a decrease in the number of attached cells. In the case of 
amorphous (as anodized) cp-titania nanotubes, the anodization duration of $240 \mathrm{~min}$ shows the higher number of attached cells $\left(1500\right.$ cells $\left./ \mathrm{mm}^{2}\right)$ in comparison to nanotubes anodized for $960 \mathrm{~min}\left(1100\right.$ cells $\left./ \mathrm{mm}^{2}\right)$. This finding signifies the importance of considering both the diameter and length of nanotubes on cell number and attachment. Therefore, I believe that the main reason for the nanotube geometric effect (diameter and length) on increased osteoblast cell number and attachment is the change in stiffness of the nanotubes. The nanotubes with higher stiffness $\left(\mathrm{k}=14.2 \times 10^{-3} \mathrm{nN} / \mu \mathrm{m}\right)$ promote more cell number (up to 1500 cells $/ \mathrm{mm}^{2}$ ) in comparison to nanotubes with lower stiffness $(\mathrm{k}=$ $\left.8.9 \times 10^{-4} \mathrm{nN} / \mu \mathrm{m}\right)$ and cell count of 1000 cells $/ \mathrm{mm}^{2}$. These observations can be perhaps explained by the fact that mechanical input from the substrate will be transmitted through adhesion receptors, then it may activate downstream signals that regulate both the degree of cell spreading and the rate of growth. On stiff substrates, resistance to mechanical probing may lead to protein conformational changes and activation of signaling enzymes at the adhesion sites. The response in turn causes an increase in traction forces and in cell spreading.

The conclusion was that it is easier for the cells to use the stiffer nanotubes as anchoring steps for their attachment and migration. Mechanical input from the substrate is transmitted through adhesion receptors, and then it activates downstream signals that regulate both the degree of cell spreading and the rate of growth. Since the stiffer nanotubes can promote more mechanical output for the probing cells, perhaps more mechanical input from the nanotubes can be transmitted through adhesion receptors and more signals for cell proliferation will be activated. These phenomena could finally result in higher cell proliferation on stiffer nanotubes. 


\section{Chapter 10}

\section{Future Work}

In this chapter I will outline a number of future directions and extensions to my $\mathrm{PhD}$ research work. The goal of these directions is to overcome a few obstacales in clinical applications of the $\mathrm{TiO}_{2}$-coated biomedical implants. These extensions are (i) the enhancement of cell adhesion via cell adhesion force quantification, (ii) studies of antibacterial disinfection, (iii) understanding drug therapy, (iv) research on cancer therapy with $\mathrm{TiO}_{2}$ nanotubes, and (v) analysis of biocompatibility.

\subsection{Evaluation of Osteoblast Cell Adhesion to $\mathrm{TiO}_{2}$ Nanotube Surfaces}

I showed that nanotubular surface modification of titanium enhances the osteogenic environment on this surface. However, the underlying mechanism remains unknown and its potential application to other tissue engineering materials has not been explored. This research will determine the adhesion forces of a single osteoblast on a $\mathrm{TiO}_{2}$ nanotube thin layer.

For this purpose a cell detachment technique can be used to evaluate the adhesive nature of a single osteoblast on $\mathrm{TiO}_{2}$ nanotube surfaces. This technique is shear based and its principle is similar to the scratch test well established in the field of engineering and material sciences. Rat bone marrow-derived osteoblasts can be cultured on nanotubemodified samples. After an incubation for several hours, the mean critical shear force required to initiate detachment of a single osteoblast can be determined on surface modified $\mathrm{TiO}_{2}$ nanotube samples, and compared with the force required on untreated surfaces. Osteoblasts cultured on $\mathrm{TiO}_{2}$ nanotube surfaces can be characterized and the level and density of vinculin expression, focal contact formation and both total expression and density of actin fibers can be investigated and compared with plain surfaces. This research can determine if each individual cell attached to the $\mathrm{TiO}_{2}$ surfaces is substantially more resistant to exogenous load potentially from blood and fluid flow and mechanical force in the initial stage of the in-vivo biological environment. 


\subsection{Antibacterial Properties of Titania-Nanotube Surfaces}

A major burden for orthopedic implant success is due to chronic infections, which can result in implant failure and revision surgeries. Surface modification of implants with nanofabricated surfaces has shown to reduce bacteria attachment and allow tissue integration. Webster et al. compared conventional bare titanium surfaces with titanium surfaces that were modified by nanoroughing through electron beam evaporation. Their results showed the reduction of $S$. aureus, S. epidermidis, and P.aeruginosa (bacteria that limit orthopedic implant function and efficacy) adhesion in nanorough surfaces compared to conventional surfaces. The same approach can be applied to investigate the antibacterial properties of $\mathrm{TiO}_{2}$ nanotubes. Since $\mathrm{TiO}_{2}$ nanotubes can also be considered a nanoroughned surface this is expected to reduce bacterial adhesion. However, the mechanism behind this selective adhesion is unexplored. The reason behind why nanoroughned surfaces encourage osteoblast adhesion and bone integration, while at the same time discourage bacteria that cause infection to adhere on the surface, is an exciting topic to be investigated. I have shown that $\mathrm{TiO}_{2}$ nanotubes increase osteoblast number. Therefore, investigations of the reduced bacterial and increased osteoblast number by $\mathrm{TiO}_{2}$ nanotubes can work in synergy together for optimized implant modification. As will be discussed below, it is also possible to further improve the anti-infection and proosseointegration properties of nanotube modified implants by incorporating anti-bacterial drugs as well as growth factors inside the nanotubes.

\subsection{Local Drug Therapy by Titania-Nanotube Array Surfaces}

Acute infection or chronic osteomyelitis develops in as many as 5-33\% of implant surgeries despite the use of strict antiseptic operative procedures. ${ }^{167}$ To reduce the chance of the infection, patients are subjected to antibiotic drug therapy upon initial surgery where the antibiotics are systemically delivered intravenously, intramuscularly or topically. Systemic antibiotic and growth factor delivery results in certain drawbacks such as toxicity and limited bioavailability. Further, high doses of drugs are required in order to make it effective at the site of implantation, which can result in undesired 
side effects. Local antibiotic therapy with nanostructured materials can be a preferred way of administering drugs.

The focus of this research can be on the application of $\mathrm{TiO}_{2}$ nanotube array surfaces for local delivery of antibiotics at the site of implantation. Films comprised of $\mathrm{TiO}_{2}$ nanotube arrays can be loaded with different amounts of drugs (e.g. gentamicin). To facilitate gentamicin release from the nanotubes, the surfaces can be immersed in PBS. The release data can be monitored as a function of nanotube length and diameter from the gentamicin loaded nanotubes. The nanotubes' length and diameter can be correlated to the amounts of drugs at different rates to be eluted at the implant site. Moreover, for evaluating the efficacy of eluting drug in preventing bacteria adhesion and subsequent colony and biofilm formation, a significant pathogenesis of biomaterials-related infections, the investigation of bacterial adhesion on drug-loaded nanotubes can be investigated.

\section{4. $\mathrm{TiO}_{2}$ Nanotubes for Cancer Therapy}

Photothermal properties of $\mathrm{TiO}_{2}$ nanotubes, as a potential therapeutic agent for cancer thermotherapy, has been reported to be an affective way to destroy Murine colon

cancer cells. ${ }^{168}$ These tests were conducted in-vitro. The potential of $\mathrm{TiO}_{2}$ nanotubes to destroy other cancer cells can be investigated particularly with in-vivo tests. Therefore, in-vivo animal tests can be conducted to investigate if the $\mathrm{TiO}_{2}$ nanotubes can inhibit tumor growth. Also research can be made toward a better understanding of any possible side effects due to IR-induced photocatalytic reactions.

In addition, Kalbacova et al. ${ }^{166}$ showed the anticancer properties of $\mathrm{TiO}_{2}$ nanotubes. They used isolated tubes administrated to a tumor, followed by a focused UVlight exposure of the tumor. Upon exposure to UV light the tumor cells changed their viable shape and a considerable amount of them were dead. Their findings demonstrated the photo-induced cancer cell killing capability of the $\mathrm{TiO}_{2}$ nanotubes. However, the clinical application of this technique requires a direct access of the light to the $\mathrm{TiO}_{2}$ nanotubes. Therefore potential approaches such as doping the nanotubes can be examined to obtain effects at longer wavelength for higher penetration depth. Moreover further 
in-vivo experiments can be performed to test the efficacy of the UV exposed $\mathrm{TiO}_{2}$ nanotube cancer therapy technique.

\subsection{Biocompatibility of $\mathrm{TiO}_{2}$ Nanotubes}

The biological safety of nanotechnology is a largely untested area. It is not known what would happen if the nanotubes on the implant's surface detach from the implant surface. Will they be filtered and collected in the kidney? Can they damage the brain function?

The goal of this research program will be to investigate the interaction between artificial nanostructured biomaterials and biological systems with emphasis on the mammalian physiological systems. This study can span all aspects from biomolecular adsorption on artificial chemically and/or topographically $\mathrm{TiO}_{2}$ nanostructured materials through the subsequent cell adhesion to tissue regeneration and long term performance of biomaterials in vitro. The ultimate aim is to reproduce and enhance the natural nanoscale elements present in the human body and to thereby develop new materials with improved biological activities. Plans can be made to carry out experiments involving the cytotoxicity assessment using tetrazotium bromide (MTT) assay. Cell membrane impairment will be studied using lactae dehydrogenase (LDH) release assay. 
Appendix 


\section{Appendix 1: Copyright for the Manuscript Published in Applied Physics Letters}

Asthana, A.; Shokuhfar, T.; Gao, Q.; Heiden, P.A.; Friedrich, C.; Yassar, R. S. A Study

on the Modulation of the Electrical Transport by Mechanical Straining of Individual

TiO2 Nanotube, Applied Physics Letters, 2010, 97, 072107-072110

Rightslink@ by Copyright Clearance Center

11/21/10 10:41 PM

\section{Powered by $\mathbf{R} \mathbf{G}$ H T S LIN K $>$ Home $\begin{gathered}\text { Account } \\ \text { Info }\end{gathered}$ Help}

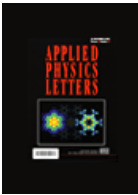

Title:

A study on the modulation of Logged in as: the electrical transport by Tolou Shokuhfar

mechanical straining of olou Shokuhfar

individual titanium dioxide

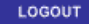

$* 8$

nanotube

Author: $\quad$ A. Asthana, T. Shokuhfar, Q.

Gao, et al

Publication: Applied Physics Letters

Volume/Issue $97 / 7$

Publisher: American Institute of Physics

Date: $\quad$ Aug 17, 2010

Page Count: 3

ID: $\quad$ To be determined

Copyright (๑) 2010, American Institute of Physics

Order Completed

Thank you very much for your order.

This is a License Agreement between Tolou Shokuhfar ("You") and American Institute of Physics ("AIP"). The license consists of your order details, the terms and conditions provided by American Institute of Physics, and the payment terms and conditions.

Get the printable license.

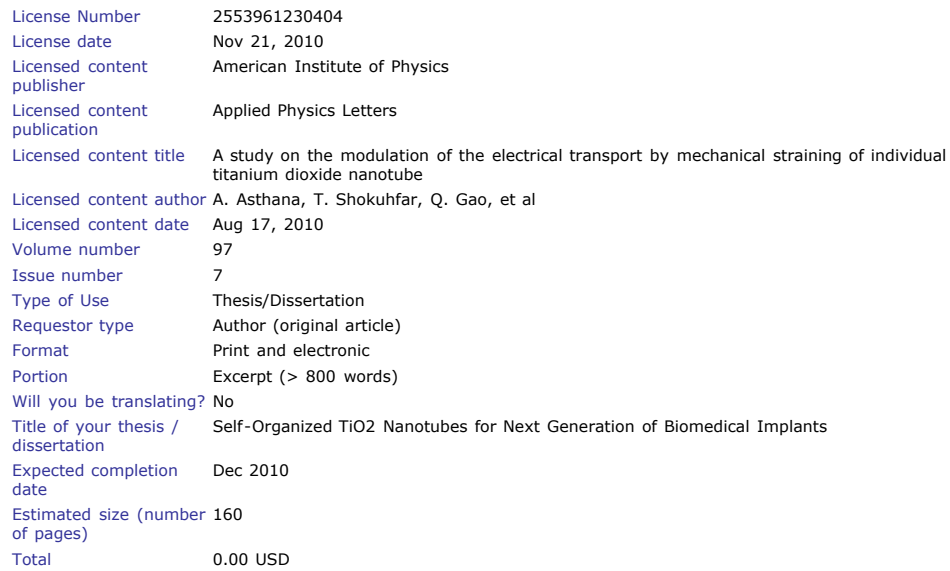




\section{Appendix 2: Copyright for the Manuscript Published in Applied Science Letters}

\section{Asthana, A.; Shokuhfar, T.; Gao, Q.; Heiden, P.A.; Friedrich, C.; Yassar, R.S. A Real Time Observation of Phase Transition of Anatase $\mathrm{TiO}_{2}$ Nanotubes Into Rutile Particles by In-Situ Joule Heating Inside Transmission Electron Microscope, Advanced Science Letters, 2010, 3, 557-562}

RE: Permission

From: nalwa@mindspring.com

To: tshokuhf@mtu.edu

Dear Tolou Shokuhfar:

American Scientific Publishers grants permission to reuse your entire article published in Advanced Science Letters in your $\mathrm{PhD}$ thesis (print + online editions) to be published by the

Michigan Technological University.

"A Real Time Observation of Phase Transition of Anatase TiO2 Nanotubes Into Rutile Nanoparticles by InSitu Joule Heating Inside Transmission Electron Microscope" by Asthana, A.; Shokuhfar, T.; Gao, Q.;

Heiden, P.A.; Friedrich, C.; Yassar, R.S.

Source: Advanced Science Letters, Volume 3, Number 4, December 2010, pp. 557-562.

H. S. Nalwa

American Scientific Publishers

www.aspbs.com/science

-----Original Message-----

From: Tolou Shokuhfar [mailto:tshokuhf@mtu.edu]

Sent: Sunday, November 21, 2010 7:53 PM

To: science@aspbs.com

Subject: Permission

Dear Prof. Nalwa,

I am writing this email to obtain your permission to reuse an article that is published in Advanced Science Letters. I am a co-author for this article and I would like to reuse this in my PhD thesis.

Below is the article information:

"A Real Time Observation of Phase Transition of Anatase TiO2 Nanotubes Into Rutile Nanoparticles by InSitu Joule Heating Inside Transmission Electron Microscope"

Authors: Asthana, A.; Shokuhfar, T.; Gao, Q.; Heiden, P.A.; Friedrich, C.; Yassar, R.S

Source: Advanced Science Letters, Volume 3, Number 4, December 2010 , pp. 557-562(6)

The title of my thesis is "Self-Orderd TiO2 Nanotubes for Next Generation Biomedical Implants" and will be published in Dec 2010.

Please let me know if you need any further information regarding this request. I appreciate your attention,

Regards, 


\section{Appendix 3: Copyright for the Manuscript Published in Journal of Applied Physics}

Shokuhfar, T.; Gao, Q.; Asthana, A.; Walzack, K.; Heiden, P.A.; Friedrich, C. Structural

Instabilities in $\mathrm{TiO}_{2}$ Nanotubes, Journal of Applied Physics. 2010, 108, 104310

Rightslink@ by Copyright Clearance Center

$11 / 22 / 10$ 11:20 PM

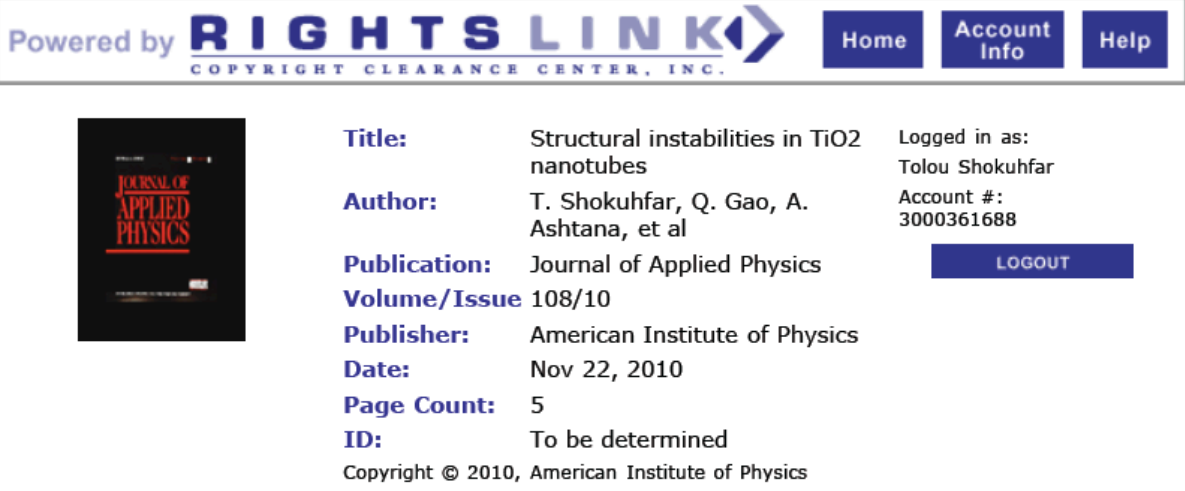

Order Completed

Thank you very much for your order.

This is a License Agreement between Tolou Shokuhfar ("You") and American Institute of Physics

("AIP"). The license consists of your order details, the terms and conditions provided by American

Institute of Physics, and the payment terms and conditions.

\section{Get the printable license.}

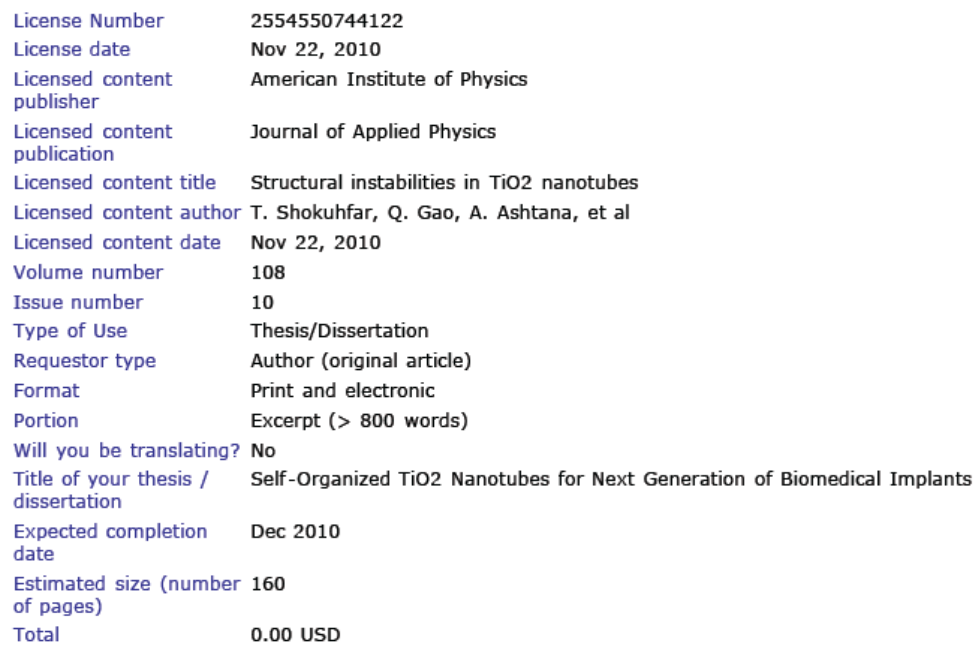




\title{
Appendix 4: Copyright for the Manuscript Published in ACS Nano
}

\author{
Shokuhfar, T.; Arumugam, G. K.; Heiden P. A.; Yassar, R. S.; Friedrich, C. Direct \\ Compressive Measurements of Individual Titanium Dioxide Nanotubes, ACS Nano, \\ 2009, 3, 3098.
}

\section{American Chemical Society's Policy on Theses and Dissertations}

If your university requires a signed copy of this letter see contact information below.

Thank you for your request for permission to include your paper(s) or portions of text from your paper(s) in your thesis. Permission is now automatically granted; please pay special attention to the implications paragraph below. The Copyright Subcommittee of the Joint Board/Council Committees on Publications approved the following:

Copyright permission for published and submitted material from theses and dissertations

ACS extends blanket permission to students to include in their theses and dissertations their own articles, or portions thereof, that have been published in ACS journals or submitted to ACS journals for publication, provided that the ACS copyright credit line is noted on the appropriate page(s).

Publishing implications of electronic publication of theses and dissertation material Students and their mentors should be aware that posting of theses and dissertation material on the Web prior to submission of material from that thesis or dissertation to an ACS journal may affect publication in that journal. Whether Web posting is considered prior publication may be evaluated on a case-by-case basis by the journal's editor. If an ACS journal editor considers Web posting to be "prior publication", the paper will not be accepted for publication in that journal. If you intend to submit your unpublished paper to ACS for publication, check with the appropriate editor prior to posting your manuscript electronically.

If your paper has not yet been published by ACS, we have no objection to your including the text or portions of the text in your thesis/dissertation in print and microfilm formats; please note, however, that electronic distribution or Web posting of the unpublished paper as part of your thesis in electronic formats might jeopardize publication of your paper by ACS. Please print the following credit line on the first page of your article: "Reproduced (or 'Reproduced in part') with permission from [JOURNAL NAME], in press (or 'submitted for publication'). Unpublished work copyright [CURRENT YEAR] American Chemical Society." Include appropriate information.

If your paper has already been published by ACS and you want to include the text or portions of the text in your thesis/dissertation in print or microfilm formats, please print the ACS copyright credit line on the first page of your article: "Reproduced (or 'Reproduced in part') with permission from [FULL REFERENCE CITATION.] Copyright [YEAR] American Chemical Society." Include appropriate information.

Submission to a Dissertation Distributor: If you plan to submit your thesis to UMI or to another dissertation distributor, you should not include the unpublished ACS paper in your thesis if the thesis will be disseminated electronically, until ACS has published your paper. After publication of the paper by ACS, you may release the entire thesis (not the individual ACS article by itself) for electronic dissemination through the distributor; ACS's copyright credit line should be printed on the first page of the ACS paper.

Use on an Intranet: The inclusion of your ACS unpublished or published manuscript is permitted in your thesis in print and microfilm formats. If ACS has published your paper you may include the manuscript in your thesis on an intranet that is not publicly available. Your ACS article cannot be posted electronically on a publicly available medium (i.e. one that is not password protected), such as but not limited to, electronic archives, Internet, library server, etc. The only material from your paper that can be posted on a public electronic medium is the article abstract, figures, and tables, and you may link to the article's DOI or post the article's author-directed URL link provided by ACS. This paragraph does not pertain to the dissertation distributor paragraph above.

Questions? Call +1 202/872-4368/4367. Send e-mail to copyright@ acs.org or fax to +1 202-776-8112. 10/10/03, 01/15/04, 06/07/06 


\section{References}

1 Zhua, X.; Chena, J.; Scheidelera, L.; Reichlb, R.; Geis-Gerstorfer, J. Effects of Topography and Composition of Titanium Surface Oxides on Osteoblast Responses. Biomaterials, 2004, 25, 4087-4103.

2 Ercan, B.; Webster, T.J. The Effect of Biphasic Electrical Stimulation on Osteoblast Function at Anodized Nanotubular Titanium Surfaces, Biomaterials, 2010, 31, 36843693.

${ }^{3}$ Park, J.; Bauer, S.; Schlegel, K.A.; Neukam, F.W.; Mark, K.V.D.; Schmuki, P. TiO2 Nanotube Surfaces: $15 \mathrm{~nm}$ - an Optimal Length Scale of Surface Topography for Cell Adhesion and Differentiation. Small 2009, 5, 666-71.

${ }^{4}$ Chongmu, L.; Chanseok, H.; Hohyeong, K.; Jungwoo, K.; Hong, M. Z. TiO2 Nanotubes as a Therapeutic Agent for Cancer Thermotherapy, Photochem. Photobiol., 2010, 86, 981-989.

${ }^{5}$ Martin, J. Y.; Schwartz, Z. ; Hummert, T. W. ; Schraub, D. M. ; Simpson, J. ; Lankford Jr. J.; Dean, D. D. ; Cochran, D. L. ; Boyan, B. D. Effect of Titanium Surface Roughness on Proliferation, Differentiation, and Protein Synthesis of Human Osteoblast-like Cells (MG63), J. Biomed. Mater. Res. 2004, 29, 389-401.

${ }^{6}$ Kima, S.K.; Lima, J. H.; Lee, S.Ch.; Namb, S.Ch.; Kangc, H.G; Choi, J. Anodically Nanostructured Titanium Oxides for Implant Applications, Electrochimica Acta. 2008, $53,4846-4851$.

${ }^{7}$ Branemark, P-I.; Adell, R.; Breine, U.; Hansson, BO.; Lindstrom, J.; Ohlsson, A. IntraOsseous Anchorage of Dental Prostheses. I.Experimental Studies. Scand. J. Plast. Reconstr. Surg. 1969, 3, 81-100.

8 Adell, R.; Lekholm, U.; Rockler, B.; Branemark, PI. A 15-year Study of Osseointegrated Implants in the Treatment of the Edentulous Jaw. Int. J. Oral Surg. 1981,10, 387-416.

${ }^{9}$ Wang, D.; Christensen, K.; Chawla, K. ; Xiao, G. ; Krebsbach, P. H. ; Francesch, R. T. Isolation and Characterization of MC3T3-E1 Preosteoblast Subclones with Distict In Vitro and In Vivo Differentiation/Mineralization Potential. J. Bone Miner. Res., 1999, 14 893-903. 
${ }^{10}$ Wilke, H-J.; Claes, L.; Steinemann S. The Influence of Various Titanium Surfaces on the Interface Shear Strength Between Implants and Bone. Adv. Biomater. Clin. Impl. Mater. 1990, 14, 309-314.

${ }^{11}$ Gotfredsen K, Wennerberg A, Johansson C, Skovgaard LT, Hjorting-Hansen E. Anchorage of TiO2-blasted, HA-Coated and Machined Implants: An experimental study with rabbits. J. Biomed. Mater. Res. 1995, 29,1223-1231.

${ }^{12}$ Buser, D.; Schenk, R.K.; Steinemann, S.; Fiorellini, J.P.; Fox, C.H.; Stich, H. Influence of Surface Characteristics on Bone Integration of Titanium Implants: A Histomorphometric Study in Miniature Pigs. J. Biomed. Mater. Res. 1991, 25, 889-902.

${ }^{13}$ Kieswetter, K.; Schwartz, Z.; Hummert, T.W.; Cochran, D.L.; Simpson, J.; Dean, D.D.; Boyan, B.D; Surface Roughness Modulates the Local Production of Growth Factors and Cytokines by Osteoblastlike MG-63 Cells. J. Biomed. Mater. Res. 1996, 32, 55-63.

${ }^{14}$ Boyan, B.D.; Batzer, R.; Kieswetter, K.; Liu, Y.; Cochran, D.L.; Szmuckler-Moncler, S.; Dean, D.D.; Schwartz, Z. Titanium Surface Roughness Alters Responsiveness of MG63 Osteoblast-Like Cells to 1 Alpha, 25-(OH)2D3. J. Biomed. Mater. Res. 1998, 39, $77-85$.

${ }^{15}$ Cochran, D.L.; Schenk, R.K.; Lussi, A.; Higginbottom F.L.; Buser D. Bone Response to Unloaded and Loaded Titanium Implants with a Sandblasted and Acid-Etched Surface: A Histometric Study in the Canine Mandible. J. Biomed. Mater. Res. 1998, 40, 1-11.

${ }^{16}$ Cochran, D.L.; Hermann ,J.S.; Schenk, R.K.; Higginbottom F.L.; Buser, D. Biological Width Around Titanium Implants: A Histometric Analysis of the Implanto-Gingival Junction Around Unloaded and Loaded Nonsubmerged Implants in the Canine Mandible. J. Periodontol. 1997, 68:186-198.

${ }^{17}$ Cochran, D.L. The Scientific Basis for and Clinical Experiences with Staumann Implants Including the ITI Dental Implant System: A Consensus Report. Clin. Oral Impl. Res. 2000, 11, 33-58.

${ }^{18}$ Cochran, D.L.; Buser, D.; Bruggenkate, C.M.; Weingart, D.; Taylor, T.M.; Bernard, J.P.; Peters, F.; Simpson, JP. The Use of Reduced Healing Times on ITI Implants with a Sandblasted and Acidetched (SLA) Surface: Early Results from Clinical Trials on ITI SLA implants. Clin. Oral Impl. Res. 2002, 13,144-53.

${ }^{19}$ Roccuzzo, M.; Bunino, M.; Prioglio, F.; Bianchi, S.D. Early Loading of Sandblasted and Acid-Etched (SLA) Implants: A Prospective Split-Mouth Comparative Study-OneYear Results. Clin Oral Impl. Res. 2001, 12, 572-578. 
${ }^{20}$ Li, D.; Ferguson S.; Beutler, T.; Cochran, D. L.; Sittig, C.; Hirt, H. P.; Buser, D. Biomechanical Comparison of the Sandblasted and Acid-Etched and the Machined and Acid-Etched Titanium Surface for Dental Implants, J. Biomed. Mater. Res. 2002, 60, 325-332.

${ }^{21}$ Kim, S.E.; Lim, J. H.; Lee , S. Ch.; Nam, S.Ch.; Kang, H.G.; Choi , J. Anodically Nanostructured Titanium Oxides for Implant Applications, Electrochimica Acta. 2008, $53,4846-4851$.

22 Wennerberg, A.; Hallgren, C.; Johansson, C.; Danelli, S. A Histomorphometric Evaluation of Screw-Shaped Implants Each Prepared with Two Surfaces Roughnesses, Clin. Oral Implants Res. 1998, 9, 11-19.

${ }^{23}$ Juodzbalys, G.; Sapragoniene, M.; Wennerberg,V.; New Acid Etched Titanium Dental Implant Surface, Stomatologija, Baltic Dental and Maxillofacial J., 2003, 5, 101-105.

${ }^{24}$ Yuan, Z.Y.; Su, B.L.; Titanium Oxide Nanotubes, Nanofibers and Nanowires, Coll. Surf. A 2004, 241, 173.

${ }^{25}$ Gong, D.; Grimes C. A.; Oomman K. V.; Wenchong H.; Singh R.S.; Chen Z.; Dickey E.C.; Titanium Oxide Nanotube Arrays Prepared by Anodic Oxidation, J. Mater. Res. 2001, 16, 3331 .

${ }^{26}$ Hamasaki, N.; Suzuki, M.; Kimura, M; Shirai, H; Hanabusa, K., Preparation of Helical Transition-Metal Oxide Tubes Using Organogelators as Structure-Directing Agents, $J$. Am. Chem. Soc. 2002, 124, 6550-6558.

27 Guo, Y.G.; Hu, J.S.; Liang, H.P.; Wan, L.J.; Bai, C.L. TiO 2 -Based Composite Nanotube Arrays Prepared Via Layer-By-Layer Assembly. Adv. Funct. Mater. 2005, 15, 196-201.

${ }^{28}$ Kasuga, T.; Hiramatsu, M.; Hoson, A.; Sekino, T.; Niihara, K. Formation of Titanium Oxide Nanotube, Langmuir, 1998, 14, 316-403.

${ }^{29}$ Choi, J.; Wehrspohn, R.B.; Lee, J.; Gosele, U. Anodization of Nanoimprinted Titanium: A Comparison With Formation of Porous Alumina, Electrochim. Acta, 2004, 49, 2645.

${ }^{30}$ Chu, R.; Yan, J.; Lian, S.; Wang, Y.; Yan, F.; Chen, D., Shape-Controlled Synthesis of Nanocrystalline Titania at Low Temperature, Solid State Commun. 2004, 130, 789-795.

${ }^{31}$ Zhao, J.; Wang, X.; Chen, R.; Li, L. Fabrication of Titanium Oxide Nanotube Arrays by Anodic Oxidation, Solid State Commin. 2005, 134, 705-719. 
32 Komarneni, S.; Rajha, T. K.; Katsuki, H. Microwave-Hydrothermal Processing of Titanium Dioxide, Mater. Chem. Phys. 1999, 61, 503.

${ }^{33}$ Gong, D.; Grimes, C. A.; Oomman, K.; Varghese, G.R.; Wenchong, H.; Singh, R.S.; Chen, Z.; Dickey, E.C. Titanium Oxide Nanotube Arrays Prepared by Anodic Oxidation, J. Mater. Res. 2001, 16, 3331-3339.

${ }^{34}$ Cai, Q.Y.; Paulose, M.; Varghese, O.K.; Grimes, C.A.; The Effect of Electrolyte Composition On The Fabrication Of Self-Organized Titanium Oxide Nanotube Arrays By Anodic Oxidation, J. Mater. Res. 2005, 20, 230-238.

${ }^{35}$ Xiao, X.F.; Ouyang, K.G.; Liu, R.F. Anatase Type Titania Nanotube Arrays Direct Fabricated by Anodization Without Annealing. Appl. Surf. Sci. 2009, 255, 3659-3667.

${ }^{36}$ Lai, Y.K.; Sun, L.; Chen, C., Nie, C.G.; Zuo, J.; Lin, C.J. Optical and electrical Characterization of $\mathrm{TiO}_{2}$ Nanotube Arrays on Titanium Substrate, Appl. Surf. Sci. 2005, 252, 1101-1108.

${ }^{37}$ Paulose, M.; Grimes, C. A. Anodic Growth of Highly Ordered $\mathrm{TiO}_{2}$ Nanotube Arrays to $134 \mu \mathrm{m}$ in Length, J. Phys. Chem. Lett. 2006, 110, 16179-16182.

38 Yuan, Z.Y.; Su, B.L. Titanium Oxide Nanotubes, Nanofibers and Nanowires, Coll. Surf. A, 2004, 241, 173-179.

${ }^{39}$ Wang, D.; Liu, W. Synthesis and Characterization of Anatase $\mathrm{TiO}_{2}$ Nanotubes With Uniform Diameter From Titanium Powder, Mater. Lett. 2008, 62, 1819-1832.

40 Suzukia, Y.; Yoshikawa, S. Synthesis and Thermal Analyses of $\mathrm{TiO}_{2}$-Derived Nanotubes Prepared by The Hydrothermal Method, J. Mater. Res. 2004, 19, 982-988.

${ }^{41}$ Zhang, M.; Bando, Y.; Wada, K. Sol-Gel Template Preparation of $\mathrm{TiO}_{2}$ Nanotubes and Nanorods, J. Mater. Sci. Lett. 2001, 20, 167-172.

${ }^{42}$ Qiu, J.J.; Yu, W.D.; Gao, X.D.; Li, X.M. Fabrication and Characterization of $\mathrm{TiO}_{2}$ Nanotube Arrays Having Nanopores in Their Walls by Double-Template-Assisted SolGel, Nanotechnology 2006, 17, 4695-4699.

${ }^{43}$ Guo, Y.G.; Hu, J.S.; Liang, H.P.; Wan, L.J.; Bai C.L. TiO 2 -Based Composite Nanotube Arrays Prepared via Layer-by-Layer Assembly, Adv. Funct. Mater. 2005, 15, 196-201.

${ }^{44}$ Paulose, M.; Shankar, K.; Yoriya, S.; Prakasam, H.E.; Varghese, O.K.; Moor, G.K. Anodic Growth of Highly Ordered TiO2 Nanotube Arrays to $134 \mu \mathrm{m}$ in Length, J. Phys. Chem. B, 2006, 110, 16179. 
${ }^{45}$ Zhao J.; Wang X.; Chen R.; Li L. Fabrication of Titanium Oxide Nanotube Arrays by Anodic Oxidation, Solid State. Comm. 2005, 134, 705-710.

46 Prakasam H.E.; Shankar K.; Paulose M.; Varghese O.K.; Grimes C.A.; A New Benchmark for TiO2 Nanotube Array Growth by Anodization, J. Phys. Chem. C 2007, $111,7235-7242$.

${ }^{47}$ Mor, G. K.; Varghese, O. K.; Paulose, M.; Grimes, C. A. Fabrication of Tapered, Conical-Shaped Titania Nanotubes. J. Mater. Res. 2003, 18, 2588-2593.

${ }^{48}$ Varghese, O. K.; Gong, D.; Paulose, M.; Ong, K. G.; Grimes, C. A. Hydrogen Sensing Using Titania Nanotubes. Sens. Actuators B 2003, 93, 338-344.

${ }^{49}$ Varghese, O. K.; Gong, D.; Paulose, M.; Ong, K. G.; Dickey, E. C.; Grimes, C. A. Extreme Changes in the Electrical Resistance of Titania Nanotubes with Hydrogen Exposure. Adv. Mater. 2003, 15, 624-627.

${ }^{50}$ Mor, G. K.; Shankar, K.; Paulose, M.; Varghese, O. K.; Grimes, C. A. Enhanced Photocleavage of Water Using Titania Nanotube Arrays. Nano Lett. 2005, 5, 191-195.

${ }^{51}$ Gong, D.; Grimes, C.A.; Varghese, O.K.; Hu, W.; Singh, R.S.; Chen, Z.; Dickey, E.C.; Titanium Oxide Nanotube Arrays Prepared by Anodic Oxidation. J. Mater. Res. 2001, 16, 3331-3334.

52 Paulose, M.; Mor, G. K.; Varghese, O. K.; Shankar, K.; Grimes, C. A. Visible Light Photoelectrochemical and Water-Photoelectrolysis Properties of Titania Nanotube Arrays. J. Photochem. Photobiol. 2006, 178, 8-15.

${ }^{53}$ Varghese, O. K.; Paulose, M.; Shankar, K.; Mor, G. K.; Grimes, C. A. Water-Photolysis Properties of Micron-Length Highly-Ordered Titania Nanotube-Arrays. J. Nanosci. Nanotech. 2005, 5, 1158-1165.

${ }^{54}$ Albu, S. P.; Ghicov, A.; Macak, J. M.; Hahn, R.; Schmuki, P. Self-Organized, FreeStanding $\mathrm{TiO}_{2}$ Nanotube Membrane for Flow-Through Photocatalytic Applications. Nano Lett. 2007, 7, 1286-1289.

${ }^{55}$ Parkhutik, V. P.; Shershulsky, V. I. Theoretical Modelling of Porous Oxide Growth on Aluminium. J. Phys. D: Appl. Phys. 1992, 25, 1258-1263.

${ }^{56}$ Shobha, T.; Sarma, C. S. N.; Sastry, K. S.; Anjaneyulu, Anodization of hafnium in phosphate baths: Radio tracer studies. C. Bull. Electrochem. 2001, 17, 519-523. 
${ }^{57}$ Lu, Q.; Hashimoto, T.; Skeldon, P.; Thompson, G. E.; Habazaki, H.; Shimizu, K. Nanoporous Anodic Niobium Oxide Formed in Phosphate/Glycerol Electrolyte. Electrochem. Solid-State Lett. 2005, 8, B17-B19.

${ }^{58}$ Mor, G. K.; Varghese, O. K.; Paulose, M.; Shankar, K.; Grimes, C. A. A Review on Highly Ordered, Vertically Oriented $\mathrm{TiO}_{2}$ Nanotube Arrays: Fabrication, Material Properties, and Solar Energy Applications. Sol. Energy Mater. Sol. Cells. 2006, 90, 2011 2075.

${ }^{59}$ Crawford, G.A.; Chawla, A, N.; Das, K.; Bose, S.; Bandyopadhyay , A. Microstructure and Deformation Behavior of Bioactive $\mathrm{TiO}_{2}$ Coatings. Acta Biomat. 2007, 3, 359-367.

${ }^{60}$ Golberg, D.; Costa, P. M. F. J.; Lourie, O; Mitome, M.; Bai, X.; Kurashima, K.; Zhi, C.; Tang, C.; Bando,Y. Direct Force Measurements and Kinking under Elastic Deformation of Individual Multiwalled Boron Nitride Nanotubes. Nano Lett., 2007, 7, 2146-2151.

${ }^{61}$ Ertsa, D.; Lohmusb, A.; Lohmusb, R.; Olinc, H.; Pokropivnyd, A.V.; Ryenc, L.; Svensson, K. Force Interactions and Adhesion of Gold Contacts Using a Combined Atomic Force Microscope and Transmission Electron Microscope, Appl. Surf. Sci. 2002, $188,460-466$.

${ }^{62}$ Yakobson, B.I.; Campbell, M.P.; Brabec, C.J.; Bernholc, J., High Strain Rate Fracture and C-Chain Unraveling in Carbon Nanotubes. Comp. Mater. Sci. 1997, 8, 341-348.

${ }^{63}$ Asahi, R.; Morikawa, T.; Ohwaki, T.; Aoki, K.; Taga, Y. Visible-Light Photocatalysis in Nitrogen-Doped Titanium Oxides, Science 2001, 293-269.

64 Xu, A.; Zhu, J.; Gao, Y.; Liu, H. Iron-Doped Titania Nanoparticles for The Photocatalytic Oxidative Degradation of Nitrite, Chem. Res. Chin. Univ. 2001, 17, 281289.

${ }^{65}$ Bai, X.; Goldberg, D.; Bando, Y.; Zhi, C.; Tang, C.; Mitome, M.; Kurashima, K. Deformation-Driven Electrical Transport of Individual Boron Nitride Nanotubes, Nano Lett 2007,7, 632-639.

${ }^{66}$ Zhang, S.; Chen, Q.; Peng, L. M. Structure and formation of $\mathrm{H}_{2} \mathrm{Ti}_{3} \mathrm{O}_{7}$ nanotubes in an alkali environment, Phys. Rev. B, 2005, 71, 014104-014109.

${ }^{67}$ O’Regan, B.; Graetzel, M. A Low-Cost, High-efficiency Solar Cell Based on DyeSensitized Colloidal $\mathrm{TiO}_{2}$ Films, Nature, 1991, 353, 737-743.

${ }^{68}$ Shokuhfar, T.; Arumugam, G. K.; Heiden P. A.; Yassar, R. S.; Friedrich, C. Direct Compressive Measurements of Individual Titanium Dioxide Nanotubes, ACS Nano, 2009, 3, 3098 . 
69 Zhang, Z. Y.; Jin, C. H.; Liang, X. L.; Chen, Q.; Peng, L.-M.; Current-Voltage Characteristics and Parameter Retrieval of Semiconducting Nanowires, Appl. Phys. Lett. 2006, 88, 0731021-0731029.

${ }^{70}$ Peng, H. Y.; McCluskey, M. D.; Gupta, Y. M.; Kneissl, M. A. ; Johnson, N. M. ShockInduced Band-Gap Shift in GaN: Anisotropy of the Deformation Potentials, Physical Review B. 2005, 71, 1152071-1152078.

71 Mahanti, S.D.; Hoang K, Ahmad, Deep Defect States in Narrow Band-Gap Semiconductors, S. Physica B: Condensed Matter. 2007, 401, 291-300.

${ }^{72}$ Robertson, J.; Sharia, O.; Demkov, A. A., Fermi Level Pinning by Defects in HfOMetal Gate Stacks, Appl. Phys. Lett. 2007, 91, 132912-132916.

${ }^{73}$ Huang, M.; Rugheimer, P.; Lagally, M. G.; Liu, F., Bending of Nanoscale Ultrathin Substrates by Growth of Strained Thin Films and Islands, Phys. Rev. B 2005, 72, 085450085459.

${ }^{74}$ Lin, X.; He, X. B.; Yang, T. Z.; Gao, W.; Shi, D. X.; Gao, H. -J, Ma, D. D. D.; Lee, S. T.; Liu,F.; Xie, X. C.; Intrinsic Current-voltage Properties of Nanowires with Four-Probe Scanning Tunneling Microscopy: A Conductance Transition of $\mathrm{ZnO}$ Nanowire, Appl. Phys. Lett. 2006, 89, 043103-043109.

75 Beckenridge, R. G.; Hosler, W. R.; Electrical Properties of Titanium Dioxide Semiconductors, Phys. Rev. 1953, 91, 793-799.

${ }^{76}$ Shokuhfar, T.; Arumugam, G. K.; Heiden, P.A.; Yassar, R. S., Friedrich, C.; Direct Compressive Measurements of Individual Titanium Dioxide Nanotubes, ACS Nano, 2009, 3, 3098-3108.

77 Zwilling, V.; Aucouturier, M.; Darque-Ceretti, E. Anodic oxidation of titanium and TA6V alloy in chromic media. An electrochemical approach, Electrochim. Acta, 1999, 45, 921-928.

${ }^{78}$ Mowbray, D. J. ; Martinez, J. I. ; Lastra J. M.; Thygesen K. S.; Jacobsen, K. W. Stability and Electronic Properties of $\mathrm{TiO}_{2}$ Nanostructures With and Without $\mathrm{B}$ and N Doping, J. Phys. Chem. C. 2009, 113, 12301-12308.

${ }^{79}$ Huang B. S.; Tseng H-H.; Wey, M.-Y. Comparison of Visible-Light-Driven Routes of Anion-Doped $\mathrm{TiO}_{2}$ and Composite Photocatalyst, J. Cer. Soc. Japan, 2009, 117, 753-758.

${ }^{80}$ Asahi R.; Morikawa, T.; Ohwaki, T.; Aoki, K.; Taga, Y. Visible-Light Photocatalysis in Nitrogen-Doped Titanium Oxides, Science, 2001, 293, 269-273. 
${ }^{81}$ Kosowska B.; Mozia, S.; Morawski, A.; Grznil, B.; Janus, M. ; Kalucki, K. The Preparation of $\mathrm{TiO}_{2}$-Nitrogen Doped by Calcination of $\mathrm{TiO}_{2}$ Center Dot xH(2) O Under Ammonia Atmosphere for Visible Light Photocatalysis, Sol. Energy Mater. Cells, 2005, 88269.

${ }^{82}$ Varghese, O. K.; Gong, D.; Paulose, M.; Grimes, C. A.; Dickey, E. C. Crystallization and High-Temperature Structural Stability of Titanium Oxide Nanotube Arrays, J. Mater. Res., 2003, 18, 156- 165.

${ }^{83}$ Bersani, D.; Antonioli, G.; Lottici, P.P.; Lopez, T. Raman Study of Nanosized Titania Prepared by Sol-gel Route, J. Non-Cryst. Solids 1998, 234, 175-181.

84 Sreekantan, S.; Hazan, R.; Lockman, Z. Photoactivity of Anatase-Rutile $\mathrm{TiO}_{2}$ Nanotubes Formed by Anodization Method, Thin Solid Films, 2009, 518, 16-21.

${ }^{85}$ Li, G.; Liu, Z.-Q. ; Lu, J. ; Wang, L. ; Zhang, Z. Effect of Calcination Temperature on the Morphology and Surface Properties of $\mathrm{TiO}_{2}$ Nanotube Arrays, Appl. Surface Sci. 2009, 255, 7323-7328.

86 Dong, L.; Ma, Y.; Wang, Y.; Tian, Y.; Ye, G.; X. Jia, Cao, G. Preparation and Characterization of Nitrogen-Doped Titania Nanotubes, Mater. Lett. 2009, 63,1598-1600.

${ }^{87}$ Varghese, O. K.; Gong, D.; Paulose, M.; Grimes, C. A.; Dickey, E. C. Crystallization and High-Temperature Structural Stability of Titanium Oxide Nanotube Arrays, J. Mater. Res., 2003, 18, 156- 165.

${ }^{88}$ Yang, Y.; Wang, X.; Li, L. Crystallization and Phase Transition of Titanium Oxide Nanotube Arrays, J. Am. Ceram. Soc., 2008, 91, 632-635.

${ }^{89}$ Zheng, R.Y.; Lin, L.; Xie, J.J.; Zhu, Y.X.; Xie, Y.C. State of Doped Phosphorus and Its Influence On The Physicochemical and Photocatalytic Properties of P-Doped Titania, $J$. Phys. Chem. C, 2008, 112, 15502-9.

90 Tsuchiya, T.; Hirata, M.; Chiba, N. Young's Modulus, Fracture Strain, and Tensile Strength of Sputtered Titanium Thin Films, Thin Solid Films, 2005, 484, 245-250.

91 Ting, C. C.; Chen, S. Y.; Liu, D.M. Structural Evolution and Optical Properties of TiO Thin Films Prepared by Thermal Oxidation of Sputtered Ti Films, J. Appl. Phys. 2000, $88,4628-4633$.

92 Rodriguez, R.; Vargas, S.; Arroya, R.; Montiel, R.; Haro, E. Modification of The Phase Transition Temperatures in Titania Doped with Various Cations, J. Mater. Res. 1997, 12, 439-444. 
93 Bokhimi, X.; Novaro, O.; Gonzalez, R. D.; Lopez, T.; Chimal, O.; Asomoza, A.; Gomez, R.; Copper Precursor Effect on Reducibility and Titania Phases Concentration of Sol-Gel Cu/TiO2Catalyst, J. Solid State Chem. 1999, 144, 349-355.

${ }^{94}$ Xia, B.; Huang, H.; Xie, Y. Heat Treatment on TiO2 Nanoparticles Prepared by Vaporphase Hydrolysis., Mater. Sci. Eng. B, 1999, 57, 150-159.

95 Zhang, H.; Banfield, J. F.; Thermodynamic Analysis of Phase Stability of Nanocrystalline Titania, J. Mater. Chem. 1998, 8, 2073-2077.

${ }^{96}$ Zhang, H.; Banfield, J.F. Understanding Polymorphic Phase Transformation Behavior During Growth of Nanocrystalline Aggregates: Insights From TiO2, J. Phys. Chem. B, 2000, 104, 3481-3487.

${ }^{97}$ Kumar, K. N. P.; Engell, J.; Kumar, J.; Keizer, K.; Okubo, T.; Sadakata, M. PoreStructure Stabilization by Controlling Particle Coordination, J. Mater. Sci. Lett. 1995, 14, 1784-1788.

${ }^{98}$ Kumar, K-N.P.; Keizer, K.; Burggraaf, A.J.; Okubo, T.; Nagamoto, H.; Morroka, S.; Densification of Nanostructured Titania Assisted by a Phase Transformation, Nature, 1992, 358,48-51.

99 Shokuhfar, T.; Arumugam, G. K.; Heiden P. A.; Yassar, R. S.; Friedrich, C. Direct Compressive Measurements of Individual Titanium Dioxide Nanotubes, ACS Nano, 2009, 3, 3098-4002.

${ }^{100}$ Balaur, E.; Macak, J.M.; Taveira, L.; Schmuki, P.; 7, Tailoring the wettability of $\mathrm{TiO}_{2}$ nanotube layers, Electrochem. Commun., 2005, 20, 1066-1070.

${ }^{101}$ Macak, J.M.; Hildebrand, H.; Marten-Jahns, U.; Schmuki, P. Mechanistic Aspects and Growth of Large Diameter Self-Organized $\mathrm{TiO}_{2}$ Nanotubes, J. Electroanal. Chem. 2008, 621, 254-266.

102 Balaur, E.; Macak, J.M.; Tsuchiya, H.; Schmuki P. Wetting Behavior of Layers of $\mathrm{TiO}_{2}$ Nanotubes with Different Diameters, J. Mater. Chem. 2005,15, 4488-4491.

${ }^{103}$ Chiang, C.Y.; Chiou, S.H.; Yang, W.E.; Hsu, M.L.; Yung, M.C.; Tsai, M.L.; Chen, L.K.; Huang, H.H; Formation of $\mathrm{TiO}_{2}$ Nano-Network on Titanium Surface Increases the Human Cell Growth, Dent. Mater. 2009, 25, 1022-1029.

${ }^{104}$ Dawson, E.; Mapili, G.; Erickson, K.; Taqvi, S.; Roy, K.; Biomaterials for Stem Cell Differentiation, Adv. Drug Deliv. Rev. 2008, 60, 215-228. 
105 Ninham, B.W.; Kurihara, K.; Vinogradova, O.I. Hydrophobicity, Specific Ion Adsorption and Reactivity, Colloid. surf. A, 1997, 123, 7-12.

${ }^{106}$ Nosonovsky, M.; Bhushan, B. Superhydrophobic Surfaces and Emerging Applications: Non-Adhesion, Energy, Green Engineering, Curr. Opin. colloid Interface Sci., 2009, 14, 270-280.

${ }^{107}$ Hazen, K.C.; Brawner, D.L.; Riesselman, M.H., Jutila, M.A. Differential Adherence of Hydrophobic and Hydrophilic Candida albicans Yeast Cells to Mouse Tissues Infect. Immun. 1991, 59, 907-912.

108 Kwon, Y.; Patankar, N.; Choi J.; Lee, J.; Design of Surface Hierarchy for Extreme Hydrophobicity, Langmuir, 2009, 25, 6129-6136.

${ }^{109} \mathrm{Hu}, \mathrm{H}$; Larson, G. R. Evaporation of a Sessile Droplet on a Substrate, J. Phys. Chem. B 2002,106, 1334-1344.

${ }^{110}$ Hartland, S.; Hartley, R.W. Axisymmetric Fluid-Liquid Interfaces, Elsevier Publisher, Amsterdam, New York, 1976.

111 Ghicov, A.; Schmuki, P. Self-Ordering Electrochemistry: A Review on Growth and Functionality of $\mathrm{TiO}_{2}$ Nanotubes and Other Self-Aligned $\mathrm{MO}_{\mathrm{x}}$ Structures, Chem. Commun. 2009, 2791-2808.

112 Shinde, V.R.; Lokhande, C.D.; Mane, R.S.; Han S.H.; Hydrophobic and Textured ZnO Films Deposited by Chemical Bath Deposition: Annealing Effect, Appl. Surf. Sci. 2005, 245, 407-413.

113 Zhua, X.; Chena, J.; Scheidelera, L.; Reichlb, R.; Gerstorfer, J.G. Effects of Topography and Composition of Titanium Surface Oxides on Osteoblast Responses, Biomaterials, 2004, 25, 4087-4103.

${ }^{114}$ Yao, C.; Slamovich, E.; Webster, T. Enhanced Osteoblast Functions on Anodized Titanium With Nanotubue-Like Structures, J. Biomed. Mater. Res. 2007, 157-166.

115 Bjursten, L. M.; Rasmusson, L.; Oh, S.; Smith, G. C.; Brammer, K. S.; Jin, S. Titanium Dioxide Nanotubes Enhance Bone Bonding In Vivo, J. Biomed. Mater. Res. 2010, 92A 1218-1224.

${ }^{116}$ Hazan, R.; Sreekantan, S.; Khalil, A.A.; Nordin, I.M.S., Mat, I. ; Surface Engineering of Titania for Excellent Fibroblast 3T3 Cell-Metal Interaction. J. Phys. Sci., 2009, 20, 3547. 
${ }^{117}$ Bauer, S.; Park, J.; Mark, K. V.D.; Schmuki, P. Improved Attachment of Mesenchymal Stem Cells on Super-Hydrophobic TiO2 Nanotubes, Acta Biomater. 2008, 4 , 1576-1582.

118 Yao, C.; Slamovich, E.; Webster, T. Enhanced Osteoblast Functions on Anodized Titanium with Nanotubue-Like structures, J. Biomed. Mater. Res. 2007, 157-166.

${ }^{119}$ Park, J.; Bauer, S.; Mark, K.V.D.; Schmuki, P. Nanosize and Vitality: $\mathrm{TiO}_{2} \mathrm{Nanotube}$ Diameter Directs Cell Fate, Nano Lett. 2007, 7, 1686-1691.

${ }^{120}$ Oh, S.; Daraio, C.; Chen, L.-H., Pisanic, T. R.; Finones, R. R.; Jin, S. Significantly Accelerated Asteoblast Cell Growth on Aligned $\mathrm{TiO}_{2}$ Nanotubes, J. Biomed. Mater. Res. 2006, 78, 97-103.

${ }^{121}$ Brammer, K.; Oh, S.; Cobb, C. J.; Bjursten, L.M.; Heyde, H.V.D.; Jin, S.; Improved Bone-Forming Functionality on Diameter-Controlled $\mathrm{TiO}_{2}$ Nanotube Surface, Acta Biomaterialia, 2009, 5, 3215-3218.

122 Oh, S.; Brammer, K.S.; Li, Y.S. J.; Teng, D.; Engler, A.; Chien, S.; Jin, S. Stem Cell Fate Dictated Solely by Altered Nanotube Dimension, PNAS, 2009, 106, 2130-2135.

123 Popat, K. C.; Leoni, L.; Grimes, C.; Desai, T. A. Influence of Engineered Titania Nanotubular Surfaces on Bone Cells, Biomaterials, 2007, 28, 3188-3197.

${ }^{124}$ Wang, D.; Christensen, K.; Chawla, K.; Xiao, G.; Krebsbach, P. H.; Francesch, R. T. Isolation and Characterization of MC3T3-E1 Preosteoblast Subclones with Distict In Vitro and In Vivo Differentiation/Mineralization Potential. J. Bone Miner. Res., 1999, 14, 893-903.

125 Sasaki, K.; Asanuma, K.; Johkura, K.; Kasuga, T.; Okouchi, Y.; Ogiwara, N.; Kubota, S.; Teng, R.; Cui, L.; Zhao, X. Ultrastructural Analysis of TiO2 Nanotubes with Photodecomposition of Water into $\mathrm{O}_{2}$ and $\mathrm{H}_{2}$ Implanted in the Nude Mouse. Ann. Anat. 2006, 188, 137-142.

${ }^{126}$ Jang, J. M.; Park, S. J.; Choi, G. S.; Kwon T. Y.; Kim, K. H. Chemical State and UltraFine Structure Analysis of Biocompatible TiO2 Nanotube-Type Oxide Film Formed on Titanium Substrate. Met. Mater. Int. 2008, 14, 457-464.

${ }^{127}$ Garcia-Ripoll, A.; Amat, A. M.; Argues, A.; Vicente, R.; Martin, M. M. M; Sanchez Perez, J.A.; Oller I.; Malato, S. Confirming Pseudomonas Putida as a Reliable Bioassay for Demonstrating Biocompatibility Enhancement by Solar Photo Oxidative Processes of a Biorecalcitrant Effluent. J. Hazard. Mater. 2009, 162, 1223-1227.

${ }^{128}$ Lee, C.; Hong, C. Kim, H.; Kang, Jungwoo, Zheng, H. M. TiO2 Nanotubes as a Therapeutic Agent for Cancer Thermotherapy, Photochem. Photobio., 2010, 86, 981-989. 
129 Puleo, D.A.; Bizios, R. Formation of Focal Contacts by Osteoblasts Cultured on Orthopedic Biomaterials. J. Biomed. Mater. Res. 1992, 26, 291-301.

130 Sasaki, K.; Asanuma, K.; Johkura, K.; Kasuga, T.; Okouchi, Y.; Ogiwara, N.; Kubota, S.; Teng, R.; CuI, L.; Zhao, X. Ultrastructural Analysis of TiO2 Nanotubes with Photodecomposition of Water into $\mathrm{O} 2$ and $\mathrm{H} 2$ Implanted in the Nude Mouse. Ann. Anat. 2006, 188, 137-142.

131 Jang, J. M.; Park, S. J.; Choi, G. S.; Kwon T. Y.; Kim, K. H. Chemical State and Ultra-Fine Structure Analysis of Biocompatible TiO2 Nanotube-Type Oxide Film Formed on Titanium Substrate. Met. Mater. Int. 2008, 14, 457-464.

${ }^{132}$ Garcia-Ripoll, A.; Amat, A. M.; Argues, A.; Vicente, R.; Martin, M. M. B.; Perez, I. Oller, J. A. S.; Malato, S. Confirming Pseudomonas Putida as a Reliable Bioassay for Demonstrating Biocompatibility Enhancement by Solar Photo Oxidative Processes of a Biorecalcitrant Effluent. J. Hazard. Mater. 2009, 162, 1223-1227.

${ }^{133}$ Lee, C.; Hong, C.; Kim, H.; Kang, J.; Zheng, H.M. TiO 2 Nanotubes as a Therapeutic Agent for Cancer Thermotherapy, Photochem. Photobio., 2010, 86, 981-989.

134 S. Oh, C. Daraio, L.-H. Chen, T. R. Pisanic, R. R. Finones, S. Jin, Significantly accelerated osteoblast cell growth on aligned $\mathrm{TiO}_{2}$ nanotubes, J. Biomed. Mater. Res. 2006, 78, 97-103.

${ }^{135}$ Variola, F.; Vetrone, F.; Richert, L.; Jedrzejowski, P.; Yi, J.-H.; Zalzal, S.; Clair, S.; Sarkissian, A.; Perepichka, D.F.; Wuest, J.D.; Rosei, F.; Nanci, A.; Improving Biocompatibility of Implantable Metals by Nanoscale Modification of Surfaces: An Overview of Strategies, Fabrication Methods, and Challenges, Small, 2009, 5, 996-1006.

${ }^{136}$ Park, J.-W.; Kim, Y.-J.; Jang, J.-H.; Enhanced Osteoblast Response to Hydrophilic Strontium and/or Phosphate Ions-Incorporated Titanium Oxide Surfaces, Clin. Oral Impl. Res., 21 (2010), 398-408.

137 Eisenbarth, E.; Velten, D.; Muller, M.; Thull, R. Breme, J. Biocompatibility of Beta Stabilizing Elements of Titanium Alloys, Biomaterials, 2004, 25, 5705-5713.

138 Wapner, K.L. Implications of Matallic Corrosion in Total Knee Arthoroplasty. Clin Orthop. 1991, 271, 12-20.

${ }^{139}$ Guo, W.-H.; Frey, M. T.; Burnham, N.A.; Wang Y.-L. Substrate Rigidity Regulates the Formation and Maintenance of Tissues, Biophys. J. 2006, 90, 2213-2220.

${ }^{140}$ Lenhert, S.; Meier, M.B.; Meyer, U.; Chi, L.; Wiesmann, H.P. Osteoblast Alignment, 
Elongation and Migration on Grooved Polystyrene Surfaces Patterned by Langmuire Blodgett Lithography. Biomaterials 2005; 26, 563-571.

141 Meyle, J.; Wolburg, H.; Recum, AF. Surface Micromorphology and Cellular Interactions. J. Biomater. Appl. 1993, 7(4):362-74.

${ }^{142}$ Charest, JL.; Bryant, LE.; Garcia, AJ.; King, W.P. Hot Embossing for Micropatterned Cell Substrates. Biomaterials ,2004, 25, 4767-4775.

143 Matsuzaka, K.; Walboomers, XF.; Yoshinari, M.; Inoue, T.; Jansen, J.A.; The Attachment and Growth Behavior of Osteoblast-Like Cells on Microtextured Surfaces, Biomaterials, 2003, 24, 2711-2719.

144 Leser, V.; Drobne, D.; Pipan, Z.; Milani, M.; Tatti, F. Comparison of Different Preparation Methods of Biological Samples for FIB Milling and SEM Investigation, $J$. Microscopy, 2009, 233, 309-319.

145 Popat, K. C.; Leoni, L.; Grimes, C.; Desai, T. A. Influence of Engineered Titania Nanotubular Surfaces on Bone Cells, Biomaterials, 2007, 28 , 3188-3197.

146 Oh, S.; Daraio, C.; Chen, L.-H.; Pisanic, T. R.; Finones, R. R.; Jin, S. Significantly Accelerated Osteoblast Cell Growth on Aligned $\mathrm{TiO}_{2}$ Nanotubes, J. Biomed. Mater. Res. 2006, 78, 97-103.

147 Gere, J. M.; Timoshenko, S. P. Mechanics of Materials, $2^{\text {nd }}$ ed. Brooks/Cole, Monterey, CA, 1984, 7, 351.

148 Shokuhfar, T.; Arumugam, G. K.; Heiden P. A.; Yassar, R. S.; Friedrich, C. Direct Compressive Measurements of Individual Titanium Dioxide Nanotubes, ACS Nano, 2009, 3, 3098.

${ }^{149}$ Khatiwala, C.B.; Peyton, S. R.; Putnam, A.J. Osteogenic Differentiation of MC3T3E1 Cells Regulated by Substrate Stiffness Requires Mapk Activation, The 2006 AIchE Annual Meeting, Nov. 11-14, 2006, San Francisco, CA.

150 Khatiwala, C. B., Peyton, S. R.; Putnam, A. J. The Intrinsic Mechanical Properties of the Extracellular Matrix Affect the Behavior of Pre-Osteoblastic MC3T3-E1 Cells. Am J Physiol. Cell Physiol. 2006, 290, 1640-1650

${ }^{151}$ Peyton, S.R.; Putnam, A.J.; Extracellular Matrix Rigidity Governs Smooth Muscle Cell Motility in a Biphasic Fashion, J. Cell Physiol. 2005, 204,198-209. 
152 Wang, H.B.; Dembo, M.; Wang, Y.L.; Substrate Flexibility Regulates Growth and Apoptosis of Normal but not Transformed Cells, Am. J. Physiol. Cell Physiol. 2000, 279, 1345-1350.

${ }^{153}$ Engler, A.J.; Griffin, M.A.; Sen, S.; Bonnemann, C.G.; Sweeney, H.L.; Discher, D.E.; Myotubes Differentiate Optimally on ubstrates with Tissue-Like Stiffness: Pathological implications for soft or stiff microenvironments, J. Cell Biol. 2004, 166, 877-87.

154 Robert, J.; Pelham, Jr.; Wang, Y.-1. Cell Locomotion and Focal Adhesions Are Regulated by Substrate Flexibility, Proc. Natl. Acad. Sci. 1997, 94,13661-13665.

155 Palecek, S.P, Loftus, J.C.; Ginsberg, M.H.; Lauffenburger, D.A.; Horowitz, A. F. Nature, 1997, 385,537-540.

156 Bernstein, L.R.; Liotta, L.A. Molecular Mediators of Interactions with Extracellular Matrix Components in Metastasis and Angiogenesis, Curr Opin Oncol , 1994, 6 ,106113 ,

${ }^{157}$ Lo, C.-M.; Wang, H.-B.; Dembo, M. Wang, Y.-L. Cell Movement is Guided by the Rigidity of the Substrates. Biophys. 2000, 79,144-152.

158 Foty, R. A.; Pfleger, C. M.; Forgacs, G.; Steinberg, M. S. Surface Tensions of Embryonic Cells Predict Their Mutual Envelopment Behavior, Development 1996, 122, 1611-1620.

${ }^{159}$ Bernstein, L.R., Liotta, L. A. Molecular Mediators of Interactions with Extracellular Matrix Components in Metastasis and Angiogenesis, Curr. Opin. Oncol. 1994, 6, 106113.

160 Khatiwala, C. B.; Peyton, S. R.; Putnam, A. J. The Intrinsic Mechanical Properties of the Extracellular Matrix Affect the Behavior of Pre-Osteoblastic MC3T3-E1 Cells. Am. J. Physiol. Cell. Physiol. 2006, 290, C1640-50

${ }^{161}$ Guo, W.; Margo, T.; Frey, N.; Burnham, A.;Wang, Y.; Substrate Rigidity Regulates the Formation and Maintenance of Tissues, Biophys J. 2006, 90, 2213-2220.

${ }^{162}$ Wang, H.-B.; Dembo, M.; Wang, Y.-L. Substrate Flexibility Regulates Growth and Apoptosis of Normal but Not Transformed Cells, Am. J. Physiol. Cell Physiol. 2000, 279, 1345-1350.

163 Chen, C. S.; Mrksich, M.; Huang, S.; Whitesides, G.M.; Ingber, D. E., Geometric Control of Cell Life and Death, Science, 1997, 276, 1425-1428. 
164 Guo, W.; Frey, M.T.; Burnham, N.A., Wang, Y. Substrate Rigidity Regulates the Formation and Maintenance of Tissues, Biophys J. 2006 , 90, 2213-2220.

165 Ercan, B.; Webster, T.J. The Effect of Biphasic Electrical Stimulation on Osteoblast Function at Anodized Nanotubular Titanium Surfaces, Biomaterials, 2010, 31, 36843693.

166 Kalbacova, M.; Macak, J. M.; S.-Stein, F.; Mierke, C. T.; Schmuki, P. TiO2 Nanotubes: Photocatalyst for Cancer Cell Killing, Phys. Stat. Sol. 2008, 4, 194-196.

${ }^{167}$ Popata, K. C.; Eltgrothc, M.; LaTempad, T. J.; Grimesd, C. A.; Desaia, T. A. Decreased Staphylococcus Epidermis Adhesion and Increased Osteoblast Functionality on Antibiotic-Loaded Titania Nanotubes, Biomaterials 2007, 28, 4880-4888.

${ }^{168}$ Lee, C.; Hong, C.; Kim, H., Kang, J., Zheng, H.M. TiO 2 Nanotubes as a Therapeutic Agent for Cancer Thermotherapy, Photochemistry and Photobiology 2010, 86: 981-989. 